\title{
The 2017 National Nursing Workforce Survey
}

\author{
Richard A. Smiley, MS, MA \\ Statistician, National Council of State Boards of Nursing \\ Pamela Lauer, MPH \\ Program Director, Texas Center for Nursing Workforce Studies \\ Research Committee Chair, The National Forum of State Nursing Workforce Centers
}

\section{Cynthia Bienemy, PhD, RN}

Director, Louisiana State Board of Nursing-Center for Nursing

President, The National Forum of State Nursing Workforce Centers

Judith G. Berg, MS, RN, FACHE

Chief Executive Officer, HealthImpact

Past President, The National Forum of State Nursing Workforce Centers

Emilie Shireman, PhD

Data Scientist, National Council of State Boards of Nursing

Kyrani A. Reneau, MA

Research Coordinator, National Council of State Boards of Nursing

\section{Maryann Alexander, PhD, RN, FAAN}

Chief Officer, Nursing Regulation, National Council of State Boards of Nursing 


\section{CONTENTS}

OCtober 20 i 8 • Volume 9 - Issue 3 Supplement

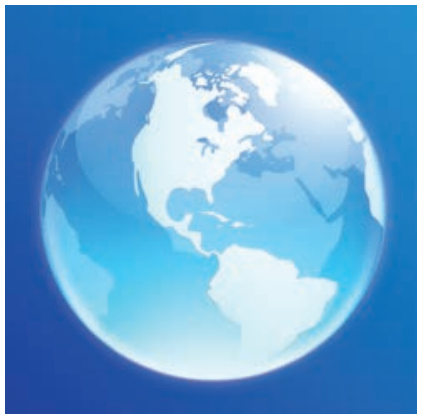

Advancing nursing excellence for public protection

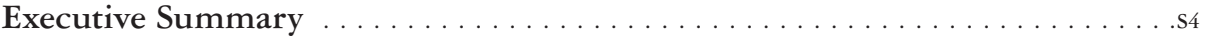

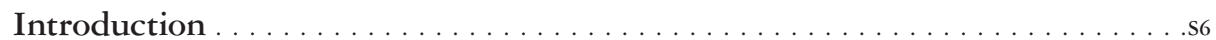

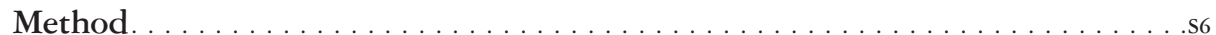

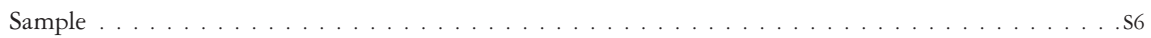

Survey Instrument and Materials . . . . . . . . . . . . . . . . . . . . . . . . . s9

Procedure. . . . . . . . . . . . . . . . . . . . . . . . . . . . . . . . . . .

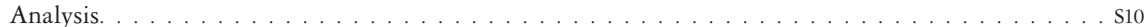

Population Estimate . . . . . . . . . . . . . . . . . . . . . . . . . . . s10

Registered Nurse Results . . . . . . . . . . . . . . . . . . . . s11

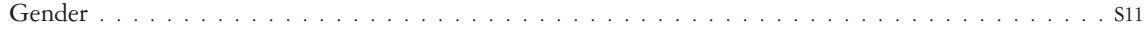

Race/Ethnicity . . . . . . . . . . . . . . . . . . . . . . . . . . . . . . .

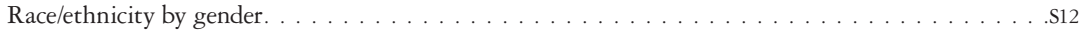

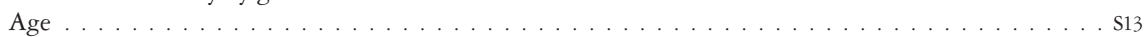

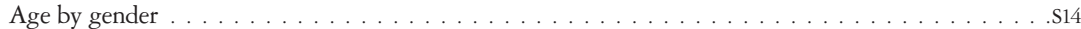

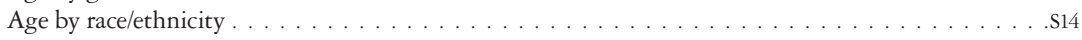

Education

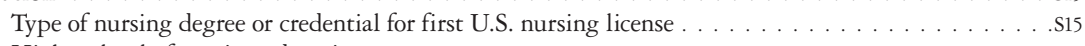

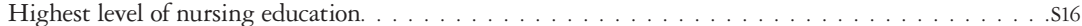

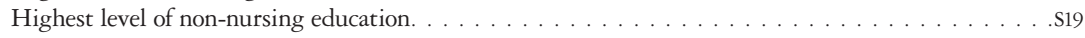

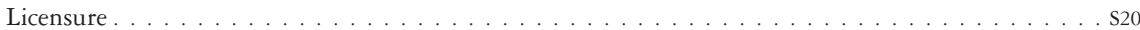

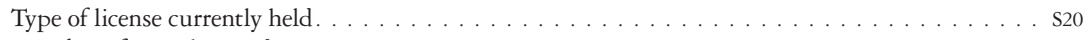

Number of years licensed . . . . . . . . . . . . . . . . . . . . . . . . . . . . . . . . . . . . . .

Initially licensed in the United States. . . . . . . . . . . . . . . . . . . . . S22

Credentialed to practice as an APRN . . . . . . . . . . . . . . . . . . . . . . . . . . .

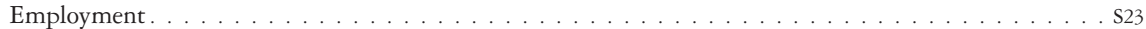

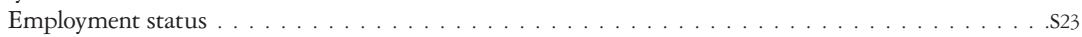

Reasons for being unemployed . . . . . . . . . . . . . . . . . . . . . . . . . . . . S24

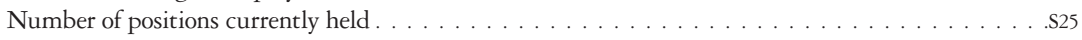

Number of hours worked during a typical week in all nursing positions. . . . . . . . . . . . . . S26

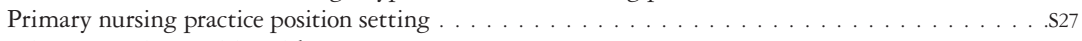

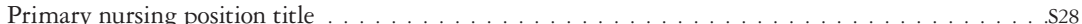

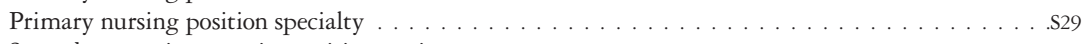

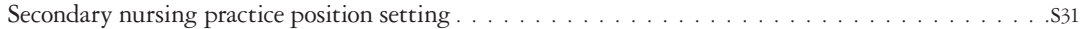

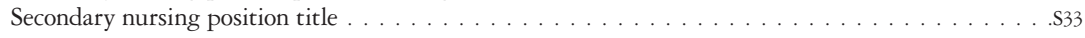

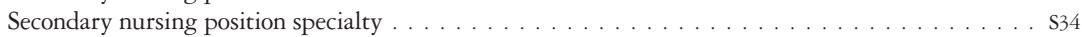

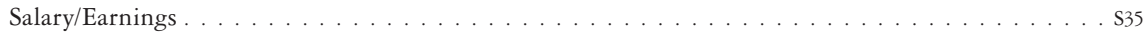

2016 pre-tax annual earnings from primary nursing position. . . . . . . . . . . . . . . S35

Earnings by gender and specialty . . . . . . . . . . . . . . . . . . . . . . . . . . . . . . . . . .

Earnings by highest education $\ldots \ldots \ldots \ldots \ldots \ldots \ldots \ldots \ldots \ldots \ldots \ldots \ldots \ldots \ldots \ldots \ldots \ldots$

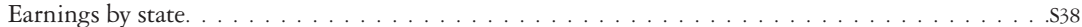

Earnings by years licensed and age . . . . . . . . . . . . . . . . . . . . . . . . . .

Earnings by APRNs . . . . . . . . . . . . . . . . . . . . . . . . . . . S40

Telehealth Utilization . . . . . . . . . . . . . . . . . . . . . 440

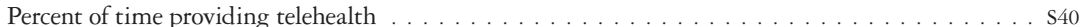

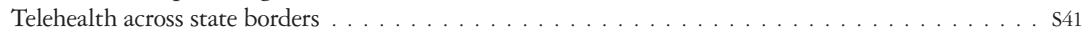

Telehealth across national borders . . . . . . . . . . . . . . . . . . . . . . . S42

Modes of communication used for telehealth . . . . . . . . . . . . . . . . . \$42

Discussion and Implications . . . . . . . . . . . . . . . . . . . . . . . . . . . . s43

Limitations . . . . . . . . . . . . . . . . . . . . . . . . . . . S45

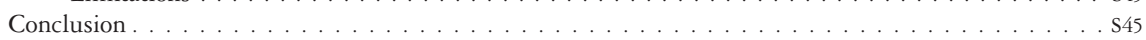

Licensed Practical Nurse/Licensed Vocational Nurse Results . . . . . . . . . . . s46

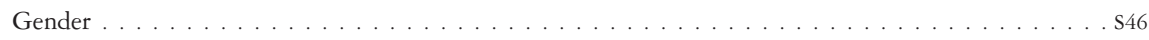

Race/Ethnicity . . . . . . . . . . . . . . . . . . . . . . . . . . \$ \$46

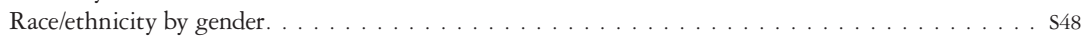

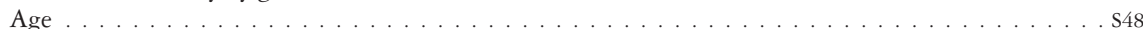

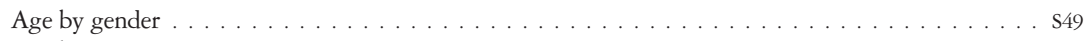

Age by race/ethnicity . . . . . . . . . . . . . . . . . . . . . . . . . . . . . . . . .

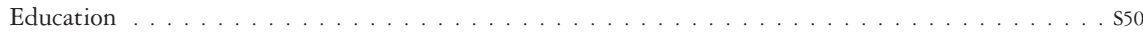

Type of nursing degree or credential for first U.S. nursing license . . . . . . . . . . . s50

Highest level of nursing education. . . . . . . . . . . . . . . . . . . . . . . . . . . . . . .

Highest level of non-nursing education. . . . . . . . . . . . . . . . . . . . . . . . . . .

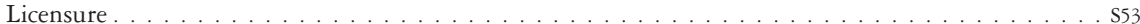

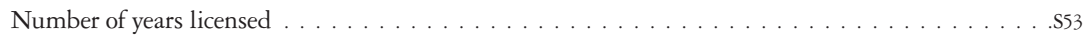

Initially licensed in the United States. . . . . . . . . . . . . . . . . . . S54 


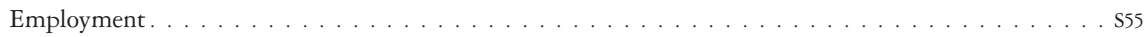

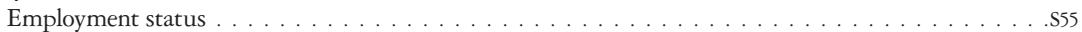

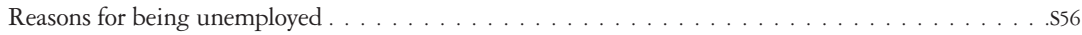

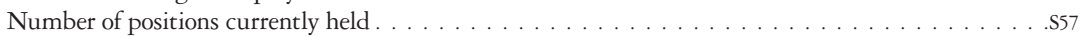

Number of hours worked during a typical week in all nursing positions . . . . . . . . . . . S57

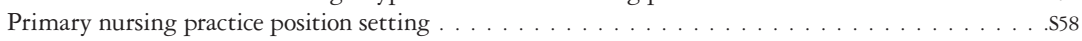

Primary nursing position title ........... S60

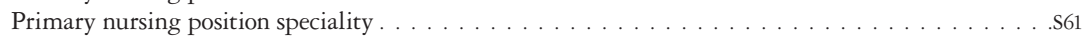

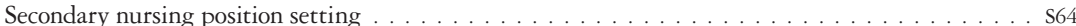

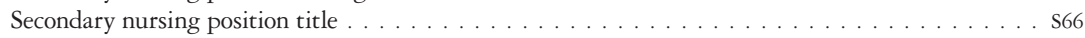

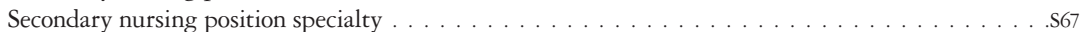

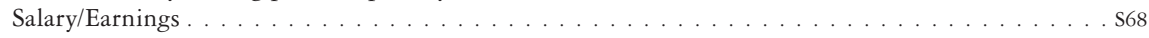

2016 pre-tax annual earnings from primary nursing position. . . . . . . . . . . . . . . S68

Earnings by gender and specialty . . . . . . . . . . . . . . . . . . . . . S69

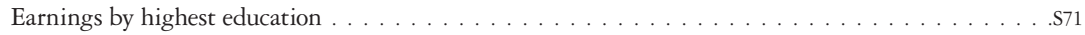

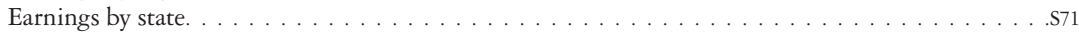

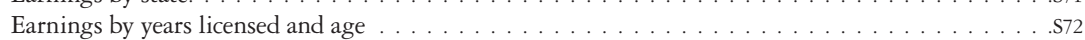

Telehealth Utilization . . . . . . . . . . . . . . . . . . . . . . . . . . . . . . S72

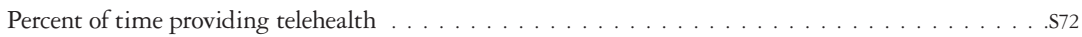

Telehealth across state borders . . . . . . . . . . . . . . . . . . . . . . . . . . . . . . S73

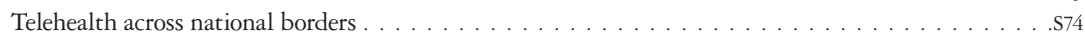

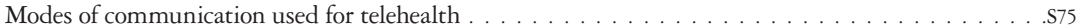

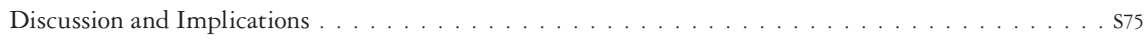

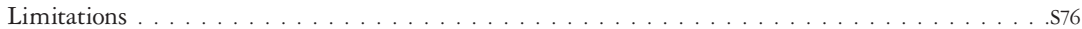

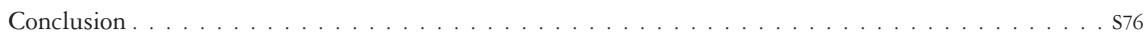

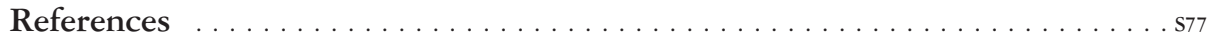

Appendices . . . . . . . . . . . . . . . . . . . . . . . . . . . . . . . . . . .

Appendix A: Registered Nurse Nonresponse Analysis and Sample Weighting . . . . . . . . . . . . . . . S78 Appendix B: Licensed Practical Nurse/Licensed Vocational Nurse Nonresponse Analysis and Sample

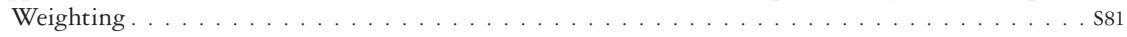

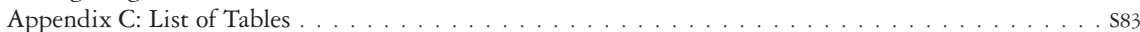

Appendix D: 2017 National Workforce Study Questionnaire . . . . . . . . . . . . . . . . . . S85 


\section{The 2017 National Nursing Workforce Survey}

\section{Executive Summary}

The purpose of this study is to provide the nation with the most accurate data available on the characteristics of the U.S. nursing workforce. Since 2013, the National Council of State Boards of Nursing (NCSBN) and The National Forum of State Nursing Workforce Centers (Forum) have partnered every 2 years to conduct this national sample survey using the Forum's Nurse Supply Minimum Data Set (MDS), a standardized survey tool designed to collect workforce data. These data will help answer some of the pressing questions asked by health care workforce researchers, policy makers, and leaders in nursing education and practice to assist in workforce planning and provide evidence for decisions impacting the future of nursing in America.

Previously, NCSBN and the Forum reported on the state of the workforce in The 2015 National Nursing Workforce Survey. The 2015 study estimated that in the United States and its territories, 3,852,881 individuals held active registered nurse (RN) licenses, and 906,471 held an active licensed practical nurse/licensed vocational nurse (LPN/LVN) license (Budden, Moulton, Harper, Brunell, \& Smiley, 2016). Approximately $12 \%$ of these nurses held multiple licenses. The study identified an advanced practice registered nurse (APRN) workforce that appeared to be increasing in age in addition to an aging population of nurse educators. Eighty-one percent of respondents with RN licenses were actively employed in nursing and $77 \%$ of those with LPN/ LVN licenses were actively employed in nursing. According to the 2015 study, hospitals were the most common employment setting for RNs (or $54 \%$ of respondents). However, the authors recognized that the work setting for both RNs and LPNs/LVNs was changing due to telehealth technologies and other factors. Demographically, the 2015 survey found that $19.5 \%$ of RN respondents and $32 \%$ of LPN/LVN respondents were from underrepresented racial and ethnic populations. The study also noted that minority populations were better represented in younger cohorts and among more recently licensed RNs. Data from this survey indicated that the median salary for males was higher than for females among both RNs and LPNs/LVNs $(\$ 72,000$ versus $\$ 64,000$ among $R N$ s, and $\$ 43,200$ versus $\$ 38,000$ among LPNs/LVNs.

\section{Selected Findings From the 2017 Workforce Study}

\section{Size of the RN and LPN/LVN Workforce}

As of December 2017, the total number of active RN licenses held was 4,639,548 and active LPN/LVN licenses held was 975,988 , representing an increase by 261,275 of RN licenses $(6.0 \%)$ and a decrease by 52,432 of LPN/LVN licenses (-5.1\%) when compared to 2015. After adjusting for nurses with multiple licenses, the 2017 numbers correspond to approximately 3,951,001 RN licensees and 920,743 LPN/LVN licensees.

\section{Aging of the Workforce}

The average age of RNs is 51, which is largely consistent with previous study findings (2015 and 2013). In 2015, 12.4\% of the RN respondents were 65 or older compared to $14.6 \%$ in the current study, an increase of 2.2 percentage points, which indicates a slow but steady growth of RNs that may be heading for retirement. The average age of LPNs/LVNs was 52 in 2017 , a year older than in 2015. The percentage of nurses 65 and older increased from $9.9 \%$ in 2015 to $13.2 \%$ in 2017 , which represents twice as much of an increase in comparison to RNs.

\section{Gender, Race, and Ethnic Diversity}

The percentage of male RNs (9.1\%) in the 2017 workforce increased by 1.1 and 2.5 percentage points, respectively, when compared with findings from the 2015 (8.0\%) and 2013 study (6.6\%). Approximately $5 \%$ of RNs indicated that they were of Hispanic or Latino origin. In the current study $19.2 \%$ of the RN respondents were minorities, which includes other and two or more races: Asian (7.5\%), Black/African American (6.2\%), other (2.9\%), and two or more races (1.7\%). The percentage of nurses who identify as Asian in the current study exceeds the 5.7\% represented in the U.S. population; the percentage of nurses who identify as Black/African American falls below the $13.3 \%$ in the U.S. population (U.S. Census Bureau, 2017). The LPN/LVN workforce remains relatively unchanged in terms of gender diversity between 2015 and 2017. The percentage of males in the LPN/LVN workforce increased by less than half of a percentage point from $7.5 \%$ to $7.7 \%$ between the two studies. LPNs/LVNs are more racially and ethnically diverse than their RN counterparts. Approximately $29 \%$ of LPNs/LVNs were racial minorities with the largest reporting minority group being Black/African Americans (18.5\%). 


\section{Education}

The trend toward a higher percentage of respondents pursuing a BSN degree as their initial nursing education continued in 2017 with $41.8 \%$ of the RNs reporting the BSN as the degree that qualified them for their first US nursing license, which reflects a 2.8 percentage point increase when compared to $2015(39.0 \%)$ and a 6.3 percentage point increase when compared to $2013(35.5 \%)$. In terms of graduate education, the number of RNs with a Master's degree in nursing has increased from $13.8 \%$ in 2013 to $17.1 \%$ in 2017, an increase of 3.3 percentage points. Although small in terms of percentage, $1.1 \%$ of responding RNs reported having a Doctorate in Nursing Practice (DNP) as their highest level of nursing education, which is double the percent of nurses who reported having a DNP in 2015. By comparison, 83.2\% of LPNs/LVNs indicated that their entry-level qualification for their first US nursing license was a vocational/practical certificate. Of those, more than three-quarters did not pursue a higher level of nursing education.

\section{Employment and Salary}

Nurses work settings are changing, as patient care is no longer confined to the walls of a health care facility. Hospitals were the primary employment setting for $55.7 \%$ of RNs, $54.4 \%$ in 2015 , but less than the $56.5 \%$ reported in 2013 . Ambulatory care setting was the second most frequently selected employment setting reported by $9.4 \%$ of RNs, followed by nursing home/ extended care $(4.8 \%)$, and home health $(4.3 \%)$.

The median pre-tax annual earnings for RNs in the current study increased from $\$ 60,000$ in 2015 to $\$ 63,000$ in 2017, which is lower than the median salary of $\$ 70,000$ for RNs reported by the Bureau of Labor Statistics Occupational Outlook Handbook (2018). In the current study, responding RNs making between $\$ 80,000$ and $\$ 100,000$ increased by 1.6 percentage points and those making more than $\$ 100,000$ increased by nearly 4 percentage points.

Nursing home/extended care was the most common work setting for LPNs/LVNs (31.7\%), followed by home health settings $(14.0 \%)$, and hospitals $(9.6 \%)$. These findings are consistent with results from the 2015 survey.

The median pre-tax annual earnings for LPNs/LVNs increased from $\$ 38,000$ in 2015 to $\$ 40,000$ in 2017. However, it is also lower than the median pay of $\$ 45,030$ reported by the Bureau of Labor Statistics (2018).

\section{Telehealth}

Over half (54.1\%) of responding RNs provided nurse services using telehealth technologies. In the current study, $45.7 \%$ provided these services across a state border compared to $39.4 \%$ in 2015 , an increase of 6.3 percentage points, and $11.1 \%$ reported providing remote services to patients or clients across national borders, which is an increase of 3.4 percentage points when compared to 2015 (7.7\%).

LPNs/LVNs provided nursing services using telehealth technologies in similar proportions to RNs (54.2\%). Of those providing these services, $43.9 \%$ provided services across state borders, an increase of 10.1 percentage points from 2015.

\section{Conclusion}

In conclusion, ongoing monitoring and evaluation of the nursing workforce are critical, as the nursing workforce will continue to undergo significant changes in the next few years. This survey represents the nursing workforce of today with the expectation that the workforce of tomorrow will be slightly younger, highly educated, with higher numbers working in the community providing primary health care and using technology and telehealth as a means to deliver health care. As the United States undergoes health system transformations, nurses, as the largest segment of the health care workforce and members of the interprofessional health care team, are in a unique position to contribute to and, in many instances, lead the transformation. This effort will require a nursing workforce with an enhanced set of knowledge and skills in relation to population health and wellness, multidisciplinary care coordination, technological advances in health care delivery, evidence-based data analytics, and quality improvement. All these elements create a picture of what will constitute the nursing workforce of the future. 


\section{Introduction}

The National Council of State Boards of Nursing (NCSBN) and The Forum of State Nursing Workforce Centers (Forum) collaborated on the third biennial national survey of registered nurses (RNs) and licensed practical nurses/licensed vocational nurses (LPNs/

LVNs) to provide an in-depth study of the national nursing workforce. The survey is designed to collect important information on the supply of RNs and LPNs/LVNs in the United States. Knowledge of the supply of nurses can be used to predict potential shortages and assist in the allocation of resources, program development, and recruitment efforts in both the health care system and education sectors. Having an adequate supply of nurses in the workforce is essential to providing safe and effective health care to the citizens of the United States.

This study presents a national, randomized survey of 148,684 licensed RNs and 151,928 LPNs/LVNs. Data were collected between August 28, 2017, and January 15, 2018, from the 48,704 RN responders (32.8\%) and the 40,272 LPN/LVN responders (26.5\%). The data provides the most recent and detailed information on the current demographics, characteristics, educational attainment, employment, and trends of the U.S. RN and LPN/LVN nursing workforce as of 2017. To illustrate trends in workforce supply, the current study's results were compared to some national nursing workforce studies that based their findings on data collected years prior to this study, as well as studies in which different data collection methods were used. Thus, the comparisons of statistics from various studies presented should be interpreted with the above considerations in mind.

\section{Method}

\section{Sample}

All RNs and LPNs/LVNs with an active license in the United States and its territories were eligible candidates for survey participation. A portion of the sample was drawn from Nursys ${ }^{\circledR}$, NCSBN’s licensure database. This database contains basic demographic and licensure information for RN and LPN/LVN licensees, with the exception of Hawaii, Louisiana (LPN), and Oklahoma licensees because at the time of the study, these states did not participate in Nursys. Additionally, Alabama, Kansas, Massachusetts, Pennsylvania, and Washington restrict the use of addresses in Nursys. These states were contacted to obtain licensee lists and addresses directly. Licensee lists and addresses were also obtained directly from the following BONs: California (RN and LPN/LVN), Michigan, New Jersey, and New York. Names and addresses of nurses living in Utah were obtained from a national mailing list. American Samoa and Missouri voluntarily chose not to participate in this survey.

As of June 2017, the total number of active RN licenses held was 4,639,548 and active LPN/LVN licenses held was 975,988. These numbers do not include American Samoa and Missouri but do include the approximately $17 \%$ of individuals with multiple licenses. Separate RN and LPN/LVN samples stratified by state were randomly selected from the RN licensees and LPN/LVN licensees. Surveys were mailed to 153,920 RNs and 158,502 LPNs/LVNs. Table 1 and Table 2 present the sampling by jurisdiction/state. Each jurisdiction is listed with the actual number of active licenses at the time of sampling and the number of surveys that needed to be received from each jurisdiction in order to construct a $95 \%$ confidence of plus or minus $3 \%$ error. Regardless of jurisdiction size, this calculated out to be approximately 1,000 nurses per jurisdiction. To calculate the number of surveys needed to be mailed out to reach the target survey response, response rates via online and paper from the previous 2015 survey administration were used as estimates. For example, for Alaska in 2015 there was a 36\% response rate for the RN survey. Given this estimated rate, 2,684 RNs in Alaska were selected to be sent a survey in order to receive the target of 977 surveys. Actual response from Alaska RNs to the current survey (i.e., the number of responses returned) was 966, very close to the target of 977.

\section{TABLE 1}

\section{Number of Active RN Licenses}

\begin{tabular}{lcccccccc}
\hline Jurisdiction & $\begin{array}{c}\text { Number of } \\
\text { Active RN } \\
\text { Licenses }\end{array}$ & $\begin{array}{c}\text { Estimated } \\
\text { Sample: 95\% } \\
\text { Confidence, } \\
\text { 3\% Error }\end{array}$ & $\begin{array}{c}\text { Estimated } \\
\text { Total } \\
\text { Response } \\
\text { Rate }\end{array}$ & $\begin{array}{c}\text { Number of } \\
\text { Surveys } \\
\text { Mailed }\end{array}$ & Undeliverable & $\begin{array}{c}\text { Number } \\
\text { Received }\end{array}$ & $\begin{array}{c}\text { Number of } \\
\text { Responses }\end{array}$ & $\begin{array}{c}\text { Total } \\
\text { Response } \\
\text { Rate }\end{array}$ \\
\hline AK & 13,829 & 977 & $36.4 \%$ & 2,684 & 49 & 2,635 & 966 & $36.7 \%$ \\
\hline AL & 79,610 & 1,051 & $28.1 \%$ & 3,740 & 30 & 3,710 & 1,010 \\
\hline AR & 41,636 & 1,040 & $30.1 \%$ & 3,455 & 67 & 3,388 & 967 & $27.2 \%$ \\
\hline CA & 87,420 & 1,054 & $32.8 \%$ & 3,213 & 231 & 2,982 & 966 \\
\hline CO & 427,892 & 1,064 & $32.1 \%$ & 3,315 & 74 & 3,241 & 1,020 \\
\hline
\end{tabular}




\begin{tabular}{|c|c|c|c|c|c|c|c|c|}
\hline Jurisdiction & $\begin{array}{l}\text { Number of } \\
\text { Active RN } \\
\text { Licenses }\end{array}$ & $\begin{array}{r}\text { Estimated } \\
\text { Sample: } 95 \% \\
\text { Confidence, } \\
3 \% \text { Error } \\
\end{array}$ & $\begin{array}{c}\text { Estimated } \\
\text { Total } \\
\text { Response } \\
\text { Rate }\end{array}$ & $\begin{array}{l}\text { Number of } \\
\text { Surveys } \\
\text { Mailed }\end{array}$ & Undeliverable & $\begin{array}{l}\text { Number } \\
\text { Received }\end{array}$ & $\begin{array}{l}\text { Number of } \\
\text { Responses }\end{array}$ & $\begin{array}{c}\text { Total } \\
\text { Response } \\
\text { Rate }\end{array}$ \\
\hline CT & 64,882 & 1,050 & $35.9 \%$ & 2,925 & 76 & 2,849 & 937 & $32.9 \%$ \\
\hline $\mathrm{DC}$ & 26,508 & 1,020 & $29.0 \%$ & 3,517 & 126 & 3,391 & 960 & $28.3 \%$ \\
\hline$\overline{D E}$ & 18,111 & 1,010 & $37.7 \%$ & 2,679 & 106 & 2,573 & 818 & $31.8 \%$ \\
\hline $\mathrm{FL}$ & 316,640 & 1,063 & $27.3 \%$ & 3,894 & 89 & 3,805 & 993 & $26.1 \%$ \\
\hline GA & 132,949 & 1,058 & $30.0 \%$ & 3,527 & 111 & 3,416 & 1,018 & $29.8 \%$ \\
\hline $\mathrm{HI}$ & 27,916 & 1,048 & $36.6 \%$ & 2,863 & 173 & 2,690 & 882 & $32.8 \%$ \\
\hline IA & 54,415 & 1,046 & $39.4 \%$ & 2,655 & 70 & 2,585 & 992 & $38.4 \%$ \\
\hline ID & 21,362 & 1,017 & $41.4 \%$ & 2,457 & 113 & 2,344 & 929 & $39.6 \%$ \\
\hline $\mathrm{IL}$ & 195,399 & 1,061 & $31.2 \%$ & 3,401 & 88 & 3,313 & 1,069 & $32.3 \%$ \\
\hline IN & 111,129 & 1,057 & $37.7 \%$ & 2,804 & 109 & 2,695 & 902 & $33.5 \%$ \\
\hline KS & 57,969 & 1,047 & $36.0 \%$ & 2,908 & 60 & 2,848 & 972 & $34.1 \%$ \\
\hline KY & 69,753 & 1,051 & $30.8 \%$ & 3,412 & 34 & 3,378 & 1,002 & $29.7 \%$ \\
\hline LA & 65,914 & 1,050 & $28.4 \%$ & 3,697 & 59 & 3,638 & 1,096 & $30.1 \%$ \\
\hline MA & 134,405 & 1,058 & $34.0 \%$ & 3,112 & 58 & 3,054 & 983 & $32.2 \%$ \\
\hline$M D$ & 81,363 & 1,053 & $31.4 \%$ & 3,354 & 114 & 3,240 & 1,080 & $33.3 \%$ \\
\hline $\mathrm{ME}$ & 25,026 & 1,022 & $36.2 \%$ & 2,823 & 60 & 2,763 & 1,066 & $38.6 \%$ \\
\hline $\mathrm{Ml}$ & 149,864 & 1,060 & $39.5 \%$ & 2,684 & 57 & 2,627 & 1,027 & $39.1 \%$ \\
\hline $\mathrm{MN}$ & 109,456 & 1,056 & $42.3 \%$ & 2,496 & 51 & 2,445 & 978 & $40.0 \%$ \\
\hline MS & 48,907 & 1,043 & $25.2 \%$ & 4,139 & 91 & 4,048 & 1,073 & $26.5 \%$ \\
\hline MT & 16,285 & 1,003 & $44.3 \%$ & 2,264 & 127 & 2,137 & 989 & $46.3 \%$ \\
\hline NC & 134,738 & 1,058 & $36.3 \%$ & 2,915 & 92 & 2,823 & 908 & $32.2 \%$ \\
\hline ND & 14,039 & 991 & $41.8 \%$ & 2,371 & 71 & 2,300 & 1,005 & $43.7 \%$ \\
\hline $\mathrm{NE}$ & 29,930 & 1,027 & $42.5 \%$ & 2,416 & 258 & 2,158 & 792 & $36.7 \%$ \\
\hline $\mathrm{NH}$ & 22,777 & 1,018 & $37.5 \%$ & 2,715 & 95 & 2,620 & 928 & $35.4 \%$ \\
\hline $\mathrm{NJ}$ & 124,991 & 1,058 & $30.5 \%$ & 3,469 & 47 & 3,422 & 1,092 & $31.9 \%$ \\
\hline NM & 28,422 & 1,027 & $33.1 \%$ & 3,103 & 142 & 2,961 & 1,035 & $35.0 \%$ \\
\hline NV & 38,054 & 1,036 & $30.9 \%$ & 3,353 & 165 & 3,188 & 1,016 & $31.9 \%$ \\
\hline $\mathrm{NY}$ & 322,755 & 1,063 & $35.4 \%$ & 3,003 & 294 & 2,709 & 707 & $26.1 \%$ \\
\hline $\mathrm{OH}$ & 204,281 & 1,062 & $37.1 \%$ & 2,863 & 102 & 2,761 & 895 & $32.4 \%$ \\
\hline$\overline{O K}$ & 55,506 & 1,046 & $30.5 \%$ & 3,430 & 69 & 3,361 & 935 & $27.8 \%$ \\
\hline OR & 60,230 & 1,048 & $37.4 \%$ & 2,802 & 104 & 2,698 & 1,075 & $39.8 \%$ \\
\hline PA & 220,583 & 1,062 & $39.8 \%$ & 2,668 & 38 & 2,630 & 937 & $35.6 \%$ \\
\hline $\mathrm{RI}$ & 20,529 & 1,013 & $33.9 \%$ & 2,988 & 113 & 2,875 & 968 & $33.7 \%$ \\
\hline SC & 69,799 & 1,050 & $32.2 \%$ & 3,261 & 95 & 3,166 & 940 & $29.7 \%$ \\
\hline SD & 18,162 & 1,006 & $42.4 \%$ & 2,373 & 50 & 2,323 & 940 & $40.5 \%$ \\
\hline TN & 100,817 & 1,056 & $31.7 \%$ & 3,331 & 94 & 3,237 & 891 & $27.5 \%$ \\
\hline$T X$ & 314,920 & 1,063 & $27.6 \%$ & 3,851 & 131 & 3,720 & 906 & $24.4 \%$ \\
\hline UT & 33,309 & 1,033 & $35.3 \%$ & 2,926 & 0 & 2,926 & 736 & $25.2 \%$ \\
\hline VA & 104,667 & 1,056 & $31.6 \%$ & 3,342 & 77 & 3,265 & 1,025 & $31.4 \%$ \\
\hline VT & 14,064 & 996 & $38.0 \%$ & 2,621 & 25 & 2,596 & 973 & $37.5 \%$ \\
\hline WA & 96,664 & 1,055 & $38.5 \%$ & 2,740 & 144 & 2,596 & 1,019 & $39.3 \%$ \\
\hline $\mathrm{WI}$ & 102,908 & 1,055 & $46.8 \%$ & 2,254 & 32 & 2,222 & 1,022 & $46.0 \%$ \\
\hline WV & 32,669 & 1,033 & $30.5 \%$ & 3,387 & 56 & 3,331 & 888 & $26.7 \%$ \\
\hline WY & 15,579 & 990 & $40.8 \%$ & 2,426 & 108 & 2,318 & 980 & $42.3 \%$ \\
\hline Virgin Islands & 1,599 & 621 & $25.7 \%$ & 1,026 & 135 & 891 & 157 & $17.6 \%$ \\
\hline Guam & 1,200 & 512 & $23.9 \%$ & 745 & 126 & 619 & 143 & $23.1 \%$ \\
\hline $\begin{array}{l}\text { Northern } \\
\text { Mariana Islands }\end{array}$ & 2,297 & 640 & $20.6 \%$ & 465 & 97 & 368 & 70 & $19.0 \%$ \\
\hline TOTAL & $4,639,548$ & 53,786 & $34.3 \%$ & 153,920 & 5,236 & 148,684 & 48,704 & $32.8 \%$ \\
\hline
\end{tabular}




\section{TABLE 2}

\section{Number of Active LPN/LVN Licenses}

\begin{tabular}{|c|c|c|c|c|c|c|c|c|}
\hline Jurisdiction & $\begin{array}{l}\text { Number of } \\
\text { Active } \\
\text { LPN/LVN } \\
\text { Licenses }\end{array}$ & $\begin{array}{c}\text { Estimated } \\
\text { Sample: } 95 \% \\
\text { Confidence, } \\
\text { 3\% Error } \\
\end{array}$ & $\begin{array}{c}\text { Estimated } \\
\text { Total } \\
\text { Response } \\
\text { Rate } \\
\end{array}$ & $\begin{array}{l}\text { Number of } \\
\text { Surveys } \\
\text { Mailed }\end{array}$ & Undeliverable & $\begin{array}{l}\text { Number } \\
\text { Received }\end{array}$ & $\begin{array}{l}\text { Number of } \\
\text { Responses }\end{array}$ & $\begin{array}{c}\text { Total } \\
\text { Response } \\
\text { Rate }\end{array}$ \\
\hline AK & 865 & 446 & $30.7 \%$ & 636 & 39 & 597 & 148 & $24.8 \%$ \\
\hline $\mathrm{AL}$ & 18,486 & 1,005 & $28.6 \%$ & 3,514 & 125 & 3,389 & 926 & $27.3 \%$ \\
\hline AR & 15,287 & 998 & $28.0 \%$ & 3,564 & 98 & 3,466 & 875 & $25.2 \%$ \\
\hline AZ & 10,362 & 968 & $24.2 \%$ & 4,000 & 403 & 3,597 & 954 & $26.5 \%$ \\
\hline CA & 100,129 & 1,056 & $22.5 \%$ & 4,693 & 106 & 4,587 & 1,079 & $23.5 \%$ \\
\hline $\mathrm{CO}$ & 8,712 & 940 & $25.4 \%$ & 3,701 & 390 & 3,311 & 1,018 & $30.7 \%$ \\
\hline CT & 12,956 & 987 & $23.0 \%$ & 4,291 & 256 & 4,035 & 870 & $21.6 \%$ \\
\hline DC & 2,205 & 761 & $22.8 \%$ & 1,827 & 33 & 1,794 & 357 & $19.9 \%$ \\
\hline $\mathrm{DE}$ & 3,050 & 774 & $27.6 \%$ & 2,693 & 145 & 2,548 & 594 & $23.3 \%$ \\
\hline $\mathrm{FL}$ & 64,237 & 1,051 & $23.9 \%$ & 4,397 & 208 & 4,189 & 834 & $19.9 \%$ \\
\hline GA & 30,813 & 1,035 & $24.1 \%$ & 4,295 & 105 & 4,190 & 947 & $22.6 \%$ \\
\hline $\mathrm{HI}$ & 3,004 & 978 & $30.7 \%$ & 2,569 & 189 & 2,380 & 713 & $30.0 \%$ \\
\hline IA & 10,517 & 972 & $32.0 \%$ & 3,038 & 98 & 2,940 & 931 & $31.7 \%$ \\
\hline ID & 3,747 & 818 & $32.7 \%$ & 2,502 & 86 & 2,416 & 759 & $31.4 \%$ \\
\hline $\mathrm{IL}$ & 26,820 & 1,030 & $25.0 \%$ & 4,120 & 79 & 4,041 & 1,101 & $27.2 \%$ \\
\hline IN & 25,385 & 1,021 & $27.5 \%$ & 3,713 & 97 & 3,616 & 905 & $25.0 \%$ \\
\hline KS & 10,639 & 969 & $32.3 \%$ & 3,000 & 141 & 2,859 & 817 & $28.6 \%$ \\
\hline KY & 13,835 & 992 & $25.4 \%$ & 3,906 & 85 & 3,821 & 931 & $24.4 \%$ \\
\hline LA & 23,160 & 1,020 & $22.7 \%$ & 4,493 & 69 & 4,424 & 858 & $19.4 \%$ \\
\hline MA & 20,488 & 1,018 & $25.9 \%$ & 3,931 & 135 & 3,796 & 896 & $23.6 \%$ \\
\hline MD & 12,297 & 983 & $23.2 \%$ & 4,237 & 211 & 4,026 & 949 & $23.6 \%$ \\
\hline ME & 2,242 & 730 & $33.4 \%$ & 2,090 & 67 & 2,023 & 728 & $36.0 \%$ \\
\hline $\mathrm{MI}$ & 24,237 & 1,024 & $33.9 \%$ & 3,021 & 82 & 2,939 & 904 & $30.8 \%$ \\
\hline $\mathrm{MN}$ & 22,297 & 1,018 & $38.3 \%$ & 2,658 & 62 & 2,596 & 1,043 & $40.2 \%$ \\
\hline MS & 14,222 & 989 & $20.9 \%$ & 4,732 & 258 & 4,474 & 1,041 & $23.3 \%$ \\
\hline MT & 2,663 & 791 & $42.0 \%$ & 1,883 & 114 & 1,769 & 770 & $43.5 \%$ \\
\hline NC & 22,605 & 1,018 & $29.8 \%$ & 3,416 & 158 & 3,258 & 987 & $30.3 \%$ \\
\hline ND & 3,161 & 805 & $39.2 \%$ & 2,054 & 79 & 1,975 & 814 & $41.2 \%$ \\
\hline $\mathrm{NE}$ & 5,842 & 918 & $35.6 \%$ & 2,579 & 109 & 2,470 & 882 & $35.7 \%$ \\
\hline $\mathrm{NH}$ & 3,130 & 796 & $29.8 \%$ & 1,379 & 76 & 1,303 & 347 & $26.6 \%$ \\
\hline $\mathrm{NJ}$ & 23,435 & 1,021 & $25.5 \%$ & 4,004 & 82 & 3,922 & 912 & $23.3 \%$ \\
\hline NM & 2,758 & 768 & $29.0 \%$ & 2,648 & 180 & 2,468 & 727 & $29.5 \%$ \\
\hline NV & 3,905 & 832 & $27.5 \%$ & 3,025 & 252 & 2,773 & 741 & $26.7 \%$ \\
\hline$N Y$ & 76,928 & 1,052 & $23.9 \%$ & 1,903 & 80 & 1,823 & 356 & $19.5 \%$ \\
\hline $\mathrm{OH}$ & 54,720 & 1,046 & $28.8 \%$ & 3,632 & 163 & 3,469 & 1,017 & $29.3 \%$ \\
\hline OK & 16,808 & 1,012 & $26.1 \%$ & 3,877 & 193 & 3,684 & 790 & $21.4 \%$ \\
\hline OR & 5,381 & 884 & $29.0 \%$ & 3,048 & 151 & 2,897 & 860 & $29.7 \%$ \\
\hline PA & 53,989 & 1,045 & $34.9 \%$ & 2,994 & 68 & 2,926 & 894 & $30.6 \%$ \\
\hline $\mathrm{RI}$ & 2,008 & 691 & $27.4 \%$ & 1,873 & 96 & 1,777 & 480 & $27.0 \%$ \\
\hline $\mathrm{SC}$ & 12,682 & 980 & $27.2 \%$ & 3,603 & 155 & 3,448 & 856 & $24.8 \%$ \\
\hline SD & 2,617 & 753 & $35.8 \%$ & 2,103 & 62 & 2,041 & 732 & $35.9 \%$ \\
\hline TN & 30,216 & 1,031 & $25.3 \%$ & 4,075 & 100 & 3,975 & 926 & $23.3 \%$ \\
\hline$T X$ & 105,655 & 1,056 & $23.7 \%$ & 4,456 & 210 & 4,246 & 900 & $21.2 \%$ \\
\hline UT & 2,834 & 769 & $29.3 \%$ & 2,625 & 0 & 2,625 & 553 & $21.1 \%$ \\
\hline VA & 27,745 & 1,028 & $22.6 \%$ & 4,549 & 157 & 4,392 & 1,003 & $22.8 \%$ \\
\hline VT & 2,379 & 699 & $31.2 \%$ & 1,868 & 60 & 1,808 & 471 & $26.1 \%$ \\
\hline WA & 11,513 & 978 & $30.8 \%$ & 3,175 & 269 & 2,906 & 836 & $28.8 \%$ \\
\hline $\mathrm{WI}$ & 13,166 & 999 & $42.7 \%$ & 2,340 & 23 & 2,317 & 921 & $39.7 \%$ \\
\hline
\end{tabular}




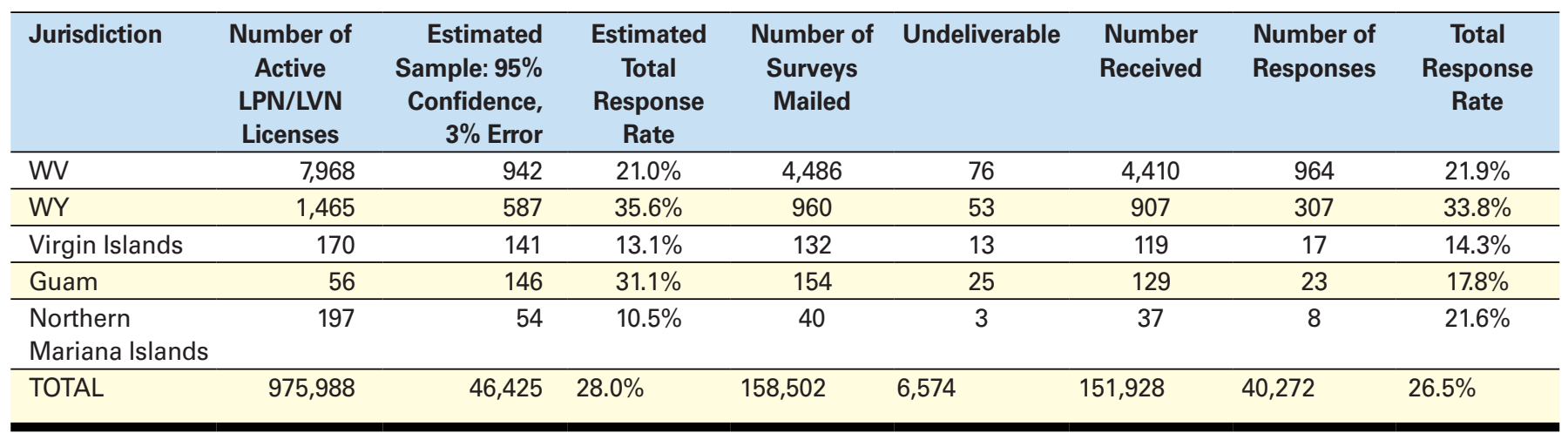

\section{Survey Instrument and Materials}

NCSBN and the Forum currently use the Nurse Supply Minimum Data Set (MDS), which was approved in 2009 after an intensive process of consensus-building and data compilation, to collect data on the nursing workforce at the state level. Because of the evolution and transformation of health care and nursing that have taken place since 2009, in 2015 the Forum made the decision to revisit the Supply MDS. Throughout 2015, the Forum's Research Committee undertook the task of reviewing the Supply MDS to ensure that the elements included were still relevant. This review included an assessment of items that were currently being collected by states and national organizations in their supply data collection efforts. The revised elements of this assessment were subject to review by members of the research committee as well as review and approval of all Forum member executive directors. Additionally, the Forum solicited comment on the proposed changes to the Supply MDS from national organizations and health care workforce researchers. Feedback received was incorporated into the updated version of the Supply MDS, which was ratified by the Forum in 2016.

Data were collected between August 28, 2017, and January 15, 2018. The survey was a four-page Scantron ${ }^{\circledR}$ fillable document with 31 questions. Data elements from the latest revision of the Forum's Supply MDS were incorporated, resulting in the following changes to the survey between the 2015 and 2017 waves of data collection:

- Ethnicity was asked separately from race

- Highest level of nursing education and highest level of non-nursing education were asked separately

- Response options for employment status, employment setting, and employment specialty were added, deleted, or revised.

\section{Procedure}

Western Institutional Review Board granted approval for the current study. A unique identification number was generated and assigned to each sampled participant. The identification was only to be used to record that the survey had been returned. This helped prevent unnecessary and expensive duplicate mailings to those selected to participate in the study. Also, a unique access code identifier was used for the online survey option. Electronic versions of the data were kept on department-secured servers through the end of the project. NCSBN's research staff, three key members of the Forum, and key personnel at Scantron had initial access to the identifiable data. Scantron no longer has access to the identifiable data.

Once materials were developed and the sampling file was complete, surveys were distributed over a 20 -week period that included the following steps:

- Week 1: An announcement letter was sent to all nurses selected to participate (except for those in Utah, whose sampling process was delayed). The letter included a URL and access code to take the survey online.

- Week 2: Nurses with email addresses were sent a link to take the survey online. For nurses without email addresses, a cover letter and paper survey were mailed via first class U.S. mail.

- Week 3: Nurses with email addresses were sent a reminder to take the survey online.

- Week 4: Nurses with email addresses were sent a second reminder to take the survey.

- Week 5: For nurses who had not responded, a cover letter and paper survey were mailed via first class U.S. mail.

- Week 7: Because the sampling process was delayed in Utah, as a supplemental mailing, nurses in this state were mailed a cover letter via first class U.S. mail, which included a URL and access code and a quick response code to take the survey online.

- Week 8: For nurses who still had not responded, an additional cover letter and paper survey were mailed via first class U.S. mail. This mailing included the nurses in Utah.

- Week 13: For nurses in Utah who still had not responded, an additional cover letter and paper survey were mailed via first class U.S. mail.

- Week 20: Deadline for receipt of all surveys.

Once the survey was closed, the final data file was compiled separately for RNs and LPNs/LVNs. 


\section{Analysis}

At the close of the survey, 48,704 of 148,684 successfully delivered RN surveys were returned, for a response rate of 32.8\%; 40,272 of 151,928 successfully delivered LPN/LVN surveys were returned, for a response rate of 26.5\%. A formal nonresponse bias analysis was conducted immediately following the close of the survey. An analysis of basic demographic data (i.e., gender, age, and race/ethnicity) for all RN licensees sampled from the Nursys database was used to compare survey respondents to survey nonrespondents. Results revealed the following groups of nurses may have been slightly overrepresented in both the RN and LPN/LVN samples: White/Caucasian, female, and age 50 or older. Because of missing or incomplete data on race/ethnicity, only gender and age were used to make nonresponse weighting adjustments. Additionally, since sampling was stratified by state, to prevent smaller states from being overrepresented in the overall analysis, a weighting variable was constructed to adjust for differing nursing population sizes across states. A description of this process can be found in Appendices A and B.

To help the readers get an accurate and comprehensive view of the statistics drawn from the sample, the number of actual valid answers to each question is reported for every table. Missing data were not imputed; hence, the presented statistics represent the actual responses from participants who responded to each respective survey item. If a participant did not respond to a certain item, they were not part of the analysis for that item. Additionally, some tables display data for all responding nurse licensees while other tables display data for employed nurses. If a table is specific to nurses employed in nursing, it is explicitly stated. The data for Utah nurses was sampled from a file of nurses living in Utah. This differs from the data collected for the rest of the jurisdictions, which were sampled from files of nurses licensed in those jurisdictions. Many tables include bar graphs to help you easily visualize and comprehend the data presented.

\section{Population Estimate}

For each question on the survey, frequencies are shown that reflect the nonresponse weighting adjustments. For some of the questions, an additional set of frequencies are shown that display the data scaled up to reflect estimates of the nursing population in the subgroup identified. For example, the 354,449 males reported in the 2017 column of Table 3 reflect the number of male respondents adjusted to the population level of number of RNs after the nonresponse weighting adjustments were made to the survey results and after adjusting the population number to account for nurses having licenses in multiple states.

The adjustments for multiple licenses were derived from data in the Nursys database in February 2018. At that time, the database contained 4,726,967 RN licenses, which corresponded to 4,025,446 unique RNs. This produced an RN adjustment factor of $4,025,446 / 4,726,967=0.851592$, which when applied to the 4,639,548 licenses in the RN sample frame (see Table 1) produced an estimate of 3,951,001 unique RNs in 2017.

For LPNs/LVNs, the February 2018 database contained 971,386 licenses, which corresponded to 920,013 unique LPNs/LVNs. This produced an LPN/LVN adjustment factor of 920,013/971,386 = 0.947114, which when applied to the 975,988 licenses in the LPN/LVN sample frame (see Table 2) produced an estimate of 920,743 unique LPNs/LVNs in 2017. 


\section{Registered Nurse Results}

\section{Gender}

While the nursing workforce continues to be predominately female (90.9\%), the proportion of respondents who were male increased 2.5 percentage points from 2013 to 2017. See Table 3.

\section{TABLE 3}

\section{Gender Distribution of Nurses-RN}

\begin{tabular}{|c|c|c|c|c|c|c|}
\hline \multicolumn{7}{|c|}{ Weighted Sample Values } \\
\hline & \multicolumn{2}{|c|}{$2013(n=40,364.5)$} & \multicolumn{2}{|c|}{$2015(n=43,330.9)$} & \multicolumn{2}{|c|}{$2017(n=48,084.9)$} \\
\hline & $n$ & Percent & $n$ & Percent & $n$ & Percent \\
\hline Female & $37,685.6$ & $93.4 \%$ & $39,871.4$ & $92.0 \%$ & $43,715.5$ & $90.9 \%$ \\
\hline
\end{tabular}

\begin{tabular}{|c|c|c|c|c|c|c|}
\hline \multicolumn{7}{|c|}{ Estimated Population Values } \\
\hline & Freq & Percent & Freq & Percent & Freq & Percent \\
\hline Female & $3,114,767$ & $93.4 \%$ & $3,198,650$ & $92.0 \%$ & $3,546,320$ & $90.9 \%$ \\
\hline
\end{tabular}

\section{Race/Ethnicity}

Nearly $81 \%$ of responding RNs were White/Caucasian. An additional 7.5\% identified as Asian, 6.2\% identified as Black/African American, $2.9 \%$ identified as other, and $1.7 \%$ identified as two or more races. See Table 4.

One of the changes to the 2017 survey included the modification of the race and ethnicity items. Previously, race and ethnicity categories were listed in one question. The question was modified, so that ethnicity and race are now two separate questions. Respondents were first asked whether they were of Hispanic or Latino origin and then asked to identify their race. Anyone who selected more than one race category was recoded into the two or more races category. RNs indicating that they were of Hispanic or Latino origin increased by 1.9 percentage points between 2013 and 2017. See Table 5.

\section{TABLE 4}

\section{Race/Ethnicity-RN}

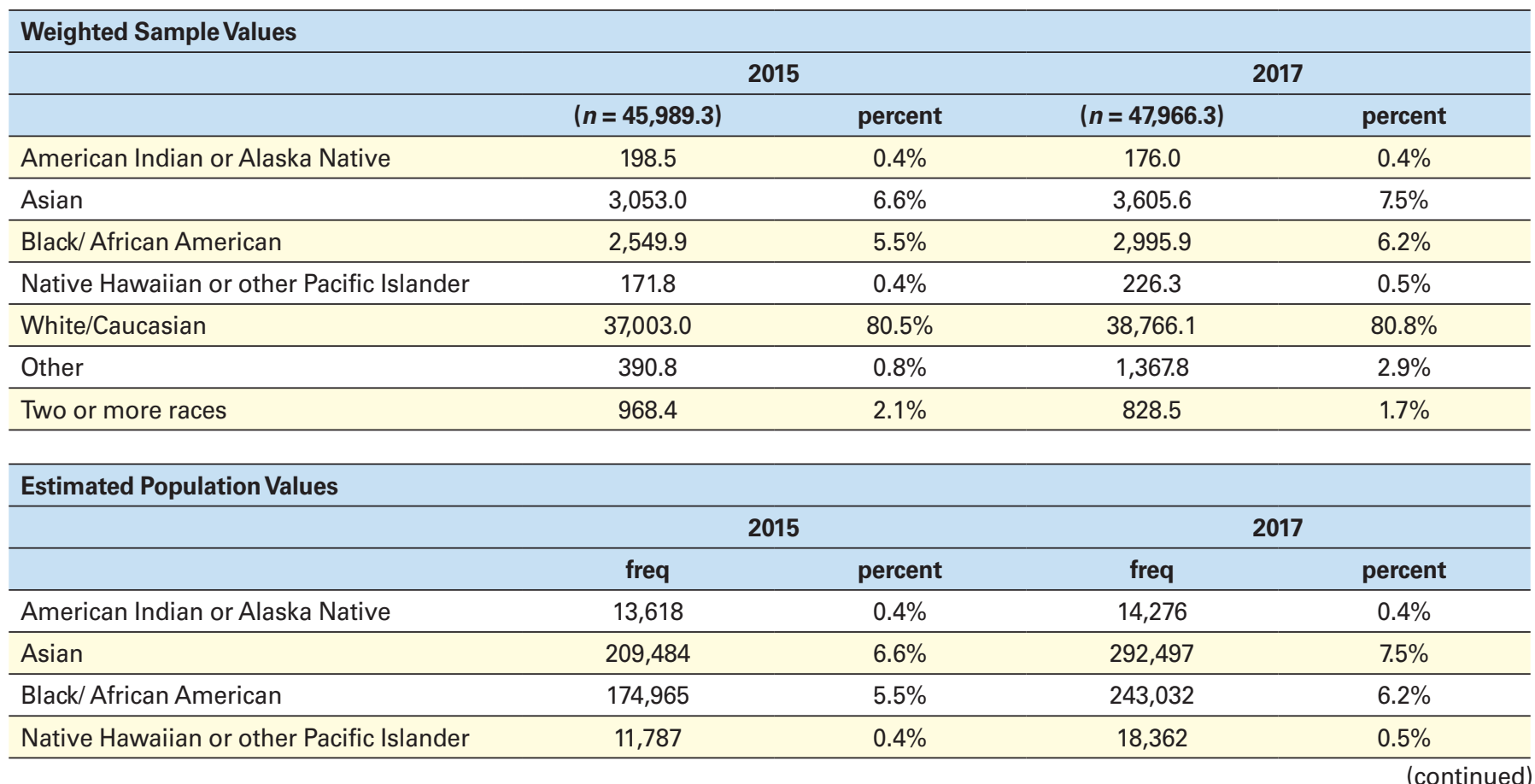




\section{Race/Ethnicity-RN (continued)}

\section{Estimated Population Values}

\begin{tabular}{|c|c|c|c|c|}
\hline & \multicolumn{2}{|c|}{2015} & \multicolumn{2}{|c|}{2017} \\
\hline & freq & percent & freq & percent \\
\hline White/Caucasian & $2,539,013$ & $80.5 \%$ & $3,144,812$ & $80.8 \%$ \\
\hline Two or more races & 77,687 & $<0.1 \%$ & 67,214 & $1.7 \%$ \\
\hline
\end{tabular}

Note. In the 2013 and 2015 surveys, the Hispanic/Latino origin and race categories were combined into one question. For the race question, respondents were asked to select all that apply. The responses were subsequently recoded to ensure that the race categories were mutually exclusive. Respondents selecting multiple race categories were reclassified into the 'Two or more races' category.

\section{TABLE 5}

\section{Ethnicity of Hispanic or Latino Origin-RN}

\begin{tabular}{|c|c|c|c|c|c|c|}
\hline \multicolumn{7}{|l|}{ Weighted Sample Values } \\
\hline & \multicolumn{2}{|c|}{$2013(n=41,876.0)$} & \multicolumn{2}{|c|}{$2015(n=45,989.3)$} & \multicolumn{2}{|c|}{$2017(n=47,965.6)$} \\
\hline & $n$ & Percent & $n$ & Percent & $n$ & Percent \\
\hline Not of Hispanic or Latino origin & $40,469.5$ & $96.6 \%$ & $44,335.3$ & $96.4 \%$ & $45,324.5$ & $94.7 \%$ \\
\hline Hispanic or Latino origin & 116,257 & $3.4 \%$ & 132,692 & $3.6 \%$ & 205,086 & $5.3 \%$ \\
\hline Not of Hispanic or Latino origin & $3,344,857$ & $96.6 \%$ & $3,556,764$ & $96.4 \%$ & $3,676,844$ & $94.7 \%$ \\
\hline $\begin{array}{l}\text { Note. In the } 2013 \text { and } 2015 \text { surveys, } \\
\text { dents were asked to select all that a } \\
\text { spondents selecting multiple race ce }\end{array}$ & $\begin{array}{l}\text { ino origin anc } \\
\text { ses were subs } \\
\text { eclassified int }\end{array}$ & $\begin{array}{l}\text { categories } \\
\text { ently recod } \\
\text { 'Two or } \mathrm{m}\end{array}$ & $\begin{array}{l}\text { combined ir } \\
\text { ensure that } t \\
\text { ces' category }\end{array}$ & $\begin{array}{l}\text { e questior } \\
\text { categori }\end{array}$ & $\begin{array}{l}\text { the race que } \\
\text { re mutually }\end{array}$ & $\begin{array}{l}\text { respon- } \\
\text { ive. Re- }\end{array}$ \\
\hline
\end{tabular}

\section{Race/ethnicity by gender}

Race/ethnicity groups with the highest percent of males are Hispanic/Latino (15.9\%), Asian (15.7\%), and Native Hawaiian or other Pacific Islander groups (15.2\%). These groups had a substantially higher percent of males than the overall average (9.1\%). See Table 6.

TABLE 6

\section{Race/Ethnicity by Gender-RN}

\begin{tabular}{|c|c|c|c|}
\hline \multicolumn{4}{|l|}{ Weighted Sample Values } \\
\hline & Male & Female & $n$ \\
\hline American Indian or Alaska Native & 22.6 & 136.5 & 159.1 \\
\hline \multirow[b]{2}{*}{ Asian } & 560.4 & $3,001.2$ & $3,561.6$ \\
\hline & $15.7 \%$ & $84.3 \%$ & \\
\hline \multirow{2}{*}{ Black/African American } & 241.3 & $2,683.7$ & $2,925.0$ \\
\hline & 30.0 & 167.0 & 197.0 \\
\hline \multirow[t]{2}{*}{ Native Hawaiian or other Pacific Islander } & $15.2 \%$ & $84.8 \%$ & \\
\hline & $2,915.3$ & $34,219.6$ & $37,134.9$ \\
\hline \multirow[t]{2}{*}{ White/Caucasian } & $7.9 \%$ & $92.1 \%$ & \\
\hline & 400.3 & $2,120.1$ & $2,520.4$ \\
\hline
\end{tabular}




\begin{tabular}{|c|c|c|c|}
\hline Weighted Sample Values & & & \\
\hline & Male & Female & $n$ \\
\hline & 85.6 & 563.9 & 649.6 \\
\hline \multirow[t]{2}{*}{ Other } & $13.2 \%$ & $86.8 \%$ & \\
\hline & 91.3 & 618.1 & 709.4 \\
\hline \multirow[t]{2}{*}{ Two or more races } & $12.9 \%$ & $87.1 \%$ & \\
\hline & $4,346.8$ & $43,510.2$ & $47,857.0$ \\
\hline Total & $9.1 \%$ & $90.9 \%$ & \\
\hline
\end{tabular}

Note. In the 2013 and 2015 surveys, the Hispanic/Latino origin and race categories were combined into one question. For the race question, respondents were asked to select all that apply. The responses were subsequently recoded to ensure that the race categories were mutually exclusive. Respondents selecting multiple race categories were reclassified into the 'Two or more races' category.

\section{Age}

In 2017, the median age of RN respondents was 53 years. This is unchanged from the median age of RN respondents in 2015 , which was also 53 years, and only a year older than the median age of 52 from 2013.

As shown in Table 7, $50.9 \%$ of respondents were age 50 or older, which is consistent with the results from 2015 when $50 \%$ of respondents were age 50 or older. There was a 2.2 percentage point increase between 2015 and 2017 for respondents reporting that they were age 65 or older.

\section{TABLE 7}

\section{Age Distribution-RN}

\begin{tabular}{|c|c|c|c|c|c|c|}
\hline \multicolumn{7}{|c|}{ Weighted Sample Values } \\
\hline \multirow[b]{2}{*}{ Age } & \multicolumn{2}{|c|}{$2013(n=34,793.9)$} & \multicolumn{2}{|c|}{$2015(n=41,258.6)$} & \multicolumn{2}{|c|}{$2017(n=47,527.3)$} \\
\hline & $n$ & Percent & $n$ & Percent & $n$ & Percent \\
\hline $30-34$ & $2,615.4$ & $7.5 \%$ & $4,098.0$ & $9.9 \%$ & $4,762.8$ & $10.0 \%$ \\
\hline $35-39$ & $2,784.0$ & $8.0 \%$ & $3,928.1$ & $9.5 \%$ & $4,390.6$ & $9.2 \%$ \\
\hline $45-49$ & $3,379.3$ & $9.7 \%$ & $4,398.2$ & $10.7 \%$ & $5,250.7$ & $11.1 \%$ \\
\hline $50-54$ & $4,652.6$ & $13.4 \%$ & $4,724.8$ & $11.5 \%$ & $4,914.9$ & $10.3 \%$ \\
\hline $55-59$ & $5,887.7$ & $16.9 \%$ & $5,622.4$ & $13.6 \%$ & $5,834.4$ & $12.3 \%$ \\
\hline $60-64$ & $4,570.1$ & $13.1 \%$ & $5,254.9$ & $12.7 \%$ & $6,489.8$ & $13.7 \%$ \\
\hline$\geq 65$ & $4,818.4$ & $13.9 \%$ & $5,126.3$ & $12.4 \%$ & $6,932.9$ & $14.6 \%$ \\
\hline
\end{tabular}

\section{Estimated Population Values}

\begin{tabular}{|c|c|c|c|c|c|c|}
\hline Age & \multicolumn{2}{|c|}{2013} & \multicolumn{2}{|c|}{2015} & \multicolumn{2}{|c|}{2017} \\
\hline$<30$ & 247,778 & $8.6 \%$ & 313,291 & $9.5 \%$ & 372,716 & $9.7 \%$ \\
\hline $30-34$ & 216,164 & $7.5 \%$ & 328,759 & $9.9 \%$ & 386,374 & $10.0 \%$ \\
\hline $35-39$ & 230,098 & $8.0 \%$ & 315,127 & $9.5 \%$ & 356,175 & $9.2 \%$ \\
\hline $40-44$ & 255,281 & $8.9 \%$ & 337,000 & $10.2 \%$ & 353,430 & $9.2 \%$ \\
\hline $50-54$ & 384,543 & $13.4 \%$ & 379,041 & $11.5 \%$ & 398,712 & $10.3 \%$ \\
\hline $55-59$ & 486,625 & $16.9 \%$ & 451,051 & $13.6 \%$ & 473,303 & $12.3 \%$ \\
\hline $60-64$ & 377,724 & $13.1 \%$ & 421,574 & $12.7 \%$ & 526,468 & $13.7 \%$ \\
\hline$\geq 65$ & 398,247 & $13.9 \%$ & 411,256 & $12.4 \%$ & 562,414 & $14.6 \%$ \\
\hline
\end{tabular}

(continued) 


\section{Age Distribution - RN (continued)}

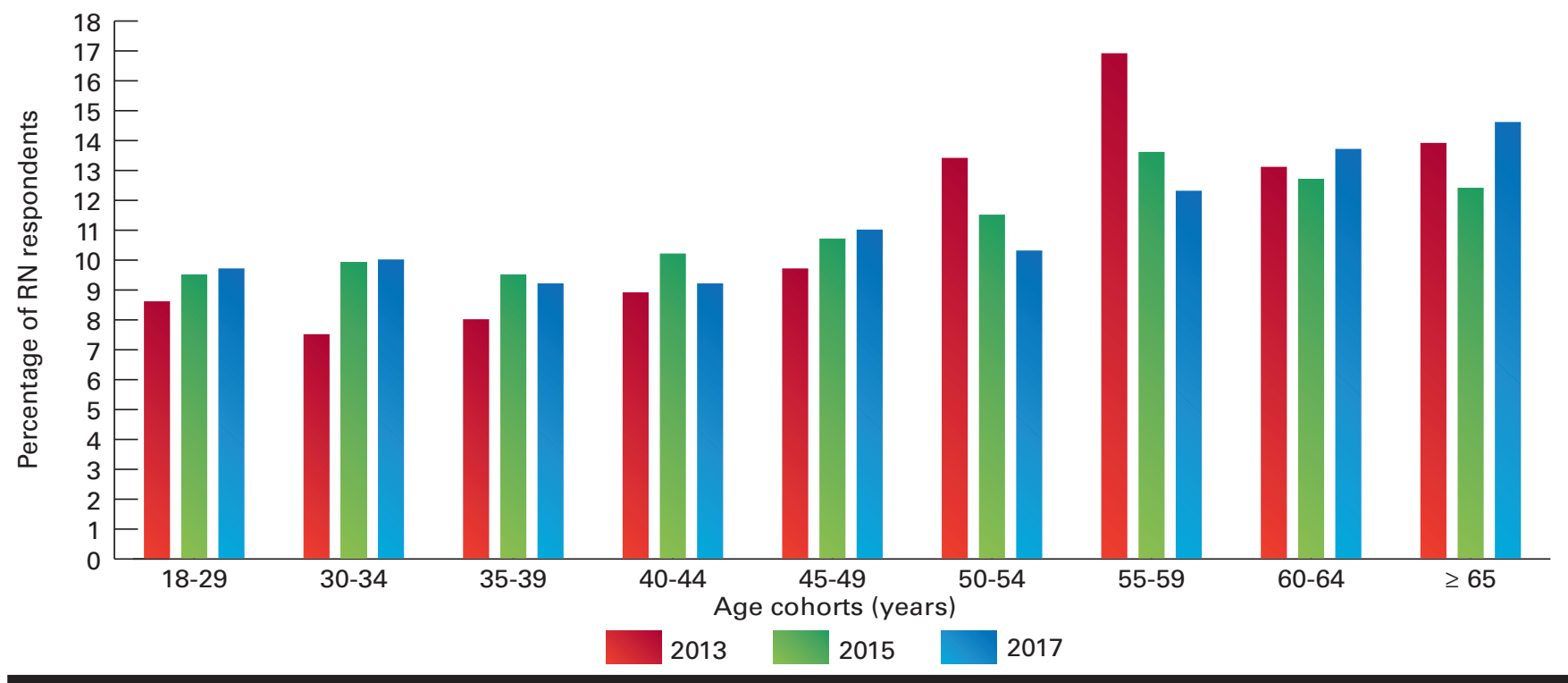

Age by gender

Among older age-groups (ages 50+), women comprise a much higher proportion of the population than younger age-groups (ages 30 49). See Table 8.

\section{TABLE 8}

\section{Age Distribution by Gender-RN}

\begin{tabular}{|c|c|c|c|c|c|c|c|c|c|c|}
\hline \multicolumn{11}{|c|}{ Weighted Sample Values } \\
\hline Age cohort & $<30$ & $30-34$ & $35-39$ & $40-44$ & $45-49$ & $50-54$ & $55-59$ & $60-64$ & $\geq 65$ & $n$ \\
\hline Male & 387.4 & 540.9 & 413.4 & 471.7 & 704.7 & 401.6 & 454.0 & 493.1 & 407.0 & $4,273.9$ \\
\hline \multirow[t]{2}{*}{ Female } & $4,165.1$ & $4,198.4$ & $3,956.7$ & $3,857.4$ & $4,504.2$ & $4,444.4$ & $5,325.3$ & $5,922.5$ & $6,433.8$ & $42,807.9$ \\
\hline & $9.7 \%$ & $9.8 \%$ & $9.2 \%$ & $9.0 \%$ & $10.5 \%$ & $10.4 \%$ & $12.4 \%$ & $13.8 \%$ & $15.0 \%$ & \\
\hline Total & $9.7 \%$ & $10.1 \%$ & $9.3 \%$ & $9.2 \%$ & $11.1 \%$ & $10.3 \%$ & $12.3 \%$ & $13.6 \%$ & $14.5 \%$ & \\
\hline
\end{tabular}

Age by race/ethnicity

The proportion of Hispanic/Latinos in the $\mathrm{RN}$ workforce has become progressively larger as new nurses have entered the workforce. Among those RNs 65 and older, only $7.3 \%$ are Hispanic/Latino compared to $13.9 \%$ of those under 30 . This indicates an increase in the overall diversity of the nursing population. See Table 9.

\section{TABLE 9}

\section{Age Distribution by Race/Ethnicity-RN}

\begin{tabular}{|c|c|c|c|c|c|c|c|c|c|c|}
\hline \multicolumn{11}{|l|}{ Weighted Sample Values } \\
\hline Age cohort & $<30$ & $30-34$ & $35-39$ & $40-44$ & $45-49$ & $50-54$ & $55-59$ & $60-64$ & $\geq 65$ & $n$ \\
\hline $\begin{array}{l}\text { American Indian or Alaska } \\
\text { Native }\end{array}$ & 12.1 & 8.8 & 15.1 & 16.2 & 20.2 & 15.6 & 17.7 & 24.0 & 25.9 & 155.6 \\
\hline \multirow[t]{2}{*}{ Asian } & 385.5 & 443.3 & 339.2 & 442.2 & 626.9 & 274.6 & 308.7 & 410.0 & 292.5 & $3,522.9$ \\
\hline & $10.9 \%$ & $12.6 \%$ & $9.6 \%$ & $12.6 \%$ & $17.8 \%$ & $7.8 \%$ & $8.8 \%$ & $11.6 \%$ & $8.3 \%$ & \\
\hline
\end{tabular}




\begin{tabular}{|c|c|c|c|c|c|c|c|c|c|c|}
\hline \multicolumn{11}{|l|}{ Weighted Sample Values } \\
\hline Age cohort & $<30$ & $30-34$ & $35-39$ & $40-44$ & $45-49$ & $50-54$ & $55-59$ & $60-64$ & $\geq 65$ & $n$ \\
\hline Black/African American & 184.1 & 256.7 & 373.2 & 361.6 & 418.8 & 348.8 & 290.4 & 252.7 & 384.5 & $2,870.9$ \\
\hline \multirow{2}{*}{$\begin{array}{l}\text { Native Hawaiian or other } \\
\text { Pacific Islander }\end{array}$} & 13.6 & 46.4 & 12.6 & 20.8 & 19.0 & 22.3 & 18.4 & 23.0 & 18.4 & 194.5 \\
\hline & $7.0 \%$ & $23.9 \%$ & $6.5 \%$ & $10.7 \%$ & $9.8 \%$ & $11.5 \%$ & $9.5 \%$ & $11.8 \%$ & $9.5 \%$ & \\
\hline White/Caucasian & $9.5 \%$ & $9.5 \%$ & $8.6 \%$ & $8.4 \%$ & $10.0 \%$ & $10.4 \%$ & $13.1 \%$ & $14.7 \%$ & $15.7 \%$ & \\
\hline \multirow[t]{2}{*}{ Hispanic/Latino of any race } & 343.0 & 345.9 & 322.1 & 301.4 & 289.8 & 243.6 & 237.3 & 200.1 & 179.4 & $2,462.6$ \\
\hline & $13.9 \%$ & $14.0 \%$ & $13.1 \%$ & $12.2 \%$ & $11.8 \%$ & $9.9 \%$ & $9.6 \%$ & $8.1 \%$ & $7.3 \%$ & \\
\hline \multirow[t]{2}{*}{ Other } & 34.3 & 37.8 & 77.4 & 51.4 & 68.1 & 63.1 & 60.9 & 107.7 & 128.7 & 629.5 \\
\hline & $5.4 \%$ & $6.0 \%$ & $12.3 \%$ & $8.2 \%$ & $10.8 \%$ & $10.0 \%$ & $9.7 \%$ & $17.1 \%$ & $20.4 \%$ & \\
\hline Total & $9.7 \%$ & $10.1 \%$ & $9.3 \%$ & $9.2 \%$ & $11.0 \%$ & $10.3 \%$ & $12.3 \%$ & $13.7 \%$ & $14.5 \%$ & \\
\hline
\end{tabular}

Note. In the 2013 and 2015 surveys, the Hispanic/Latino origin and race categories were combined into one question. For the race question, respondents were asked to select all that apply. The responses were subsequently recoded to ensure that the race categories were mutually exclusive. Respondents selecting multiple race categories were reclassified into the 'Two or more races' category.

\section{Education}

Type of nursing degree or credential for first U.S. nursing license

The percentage of nurses reporting the baccalaureate as the nursing degree that qualified them for their first U.S. nursing license increased by 6.3 percentage points between 2013 and 2017. The percent of respondents who initially received a diploma or associate degree decreased by 7.2 percentage points. See Table 10 .

\section{TABLE 10}

\section{Type of Nursing Degree or Credential for First U.S. Nursing License-RN}

\begin{tabular}{|c|c|c|c|c|c|c|}
\hline \multicolumn{7}{|l|}{ Weighted Sample Values } \\
\hline & \multicolumn{2}{|c|}{$2013(n=41,792.7)$} & \multicolumn{2}{|c|}{$2015(n=45,818.9)$} & \multicolumn{2}{|c|}{$2017(n=47,650.0)$} \\
\hline & $n$ & Percent & $n$ & Percent & $n$ & Percent \\
\hline Diploma & $7,364.6$ & $17.4 \%$ & $6,539.3$ & $14.3 \%$ & $5,708.1$ & $12.0 \%$ \\
\hline Associate degree & $16,152.0$ & $38.2 \%$ & $17,625.9$ & $38.5 \%$ & $17,332.5$ & $36.4 \%$ \\
\hline Master's degree & $1,218.0$ & $2.9 \%$ & $1,297.9$ & $2.8 \%$ & $1,836.0$ & $3.9 \%$ \\
\hline
\end{tabular}

\begin{tabular}{|c|c|c|c|c|c|c|}
\hline \multicolumn{7}{|l|}{ Estimated Population Values } \\
\hline & \multicolumn{2}{|c|}{2013} & \multicolumn{2}{|c|}{2015} & \multicolumn{2}{|c|}{2017} \\
\hline & Freq & Percent & Freq & Percent & Freq & Percent \\
\hline Diploma & 608,692 & $17.4 \%$ & 524,607 & $14.3 \%$ & 463,060 & $12.0 \%$ \\
\hline Associate degree & $1,334,987$ & $38.2 \%$ & $1,414,020$ & $38.5 \%$ & $1,406,062$ & $36.4 \%$ \\
\hline Master's degree & 100,673 & $2.9 \%$ & 104,121 & $2.8 \%$ & 148,942 & $3.9 \%$ \\
\hline
\end{tabular}




\section{Type of Nursing Degree or Credential for First U.S. Nursing License-RN (continued)}

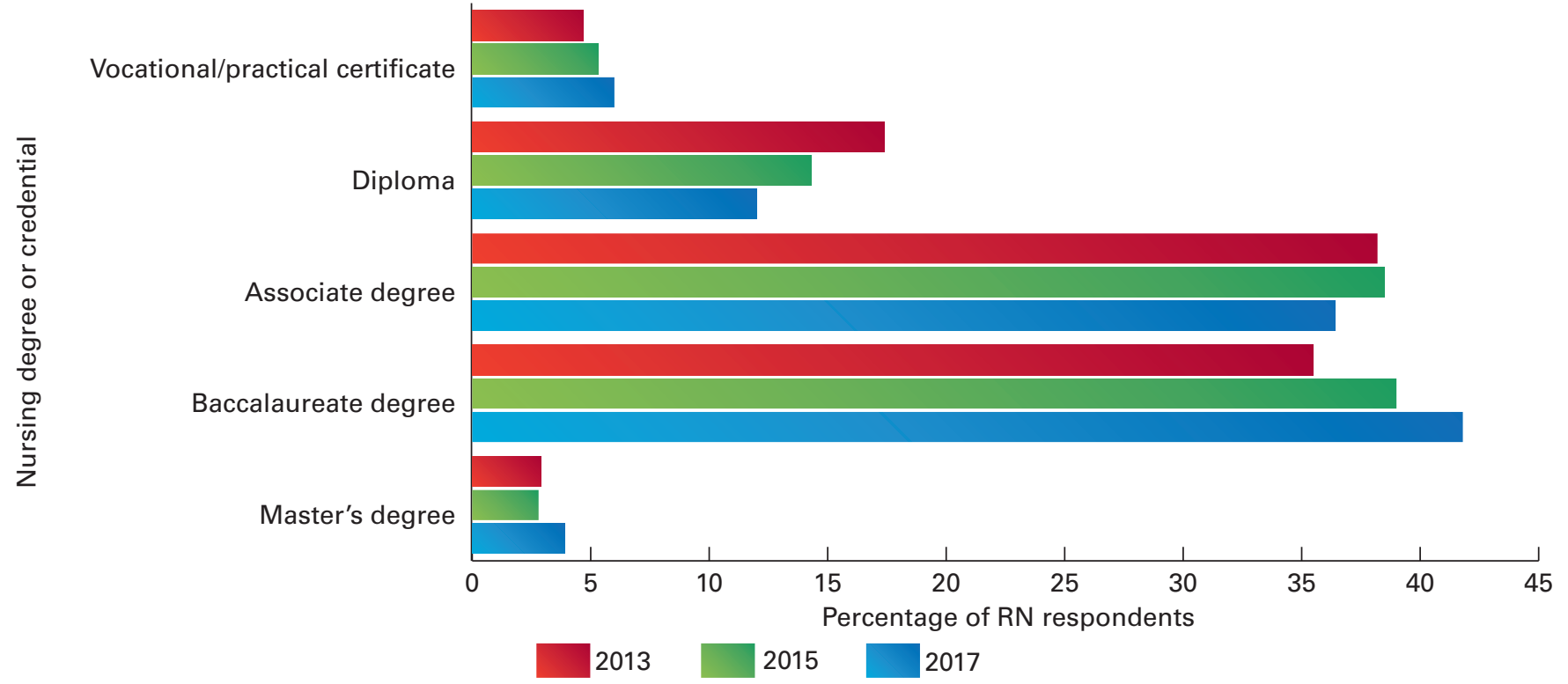

Type of nursing degree or credential for first U.S. nursing license by age. Two degrees are more associated with older RNs: diploma (almost $40 \%$ age 65 and over) and associate degree (12.3\% age 65 and over). Increasingly, a baccalaureate is more common in younger age-groups as the educational qualifier for the nurse's initial licensure $(15.6 \%$ age $<30)$. This indicates that the workforce is becoming increasingly educated. See Table 11.

\section{TABLE 11}

Type of Nursing Degree or Credential for First U.S. Nursing License by Age-RN

\begin{tabular}{|c|c|c|c|c|c|c|c|c|c|c|}
\hline \multicolumn{11}{|c|}{ Weighted Sample Values } \\
\hline Age cohort & $<30$ & $30-34$ & $35-39$ & $40-44$ & $45-49$ & $50-54$ & $55-59$ & $60-64$ & $\geq 65$ & $n$ \\
\hline $\begin{array}{l}\text { Vocational/practical } \\
\text { certificate }\end{array}$ & 90.6 & 226.7 & 245.2 & 244.7 & 327.9 & 309.7 & 420.0 & 437.2 & 510.2 & $2,812.4$ \\
\hline \multirow[t]{2}{*}{ Diploma } & 79.9 & 113.0 & 194.4 & 176.0 & 316.0 & 520.7 & 745.4 & $1,200.3$ & $2,201.7$ & $5,547.5$ \\
\hline & $1.4 \%$ & $2.0 \%$ & $3.5 \%$ & $3.2 \%$ & $5.7 \%$ & $9.4 \%$ & $13.4 \%$ & $21.6 \%$ & $39.7 \%$ & \\
\hline Associate degree & $7.4 \%$ & $9.1 \%$ & $9.9 \%$ & $10.4 \%$ & $12.4 \%$ & $11.3 \%$ & $13.1 \%$ & $14.1 \%$ & $12.3 \%$ & \\
\hline \multirow[t]{2}{*}{ Baccalaureate degree } & $3,040.1$ & $2,602.6$ & $2,010.9$ & $1,898.6$ & $2,187.4$ & $1,871.2$ & $2,110.4$ & $2,089.6$ & $1,732.9$ & $19,543.8$ \\
\hline & $15.6 \%$ & $13.3 \%$ & $10.3 \%$ & $9.7 \%$ & $11.2 \%$ & $9.6 \%$ & $10.8 \%$ & $10.7 \%$ & $8.9 \%$ & \\
\hline \multirow[t]{2}{*}{ Master's degree } & 80.8 & 221.5 & 191.3 & 198.3 & 218.8 & 214.0 & 208.5 & 248.9 & 194.0 & $1,776.1$ \\
\hline & $4.5 \%$ & $12.5 \%$ & $10.8 \%$ & $11.2 \%$ & $12.3 \%$ & $12.0 \%$ & $11.7 \%$ & $14.0 \%$ & $10.9 \%$ & \\
\hline
\end{tabular}

\section{Highest level of nursing education}

Previous iterations of this survey asked for the highest level of education with both nursing and non-nursing degrees combined into one question. The 2017 survey asked two separate questions: highest level of nursing education and highest level of non-nursing education. Due to this change, direct comparisons to 2013 and 2015 are not available for non-nursing education. In terms of highest level of nursing education, $64.1 \%$ of respondents indicated they had a baccalaureate or higher degree in nursing.

By comparison, the Center to Champion Nursing in America's Campaign for Action Dashboard reported that in 2016, 54\% of nurses had a baccalaureate or higher degree in nursing (Campaign Progress, 1027). 
A mere $1.1 \%$ of responding RNs reported a Doctorate in Nursing Practice (DNP) as their highest level of nursing education. While still a small percentage, this is double the percent of nurses who reported the degree type in 2015 and double the number of nurses with a PhD. See Table 12.

\section{TABLE 12}

\section{Highest Level of Nursing Education-RN}

\begin{tabular}{|c|c|c|c|c|c|c|}
\hline \multicolumn{7}{|l|}{ Weighted Sample Values } \\
\hline & \multicolumn{2}{|c|}{$2013(n=35,016.5)$} & \multicolumn{2}{|c|}{$2015(n=38,625.9)$} & \multicolumn{2}{|c|}{$2017(n=48,140.7)$} \\
\hline & $n$ & Percent & $n$ & Percent & $n$ & Percent \\
\hline Associate degree & $11,331.6$ & $32.3 \%$ & $11,608.8$ & $30.0 \%$ & $13,729.1$ & $28.5 \%$ \\
\hline Baccalaureate degree & $14,097.0$ & $40.2 \%$ & $16,762.5$ & $43.4 \%$ & $21,744.1$ & $45.2 \%$ \\
\hline Doctoral degree-PhD & 217.0 & $0.6 \%$ & 340.2 & $0.9 \%$ & 284.1 & $0.6 \%$ \\
\hline Doctoral degree-DNP & 142.5 & $0.4 \%$ & 239.1 & $0.6 \%$ & 551.2 & $1.1 \%$ \\
\hline Doctoral degree-nursing other & 62.9 & $0.2 \%$ & 39.0 & $0.1 \%$ & 46.1 & $0.1 \%$ \\
\hline
\end{tabular}

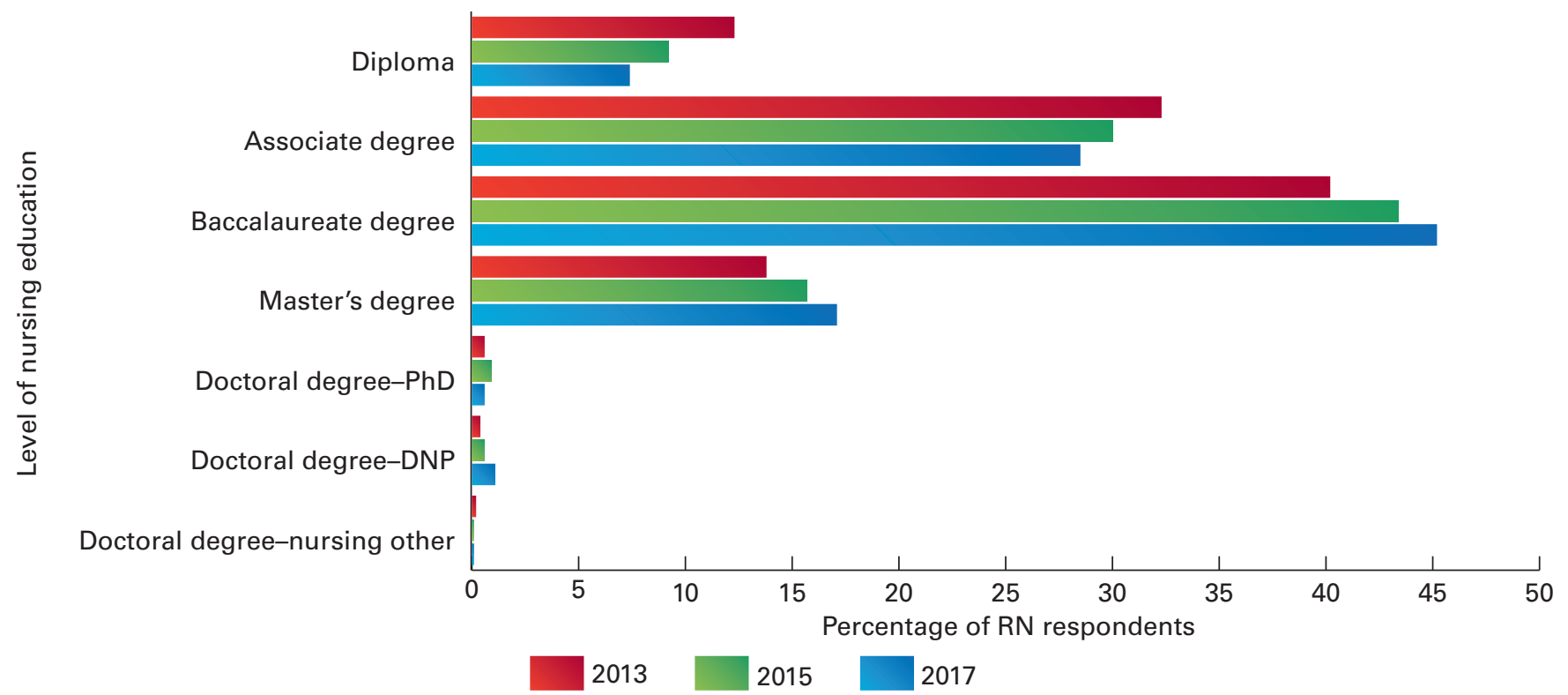

Note. In the 2013 and 2015 surveys, a single question "What is your highest level of education?" was asked, with the set of possible responses including both nursing and non-nursing degrees. In 2017, two separate questions were asked, "Highest level of nursing education" and "Highest level of nonnursing education".

Highest level of nursing education by gender. Of those with a DNP, $14.0 \%$ are men. Conversely, the vast majority of those with a diploma as their highest level of education are women (96.1\%). See Table 13.

\section{TABLE 13}

\section{Highest Level of Nursing Education by Gender-RN}

\begin{tabular}{lccc}
\hline Weighted Sample Values & & & \\
\hline & Male & Female & n \\
\hline Diploma & 137.9 & $3,395.3$ & $3,533.3$ \\
\cline { 2 - 4 } & $3.9 \%$ & $96.1 \%$ & $12,228.6$ \\
\cline { 2 - 4 } & $1,407.0$ & $89.7 \%$ \\
\hline
\end{tabular}

(continued) 


\begin{tabular}{|c|c|c|c|}
\hline \multicolumn{4}{|c|}{ Highest Level of Nursing Education by Gender-RN (continued) } \\
\hline \multicolumn{4}{|c|}{ Weighted Sample Values } \\
\hline & Male & Female & $n$ \\
\hline \multirow[t]{2}{*}{ Baccalaureate degree } & $1,977.5$ & $19,639.1$ & $21,616.6$ \\
\hline & $9.1 \%$ & $90.9 \%$ & \\
\hline \multirow[t]{2}{*}{ Master's degree } & 725.0 & $7,440.9$ & $8,165.9$ \\
\hline & $8.9 \%$ & $91.1 \%$ & \\
\hline \multirow[t]{2}{*}{ Doctoral degree-PhD } & 17.5 & 261.9 & 279.4 \\
\hline & $6.3 \%$ & $93.7 \%$ & \\
\hline \multirow[t]{2}{*}{ Doctoral degree-DNP } & 76.9 & 472.5 & 549.4 \\
\hline & $14.0 \%$ & $86.0 \%$ & \\
\hline \multirow[t]{2}{*}{ Doctoral degree-nursing other } & 1.9 & 44.2 & 46.0 \\
\hline & $4.1 \%$ & $95.9 \%$ & \\
\hline \multirow[t]{2}{*}{ Total } & $4,347.7$ & $43,488.4$ & $47,836.1$ \\
\hline & $9.1 \%$ & $90.9 \%$ & \\
\hline
\end{tabular}

Note. In the 2013 and 2015 surveys, a single question "What is your highest level of education?" was asked, with the set of possible responses including both nursing and non-nursing degrees. In 2017, two separate questions were asked, "Highest level of nursing education" and "Highest level of nonnursing education".

Highest level of nursing education by race. Across all-groups, the most common highest level of education is a baccalaureate, except for American Indian or Alaska Native (45.5\% associate degree). Asian nurses are much more likely to have a baccalaureate degree (65.6\%) as compared to the overall population (45.2\%). See Table 14.

TABLE 14

Highest Level of Nursing Education by Race-RN

\begin{tabular}{|c|c|c|c|c|c|c|c|c|c|}
\hline \multicolumn{10}{|l|}{ Weighted Sample Values } \\
\hline & $\begin{array}{c}\text { Vocational/ } \\
\text { Practical } \\
\text { Certificate }\end{array}$ & Diploma & $\begin{array}{l}\text { Associate } \\
\text { Degree }\end{array}$ & $\begin{array}{l}\text { Baccalaureate } \\
\text { Degree }\end{array}$ & $\begin{array}{l}\text { Master's } \\
\text { Degree }\end{array}$ & $\begin{array}{c}\text { Doctoral } \\
\text { degree } \\
\text { PhD }\end{array}$ & $\begin{array}{c}\text { Doctoral } \\
\text { degree } \\
\text { DNP }\end{array}$ & $\begin{array}{c}\text { Doctoral } \\
\text { degree } \\
\text { nursing other }\end{array}$ & $n$ \\
\hline $\begin{array}{l}\text { American Indian or Alaska } \\
\text { Native }\end{array}$ & $0.0 \%$ & $3.4 \%$ & $45.5 \%$ & $34.7 \%$ & $15.7 \%$ & $0.4 \%$ & $0.4 \%$ & $0.0 \%$ & \\
\hline Asian & 0.0 & 178.8 & 460.3 & $2,338.9$ & 534.9 & 27.2 & 24.1 & 0.5 & $3,564.6$ \\
\hline Black/African American & $0.0 \%$ & $2.6 \%$ & $27.6 \%$ & $50.0 \%$ & $17.5 \%$ & $0.6 \%$ & $1.7 \%$ & $0.1 \%$ & \\
\hline \multirow{2}{*}{$\begin{array}{l}\text { Native Hawaiian or other } \\
\text { Pacific Islander }\end{array}$} & 0.0 & 9.9 & 38.7 & 113.4 & 39.6 & 0.1 & 0.3 & 0.0 & 201.9 \\
\hline & $0.0 \%$ & $4.9 \%$ & $19.2 \%$ & $56.1 \%$ & $19.6 \%$ & $0.0 \%$ & $0.1 \%$ & $0.0 \%$ & \\
\hline \multirow[t]{2}{*}{ White/Caucasian } & 7.9 & $3,130.8$ & $11,046.6$ & $15,774.0$ & $6,437.6$ & 221.3 & 458.6 & 42.6 & $37,119.3$ \\
\hline & $0.0 \%$ & $8.4 \%$ & $29.8 \%$ & $42.5 \%$ & $17.3 \%$ & $0.6 \%$ & $1.2 \%$ & $0.1 \%$ & \\
\hline Other & $0.0 \%$ & $5.5 \%$ & $27.4 \%$ & $44.7 \%$ & $22.1 \%$ & $0.2 \%$ & $0.2 \%$ & $0.0 \%$ & \\
\hline \multirow[t]{2}{*}{ Two or more races } & 0.0 & 18.4 & 186.1 & 368.6 & 125.5 & 1.4 & 4.8 & 0.0 & 704.9 \\
\hline & $0.0 \%$ & $2.6 \%$ & $26.4 \%$ & $52.3 \%$ & $17.8 \%$ & $0.2 \%$ & $0.7 \%$ & $0.0 \%$ & \\
\hline \multirow[t]{2}{*}{ Total } & 9.9 & $3,534.7$ & $13,633.3$ & $21,598.2$ & $8,183.0$ & 277.5 & 550.6 & 45.4 & $47,832.6$ \\
\hline & $0.0 \%$ & $7.4 \%$ & $28.5 \%$ & $45.2 \%$ & $17.1 \%$ & $0.6 \%$ & $1.2 \%$ & $0.1 \%$ & \\
\hline
\end{tabular}

Note. In the 2013 and 2015 surveys, a single question "What is your highest level of education?" was asked, with the set of possible responses including both nursing and non-nursing degrees. In 2017, two separate questions were asked, "Highest level of nursing education" and "Highest level of nonnursing education". 
Highest level of nursing education by age. Almost half of individuals whose highest level of education is a diploma are older than age 65. Conversely, among those with a baccalaureate, the highest percentage are young nurses (15.4\% < 30 years old). See Table 15 .

\section{TABLE 15}

\section{Highest Level of Nursing Education by Age-RN}

\begin{tabular}{|c|c|c|c|c|c|c|c|c|c|c|}
\hline \multicolumn{11}{|l|}{ Weighted Sample Values } \\
\hline Age cohort & $<30$ & $30-34$ & $35-39$ & $40-44$ & $45-49$ & $50-54$ & $55-59$ & $60-64$ & $\geq 65$ & $n$ \\
\hline Diploma & $0.9 \%$ & $1.1 \%$ & $2.7 \%$ & $2.2 \%$ & $4.1 \%$ & $8.0 \%$ & $13.7 \%$ & $22.6 \%$ & $44.6 \%$ & \\
\hline Associate degree & $6.7 \%$ & $8.5 \%$ & $8.9 \%$ & $9.6 \%$ & $11.8 \%$ & $11.5 \%$ & $13.8 \%$ & $15.0 \%$ & $14.2 \%$ & \\
\hline Baccalaureate degree & $3,281.7$ & $2,618.7$ & $2,092.9$ & $2,070.2$ & $2,471.7$ & $2,131.1$ & $2,335.8$ & $2,275.8$ & $2,018.2$ & $21,296.1$ \\
\hline Master's degree & $4.3 \%$ & $10.6 \%$ & $11.3 \%$ & $10.4 \%$ & $11.4 \%$ & $10.4 \%$ & $12.3 \%$ & $14.7 \%$ & $14.7 \%$ & \\
\hline \multirow[t]{2}{*}{ Doctoral degree-PhD } & 0.3 & 6.0 & 11.8 & 13.7 & 22.3 & 22.2 & 40.6 & 78.8 & 79.7 & 275.2 \\
\hline & $0.1 \%$ & $2.2 \%$ & $4.3 \%$ & $5.0 \%$ & $8.1 \%$ & $8.1 \%$ & $14.7 \%$ & $28.6 \%$ & $28.9 \%$ & \\
\hline \multirow[t]{2}{*}{ Doctoral degree-DNP } & 22.2 & 89.5 & 66.1 & 40.4 & 60.9 & 70.0 & 89.7 & 76.0 & 24.3 & 539.0 \\
\hline & $4.1 \%$ & $16.6 \%$ & $12.3 \%$ & $7.5 \%$ & $11.3 \%$ & $13.0 \%$ & $16.6 \%$ & $14.1 \%$ & $4.5 \%$ & \\
\hline Total & $9.7 \%$ & $10.1 \%$ & $9.3 \%$ & $9.2 \%$ & $11.0 \%$ & $10.4 \%$ & $12.3 \%$ & $13.6 \%$ & $14.4 \%$ & \\
\hline
\end{tabular}

Note. In the 2013 and 2015 survey, a single question "What is your highest level of education?" was asked, with the set of possible responses including both nursing and non-nursing degrees. In 2017, two separate questions were asked, "Highest level of nursing education" and "Highest level of nonnursing education".

\section{Highest level of non-nursing education}

Approximately $40 \%$ of RN respondents reported an additional degree in a non-nursing discipline. Of those, $35.3 \%$ reported an associate level non-nursing degree, $45.8 \%$ reported a baccalaureate level non-nursing degree, and an additional $18.9 \%$ reported a graduate-level non-nursing degree. See Table 16.

\section{TABLE 16}

\section{Highest Level of Non-Nursing Education-RN}

\begin{tabular}{lcc}
\hline Weighted Sample Values & & \multicolumn{2}{c}{$\mathbf{2 0 1 7}(\boldsymbol{n}=\mathbf{1 9 , 9 0 4 . 5 )}$} \\
\hline & $\boldsymbol{n}$ & Percent \\
\hline Associate degree & $7,025.9$ & $35.3 \%$ \\
\hline Baccalaureate degree & $9,115.4$ & $45.8 \%$ \\
\hline Master's degree & $3,150.8$ & $15.8 \%$ \\
\hline Doctoral degree & 612.4 & $3.1 \%$ \\
\hline
\end{tabular}
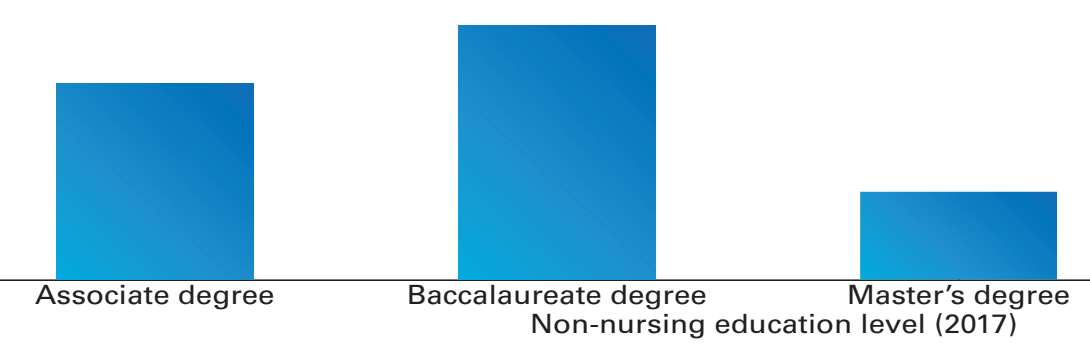

Non-nursing education level (2017)

Note. In the 2013 and 2015 surveys, a single question "What is your highest level of education?" was asked, with the set of possible responses including both nursing and non-nursing degrees. In 2017, two separate questions were asked, "Highest level of nursing education" and "Highest level of nonnursing education". 


\section{Licensure}

Type of license currently held

Respondents were asked to select all of the nursing licenses that they currently hold. Less than $1 \%$ of responding RNs also held an LPN/ LVN license, while $10.0 \%$ held an APRN credential. The percentage of responding RNs who held an APRN credential increased by 1.4 percentage points from 2015 to 2017. See Table 17.

\section{TABLE 17}

\section{Type of License Currently Held-RN}

\begin{tabular}{lcccccc}
\hline Weighted Sample Values & \multicolumn{1}{l}{} & & & & \\
\hline & $\mathbf{2 0 1 3}(\boldsymbol{n = 4 1 , 6 5 8 . 3 )}$ & $\mathbf{2 0 1 5}(\boldsymbol{n = 4 6 , 0 4 7 . 8 )}$ & $\mathbf{2 0 1 7}(\boldsymbol{n = 4 8 , 1 2 8 . 0 )}$ \\
\hline RN & $\boldsymbol{n}$ & Percent & $\boldsymbol{n}$ & Percent & $\boldsymbol{n}$ & Percent \\
\hline LPN & $39,521.6$ & $94.9 \%$ & $43,730.2$ & $95.0 \%$ & $45,971.3$ & $95.5 \%$ \\
\hline APRN & 215.9 & $0.5 \%$ & 330.8 & $0.7 \%$ & 386.2 & $0.8 \%$ \\
\hline
\end{tabular}

\begin{tabular}{|c|c|c|c|c|c|c|}
\hline \multicolumn{7}{|c|}{ Estimated Population Values } \\
\hline & \multicolumn{2}{|c|}{2013} & \multicolumn{2}{|c|}{2015} & \multicolumn{2}{|c|}{2017} \\
\hline & Freq & Percent & Freq & Percent & Freq & Percent \\
\hline LPN & 17,845 & $0.5 \%$ & 26,534 & $0.7 \%$ & 31,328 & $0.8 \%$ \\
\hline APRN & 251,788 & $7.3 \%$ & 318,870 & $8.6 \%$ & 388,461 & $10.0 \%$ \\
\hline
\end{tabular}

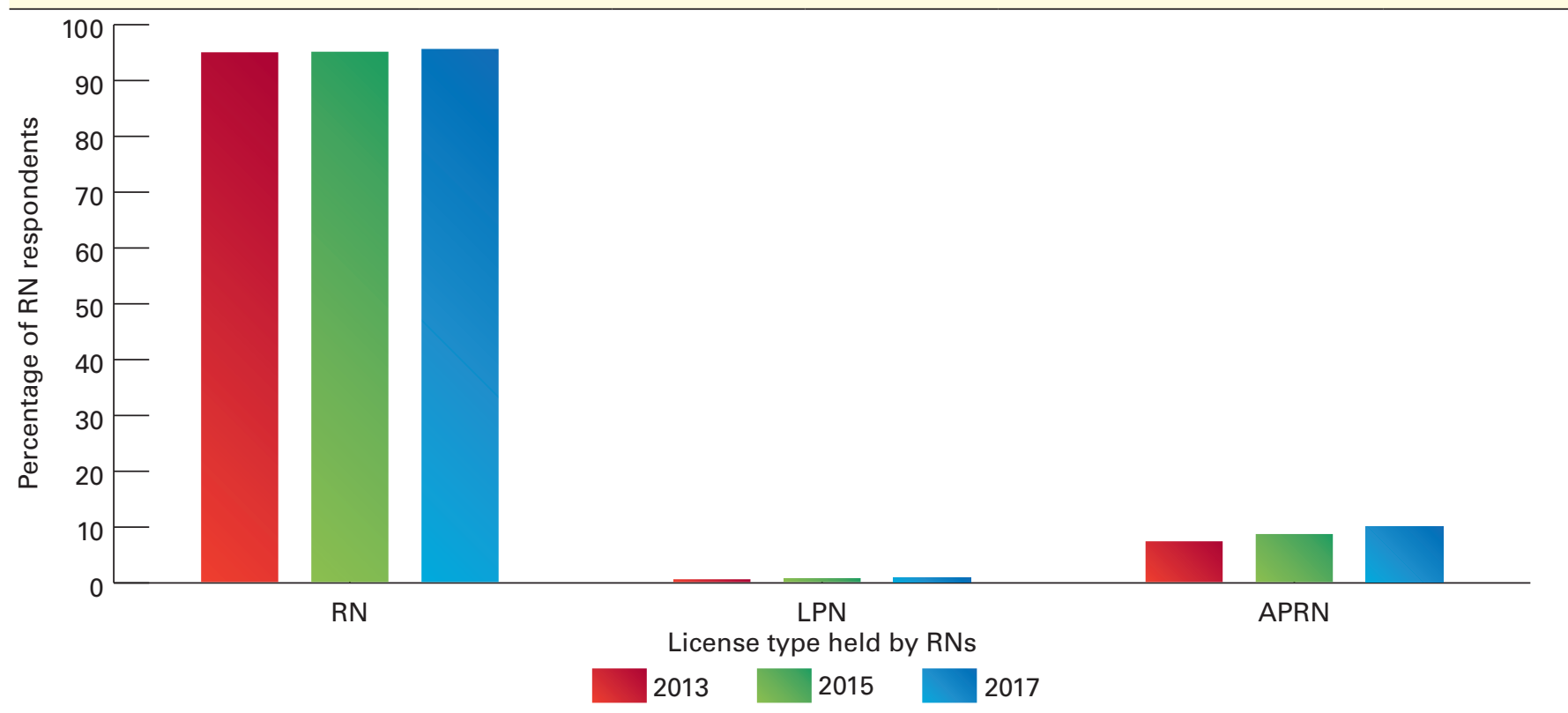




\section{Number of years licensed}

RN respondents to the 2017 survey were licensed for a median 21 years. Nearly one-third of respondents (32.9\%) were licensed for 10 years or less. An additional 19.7\% were licensed between 11 and 20 years. These proportions are consistent with those from $2015,33.5 \%$ and $19.5 \%$, respectively. See Table 18 .

\section{TABLE 18}

\section{Number of Years Licensed-RN}

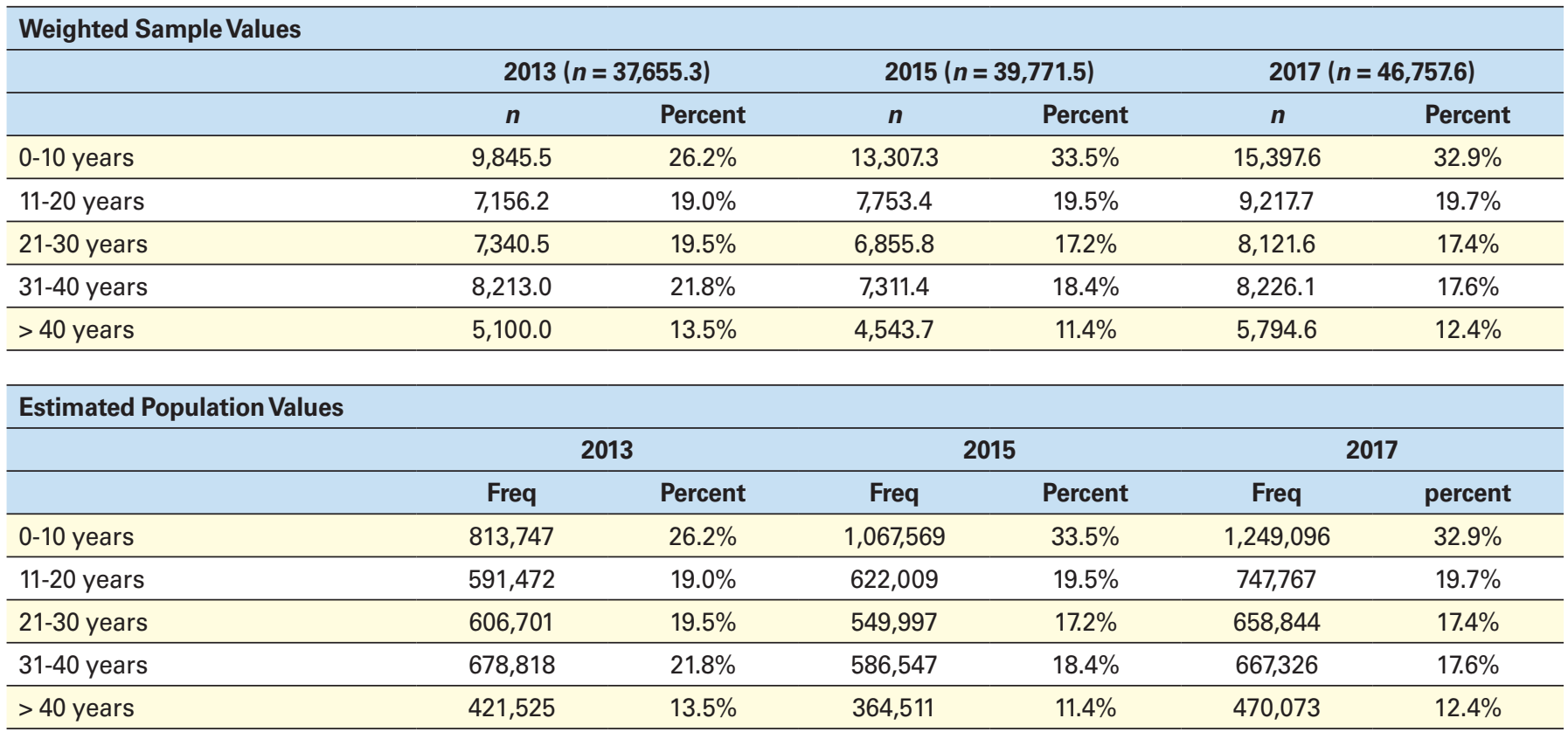

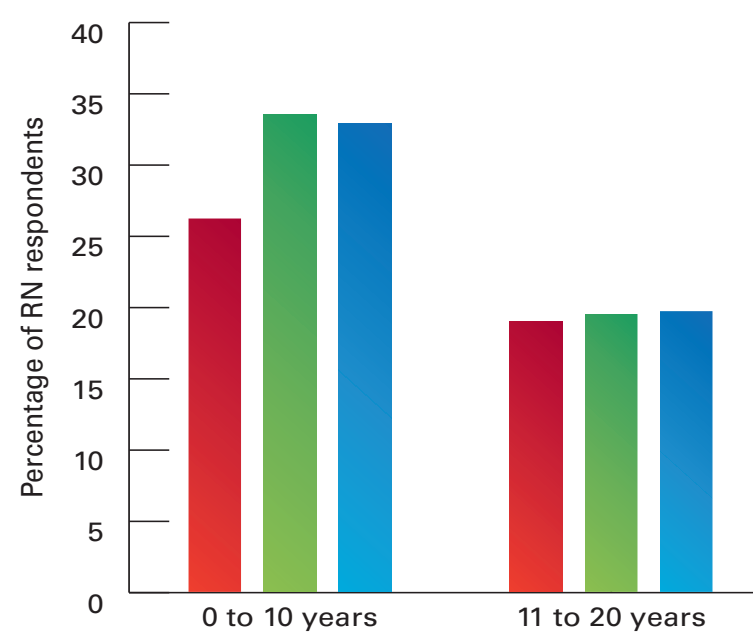

Number of years licensed

\section{$2013 \quad 2015 \quad 2017$}


In 2017, respondents were asked whether they were licensed in the United States initially. This question differs slightly from 2013 and 2015, when respondents were asked in what country they were initially licensed. In 2017, 95.1\% of respondents were initially licensed in the United States. This is consistent with results from 2015 (94.6\%) and 2013 (95.0\%). See Table 19.

\section{TABLE 19}

\section{Initially Licensed in the United States-RN}

\begin{tabular}{lcccccc}
\hline Weighted Sample Values & \multicolumn{1}{c}{} & & & \\
\hline & $\mathbf{2 0 1 3}$ & $(\boldsymbol{n = 4 0 , 3 9 3 . 1 )}$ & $\mathbf{2 0 1 5}(\boldsymbol{n = 4 5 , 3 0 4 . 6 )}$ & \multicolumn{2}{c}{$\mathbf{2 0 1 7}(\boldsymbol{n}=\mathbf{4 8 , 0 9 5 . 0 )}$} \\
\hline No & $\boldsymbol{n}$ & Percent & $\boldsymbol{n}$ & Percent & $\boldsymbol{n}$ & Percent \\
\hline Yes & $2,027.7$ & $5.0 \%$ & $2,444.2$ & $5.4 \%$ & $2,380.3$ & $4.9 \%$ \\
\hline
\end{tabular}

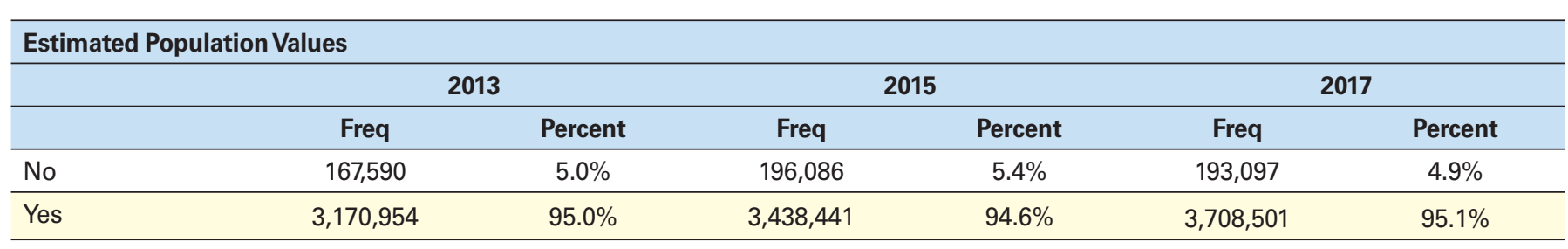

Note. In the 2013 and 2015 surveys, respondents were asked "In what country were you initially licensed as an RN or LPN?" In 2017, respondents were asked whether they were initially licensed as an RN or LPN in the United States.

\section{Credentialed to practice as an APRN}

Respondents were asked whether they were credentialed in their state as an APRN enabling them to practice in any of the 4 APRN roles, including NP, CNS, CRNA, or CNM. A large number of respondents (88.0\%) indicated they were not credentialed as an APRN. This represents a lower proportion than in 2015 or 2013 (89.7\% and $90.8 \%$ respectively). In $2017,8.5 \%$ reported being credentialed as a nurse practitioner compared to $7.2 \%$ in 2015. See Table 20.

\section{TABLE 20}

\section{Credentialed to Practice as an APRN}

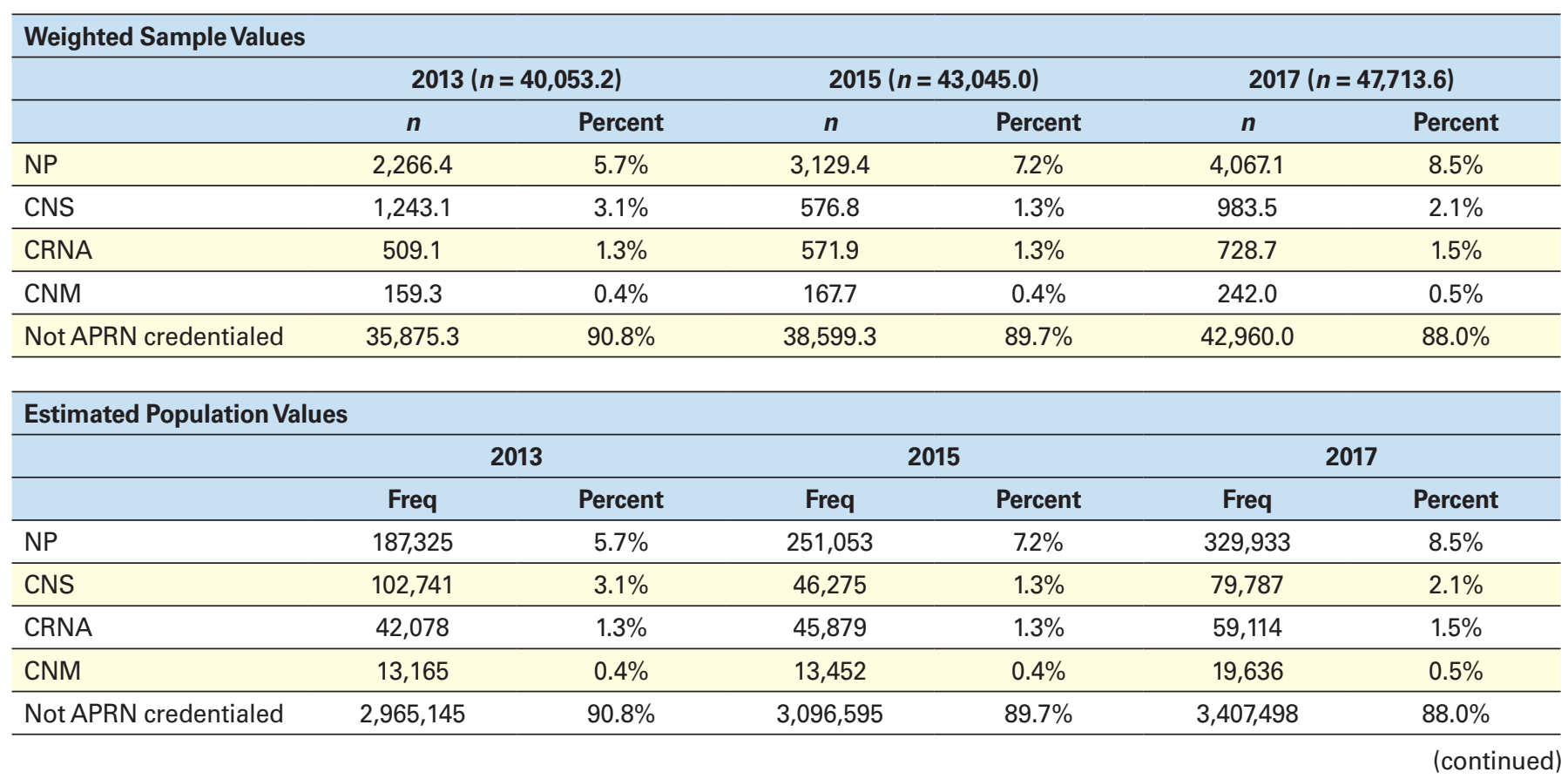




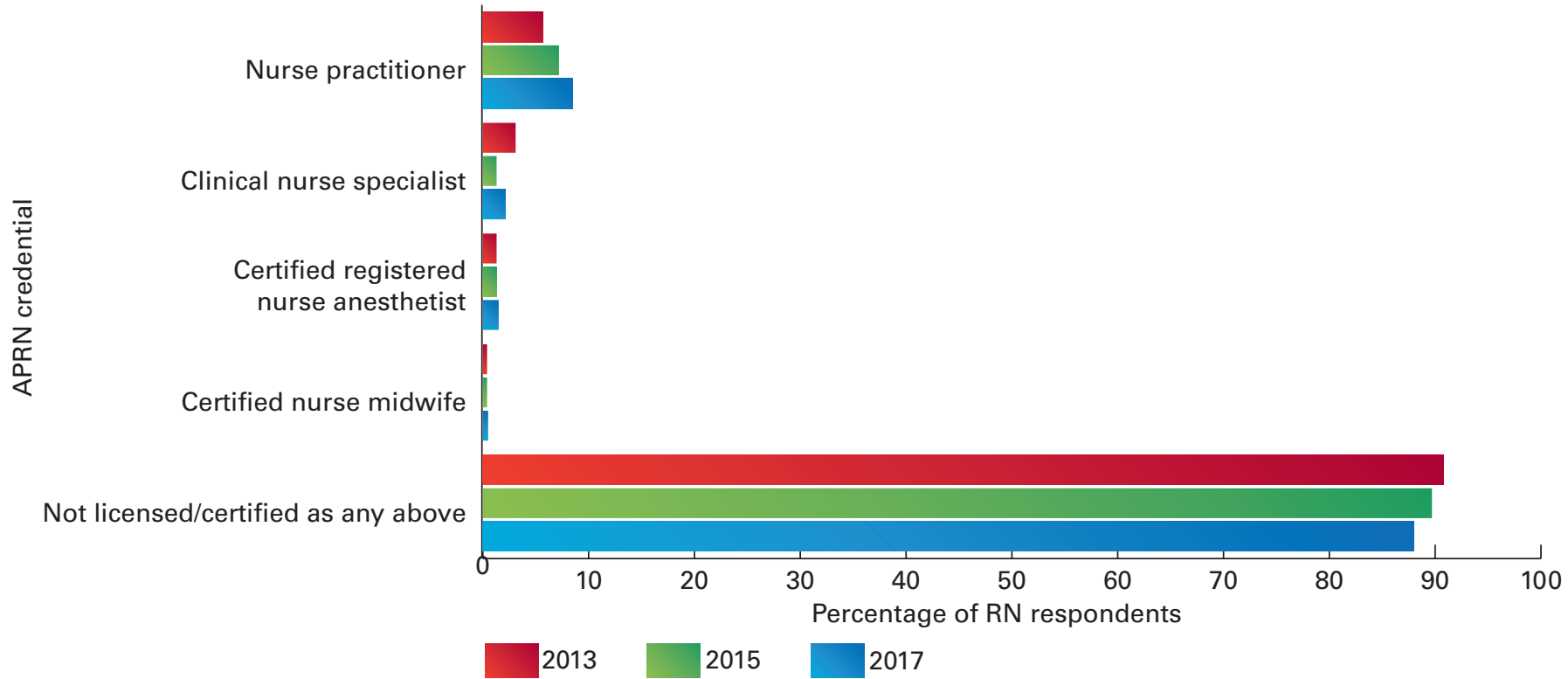

Note. Respondents were asked to select all that apply. NP = nurse practitioner; CNS = clinical nurse specialist; CRNA = certified registered nurse anesthetist; $\mathrm{CNM}=$ certified nurse midwife.

\section{Employment}

\section{Employment status}

The vast majority of responding RNs (84.6\%) were actively employed in nursing and $65.4 \%$ were employed in nursing full time. This represents a continued increase in the proportion of RNs working full time from 2015 (62.9\%) and 2013 (60.4\%). See Table 21.

\section{TABLE 21}

\section{Employment Status-RN}

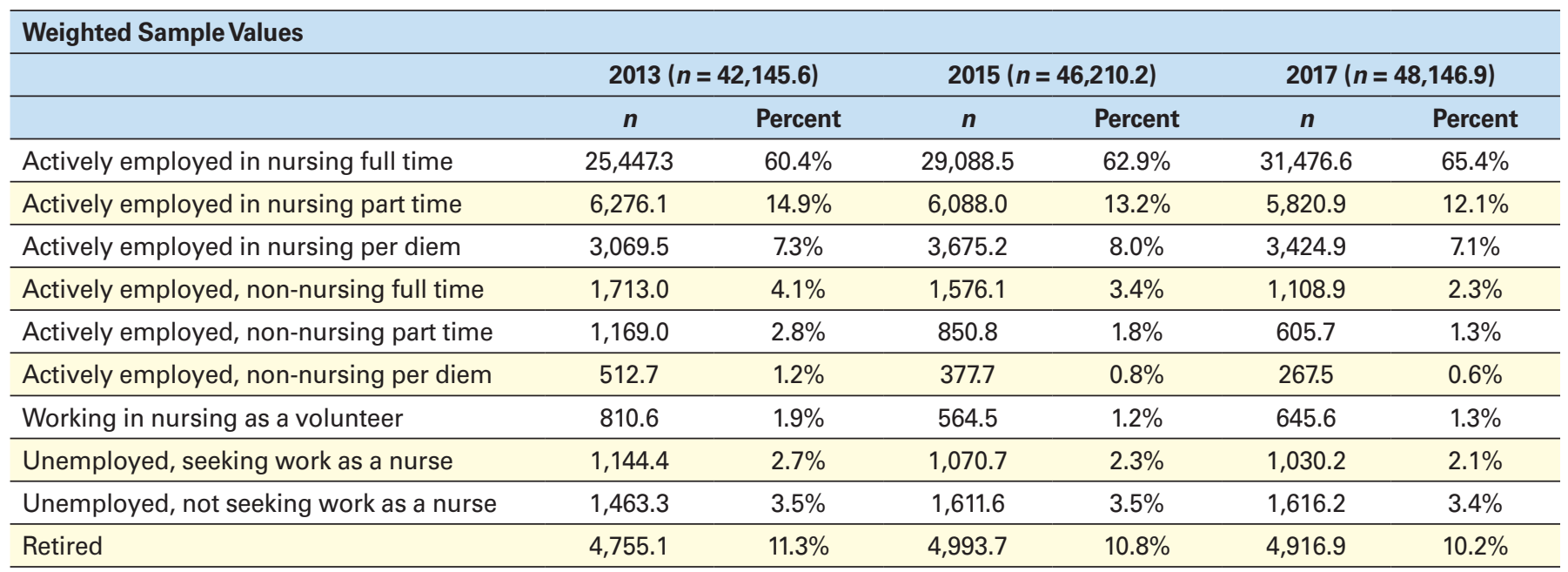

\begin{tabular}{lcccccc}
\hline Estimated Population Values & & & & & & \\
& \multicolumn{2}{c}{$\mathbf{2 0 1 3}$} & & $\mathbf{2 0 1 5}$ & & 2017 \\
\hline & Freq & Percent & Freq & Percent & Freq & Percent \\
\hline Actively employed in nursing full time & $2,103,254$ & $60.4 \%$ & $2,333,606$ & $62.9 \%$ & $2,553,467$ & $65.4 \%$ \\
\hline Actively employed in nursing part time & 518,727 & $14.9 \%$ & 488,405 & $13.2 \%$ & 472,204 & $12.1 \%$ \\
\hline Actively employed in nursing per diem & 253,696 & $7.3 \%$ & 294,837 & $8.0 \%$ & 277,834 & $7.1 \%$ \\
\hline Actively employed, non-nursing full time & 141,583 & $4.1 \%$ & 126,445 & $3.4 \%$ & 89,956 & $2.3 \%$ \\
\hline
\end{tabular}




\section{Employment Status - RN (continued)}

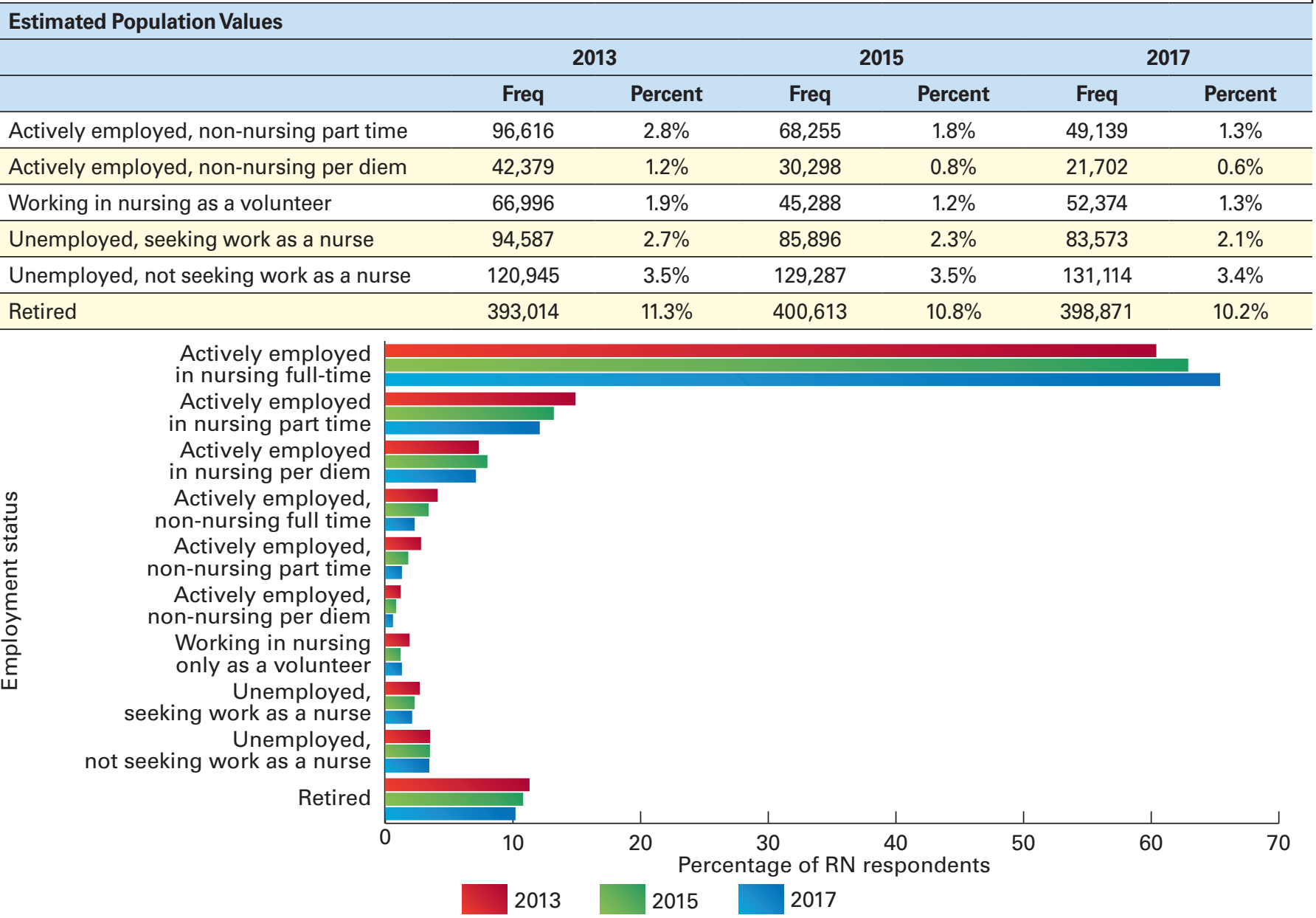

Note. Respondents were asked to select all that apply.

\section{Reasons for being unemployed}

Respondents were asked to select all the reasons for being unemployed. Taking care of home and family was the most frequently selected reason for being unemployed by $47.8 \%$ of respondents. The proportion of RNs who indicated that they were unemployed because they experienced difficulty in finding a nursing position were similar in 2015 (15.5\%) and 2017 (15.4\%). See Table 22.

TABLE 22

\section{Reasons for Being Unemployed-RN}

\begin{tabular}{|c|c|c|c|c|c|c|}
\hline \multicolumn{7}{|l|}{ Weighted Sample Values } \\
\hline & \multicolumn{2}{|c|}{$2013(n=2,549.0)$} & \multicolumn{2}{|c|}{$2015(n=2,272.4)$} & \multicolumn{2}{|c|}{$2017(n=2,567.2)$} \\
\hline & $n$ & Percent & $n$ & Percent & $n$ & Percent \\
\hline Disabled & 486.2 & $19.1 \%$ & 298.5 & $13.1 \%$ & 347.6 & $13.5 \%$ \\
\hline Inadequate salary & 58.4 & $2.3 \%$ & 48.2 & $2.1 \%$ & 73.7 & $2.9 \%$ \\
\hline Difficulty in finding a nursing position & 672.1 & $26.4 \%$ & 352.0 & $15.5 \%$ & 395.0 & $15.4 \%$ \\
\hline Other & 774.8 & $30.4 \%$ & 557.4 & $24.5 \%$ & 758.9 & $29.6 \%$ \\
\hline
\end{tabular}


Taking care of home and family

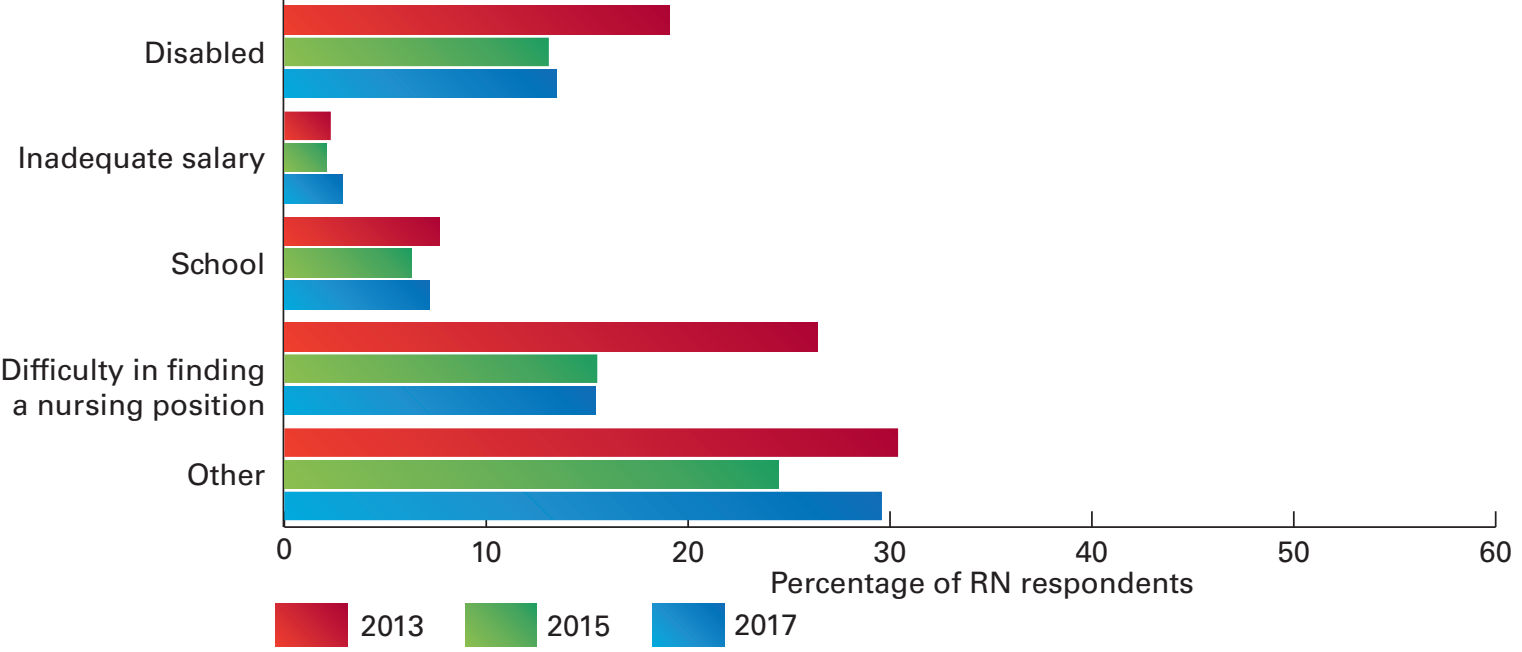

Note. Respondents were asked to select all that apply.

\section{Number of positions currently held}

Respondents were asked to identify the number of positions in which they were currently employed as a nurse. The majority of respondents (83.3\%) reported holding just one position as a nurse, which represents a 1.6 percentage point decrease when compared to 2015. The percentage of nurses who reported working in two positions increased from $12.8 \%$ in 2015 to $13.9 \%$ in 2017 . The percentage of respondents who indicated that they held three or more positions in nursing also increased slightly from $2.4 \%$ in 2015 to $2.8 \%$ in 2017. See Table 23.

\section{TABLE 23}

\section{Number of Positions Currently Held-RN}

\begin{tabular}{|c|c|c|c|c|c|c|}
\hline \multicolumn{7}{|c|}{ Weighted Sample Values } \\
\hline & \multicolumn{2}{|c|}{$2013(n=33,264.5)$} & \multicolumn{2}{|c|}{$2015(n=37,114.2)$} & \multicolumn{2}{|c|}{$2017(n=39,414.3)$} \\
\hline & $n$ & Percent & $n$ & Percent & $n$ & Percent \\
\hline 2 & $4,434.6$ & $13.3 \%$ & $4,744.0$ & $12.8 \%$ & $5,496.7$ & $13.9 \%$ \\
\hline 3 or more & 760.8 & $2.3 \%$ & 870.8 & $2.4 \%$ & $1,090.5$ & $2.8 \%$ \\
\hline
\end{tabular}

\begin{tabular}{|c|c|c|c|c|c|c|}
\hline \multicolumn{7}{|c|}{ Estimated Population Values } \\
\hline & \multicolumn{2}{|c|}{2013} & \multicolumn{2}{|c|}{2015} & \multicolumn{2}{|c|}{2017} \\
\hline & Freq & Percent & Freq & Percent & Freq & Percent \\
\hline 2 & 366,522 & $13.3 \%$ & 380,585 & $12.8 \%$ & 445,905 & $13.9 \%$ \\
\hline 3 or more & 62,883 & $2.3 \%$ & 69,861 & $2.4 \%$ & 88,463 & $2.8 \%$ \\
\hline
\end{tabular}

Note. Survey participants were asked to answer this question only if they were actively employed in nursing. 
Hours worked categories were recoded for the 2017 report. Over half (58.6\%) of responding RNs reported working 32 to 40 hours in a typical week in all positions. This is consistent with the results from the 2015 survey (58.4\%) and only 2.1 percentage points higher than $2013(56.5 \%)$. The second most frequently reported category was 41 to 50 hours (15.8\%). This represents a slight decrease from $2015(16.4 \%)$ and $2013(16.2 \%)$. See Table 24.

TABLE 24

\section{Number of Hours Worked During a Typical Week in All Nursing Positions-RN}

\begin{tabular}{|c|c|c|c|c|c|c|}
\hline \multicolumn{7}{|c|}{ Weighted Sample Values } \\
\hline & \multicolumn{2}{|c|}{$2013(n=32,645.8)$} & \multicolumn{2}{|c|}{$2015(n=36,327.6)$} & \multicolumn{2}{|c|}{$2017(n=39,293.3)$} \\
\hline & $n$ & Percent & $n$ & Percent & $n$ & Percent \\
\hline $16-23$ & $1,695.4$ & $5.2 \%$ & $1,655.7$ & $4.6 \%$ & $1,728.1$ & $4.4 \%$ \\
\hline $24-31$ & 3,397.7 & $10.4 \%$ & $3,536.9$ & $9.8 \%$ & $3,765.0$ & $9.6 \%$ \\
\hline $41-50$ & $5,289.3$ & $16.2 \%$ & $5,957.3$ & $16.4 \%$ & $6,198.0$ & $15.8 \%$ \\
\hline $51-60$ & $1,369.5$ & $4.2 \%$ & $1,636.9$ & $4.5 \%$ & $1,851.3$ & $4.7 \%$ \\
\hline 61 or more & 651.0 & $2.0 \%$ & 578.8 & $1.6 \%$ & 834.7 & $2.1 \%$ \\
\hline
\end{tabular}

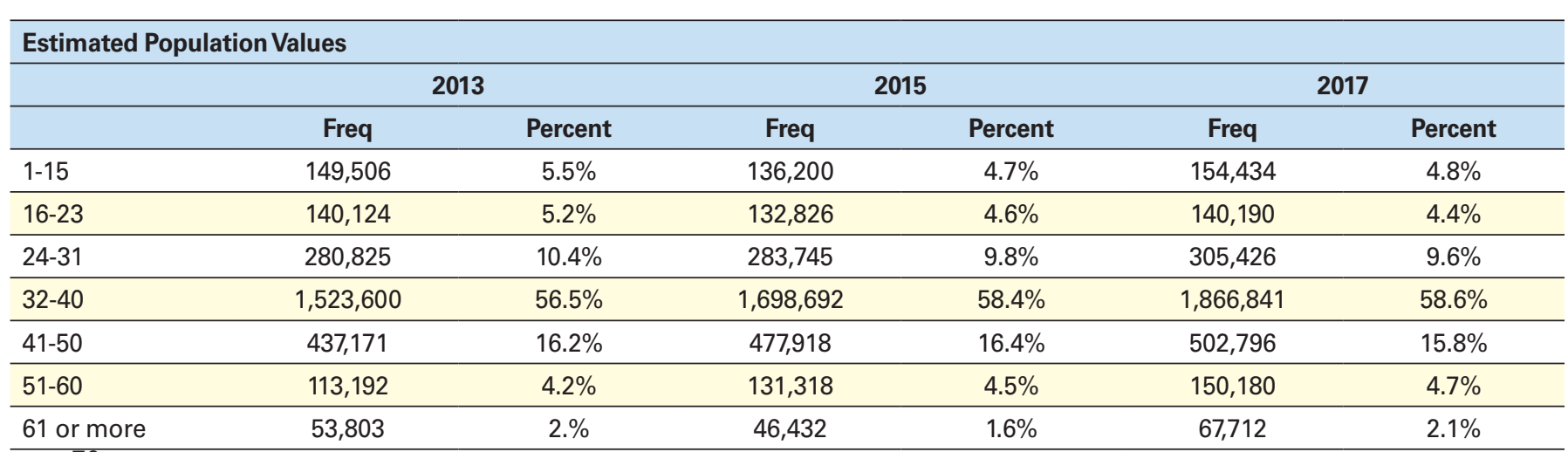

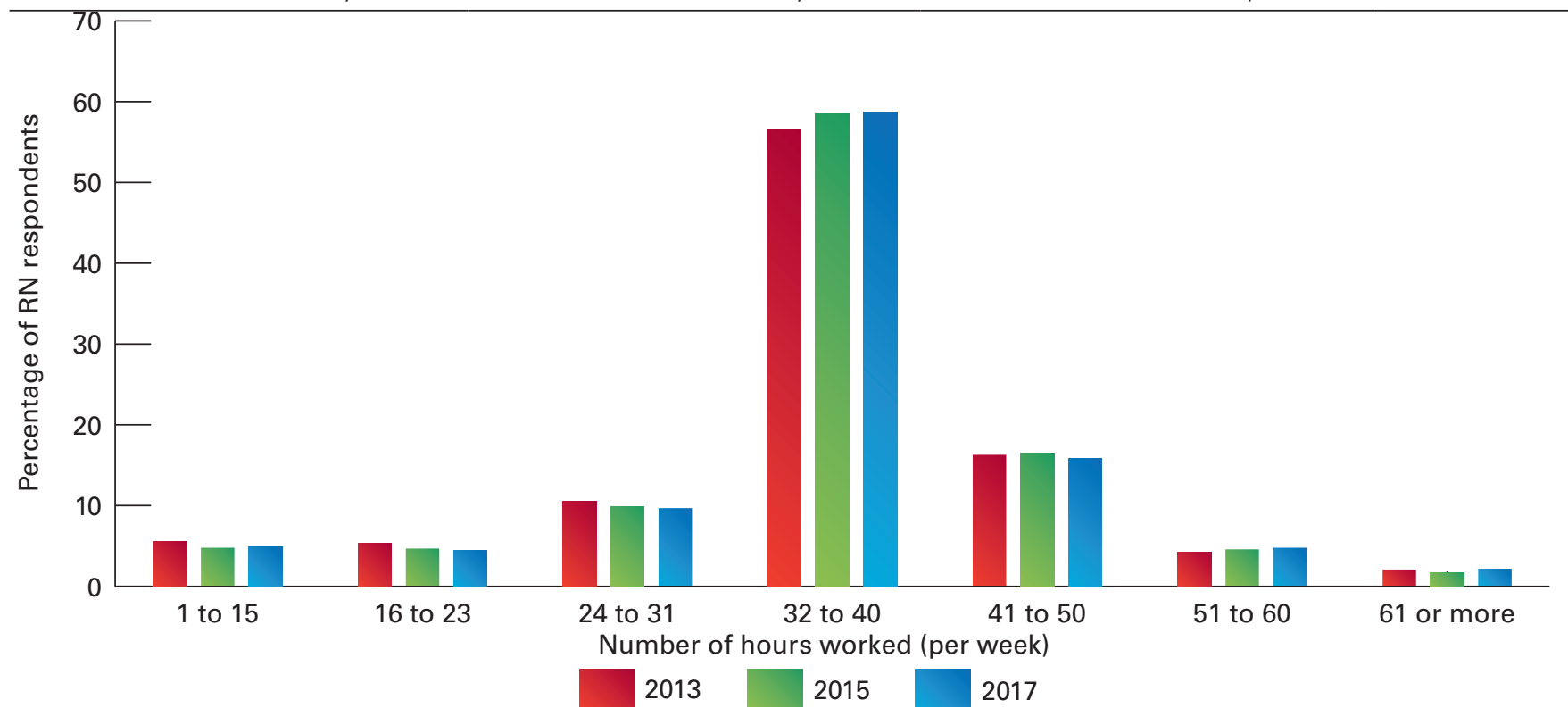

Note. Survey participants were asked to answer this question only if they were actively employed in nursing. 


\section{Primary nursing practice position setting}

In the 2017 survey, response options related to primary and secondary practice setting were slightly modified. Hospice and dialysis center were added as new categories. Of those who responded to the question, $55.7 \%$ indicated that a hospital was their primary nursing practice. This represents an increase of 1.3 percentage points from 2015 but still fewer than the $56.5 \%$ of respondents that selected this setting in 2013. Ambulatory care setting was the second most frequently selected setting by $9.4 \%$ of RNs, followed by nursing home/ extended care setting selected by $4.8 \%$, and home health selected by $4.3 \%$. Each of the remaining settings were selected by fewer than $3 \%$ of the responding nurses. Additionally, $9.8 \%$ of respondents selected other. See Table 25.

TABLE 25

\section{Primary Nursing Practice Position Setting-RN}

\begin{tabular}{|c|c|c|c|c|c|c|}
\hline \multicolumn{7}{|l|}{ Weighted Sample Values } \\
\hline & \multicolumn{2}{|c|}{$2013(n=34,238.0)$} & \multicolumn{2}{|c|}{$2015(n=37,372.1)$} & \multicolumn{2}{|c|}{$2017(n=38,870.1)$} \\
\hline & $n$ & percent & $n$ & percent & $n$ & percent \\
\hline Nursing home/extended care & $2,210.8$ & $6.5 \%$ & $1,807.2$ & $4.8 \%$ & $1,859.7$ & $4.8 \%$ \\
\hline Assisted living facility & --- & --- & 233.3 & $0.6 \%$ & 211.2 & $0.5 \%$ \\
\hline Correctional facility & 229.0 & $0.7 \%$ & 259.6 & $0.7 \%$ & 294.8 & $0.8 \%$ \\
\hline School of nursing & $1,011.9$ & $3.0 \%$ & $1,357.0$ & $3.6 \%$ & $1,028.9$ & $2.6 \%$ \\
\hline Public health & 609.2 & $1.8 \%$ & 595.4 & $1.6 \%$ & 539.3 & $1.4 \%$ \\
\hline Dialysis center & --- & --- & --- & --- & 493.6 & $1.3 \%$ \\
\hline Community health & 739.9 & $2.2 \%$ & 786.9 & $2.1 \%$ & 780.8 & $2.0 \%$ \\
\hline Insurance claims/benefits & 477.2 & $1.4 \%$ & 673.7 & $1.8 \%$ & 694.1 & $1.8 \%$ \\
\hline Policy/planning/regulatory/licensing agency & 152.3 & $0.4 \%$ & 148.7 & $0.4 \%$ & 86.9 & $0.2 \%$ \\
\hline Other & $3,042.1$ & $8.9 \%$ & $3,366.3$ & $9.0 \%$ & $3,823.6$ & $9.8 \%$ \\
\hline
\end{tabular}

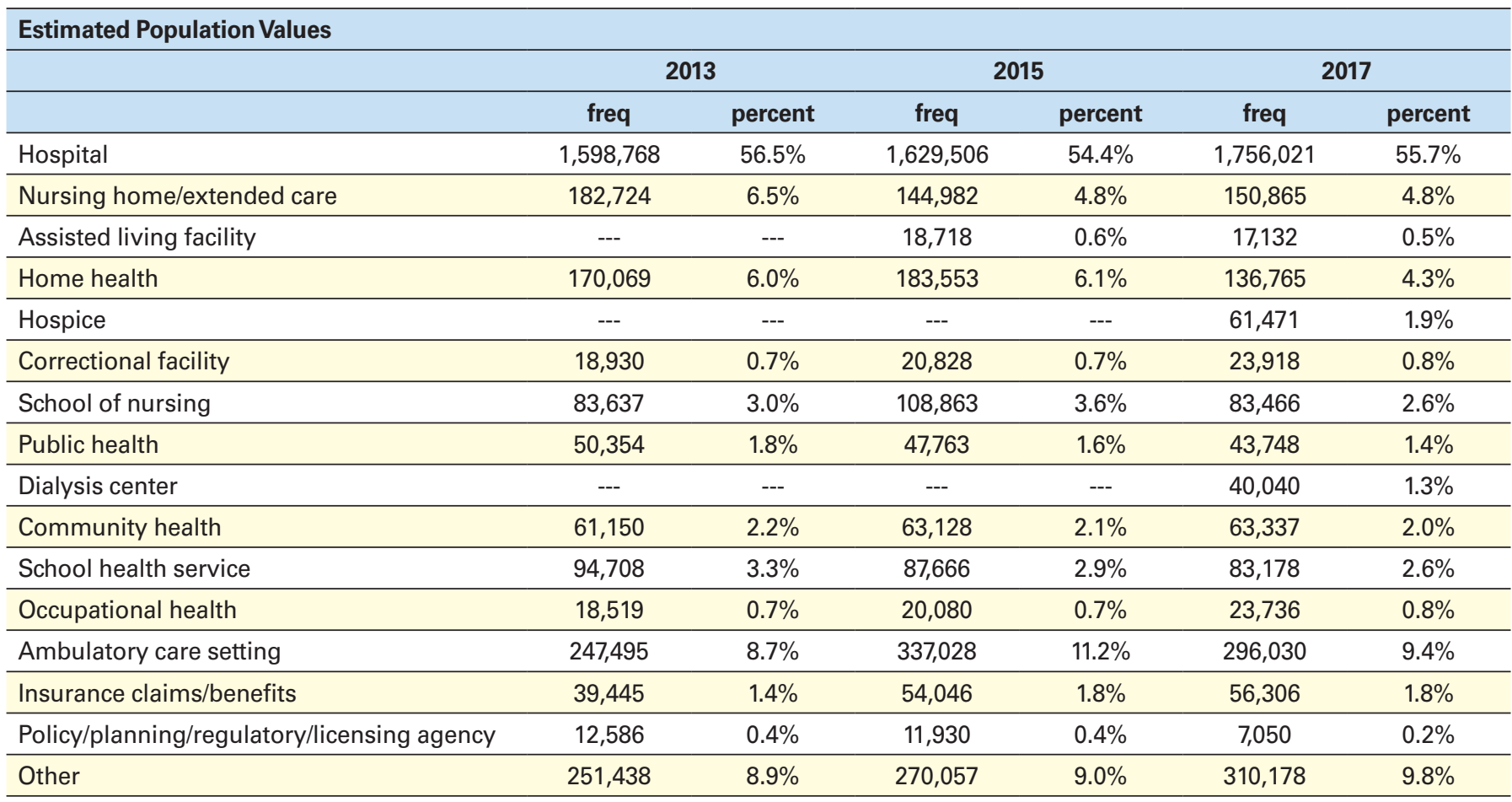

(continued) 


\section{Primary Nursing Practice Position Setting-RN (continued)}

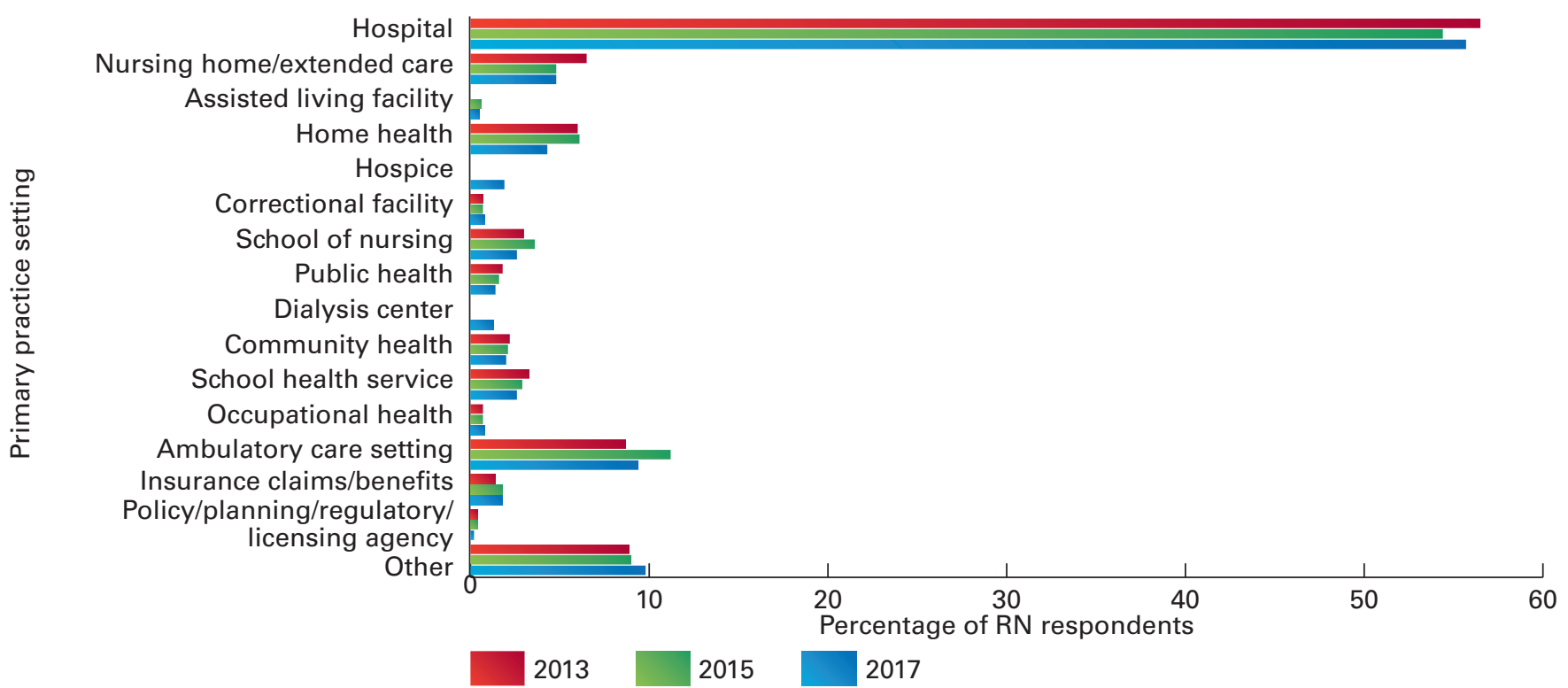

Note. Survey participants were asked to answer this question only if they were actively employed in nursing. Salary includes overtime and bonuses, but does not include sign-on bonuses.

\section{Primary nursing position title}

As in 2015, 58.0\% of 2017 respondents indicated that the title that most closely corresponds to their primary nursing position was that of staff nurse. Advanced practice registered nurse was the second most frequently selected position by $10.1 \%$ of respondents. This represents an increase from $8.1 \%$ in 2015. Between 2013 and 2017, there was a 3.0 percentage point decrease in respondents who indicated that their primary practice position was nurse manager. RNs selecting case manager as their primary position $(6.4 \%)$ was in alignment with what was reported in $2015(6.7 \%)$. The percentage of respondents who selected nurse faculty/educator increased slightly from $3.8 \%$ in 2015 to $4.0 \%$ in 2017. Each of the remaining positions were selected by less than $2 \%$ of the responding nurses. Additionally, $9.1 \%$ of respondents selected other-health related and $0.4 \%$ selected other-non-health related. See Table 26.

\section{TABLE 26}

\section{Primary Nursing Position Title-RN}

\begin{tabular}{|c|c|c|c|c|c|c|}
\hline \multicolumn{7}{|l|}{ Weighted Sample Values } \\
\hline & \multicolumn{2}{|c|}{$2013(n=34,356.6)$} & \multicolumn{2}{|c|}{$2015(n=37,711.1)$} & \multicolumn{2}{|c|}{$2017(n=39,063.1)$} \\
\hline & $n$ & percent & $n$ & percent & $n$ & percent \\
\hline Nurse researcher & 251.3 & $0.7 \%$ & 247.2 & $0.7 \%$ & 235.9 & $0.6 \%$ \\
\hline Nurse executive & 834.4 & $2.4 \%$ & 881.4 & $2.3 \%$ & 725.3 & $1.9 \%$ \\
\hline Nurse faculty/educator & $1,105.4$ & $3.2 \%$ & $1,422.2$ & $3.8 \%$ & $1,558.2$ & $4.0 \%$ \\
\hline Advanced practice registered nurse & $2,531.4$ & $7.4 \%$ & $3,069.1$ & $8.1 \%$ & $3,946.1$ & $10.1 \%$ \\
\hline Staff nurse & $21,902.1$ & $63.7 \%$ & $21,920.7$ & $58.1 \%$ & $22,673.0$ & $58.0 \%$ \\
\hline Case manager & --- & --- & $2,524.8$ & $6.7 \%$ & $2,519.2$ & $6.4 \%$ \\
\hline Other-health related & $3,068.9$ & $8.9 \%$ & $3,685.1$ & $9.8 \%$ & $3,561.9$ & $9.1 \%$ \\
\hline
\end{tabular}




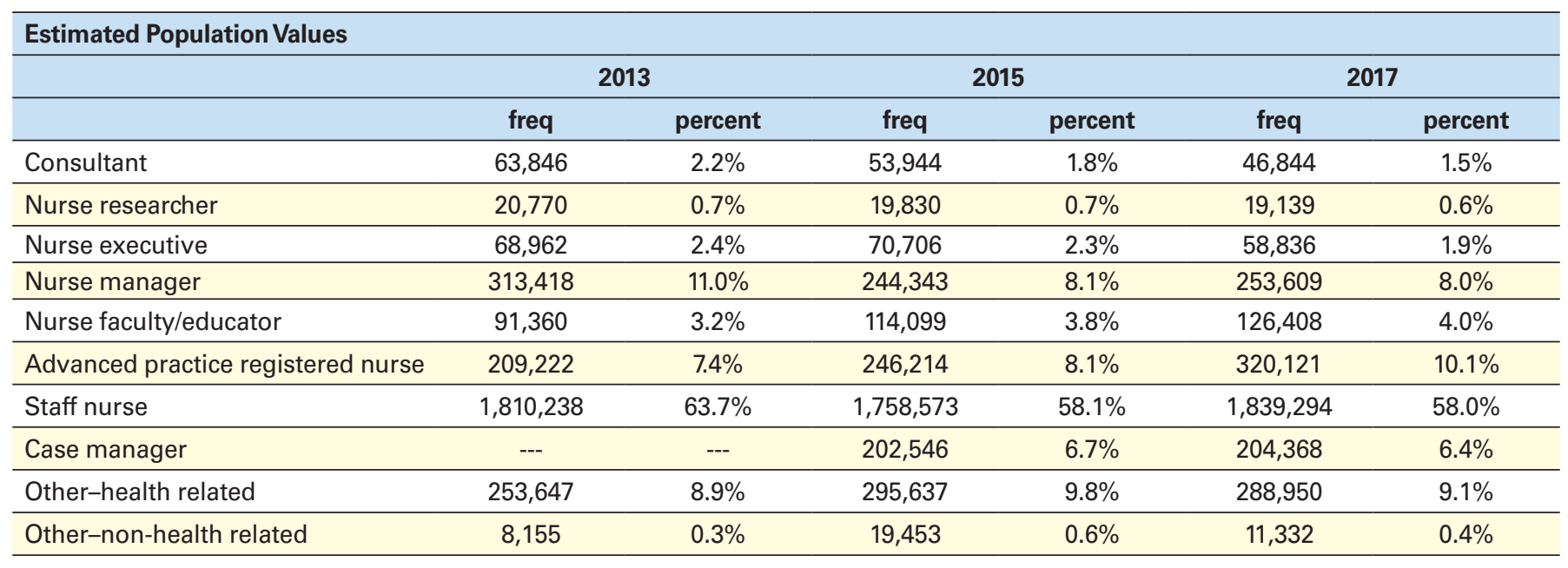

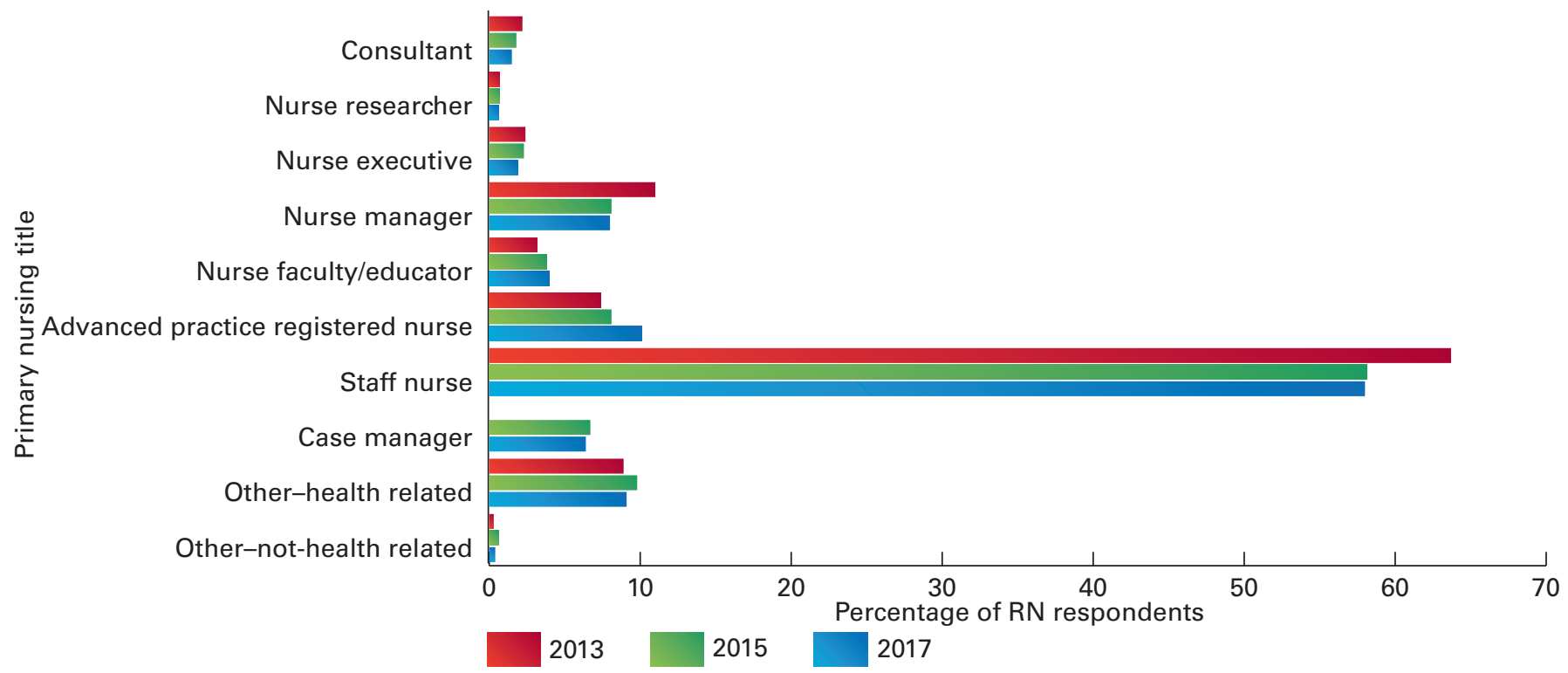

Note. Survey participants were asked to answer this question only if they were actively employed in nursing. Salary includes overtime and bonuses, but does not include sign-on bonuses.

\section{Primary nursing position specialty}

Response options related to primary and secondary practice specialty were also modified. Adult health and family health were separated into two categories, cardiology was added as a category, and other was separated into other-clinical and other-non-clinical. Primary care, genetics, informatics, neurology/neurosurgical, orthopedic, radiology, urology, and telehealth were all removed from the list of categories.

In the current study, $14 \%$ of RNs reported their primary practice specialty was acute care/critical care compared to $11.4 \%$ in 2015 . The second most frequently selected specialty was medical-surgical; $8.5 \%$ of $\mathrm{RN}$ respondents selected this specialty in 2017 compared to $10.1 \%$ in 2015 . Perioperative was the third most frequently selected specialty by $5.8 \%$ of 2017 respondents. RNs selecting other specialties $(14.1 \%)$ represented a decrease from $17.0 \%$ in 2015 . See Table 27. 


\section{TABLE 27}

\section{Primary Nursing Position Specialty-RN}

\begin{tabular}{|c|c|c|c|c|c|c|}
\hline \multicolumn{7}{|l|}{ Weighted Sample Values } \\
\hline & \multicolumn{2}{|c|}{$2013(n=33,516.5)$} & \multicolumn{2}{|c|}{$2015(n=36,424.1)$} & \multicolumn{2}{|c|}{$2017(n=37,484.3)$} \\
\hline & $n$ & Percent & $n$ & Percent & $n$ & Percent \\
\hline Adult health & 871.9 & $2.6 \%$ & 756.1 & $2.1 \%$ & $1,447.1$ & $3.9 \%$ \\
\hline Family health & --- & --- & --- & --- & $1,243.4$ & $3.3 \%$ \\
\hline Cardiology & --- & --- & --- & --- & $1,291.0$ & $3.4 \%$ \\
\hline Community & 335.0 & $1.0 \%$ & 356.7 & $1.0 \%$ & 386.6 & $1.0 \%$ \\
\hline Geriatric/gerontology & $1,988.8$ & $5.9 \%$ & $1,754.7$ & $4.8 \%$ & $1,918.5$ & $5.1 \%$ \\
\hline Home health & $1,515.3$ & $4.5 \%$ & $1,604.0$ & $4.4 \%$ & $1,360.1$ & $3.6 \%$ \\
\hline Occupational health & 333.4 & $1.0 \%$ & 280.7 & $0.8 \%$ & 339.8 & $0.9 \%$ \\
\hline Oncology & 952.7 & $2.8 \%$ & $1,044.0$ & $2.9 \%$ & $1,046.9$ & $2.8 \%$ \\
\hline Palliative care/hospice & 498.9 & $1.5 \%$ & 529.1 & $1.5 \%$ & 643.3 & $1.7 \%$ \\
\hline Pediatrics & $1,995.8$ & $6.0 \%$ & $1,570.3$ & $4.3 \%$ & $1,774.1$ & $4.7 \%$ \\
\hline Neonatal & --- & --- & 808.4 & $2.2 \%$ & 809.5 & $2.2 \%$ \\
\hline Perioperative & --- & --- & $2,195.7$ & $6.0 \%$ & $2,187.7$ & $5.8 \%$ \\
\hline Public health & 510.6 & $1.5 \%$ & 466.0 & $1.3 \%$ & 472.3 & $1.3 \%$ \\
\hline Psychiatric/mental health/substance abuse & $1,340.5$ & $4.0 \%$ & $1,418.4$ & $3.9 \%$ & $1,534.1$ & $4.1 \%$ \\
\hline Other-non-clinical specialties & --- & -- & -- & --- & 775.1 & $2.1 \%$ \\
\hline Other & $6,568.4$ & $19.6 \%$ & $6,200.8$ & $17.0 \%$ & -- & --- \\
\hline Primary care & $1,340.5$ & $2.6 \%$ & $1,092.5$ & $3.0 \%$ & -- & -- \\
\hline Genetics & --- & --- & 40.6 & $0.1 \%$ & --- & --- \\
\hline Informatics & -- & -- & 318.2 & $0.9 \%$ & -- & -- \\
\hline Neurology/neurosurgical & --- & -- & 337.1 & $0.9 \%$ & --- & --- \\
\hline Orthopedic & -- & -- & 436.1 & $1.2 \%$ & --- & -- \\
\hline Radiology & -- & -- & 191.2 & $0.5 \%$ & -- & --- \\
\hline Urology & -- & -- & 87.5 & $0.2 \%$ & -- & --- \\
\hline Telehealth & 288.5 & $1.2 \%$ & --- & --- & --- & --- \\
\hline
\end{tabular}




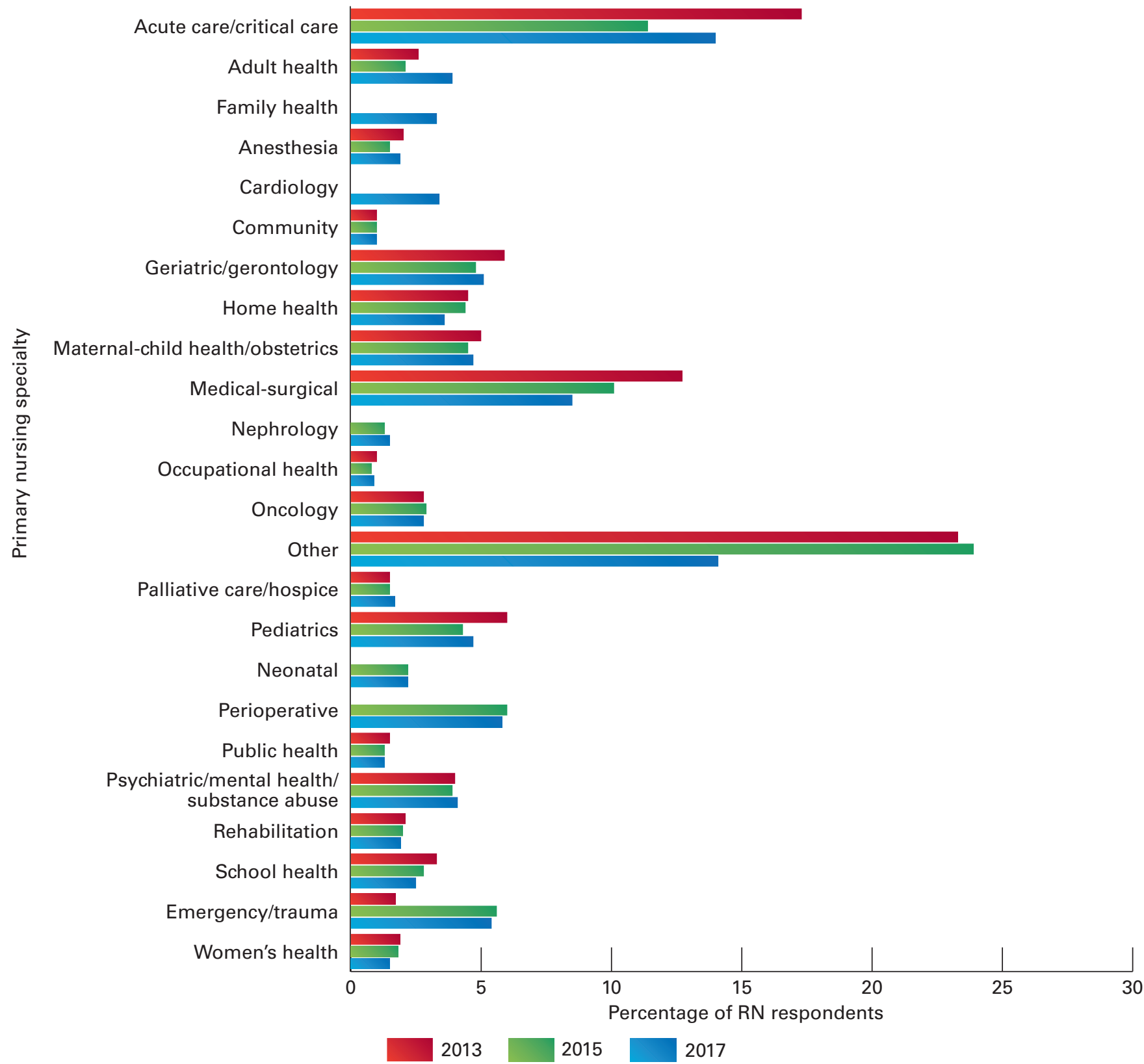

Note. Survey participants were asked to answer this question only if they were actively employed in nursing.

\section{Secondary nursing practice position setting}

A little more than a third of $\mathrm{RN}$ respondents (36.0\%) identified their secondary nursing position setting as being in a hospital. This has consistently been the most frequently selected secondary practice position setting over the past three surveys. The second most frequently selected setting for secondary nursing positions was ambulatory care (9.1\%), followed by home health (9.0\%) and nursing home/extended care $(7.4 \%)$. This is slightly different than in 2015 , when the top three most frequently selected categories for this question were hospitals (33.5\%), home health (11.8\%), and school of nursing (10.7\%), and similar to the top settings reported for primary practice positions. See Table 28. 


\section{TABLE 28}

\section{Secondary Nursing Practice Position Setting-RN}

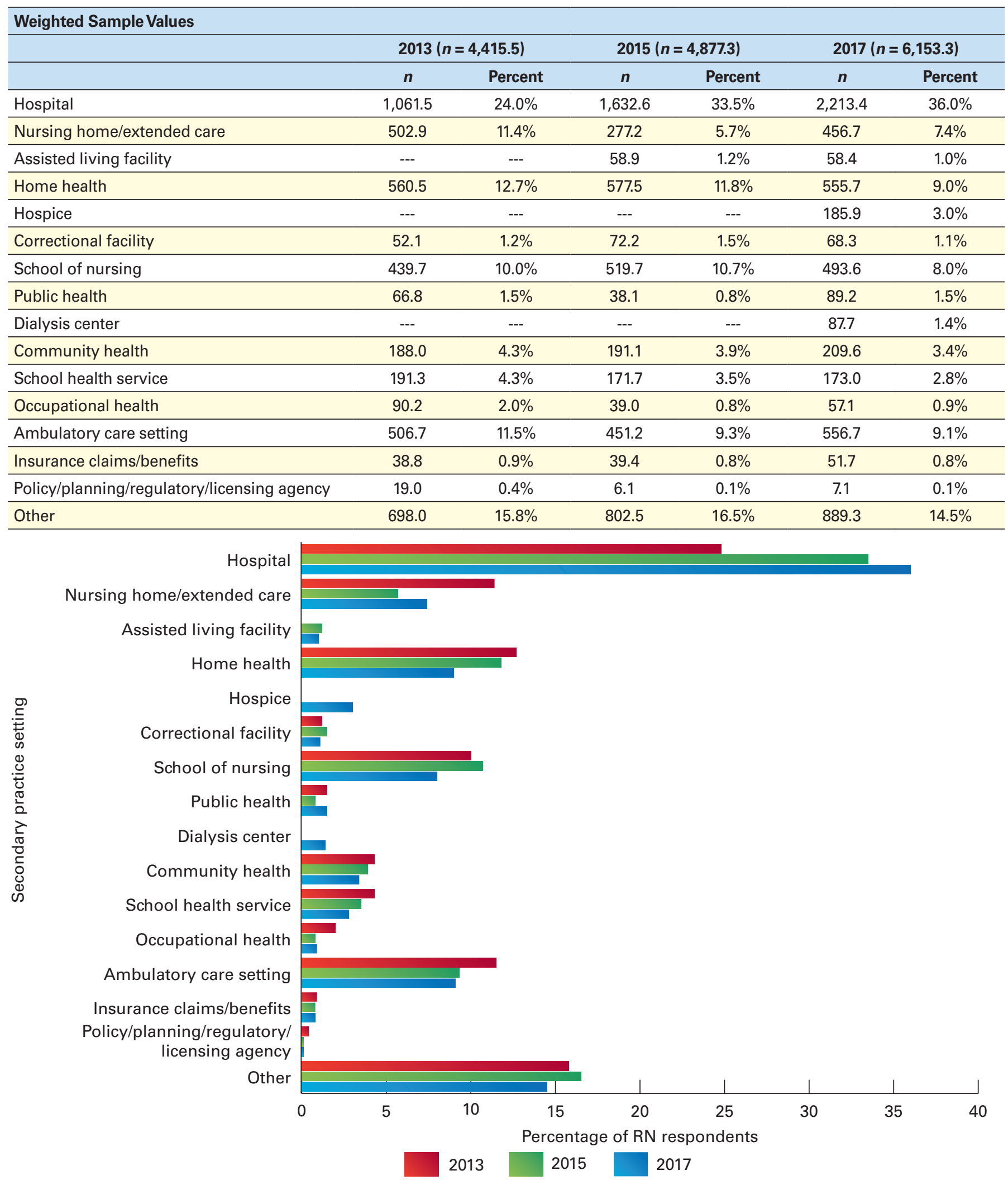

Note. Survey participants were asked to answer this question only if they were actively employed in nursing. 


\section{Secondary nursing position title}

The most frequently selected position title that corresponds to secondary nursing practice position was staff nurse ( $55.8 \%$ ), followed by advanced practice registered nurse (12.1\%), and nurse faculty/educator (9.8\%). The percentage of respondents who selected advanced practice registered nurse increased from 2015 to 2017. About 10\% of respondents selected another category in both 2015 and 2017. See Table 29.

\section{TABLE 29}

\section{Secondary Nursing Position Title-RN}

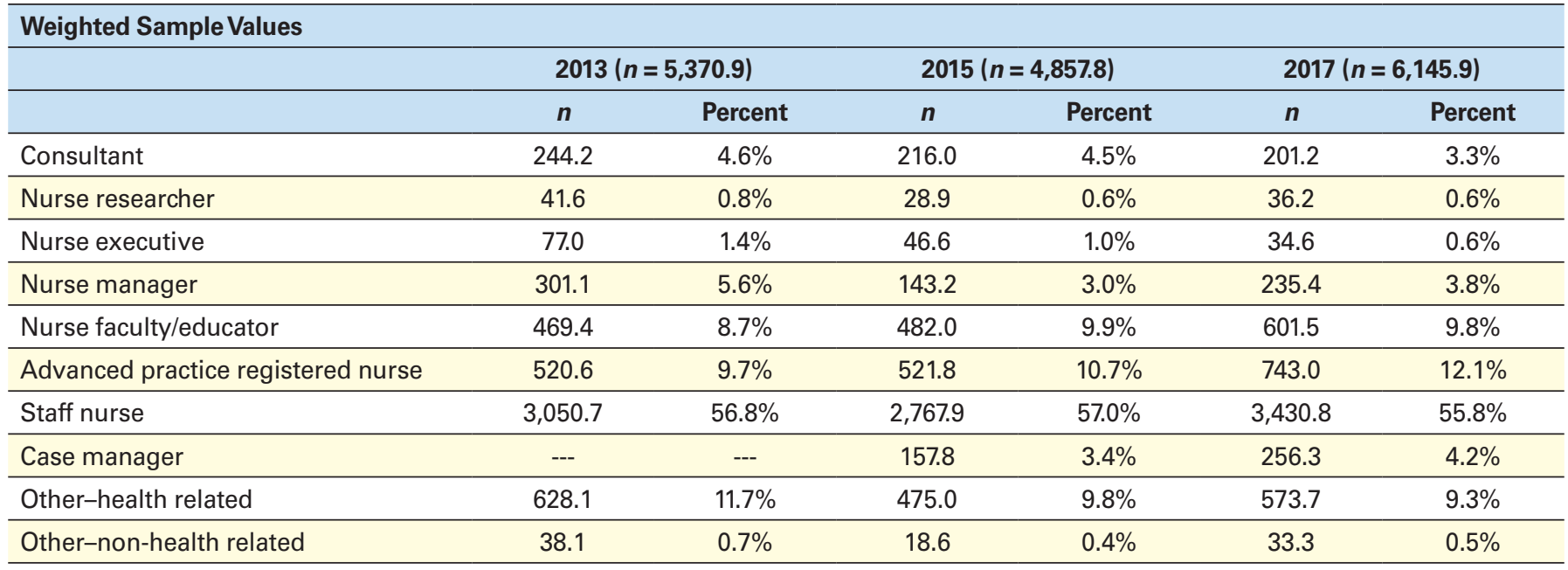

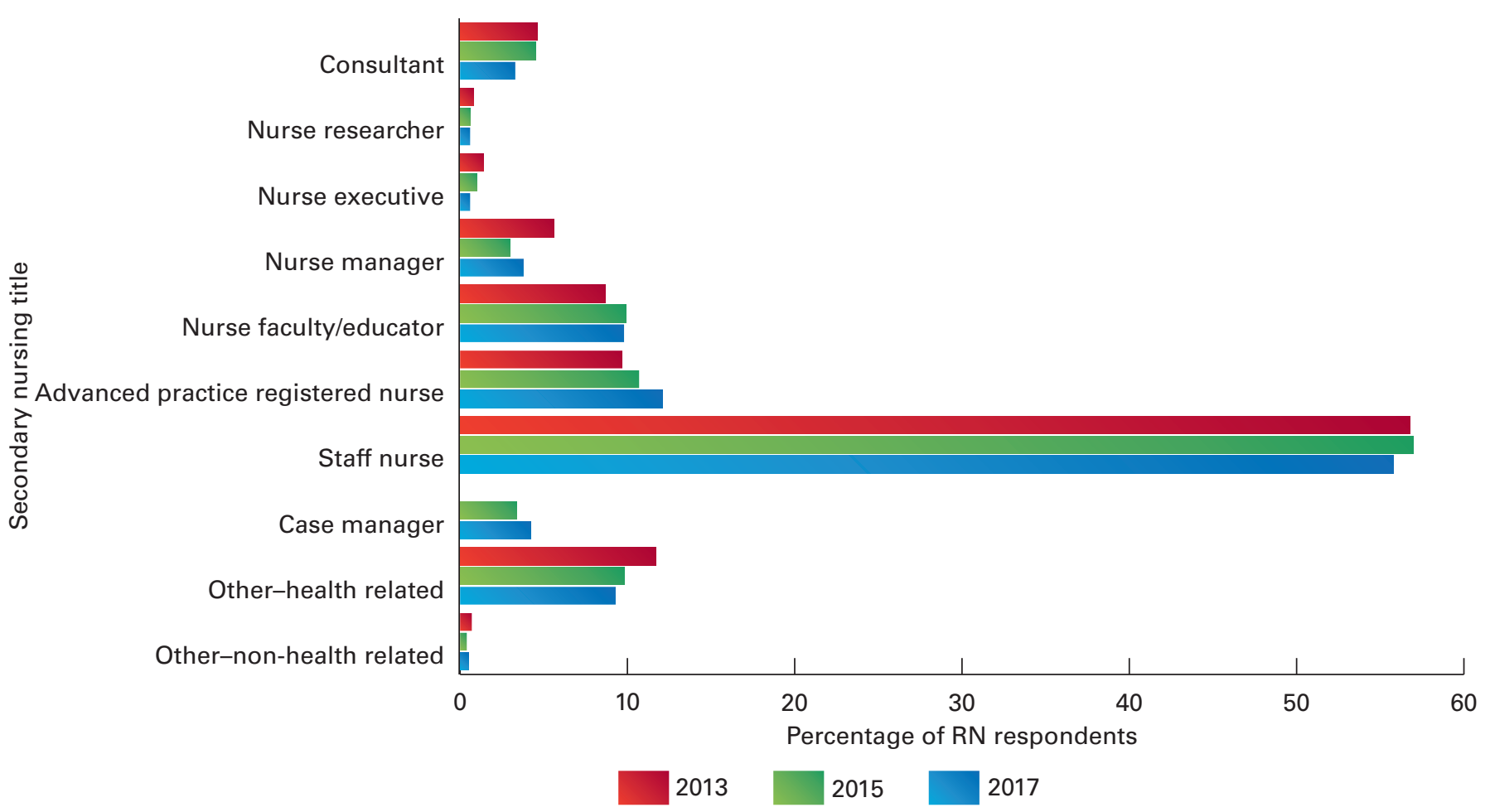

Note. Survey participants were asked to answer this question only if they were actively employed in nursing. 
Approximately $10 \%$ of respondents $(10.2 \%)$ reported acute care/critical care as the specialty for their secondary nursing position. The second most frequently selected specialty was medical-surgical (7.7\%) followed by geriatric/gerontology (7.4\%). See Table 30 .

\section{TABLE 30}

\section{Secondary Nursing Position Specialty-RN}

\section{Weighted Sample Values}

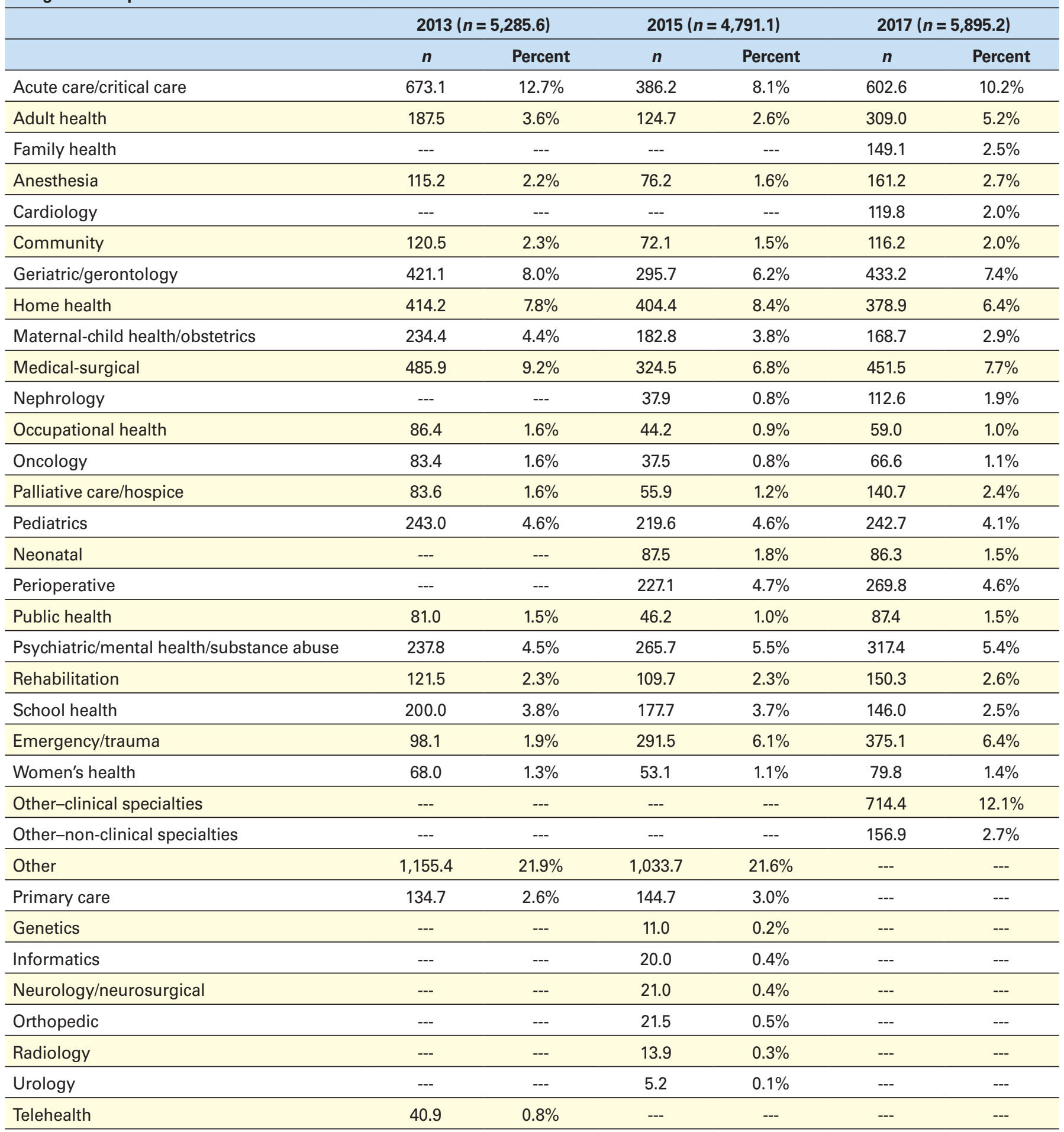




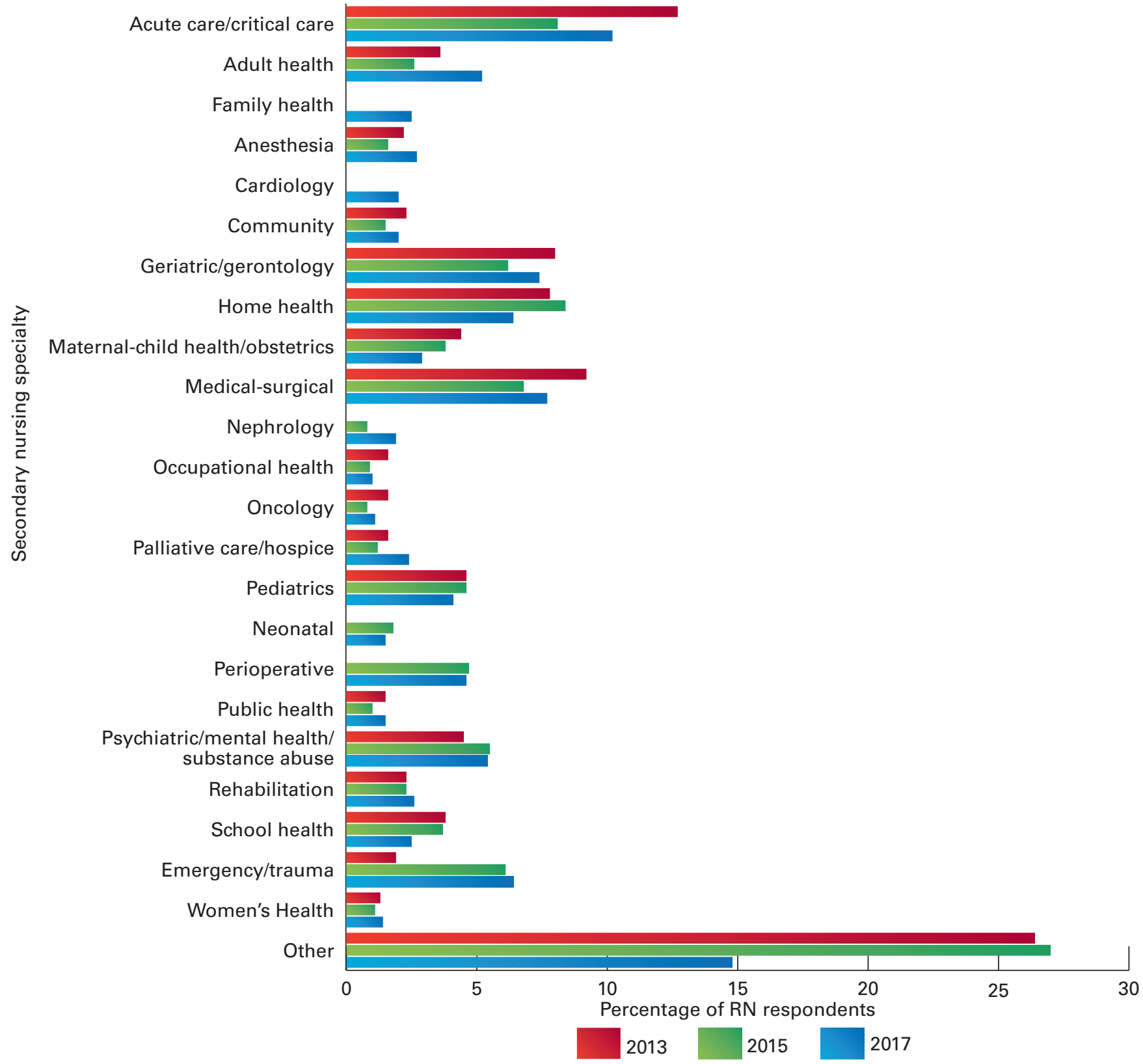

Note. Survey participants were asked to answer this question only if they were actively employed in nursing.

\section{Salary/Earnings}

\section{6 pre-tax annual earnings from primary nursing position}

The median pre-tax annual earnings for responding RNs increased from $\$ 60,000$ in 2015 to $\$ 63,000$ in 2017 . Categorically, the percentage of respondents earning less than $\$ 40,000$ annually decreased by 2.7 percentage points, the percentage making between $\$ 40,000$ and $\$ 60,000$ decreased by 2.9 percentage points, while those making between $\$ 80,000$ and $\$ 100,000$ increased by 1.6 percentage points and those making more than $\$ 100,000$ increased by nearly 4 percentage points. See Table 31 . 


\section{TABLE 31}

\section{Annual Earnings-RN}

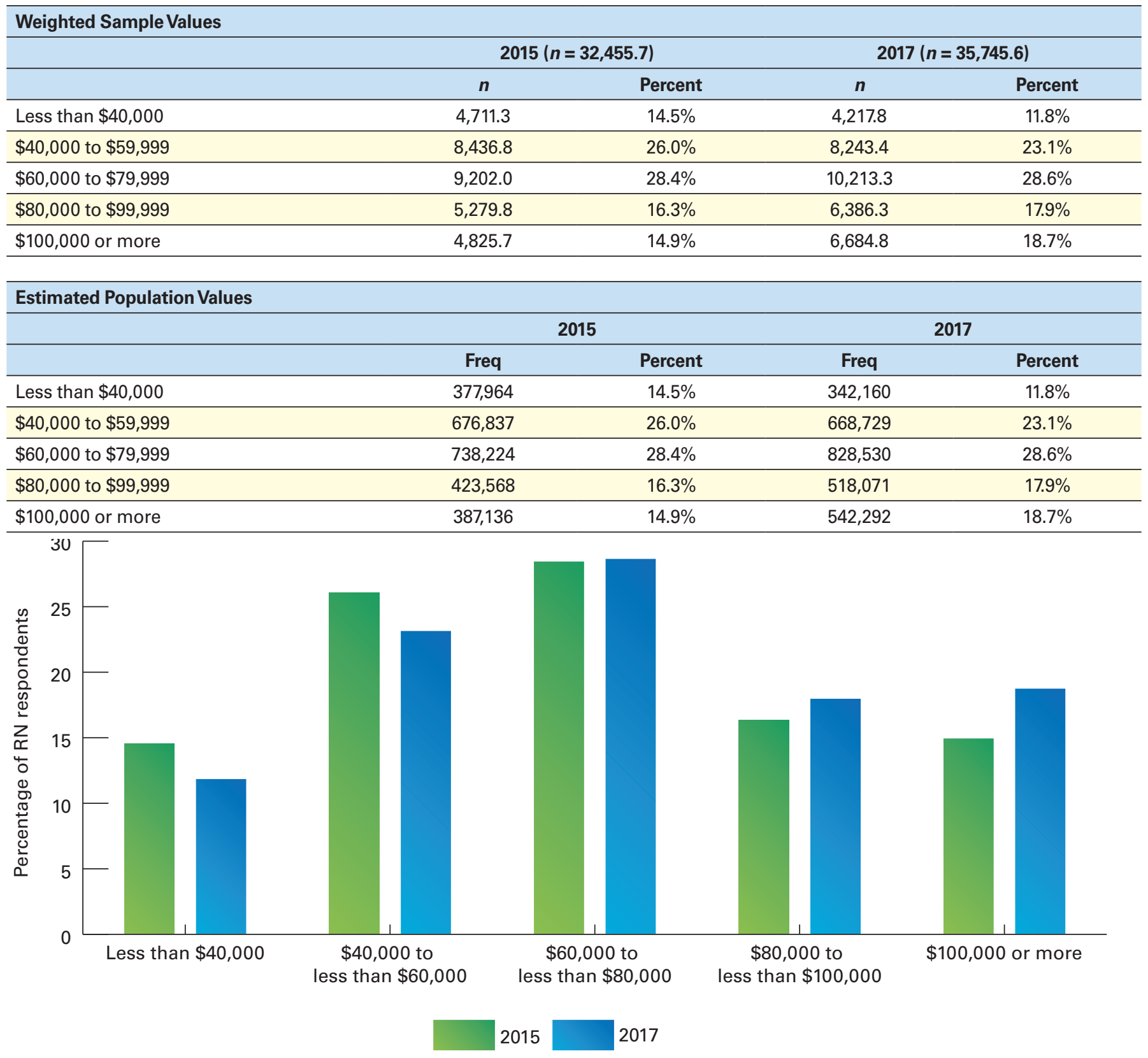

Note. Survey participants were asked to answer this question only if they were actively employed in nursing. Salary includes overtime and bonuses, but does not include sign-on bonuses. APRNs were included in the RN salary tables.

\section{Earnings by gender and specialty}

Looking across all the specialties, the specialty with the highest median salary is anesthesia ( $\$ 170,000$ overall). Across nearly all specialties, men have a higher median salary than women, except for oncology. See Table 32. 
TABLE 32

\section{Median Annual Earnings by Gender and Specialty-RN}

\begin{tabular}{|c|c|c|c|}
\hline & Male & Female & Total \\
\hline \multirow[t]{2}{*}{ Acute care/critical care } & $\$ 76,000$ & $\$ 70,000$ & $\$ 70,000$ \\
\hline & 560 & 3,420 & 3,980 \\
\hline Adult health & 97 & 984 & 1,081 \\
\hline Family health & $\$ 100,000$ & $\$ 82,800$ & $\$ 84,250$ \\
\hline \multirow[t]{2}{*}{ Anesthesia } & $\$ 185,500$ & $\$ 156,200$ & $\$ 170,000$ \\
\hline & 204 & 344 & 548 \\
\hline \multirow[t]{2}{*}{ Cardiology } & $\$ 73,000$ & $\$ 70,000$ & $\$ 70,000$ \\
\hline & 104 & 843 & 947 \\
\hline Community & $\$ 76,200$ & $\$ 70,000$ & $\$ 70,500$ \\
\hline \multirow[t]{2}{*}{ Home health } & $\$ 68,000$ & $\$ 67,000$ & $\$ 68,000$ \\
\hline & 62 & 941 & 1,003 \\
\hline \multirow[t]{2}{*}{ Maternal-child health/obstetrics } & $\$ 76,500$ & $\$ 67,633$ & $\$ 68,000$ \\
\hline & 6 & 1,297 & 1,303 \\
\hline \multirow[t]{2}{*}{ Medical-surgical } & $\$ 70,000$ & $\$ 62,000$ & $\$ 63,000$ \\
\hline & 197 & 2,301 & 2,498 \\
\hline \multirow[t]{2}{*}{ Nephrology } & $\$ 80,000$ & $\$ 74,000$ & $\$ 75,000$ \\
\hline & 49 & 418 & 467 \\
\hline \multirow[t]{2}{*}{ Pediatrics } & $\$ 74,000$ & $\$ 67,000$ & $\$ 67,000$ \\
\hline & 40 & 1,113 & 1,153 \\
\hline \multirow[t]{2}{*}{ Neonatal } & $\$ 80,000$ & $\$ 73,000$ & $\$ 73,000$ \\
\hline & 16 & 595 & 611 \\
\hline \multirow[t]{2}{*}{ Perioperative } & $\$ 84,000$ & $\$ 72,000$ & $\$ 72,550$ \\
\hline & 161 & 1,474 & 1,635 \\
\hline \multirow[t]{2}{*}{ Public health } & $\$ 67,500$ & $\$ 64,490$ & $\$ 65,000$ \\
\hline & 24 & 422 & 446 \\
\hline \multirow[t]{2}{*}{ Psychiatric/mental health/substance abuse } & $\$ 80,000$ & $\$ 70,000$ & $\$ 72,000$ \\
\hline & 185 & 1,054 & 1,239 \\
\hline \multirow[t]{2}{*}{ Rehabilitation } & $\$ 70,000$ & $\$ 65,000$ & $\$ 65,000$ \\
\hline & 55 & 474 & 529 \\
\hline \multirow[t]{2}{*}{ School health } & $\$ 53,000$ & $\$ 50,000$ & $\$ 50,000$ \\
\hline & 14 & 723 & 737 \\
\hline
\end{tabular}




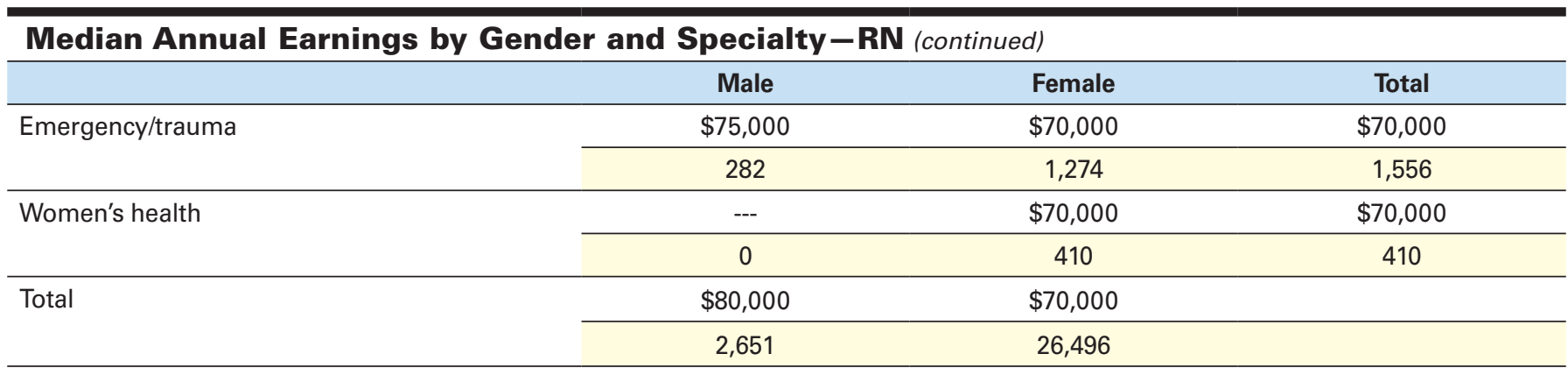

Note. Sample sizes are unweighted. Survey participants were asked to answer this question only if they were actively employed in nursing.

\section{Earnings by highest education}

Upper-level degrees show large increases in median salary, with the highest of $\$ 104,000$ for those with a DNP as their highest degree. See Table 33.

\section{TABLE 33}

\section{Median Annual Earnings by Highest Education-RN}

\begin{tabular}{lcc}
\hline & Median Salary & $\boldsymbol{n}$ \\
\hline Diploma & $\$ 72,900$ & 1,358 \\
\hline Associate degree & $\$ 63,000$ & 8,303 \\
\hline Baccalaureate degree & $\$ 68,000$ & 12,714 \\
\hline Master's degree & $\$ 95,000$ & 4,999 \\
\hline Doctoral degree-PhD & $\$ 100,000$ & 200 \\
\hline Doctoral degree-DNP & $\$ 104,000$ & 413 \\
\hline Doctoral degree-nursing other & $\$ 96,000$ & 37 \\
\hline
\end{tabular}

Note. Sample sizes are unweighted. Survey participants were asked to answer this question only if they were actively employed in nursing.

\section{Earnings by state}

Median earnings rose in most states. The highest median earnings were for RNs practicing in California (\$88,000), Hawaii (\$85,000), New York $(\$ 80,000)$, and Oregon $(\$ 80,000)$. The lowest median earnings were for RNs practicing in South Dakota $(\$ 54,000)$ and Iowa $(\$ 58,000)$. See Table 34 .

\section{TABLE 34}

\section{Median Annual Earnings in Primary Nursing Position by State(s) Where Currently Practicing-RN}

\begin{tabular}{lcc}
\hline & $\mathbf{2 0 1 5}$ & $\mathbf{2 0 1 7}$ \\
\hline Alabama & $\$ 55,000$ & $\$ 60,000$ \\
\hline Alaska & $\$ 70,000$ & $\$ 76,000$ \\
\hline Arizona & $\$ 69,000$ & $\$ 70,500$ \\
\hline Arkansas & $\$ 56,000$ & $\$ 61,605$ \\
\hline California & $\$ 90,000$ & $\$ 88,000$ \\
\hline Colorado & $\$ 63,000$ & $\$ 65,000$ \\
\hline Connecticut & $\$ 75,000$ & $\$ 75,000$ \\
\hline Delaware & $\$ 71,000$ & $\$ 71,900$ \\
\hline District of Columbia & $\$ 75,000$ & $\$ 79,000$ \\
\hline Florida & $\$ 60,000$ & $\$ 65,500$ \\
\hline Georgia & $\$ 64,000$ & $\$ 68,000$ \\
\hline
\end{tabular}

\begin{tabular}{lcc}
\hline & 2015 & $\mathbf{2 0 1 7}$ \\
\hline Nevada & $\$ 72,000$ & $\$ 77,000$ \\
\hline New Hampshire & $\$ 64,000$ & $\$ 66,500$ \\
\hline New Jersey & $\$ 76,000$ & $\$ 75,915$ \\
\hline New Mexico & $\$ 62,000$ & $\$ 69,500$ \\
\hline NewYork & $\$ 77,000$ & $\$ 80,000$ \\
\hline North Carolina & $\$ 58,890$ & $\$ 61,000$ \\
\hline North Dakota & $\$ 54,000$ & $\$ 60,000$ \\
\hline Ohio & $\$ 58,000$ & $\$ 65,000$ \\
\hline Oklahoma & $\$ 58,326$ & $\$ 64,000$ \\
\hline Oregon & $\$ 75,000$ & $\$ 80,000$ \\
\hline Pennsylvania & $\$ 62,000$ & $\$ 70,000$ \\
\hline
\end{tabular}




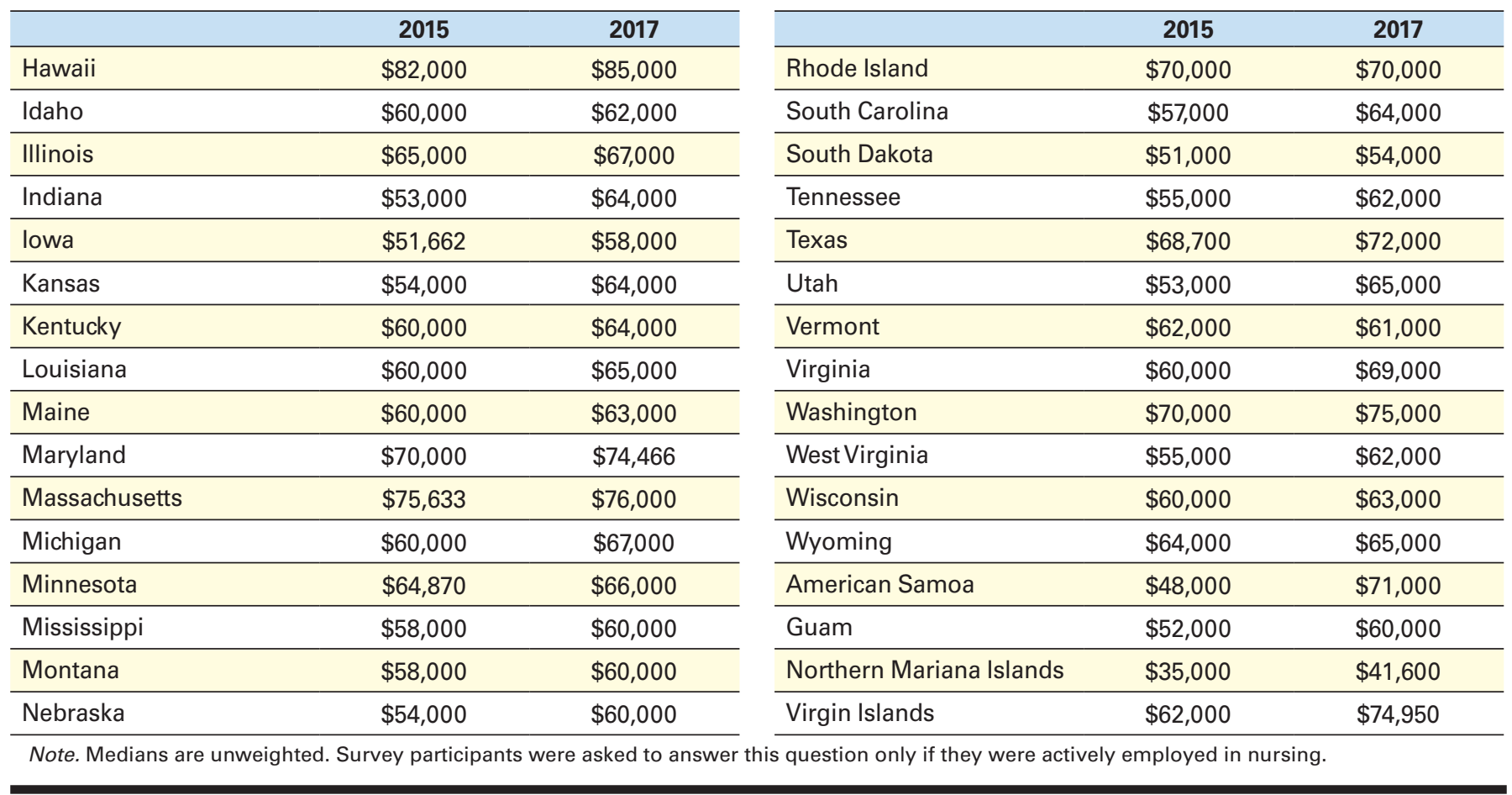

\section{Earnings by years licensed and age}

For all age-groups, there is a steady increase in median salary as number of years licensed increases. Conversely, there is no clear trend upward as nurses age, which indicates that years licensed is more important than age when predicting salary increases. See Table 35.

\section{TABLE 35}

\section{Median Annual Earnings by Years Licensed and Age-RN}

\begin{tabular}{|c|c|c|c|c|c|}
\hline & Licensed 0-1 Years & Licensed 2-5 Years & Licensed 6-10 Years & Licensed 11+ Years & Total \\
\hline \multirow[t]{2}{*}{$<30$} & $\$ 50,000$ & $\$ 55,000$ & $\$ 63,000$ & $\$ 78,000$ & $\$ 55,000$ \\
\hline & 1,151 & 1,888 & 433 & 1 & 3,473 \\
\hline $30-34$ & 311 & 913 & 1,357 & 568 & 3,149 \\
\hline $35-39$ & $\$ 55,000$ & $\$ 60,000$ & $\$ 67,000$ & $\$ 72,000$ & $\$ 67,000$ \\
\hline \multirow[t]{2}{*}{$40-44$} & $\$ 54,000$ & $\$ 60,000$ & $\$ 67,000$ & $\$ 75,000$ & $\$ 70,000$ \\
\hline & 126 & 391 & 458 & 1,979 & 2,954 \\
\hline \multirow[t]{2}{*}{$45-49$} & $\$ 52,000$ & $\$ 60,000$ & $\$ 68,000$ & $\$ 78,000$ & $\$ 74,000$ \\
\hline & 87 & 324 & 359 & 2,522 & 3,292 \\
\hline $50-54$ & $\$ 52,880$ & $\$ 58,000$ & $\$ 66,000$ & $\$ 79,200$ & $\$ 75,000$ \\
\hline \multirow[t]{2}{*}{$60-64$} & $\$ 50,000$ & $\$ 60,000$ & $\$ 63,628$ & $\$ 80,000$ & $\$ 80,000$ \\
\hline & 7 & 49 & 97 & 3,936 & 4,089 \\
\hline \multirow[t]{2}{*}{$\geq 65$} & $\$ 60,000$ & $\$ 68,500$ & $\$ 71,000$ & $\$ 78,000$ & $\$ 78,000$ \\
\hline & 6 & 8 & 16 & 1,672 & 1,702 \\
\hline \multirow[t]{2}{*}{ Total } & $\$ 50,000$ & $\$ 58,000$ & $\$ 66,000$ & $\$ 78,000$ & \\
\hline & 1,961 & 4,435 & 3,888 & 19,433 & \\
\hline
\end{tabular}

Note. Sample sizes are unweighted. Survey participants were asked to answer this question only if they were actively employed in nursing. 


\section{Earnings by APRNs}

Certified registered nurse anesthetists have the highest median salary $(\$ 171,000)$. All APRN certified nurses have a substantially higher median salary than the overall population $(\$ 63,000)$. See Table 36.

\section{TABLE 36}

\section{Median Earnings by APRNs}

\begin{tabular}{|c|c|c|}
\hline & Median Salary & $n$ \\
\hline CNP & $\$ 100,000$ & 2,982 \\
\hline CNS & $\$ 88,000$ & 644 \\
\hline CRNA & $\$ 171,000$ & 557 \\
\hline
\end{tabular}

Note. Sample sizes are unweighted. Survey participants were asked to answer this question only if they were actively employed in nursing. CNP $=$ certified nurse practitioner; $\mathrm{CNS}=$ clinical nurse specialist; $\mathrm{CRNA}=$ certified nurse anesthetist; $\mathrm{CNM}=$ certified nurse midwife.

\section{Telehealth Utilization}

\section{Percent of time providing telehealth}

Survey findings revealed that $54.1 \%$ of responding RNs indicated they were providing nursing services remotely in 2017 compared to $48.8 \%$ in 2015 . The $31.7 \%$ of $\mathrm{RN}$ respondents who were spending up to $25 \%$ of their time providing services remotely was similar to the $31.4 \%$ who indicated such in 2015 . Approximately $10 \%$ of responding RNs indicated that they spend from $76 \%$ to $100 \%$ of their time providing nursing services via phone or electronically to patients in a different location. This represents a 3.8 percentage point increase from 2015. See Table 37.

\section{TABLE 37}

\section{Percent of Time Providing Telehealth - RN}

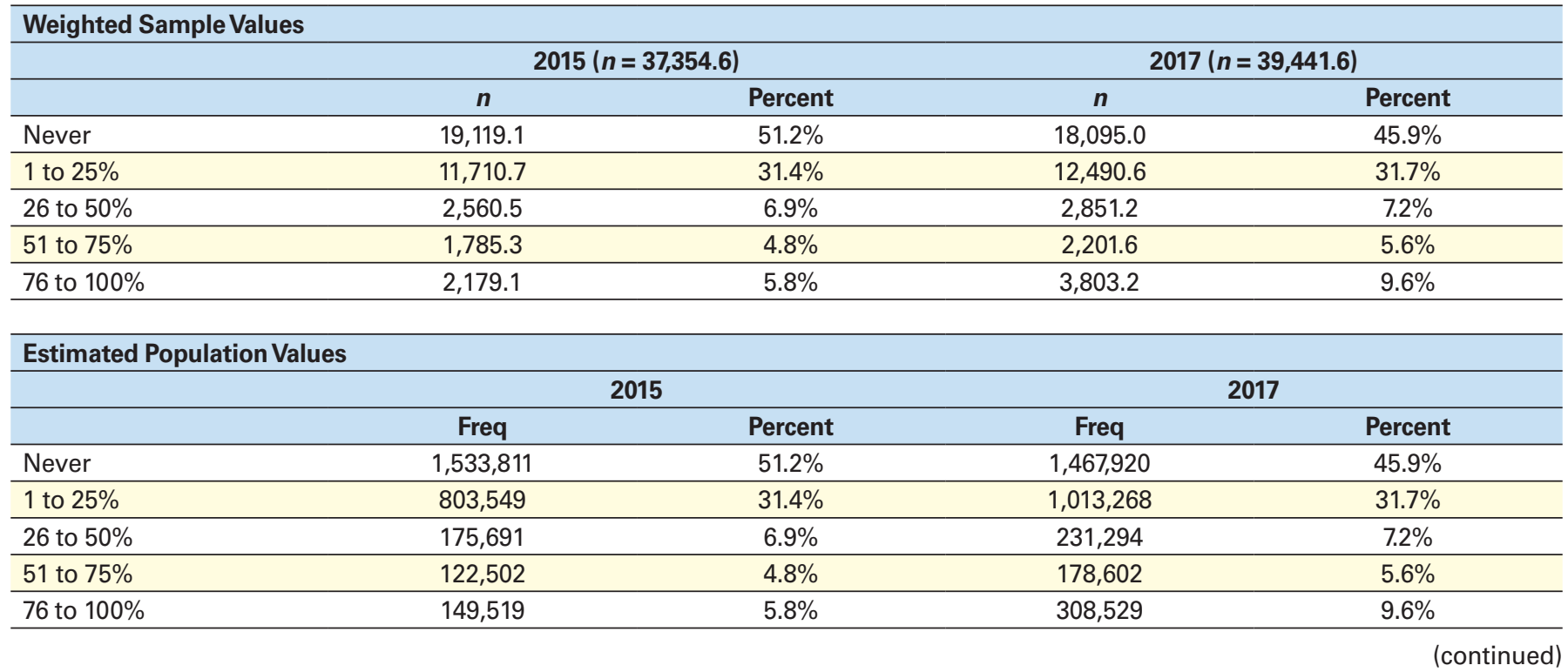




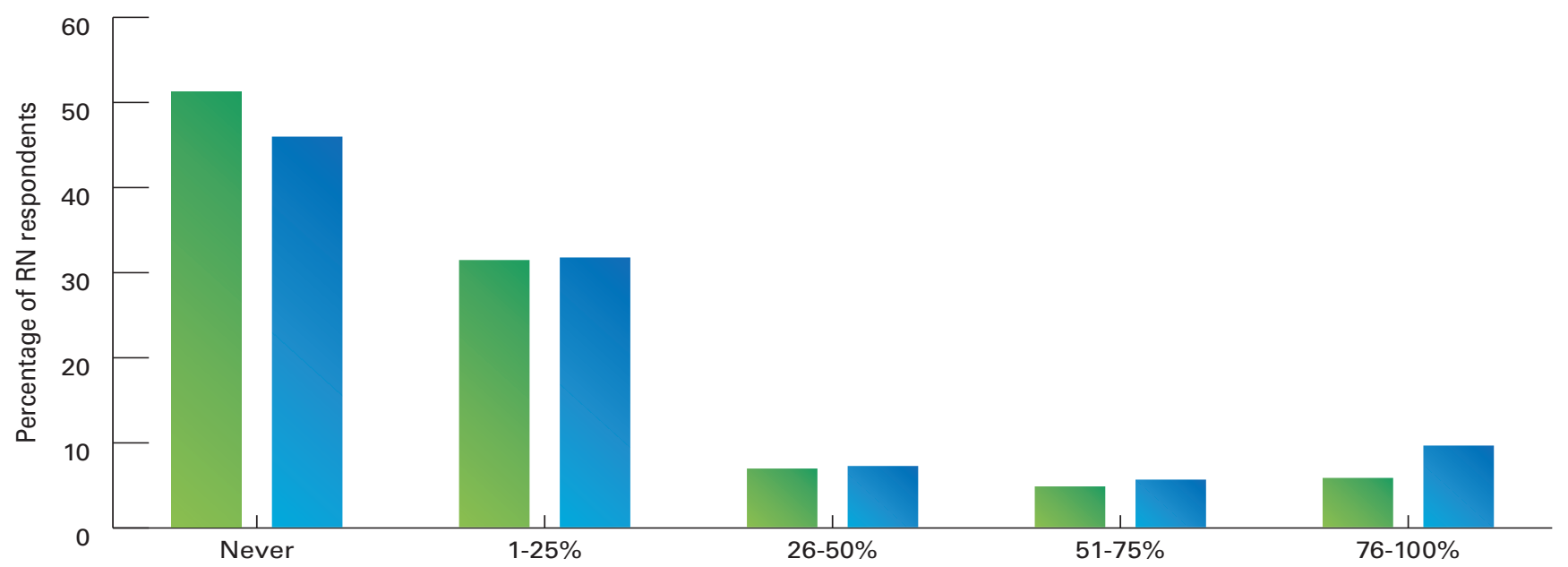

Percent of time spent providing telehealth

2015

Note. Survey participants were asked to answer this question only if they were actively employed in nursing.

\section{Telehealth across state borders}

Of those providing nursing services remotely, $45.7 \%$ of responding RNs were providing phone or electronic services to patients or clients across state borders in 2017, compared to 39.4\% in 2015. Over one-third of RN respondents (35.8\%) provided such services to patients or clients across state borders up to $25 \%$ of the time. This represents a 4.1 percentage point increase from 2015 . See Table 38 .

\section{TABLE 38}

\section{Percent of Time Providing Telehealth Across State Borders-RN}

\begin{tabular}{|c|c|c|c|c|}
\hline \multicolumn{5}{|c|}{ Weighted Sample Values } \\
\hline & \multicolumn{2}{|c|}{$2015(n=18,456.1)$} & \multicolumn{2}{|c|}{$2017(n=17,573.3)$} \\
\hline & $n$ & Percent & $n$ & Percent \\
\hline Never & $11,186.7$ & $60.6 \%$ & $9,535.0$ & $54.3 \%$ \\
\hline 1 to $25 \%$ & $5,843.2$ & $31.7 \%$ & $6,294.5$ & $35.8 \%$ \\
\hline 26 to $50 \%$ & 626.8 & $3.4 \%$ & 692.2 & $3.9 \%$ \\
\hline 51 to $75 \%$ & 298.5 & $1.6 \%$ & 414.0 & $2.4 \%$ \\
\hline
\end{tabular}

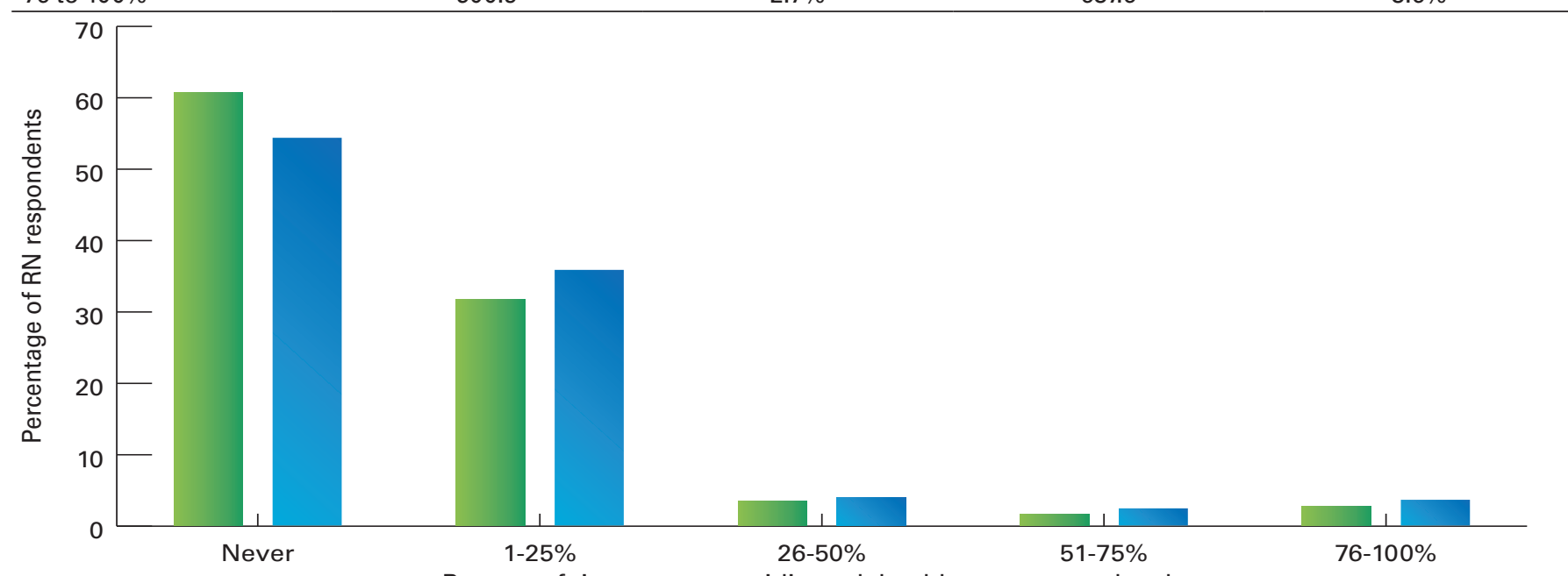

Percent of time spent providing telehealth across state borders

2015

2017

Note. Survey participants were asked to answer this question only if they were actively employed in nursing. 
Only $11.1 \%$ of nurses providing remote services to patients or clients provide such services across national borders. Approximately $9 \%$ of nurses $(9.1 \%)$ reported doing so up to $25 \%$ of their time. See Table 39.

\section{TABLE 39}

\section{Percent of Time Providing Telehealth Across National Borders - RN}

\begin{tabular}{lcccc}
\hline Weighted Sample Values & \multicolumn{2}{c}{} & \multicolumn{2}{c}{$\mathbf{2 0 1 7}(\boldsymbol{n}=\mathbf{1 6 , 3 6 9 . 8 )}$} \\
\hline & $\boldsymbol{n}$ & Percent & $\boldsymbol{n}$ & Percent \\
\hline Never & $16,707.2$ & $92.3 \%$ & $14,548.6$ & $88.9 \%$ \\
\hline 1 to $25 \%$ & $1,194.7$ & $6.6 \%$ & $1,488.2$ & $9.1 \%$ \\
\hline 26 to $50 \%$ & 96.3 & $0.5 \%$ & 129.4 & $0.8 \%$ \\
\hline 51 to $75 \%$ & 33.7 & $0.2 \%$ & 103.7 & $0.6 \%$ \\
\hline 76 to $100 \%$ & 64.3 & $0.4 \%$ & 99.8 & $0.6 \%$ \\
\hline
\end{tabular}

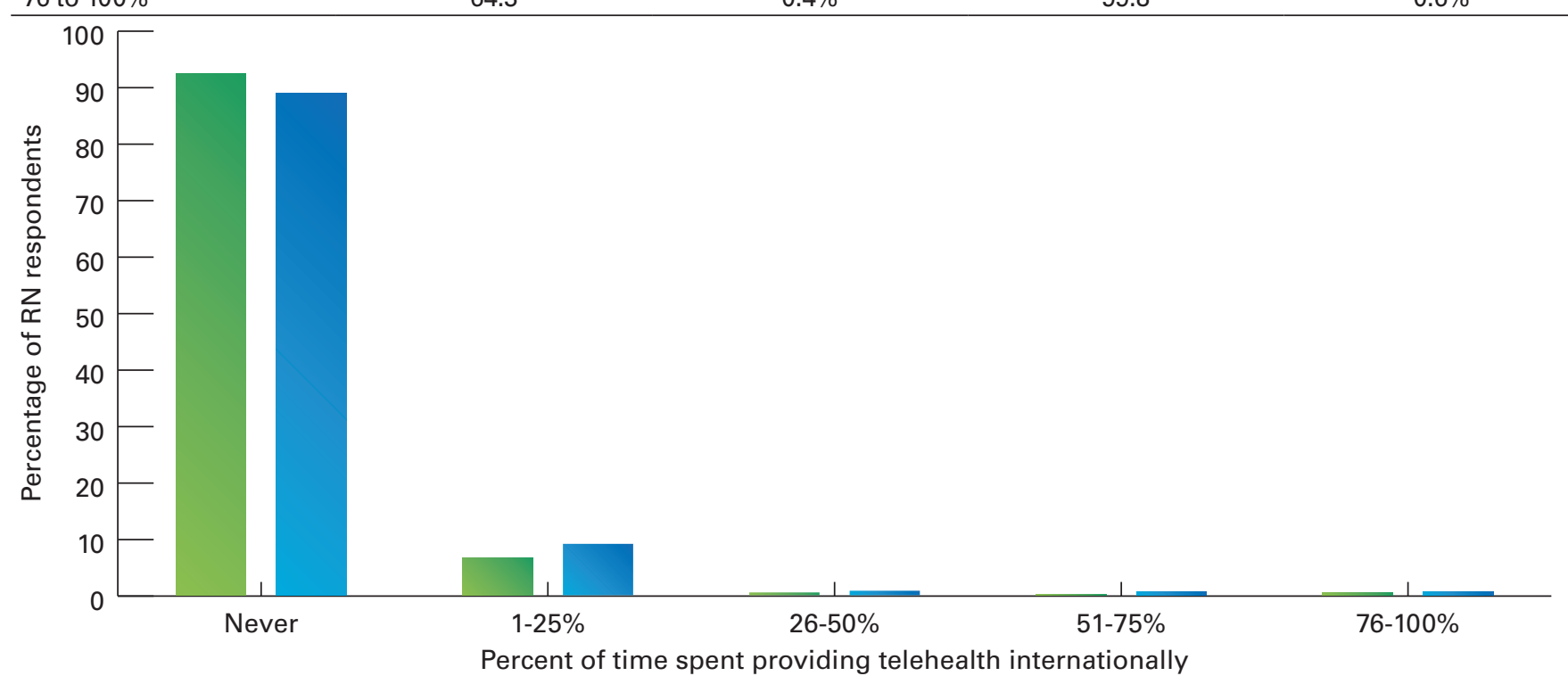

\section{Modes of communication used for telehealth}

Nurses providing services via phone or electronically to patients or clients were also asked to identify the modes of communication used; 94.6\% of these nurses reported using a telephone. This is 2.5 percentage points fewer than in 2015. Nearly one-third (32.7\%) reported using email, which is consistent with the $32.3 \%$ who indicated so in 2015 . Over $20 \%$ reported using electronic messaging in 2017 compared to $18.6 \%$ in 2015 .

With the exception of telephones, a higher proportion of respondents used each of the modes of communication in 2017 than in 2015. See Table 40. 
TABLE 40

\section{Modes of Communication Used for Telehealth - RN}

\begin{tabular}{|c|c|c|c|c|}
\hline \multicolumn{5}{|c|}{ Weighted Sample Values } \\
\hline & \multicolumn{2}{|c|}{$2015(n=15,864.1)$} & \multicolumn{2}{|c|}{$2017(n=17,066.0)$} \\
\hline & $n$ & Percent & $n$ & Percent \\
\hline Electronic messaging & $2,954.0$ & $18.6 \%$ & $3,599.6$ & $21.1 \%$ \\
\hline VolP & 528.7 & $3.3 \%$ & 817.3 & $4.8 \%$ \\
\hline Virtual ICU & 167.7 & $1.1 \%$ & 276.9 & $1.6 \%$ \\
\hline Telephone & $15,406.7$ & $97.1 \%$ & $16,143.9$ & $94.6 \%$ \\
\hline Other & $1,070.9$ & $6.8 \%$ & 926.4 & $5.4 \%$ \\
\hline
\end{tabular}

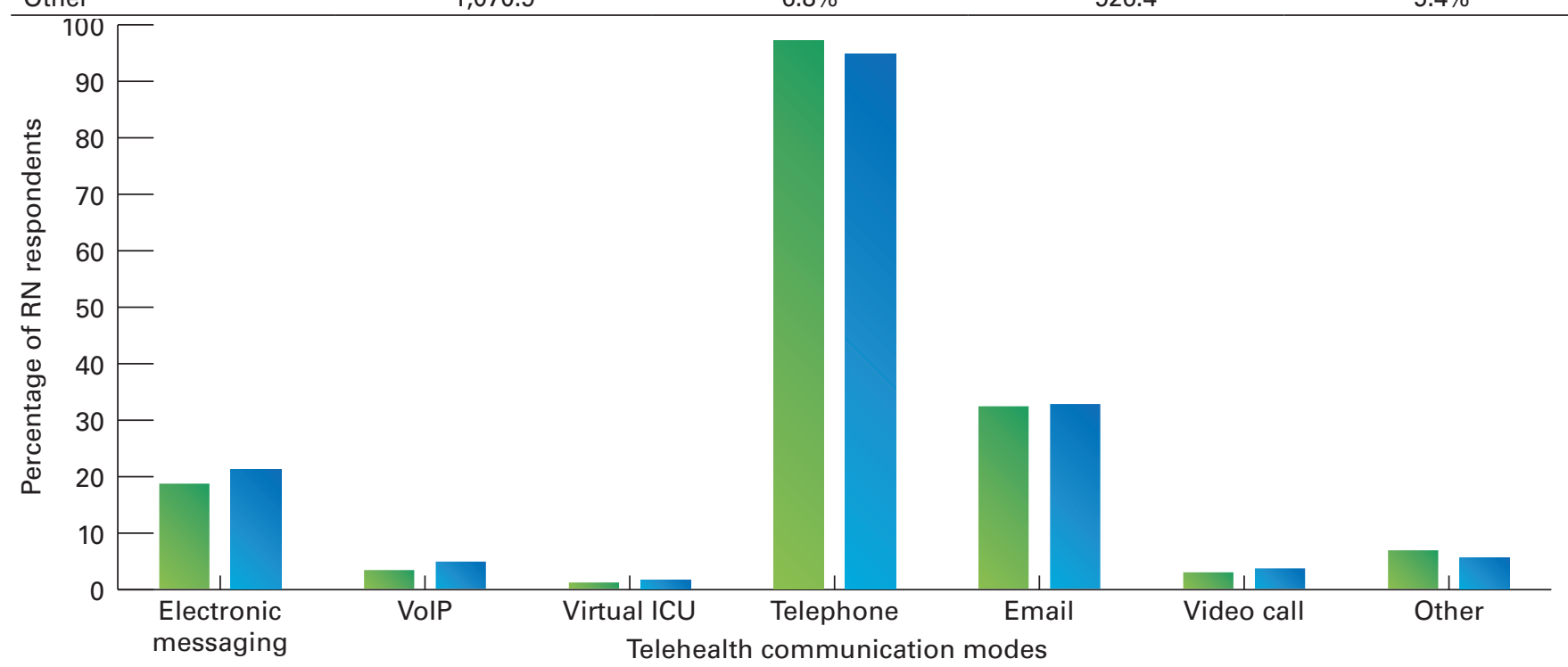

2015

2017

Note. Survey participants were asked to answer this question only if they were actively employed in nursing. Respondents were asked to select all that apply. VoIP = voice over Internet protocol; ICU= intensive care unit.

\section{Discussion and Implications}

This study presents a national, randomized survey of 148,684 licensed RNs. Data collected from the 48,704 respondents (32.8\%) provide the most recent and detailed information on characteristics of the $\mathrm{RN}$ workforce. These data not only illustrate current characteristics and trends of the nursing workforce but also provide valuable information with which to assess progress towards certain goals and priorities outlined by the Institute of Medicine's (IOM; now the National Academy of Medicine) report on the future of nursing (National Academy of Medicine, 2010), including RNs achieving higher levels of education and increased diversity of the nursing workforce.

As of 2017, an estimated 3,951,001 individuals held an active RN license in the United States and its territories (up from 3,728,501 in 2015), representing an increase of 261,275 RN licensees when compared to 2015.

To illustrate trends in workforce supply, the current study's results were compared to national nursing workforce studies that based their findings on data collected years prior to the study publication date. For example, the 2013 National Nursing Workforce study was compared to HRSAs 2010 results, which were collected from RN workforce data collected in 2008. While the current study used a sample of all RN licensees, which included individuals who were not actively employed in nursing, the other studies may not have, such as the study conducted by Buerhaus, Skinner, Auerbach, and Staiger (2017). When comparing the current study's results to those of HRSA 2013, which contained data from the U.S. Census Bureau's American Community Survey, it should be noted the data were from individuals who reported their current occupation as nursing and who currently had or were seeking a job. The HRSA 2017 data cited were for the U.S. working-age population age 16 or older who were currently employed or who were recently employed and were seeking employment (e.g., individuals who were recently laid off). Thus, the comparisons of statistics from various studies presented should be interpreted with the above considerations in mind. 
The average age of RNs is 51, which is largely consistent with previous study findings (2015 and 2013). The median age of RNs in this study was 53 , with $50.9 \%$ of the respondents being $50+$ years, which is notably higher than the $37 \%$ of the full-time equivalent (FTE) RN workforce between age 50 and 69 reported by Buerhaus et al. (2017). In 2015, 12.4\% of the RN respondents were age 65 or older compared to $14.6 \%$ in the current study, an increase of 2.2 percentage points, which indicates a slow but steady growth of RNs who may be heading for retirement. Buerhaus et al (2017) predicted that as the baby boomer cohort of RNs continues to retire in large numbers, years of nursing experience and knowledge they have accumulated will be lost, having a tremendous impact on health care. An aging population, an aging workforce, and reported nursing shortages across the country have heightened discussions among employers about the utilization of internationally educated nurses to fill the gaps they are experiencing within their health care facilities. The current study found that approximately $5 \%$ of the RN workforce obtained their entry-level nursing education outside of the United States. This is consistent with results from 2015 (5.4\%) and $2013(5.0 \%)$.

Evidence on the progress towards the IOM recommendation of increasing the proportion of nurses with a baccalaureate degree to $80 \%$ by the year 2020 has been steadily accumulating over the past 8 years. The trend toward a higher percentage of respondents pursuing a BSN degree as their initial nursing education continued in 2017 , with $41.8 \%$ of the RNs reporting the BSN as the degree that qualified them for their first U.S. nursing license, which reflects a 2.8 percenage point increase when compared to 2015 (39.0\%) and a 6.3 percentage point increase when compared to 2013 (35.5\%). In 2017, 64.1\% of the RNs obtained a baccalaureate or higher degree, which exceeds the $54 \%$ reported in 2016 by the Center to Champion Nursing in America Campaign for Action Dashboard (Campaign Progress, 2017). Enrollment in RN-to-BSN programs is increasing in response to calls from within and outside of the profession for a more highly educated nursing workforce. Many professional practice settings, including Magnet hospitals and academic health centers, now require or prefer the baccalaureate degree for specific nursing roles. From 2015 to 2016, enrollments increased by 1.0 percent, marking the 13th year of increases in RN-to-BSN programs (American Association of Colleges of Nursing [AACN], 2017b).

In terms of graduate education, the number of RNs with a master's degree in nursing has increased from $13.8 \%$ in 2013 to $17.1 \%$ in 2017, an increase of 3.3 percentage points. Although small in terms of percentage, $1.1 \%$ of responding RNs reported having a DNP as their highest level of nursing education, which is double the percent of nurses who reported having a DNP in 2015. According to AACN (2017a), the number of students enrolled in DNP programs went from 21,995 in 2015 to 25,289 in 2016 , a $15.0 \%$ increase. There has been very little movement in terms of the number of RNs with a $\mathrm{PhD}$ in nursing. The number of responding $\mathrm{RNs}$ with a PhD in nursing went from $0.9 \%$ in 2015 to $0.6 \%$ in 2017.

In 2015, the IOM (National Academy of Medicine) released a report in brief, "Assessing Progress on the Institute of Medicine Report on The Future of Nursing" (National Academy of Medicine, 2015), a specific recommendation was to promote diversity in the profession to better represent the patient population it serves, including gender, race, and ethnic diversity. The percentage of male RNs (9.1\%) in the workforce in 2017 increased by 2.5 percentage points when compared with findings from the 2013 study (6.6\%). The 9.1\% male RNs identified in the current study is less than the 12\% male FTE RNs identified by Buerhaus et al. (2017). Approximately $5.3 \%$ of the RNs indicated that they were of Hispanic or Latino origin compared to $17.8 \%$ of the U.S. population (U.S. Census Bureau, 2017a). In the current study $19.2 \%$ of the $\mathrm{RN}$ respondents were minorities, which includes other and two or more races: Asian (7.5\%), Black/African American (6.2\%), other (2.9\%), and two or more races (1.7\%). The percentage of nurses who identify as Asian in the current study exceeds the $5.7 \%$ represented in the U.S. population; the percentage of nurses who identify as Black/African American falls below the $13.3 \%$ in the U.S. population (U.S. Census Bureau, 2017a).

Of all study respondents with a secondary position in nursing, 9.8\% indicated they were nurse faculty compared to $9.9 \%$ in 2015 . The national shortage of nurse faculty contributed to many qualified nursing school applicants in 2016 being turned down (AACN, 2017c). While more full-time and primary nurse faculty are needed, RNs who work part time as nurse faculty in a secondary position are one avenue of increasing nurse faculty numbers.

In the 2017 survey, response options related to primary and secondary practice setting were slightly modified. Hospice and dialysis center were added as new categories. Hospitals were the primary employment setting for $55.7 \%$ of RNs, $54.4 \%$ in 2015 , but less than the $56.5 \%$ reported in 2013 . Ambulatory care setting was the second most frequently selected setting reported by $9.4 \%$ of RNs, followed by nursing home/extended care (4.8\%), and home health (4.3\%). Nurses work setting is changing owing in part to the growing use and acceptance of technology (American Well, 2015; HIMSS Analytics, 2015).

Telehealth is a growing trend in health care delivery and has the potential to affect the nursing workforce as well as nurse licensure issues. This was the second year that items related to telehealth practice by nurses were included in the survey. Respondents were asked to indicate the percentage of time they provided nursing services or communicated with a patient or client located somewhere different from where they were located, via phone or electronically. Results revealed that over half (54.1\%) of responding RNs provided nurse services using telehealth technologies. In the current study, $45.7 \%$ provided these services across a state border compared to $39.4 \%$ in 2015, an increase of 6.3 percentage points, and $11.1 \%$ reported providing remote services to patients or clients across national borders, which is an increase of 3.4 percentage points when compared to $2015(7.7 \%)$. 
These data raise important questions for employers and regulators. NCSBN is working with states to adopt the national Enhanced Nurse Licensure Compact (eNLC), a multistate licensure model that replaces the one-license, one-state model and would allow nurses to communicate and provide services remotely and over long distances (NCSBN, 2018). The eNLC increases access to care while maintaining public protection at the state level. Under the eNLC, eligible nurses would possess a multistate license issued by their home state (state of residence) and would be able to provide care to patients in other compact states without the time and expense of obtaining additional licenses. As of May 2018, 30 states have adopted the eNLC.

The median pre-tax annual earnings for RNs in the current study increased from $\$ 60,000$ in 2015 to $\$ 63,000$ in 2017 , which is lower than the median salary of $\$ 70,000$ for RNs reported by the Bureau of Labor Statistics Occupational Outlook Handbook (U.S. Bureau of Labor Statistics, 2018). In the current study, responding RNs making between $\$ 80,000$ and $\$ 100,000$ increased by 1.6 percentage points and those making more than $\$ 100,000$ increased by nearly 4 percentage points.

\section{Limitations}

Missing data exist for certain variables in certain categories. In an effort to present the data in the most accurate manner, the number of weighted valid responses to each question was reported for every table. Missing data were not imputed; hence, the presented statistics represent the weighted responses from participants who responded to each respective survey item. The data presented on male nurses, foreign-educated nurses, and APRNs represent smaller sample sizes. In general, caution should be taken in interpreting any statistics from a small group or cell size.

\section{Conclusion}

This national randomized survey provides a cross-sectional view of the current RN nursing workforce. The data generated contribute to a better understanding of the supply of nurses today and identify important trends in the national workforce. The workforce of today will change as older nurses retire and sufficient numbers of nurses step in to fill their shoes; the workforce of tomorrow will be slightly younger, highly educated, working in the community providing primary health care, and using technology and telehealth as a means to deliver health care. Advances in technology will continue to raise important questions for employers and regulators. This study also contributes information with which to assess progress towards the goals outlined in the Future of Nursing report (National Academy of Medicine, 2010), including achieving higher levels of education, promoting diversity, and improving data collection concerning the national health workforce. 


\section{Licensed Practical Nurse/Licensed Vocational Nurse Results \\ Gender}

The current study indicated $92.3 \%$ of responding LPNs/LVNs were female and $7.7 \%$ of respondents were male. See Table 41.

TABLE 41

\section{Gender Distribution of Nurses - LPN/LVN}

\begin{tabular}{|c|c|c|c|c|}
\hline \multicolumn{5}{|c|}{ Weighted Sample Values } \\
\hline & \multicolumn{2}{|c|}{$2015(n=28,891.0)$} & \multicolumn{2}{|c|}{$2017(n=34,616.8)$} \\
\hline & $n$ & Percent & $n$ & Percent \\
\hline Female & $26,721.3$ & $92.5 \%$ & $31,945.9$ & $92.3 \%$ \\
\hline & Freq & Percent & Freq & Percent \\
\hline Male & 65,246 & $7.5 \%$ & 61,064 & $7.7 \%$ \\
\hline Female & 803,559 & $92.5 \%$ & 730,383 & $92.3 \%$ \\
\hline
\end{tabular}

\section{Race/Ethnicity}

One of the changes to the 2017 survey included the modification of the race/ethnicity question. Previously, race and ethnicity categories were listed in one question. The question was modified so that ethnicity and race are now two separate questions. Respondents were first asked whether they were of Hispanic or Latino origin and then asked to identify their race. Anyone who selected more than one race category was recoded into the two or more races category..

Approximately $71 \%$ of responding LPNs/LVNs were White/Caucasian compared to $81 \%$ of responding RNs. Almost three times as many responding LPNs/LVNs were Black/African American (18.5\%) compared to RNs (6.2\%). See Tables 42 and 43.

The LPN/LVN workforce is also more ethnically diverse than the $\mathrm{RN}$ workforce, with $7.4 \%$ of responding LPNs/LVNs indicating that they were of Hispanic or Latino origin compared to $5.3 \%$ of RNs.

\section{TABLE 42}

\section{Race/Ethnicity-LPN/LVN}

\begin{tabular}{|c|c|c|c|c|}
\hline \multicolumn{5}{|l|}{ Weighted Sample Values } \\
\hline & \multicolumn{2}{|c|}{$2015(n=28,656.3)$} & \multicolumn{2}{|c|}{$2017(n=34,467.5)$} \\
\hline & $n$ & Percent & $n$ & Percent \\
\hline Asian & $1,583.2$ & $5.5 \%$ & 897.4 & $2.6 \%$ \\
\hline Black/ African American & $4,689.5$ & $16.4 \%$ & $6,372.4$ & $18.5 \%$ \\
\hline White/Caucasian & $20,839.7$ & $72.7 \%$ & $24,604.0$ & $71.4 \%$ \\
\hline Other race & 429.2 & $1.5 \%$ & $1,568.5$ & $4.6 \%$ \\
\hline Two or more races & 702.6 & $2.5 \%$ & 743.1 & $2.2 \%$ \\
\hline \multicolumn{5}{|l|}{ Estimated Population Values } \\
\hline Asian & 47,610 & $5.5 \%$ & 20,517 & $2.6 \%$ \\
\hline Black/ African American & 141,021 & $16.4 \%$ & 145,692 & $18.5 \%$ \\
\hline Native Hawaiian or other Pacific Islander & 4,343 & $0.5 \%$ & 1,427 & $0.2 \%$ \\
\hline
\end{tabular}

S46 Journal of Nursing Regulation 


\section{Race/Ethnicity-LPN/LVN (continued)}

\begin{tabular}{|c|c|c|c|c|}
\hline \multicolumn{5}{|c|}{ Estimated Population Values } \\
\hline & \multicolumn{2}{|c|}{2015} & \multicolumn{2}{|c|}{2017} \\
\hline & Freq & Percent & Freq & Percent \\
\hline Other race & 12,906 & $1.5 \%$ & 35,860 & $4.6 \%$ \\
\hline Two or more races & 21,129 & $2.5 \%$ & 16,990 & $2.2 \%$ \\
\hline
\end{tabular}

Note. In the 2013 and 2015 surveys, the Hispanic/Latino origin and race categories were combined into one question. For the race question, respondents were asked to select all that apply. The responses were subsequently recoded to ensure that the race categories were mutually exclusive. Respondents selecting multiple race categories were reclassified into the 'Two or more races' category.

\section{TABLE 43}

\section{Ethnicity of Hispanic or Latino Origin-LPN/LVN}

\begin{tabular}{|c|c|c|c|c|}
\hline \multicolumn{5}{|l|}{ Weighted Sample Values } \\
\hline & \multicolumn{2}{|c|}{$2015(n=30,620.8)$} & \multicolumn{2}{|c|}{$2017(n=34,449.3)$} \\
\hline & $n$ & Percent & $n$ & Percent \\
\hline Not of Hispanic or Latino origin & $28,656.3$ & $93.6 \%$ & $31,890.6$ & $92.6 \%$ \\
\hline & Freq & Percent & Freq & Percent \\
\hline Hispanic or Latino origin & 59,079 & $6.4 \%$ & 58,498 & $7.4 \%$ \\
\hline Not of Hispanic or Latino origin & 861,746 & $93.6 \%$ & 729,119 & $92.6 \%$ \\
\hline
\end{tabular}

Hispanic or Latino origin

American Indian or Alaska Native

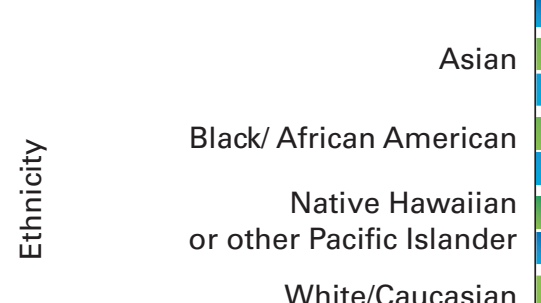

White/Caucasian

Other

Two or more races
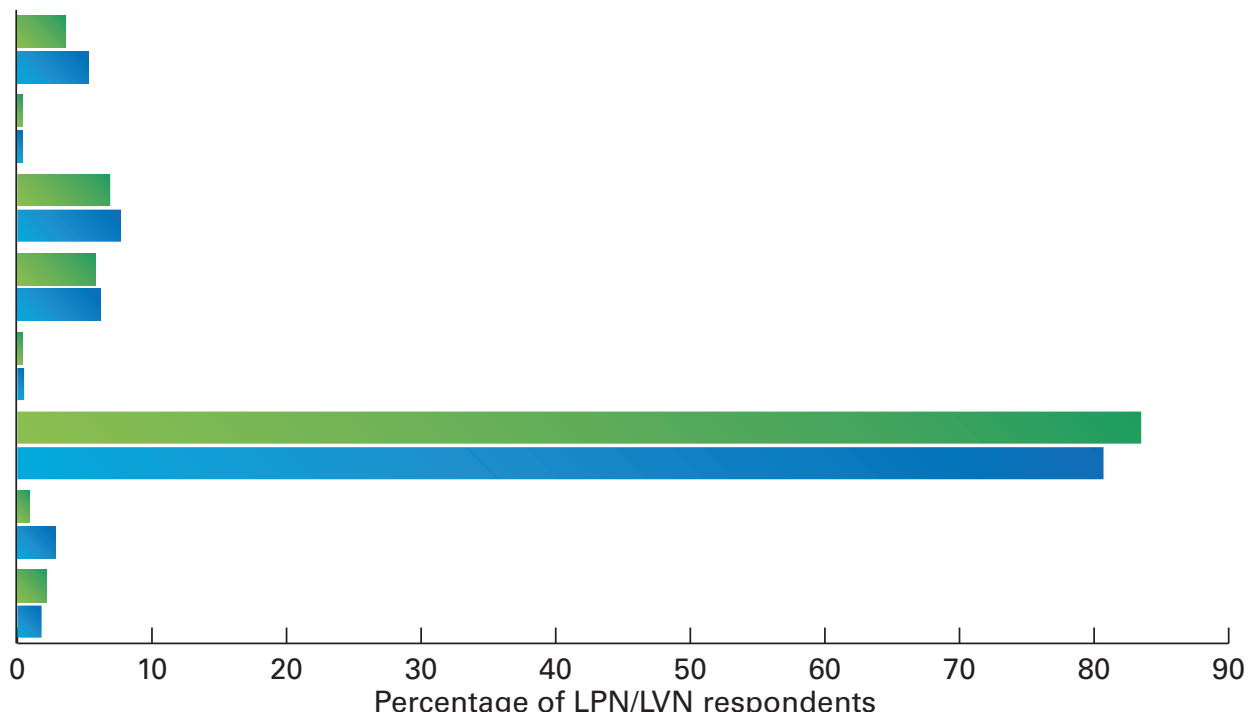

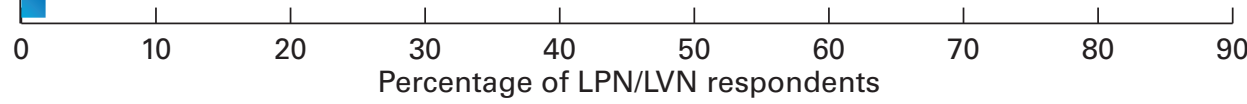

2015

2017

Note. In the 2013 and 2015 surveys, the Hispanic/Latino origin and race categories were combined into one question. For the race question, respondents were asked to select all that apply. The responses were subsequently recoded to ensure that the race categories were mutually exclusive. Respondents selecting multiple race categories were reclassified into the 'Two or more races' category. 
Race/ethnicity by gender

Results show racial groups other than White/Caucasian are gender diverse. Among White/Caucasian nurses, the percentage of men is $5.8 \%$. This is in stark contrast to all other race and ethnic groups, ranging from $9.7 \%$ (American Indian or Alaska Native) to $19.2 \%$ (Asian). See Table 44.

\section{TABLE 44}

\section{Race/Ethnicity by Gender-LPN/LVN}

\begin{tabular}{|c|c|c|c|}
\hline \multicolumn{4}{|l|}{ Weighted Sample Values } \\
\hline & Male & Female & $n$ \\
\hline American Indian or Alaska Native & 18.9 & 176.7 & 195.6 \\
\hline \multirow[t]{2}{*}{ Asian } & 169.6 & 714.8 & 884.3 \\
\hline & $19.2 \%$ & $80.8 \%$ & \\
\hline Black/African American & 606.5 & $5,596.4$ & $6,202.9$ \\
\hline \multirow[t]{2}{*}{ Native Hawaiian or other Pacific Islander } & 7.6 & 46.8 & 54.4 \\
\hline & $14.0 \%$ & $86.0 \%$ & \\
\hline \multirow[t]{2}{*}{ White/Caucasian } & $1,353.9$ & $21,810.5$ & $23,164.4$ \\
\hline & $5.8 \%$ & $94.2 \%$ & \\
\hline Hispanic/Latino of any race & 373.2 & $2,173.2$ & $2,546.4$ \\
\hline \multirow[t]{2}{*}{ Two or more races } & 45.9 & 597.0 & 642.9 \\
\hline & $7.1 \%$ & $92.9 \%$ & \\
\hline \multirow[t]{2}{*}{ Total } & $2,641.0$ & $31,708.6$ & $34,349.6$ \\
\hline & $7.7 \%$ & $92.3 \%$ & \\
\hline
\end{tabular}

Note. In the 2013 and 2015 surveys, the Hispanic/Latino origin and race categories were combined into one question. For the race question, respondents were asked to select all that apply. The responses were subsequently recoded to ensure that the race categories were mutually exclusive. Respondents selecting multiple race categories were reclassified into the 'Two or more races' category.

\section{Age}

In 2017, the median age of LPN/LVN respondents was 54. This is 1 year older than the median age in 2015. More than $50 \%$ of respondents were age 50 or older, which is a four percentage point increase from 2015 (46.1\%); $13.2 \%$ of respondents were age 65 or older in 2017 compared to $9.9 \%$ in 2015 . See Table 45.

\section{TABLE 45}

\section{Age Distribution-LPN/LVN}

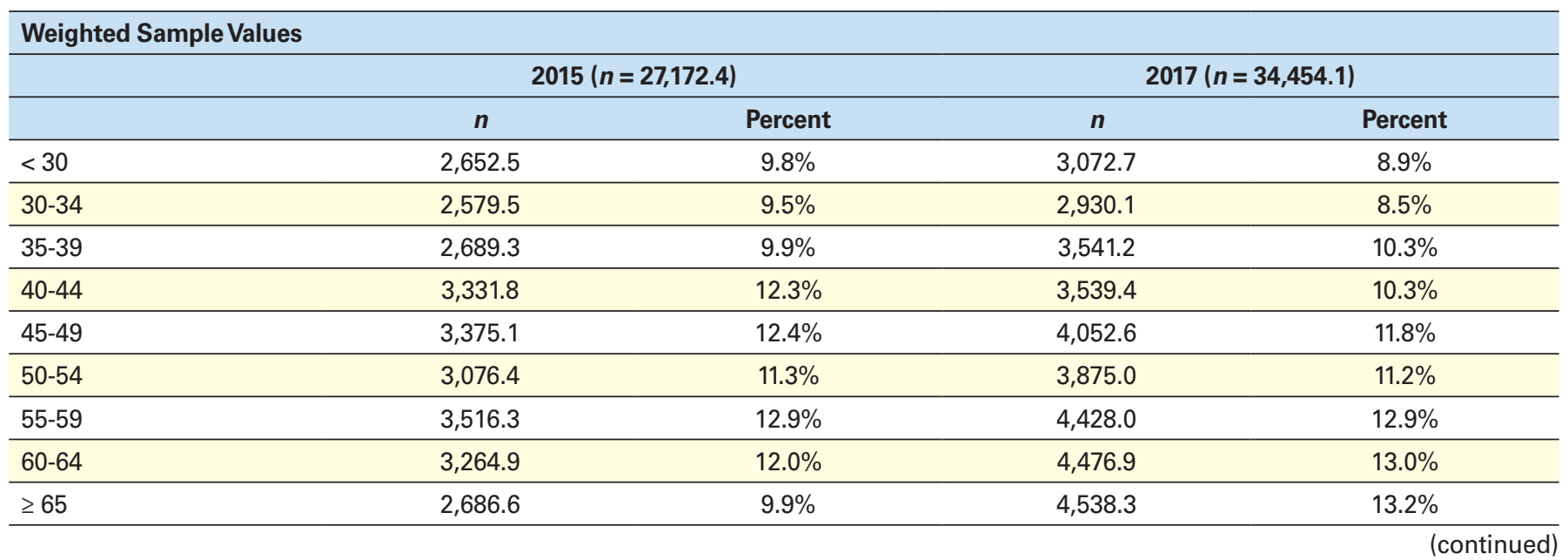

S48 Journal of Nursing Regulation 


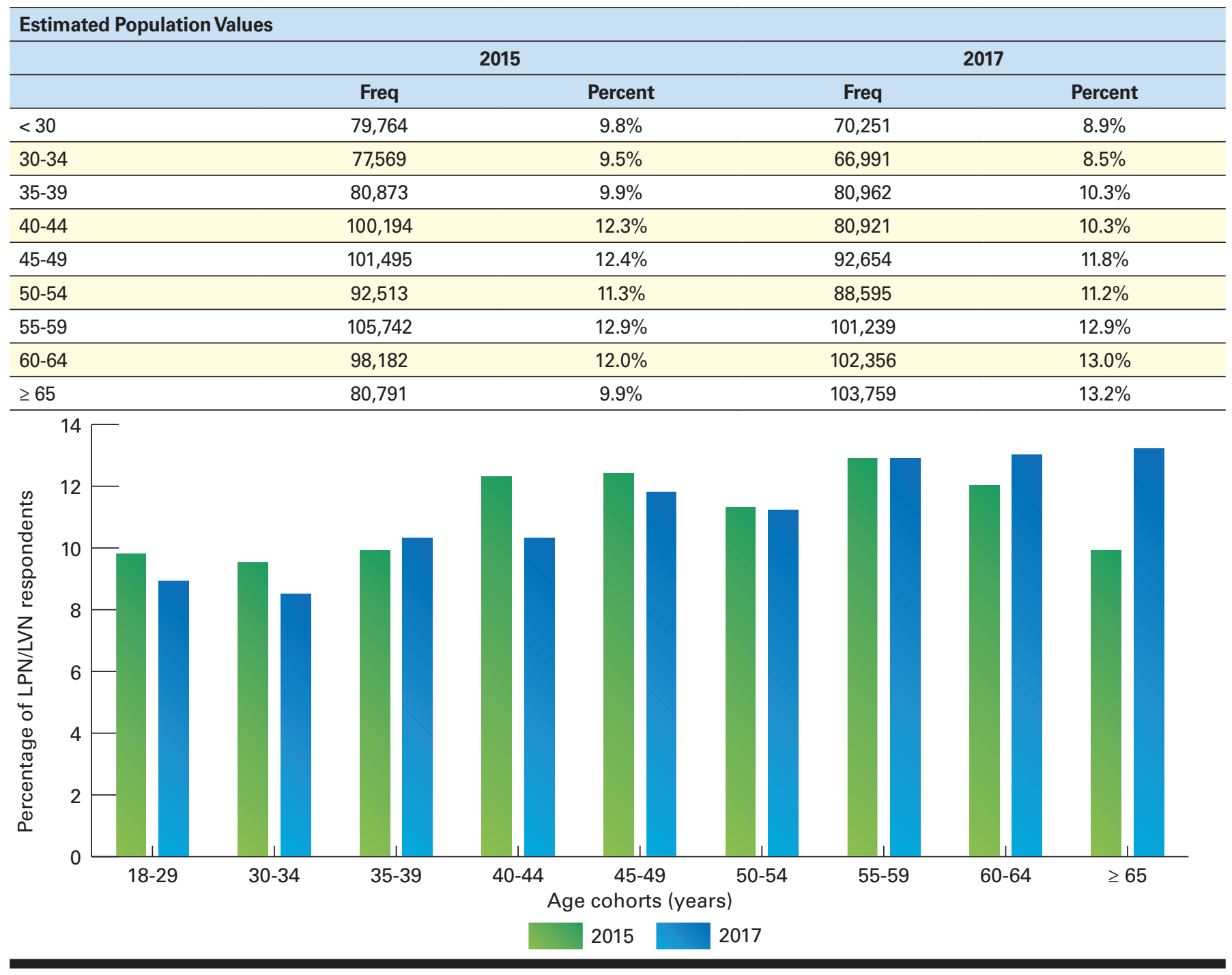

Age by gender

Among older age-groups (ages 55+), women comprise a much higher proportion than in younger age-groups (ages 30 to 54 ). See Table 46.

\section{TABLE 46}

\section{Age Distribution by Gender-LPN/LVN}

\begin{tabular}{|c|c|c|c|c|c|c|c|c|c|c|}
\hline \multicolumn{11}{|c|}{ Weighted Sample Values } \\
\hline Age cohort & $<30$ & $30-34$ & $35-39$ & $40-44$ & $45-49$ & $50-54$ & $55-59$ & $60-64$ & $\geq 65$ & $n$ \\
\hline Male & 303.2 & 218.8 & 263.6 & 286.3 & 349.9 & 360.7 & 291.3 & 305.3 & 220.9 & $2,600.0$ \\
\hline \multirow[t]{2}{*}{ Female } & $2,732.4$ & $2,676.0$ & $3,231.3$ & $3,214.9$ & $3,637.8$ & $3,476.8$ & $4,061.3$ & $4,104.1$ & $4,196.6$ & $31,331.2$ \\
\hline & $8.7 \%$ & $8.5 \%$ & $10.3 \%$ & $10.3 \%$ & $11.6 \%$ & $11.1 \%$ & $13.0 \%$ & $13.1 \%$ & $13.4 \%$ & \\
\hline Total & $8.9 \%$ & $8.5 \%$ & $10.3 \%$ & $10.3 \%$ & $11.8 \%$ & $11.3 \%$ & $12.8 \%$ & $13.0 \%$ & $13.0 \%$ & \\
\hline
\end{tabular}

\section{Age by race/ethnicity}

Hispanic LPNs/LVNs are more likely to be in age-groups under 40 as compared to other race and ethnic groups. See Table 47. 


\section{TABLE 47}

\section{Age Distribution by Race/Ethnicity-LPN/LVN}

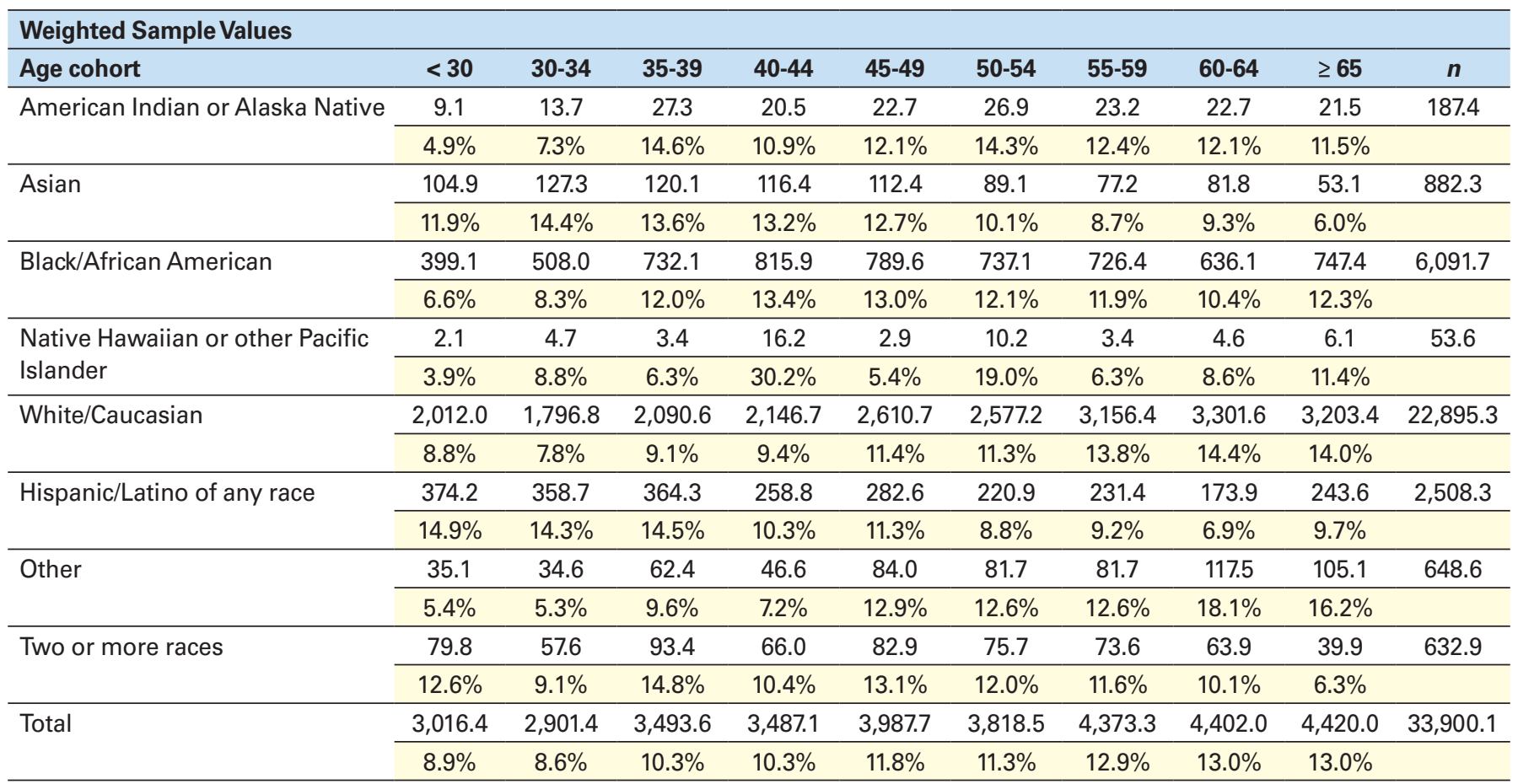

Note. In the 2013 and 2015 surveys, the Hispanic/Latino origin and race categories were combined into one question. For the race question, respondents were asked to select all that apply. The responses were subsequently recoded to ensure that the race categories were mutually exclusive. Respondents selecting multiple race categories were reclassified into the 'Two or more races' category.

\section{Education}

\section{Type of nursing degree or credential for first U.S. nursing license}

In this survey, $83.2 \%$ of respondents indicated that a vocational/practical certificate in nursing qualified them for their first U.S. nursing license. An additional $12.0 \%$ reported that a diploma in nursing qualified them for their first U.S. nursing license, while $4.5 \%$ indicated that it was an associate degree in nursing. See Table 48.

\section{TABLE 48}

\section{Type of Nursing Degree or Credential for First U.S. Nursing License-LPN/LVN}

\begin{tabular}{|c|c|c|c|c|}
\hline \multicolumn{5}{|l|}{ Weighted Sample Values } \\
\hline & \multicolumn{2}{|c|}{$2015(n=30,231.1)$} & \multicolumn{2}{|c|}{$2017(n=34,108.8)$} \\
\hline & $n$ & Percent & $n$ & Percent \\
\hline Diploma & $3,661.3$ & $12.1 \%$ & $4,098.6$ & $12.0 \%$ \\
\hline Associate degree & $1,168.8$ & $3.9 \%$ & $1,521.3$ & $4.5 \%$ \\
\hline Baccalaureate degree & 135.8 & $0.5 \%$ & 93.9 & $0.3 \%$ \\
\hline \multicolumn{5}{|l|}{ Estimated Population Values } \\
\hline & \multicolumn{2}{|c|}{2015} & \multicolumn{2}{|c|}{2017} \\
\hline & Freq & Percent & Freq & Percent \\
\hline Vocational/practical certificate & 759,537 & $83.6 \%$ & 649,197 & $83.2 \%$ \\
\hline Diploma & 110,101 & $12.1 \%$ & 93,707 & $12.0 \%$ \\
\hline
\end{tabular}




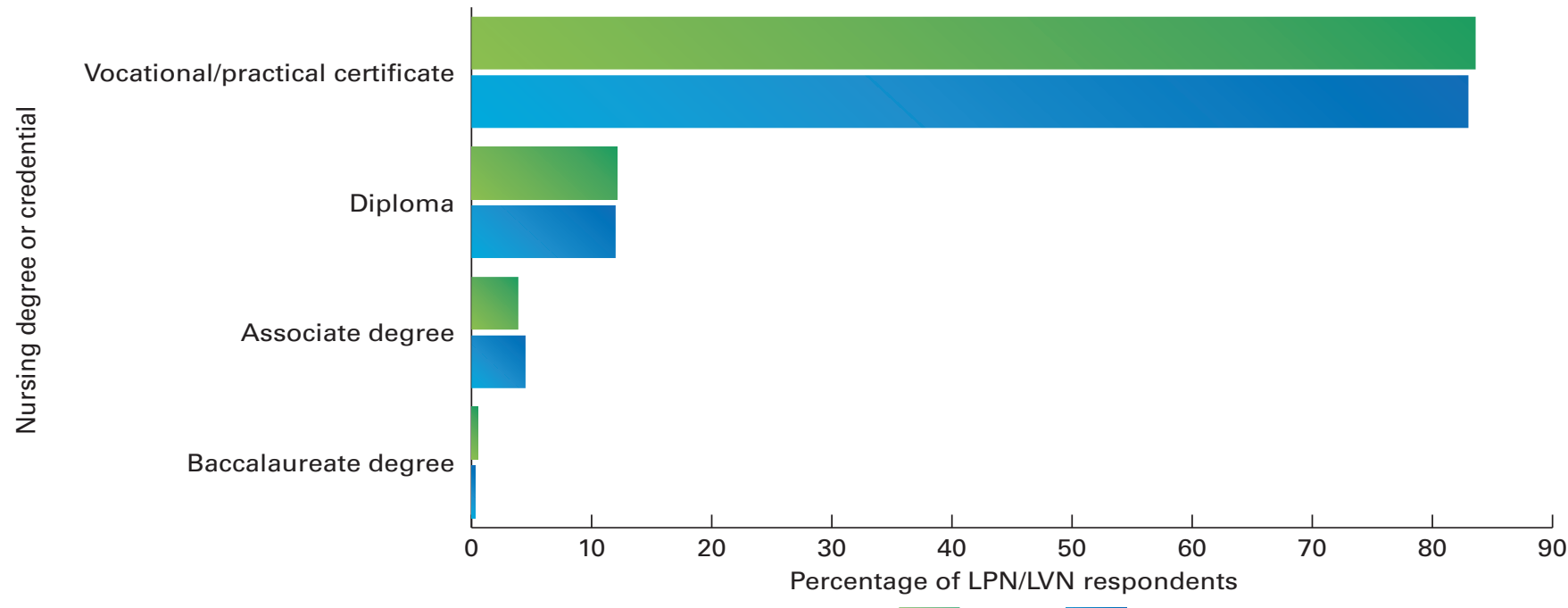

Type of nursing degree or credential for first U.S nursing license by age. Those whose initial education to receive their license was an associate degree were more likely to be in the younger age-groups. This is opposite of the trend with RNs, where those whose initial education was an associate degree were more likely to be older. See Table 49.

TABLE 49

Type of Nursing Degree or Credential for First U.S. Nursing License by Age-LPN/LVN

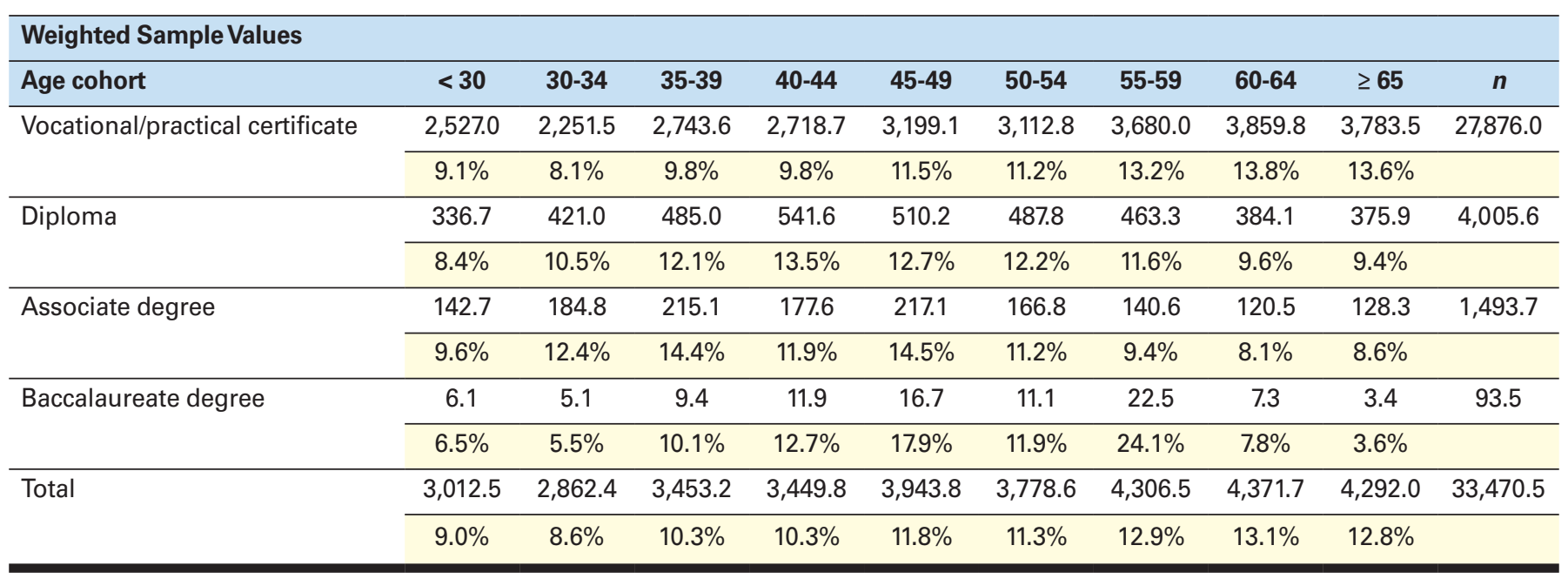

Highest level of nursing education

In terms of highest level of nursing education, $77.8 \%$ of respondents indicated that a vocational/practical certificate in nursing was their highest level of nursing education. This represents a slight increase of 1.8 percentage points from 2015 (76.0\%). See Table 50.

\section{TABLE 50}

\section{Highest Level of Nursing Education-LPN/LVN}

\begin{tabular}{|c|c|c|c|c|}
\hline \multicolumn{5}{|l|}{ Weighted Sample Values } \\
\hline & \multicolumn{2}{|c|}{$2015(n=25,626.5)$} & \multicolumn{2}{|c|}{$2017(n=34,208.6)$} \\
\hline & $n$ & Percent & $n$ & Percent \\
\hline Diploma & $3,882.5$ & $15.2 \%$ & $4,900.8$ & $14.3 \%$ \\
\hline Associate degree & $1,888.6$ & $7.4 \%$ & $2,509.6$ & $7.3 \%$ \\
\hline Baccalaureate degree & 308.5 & $1.2 \%$ & 182.8 & $0.5 \%$ \\
\hline
\end{tabular}


Vocational/practical certificate

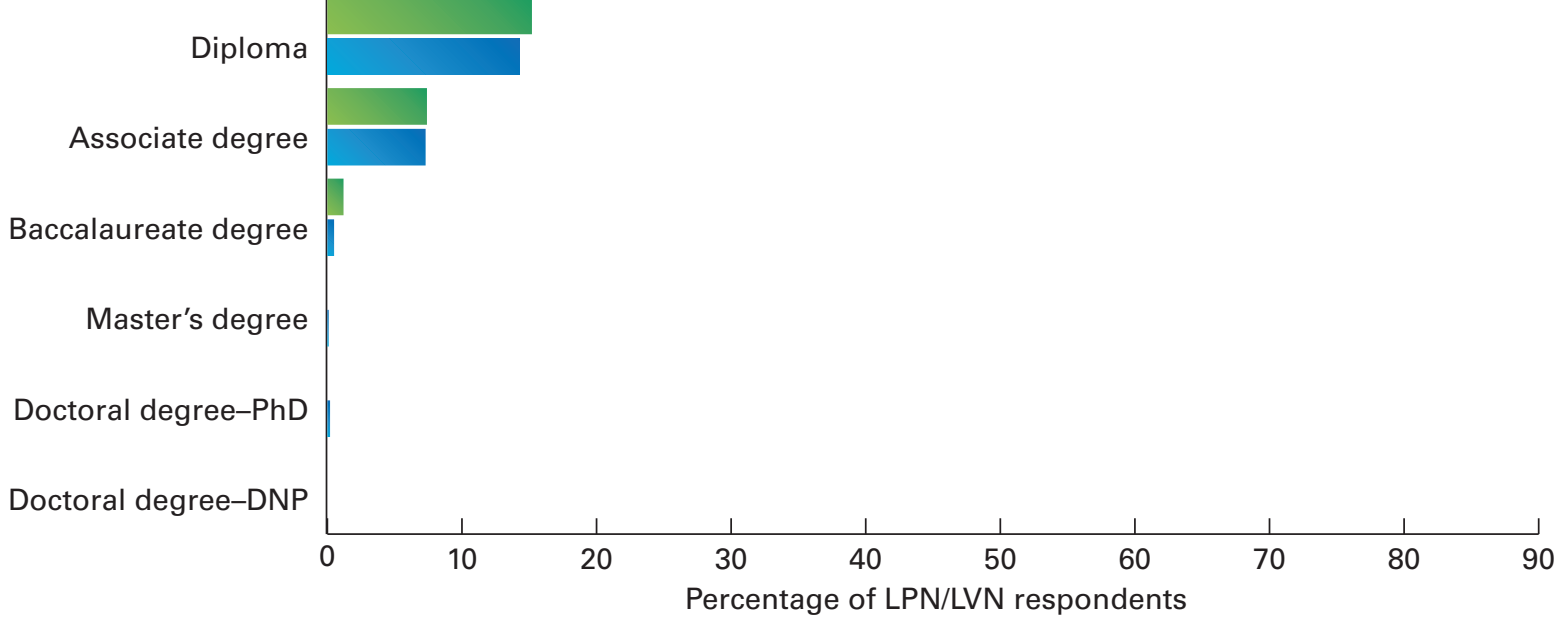

Note. In the 2013 and 2015 surveys, a single question "What is your highest level of education?" was asked, with the set of possible responses including both nursing and non-nursing degrees. In 2017, two separate questions were asked, "Highest level of nursing education" and "Highest level of nonnursing education".

Highest level of nursing education by race. Across nearly all race/ethnicity groups the most common highest level of education is the vocational/practical certificate. Asian nurses are substantially more likely to have a baccalaureate degree as their highest education as compared to other groups ( $8.1 \%$ versus $0.5 \%$ overall). See Table 51.

\section{TABLE 51}

\section{Highest Level of Nursing Education by Race-LPN/LVN}

\begin{tabular}{|c|c|c|c|c|c|}
\hline \multicolumn{6}{|l|}{ Weighted Sample Values } \\
\hline & $\begin{array}{c}\text { Vocational/Practical } \\
\text { Certificate }\end{array}$ & Diploma & $\begin{array}{l}\text { Associate } \\
\text { Degree }\end{array}$ & $\begin{array}{l}\text { Baccalaureate } \\
\text { Degree }\end{array}$ & $n$ \\
\hline \multirow[t]{2}{*}{ American Indian or Alaska Native } & 136.7 & 24.7 & 27.3 & 0.7 & 189.4 \\
\hline & $72.2 \%$ & $13.0 \%$ & $14.4 \%$ & $0.4 \%$ & \\
\hline \multirow[t]{2}{*}{ Asian } & 539.4 & 146.3 & 111.3 & 71.0 & 871.6 \\
\hline & $61.9 \%$ & $16.8 \%$ & $12.8 \%$ & $8.1 \%$ & \\
\hline \multirow[t]{2}{*}{ Native Hawaiian or other Pacific Islander } & 38.7 & 8.8 & 3.2 & 1.6 & 52.2 \\
\hline & $74.0 \%$ & $16.8 \%$ & $6.1 \%$ & $3.1 \%$ & \\
\hline \multirow[t]{2}{*}{ White/Caucasian } & $18,254.9$ & $3,062.3$ & $1,539.6$ & 57.5 & $22,917.1$ \\
\hline & $79.7 \%$ & $13.4 \%$ & $6.7 \%$ & $0.3 \%$ & \\
\hline \multirow[t]{2}{*}{ Two or more races } & 458.9 & 120.3 & 47.1 & 3.3 & 629.7 \\
\hline & $72.9 \%$ & $19.1 \%$ & $7.5 \%$ & $0.5 \%$ & \\
\hline \multirow[t]{2}{*}{ Total } & $26,362.0$ & $4,847.6$ & $2,489.6$ & 181.5 & $33,890.4$ \\
\hline & $77.8 \%$ & $14.3 \%$ & $7.3 \%$ & $0.5 \%$ & \\
\hline
\end{tabular}

Note. In the 2013 and 2015 surveys, a single question "What is your highest level of education?" was asked, with the set of possible responses including both nursing and non-nursing degrees. n 2017, two separate questions were asked, "Highest level of nursing education" and "Highest level of nonnursing education". 
Almost a quarter of responding LPNs/LVNs indicated they had a non-nursing degree. Of those respondents, $68.8 \%$ reported an associate level non-nursing degree, $25.0 \%$ reported a baccalaureate level non-nursing degree, and an additional $6.2 \%$ reported a graduate level non-nursing degree. See Table 52.

TABLE 52

\section{Highest Level of Non-Nursing Education-LPN/LVN}

\begin{tabular}{lcr}
\hline Weighted Sample Values & & \multicolumn{2}{c}{$\mathbf{2 0 1 7}(\boldsymbol{n}=\mathbf{9 , 8 3 2 . 6 )}$} \\
\hline & $\boldsymbol{n}$ & Percent \\
\hline Associate degree & $6,762.0$ & $68.8 \%$ \\
\hline Baccalaureate degree & $2,460.1$ & $25.0 \%$ \\
\hline Master's degree & 515.6 & $5.2 \%$ \\
\hline Doctoral degree & 95.0 & $1.0 \%$ \\
\hline
\end{tabular}

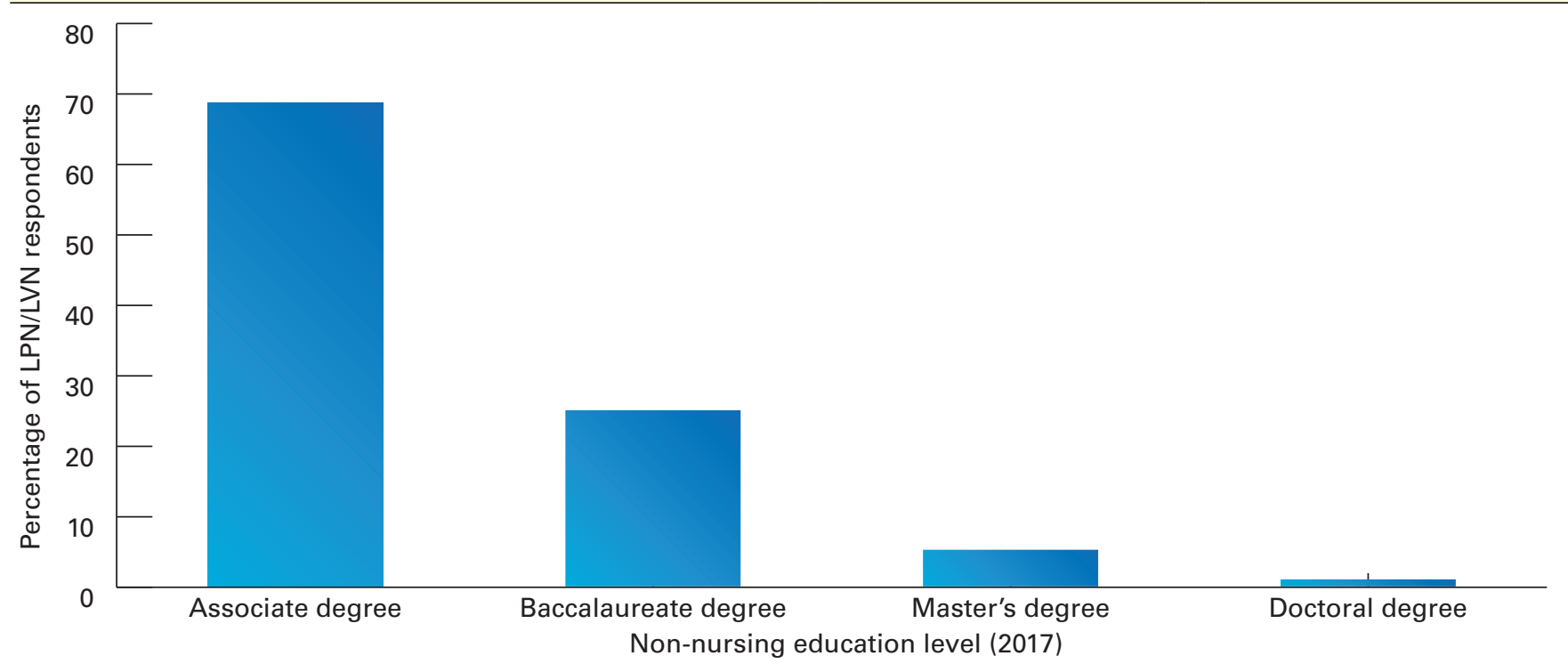

Note. In the 2013 and 2015 surveys, a single question "What is your highest level of education?" was asked, with the set of possible responses including both nursing and non-nursing degrees. In 2017, two separate questions were asked, "Highest level of nursing education" and "Highest level of nonnursing education".

\section{Licensure}

\section{Number of years licensed}

LPN/LVN respondents to the 2017 survey were licensed for a median 19 years. Almost $40.7 \%$ of 2017 respondents were licensed for 10 years or less compared to $43.7 \%$ in 2015 . An additional $19.8 \%$ were licensed between 11 and 20 years, which is consistent with the $20.1 \%$ who were licensed as long in 2015. See Table 53.

\section{TABLE 53}

\section{Number of Years Licensed-LPN/LVN}

\begin{tabular}{|c|c|c|c|c|}
\hline \multicolumn{5}{|c|}{ Weighted Sample Values } \\
\hline & \multicolumn{2}{|c|}{$2015(n=26,138.0)$} & \multicolumn{2}{|c|}{$2017(n=33,652.6)$} \\
\hline & $n$ & Percent & $n$ & Percent \\
\hline $0-10$ years & $11,417.8$ & $43.7 \%$ & $13,694.1$ & $40.7 \%$ \\
\hline $11-20$ years & $5,258.9$ & $20.1 \%$ & $6,674.0$ & $19.8 \%$ \\
\hline $21-30$ years & $4,018.0$ & $15.4 \%$ & $5,483.9$ & $16.3 \%$ \\
\hline $31-40$ years & $3,552.0$ & $13.6 \%$ & $4,531.9$ & $13.5 \%$ \\
\hline
\end{tabular}




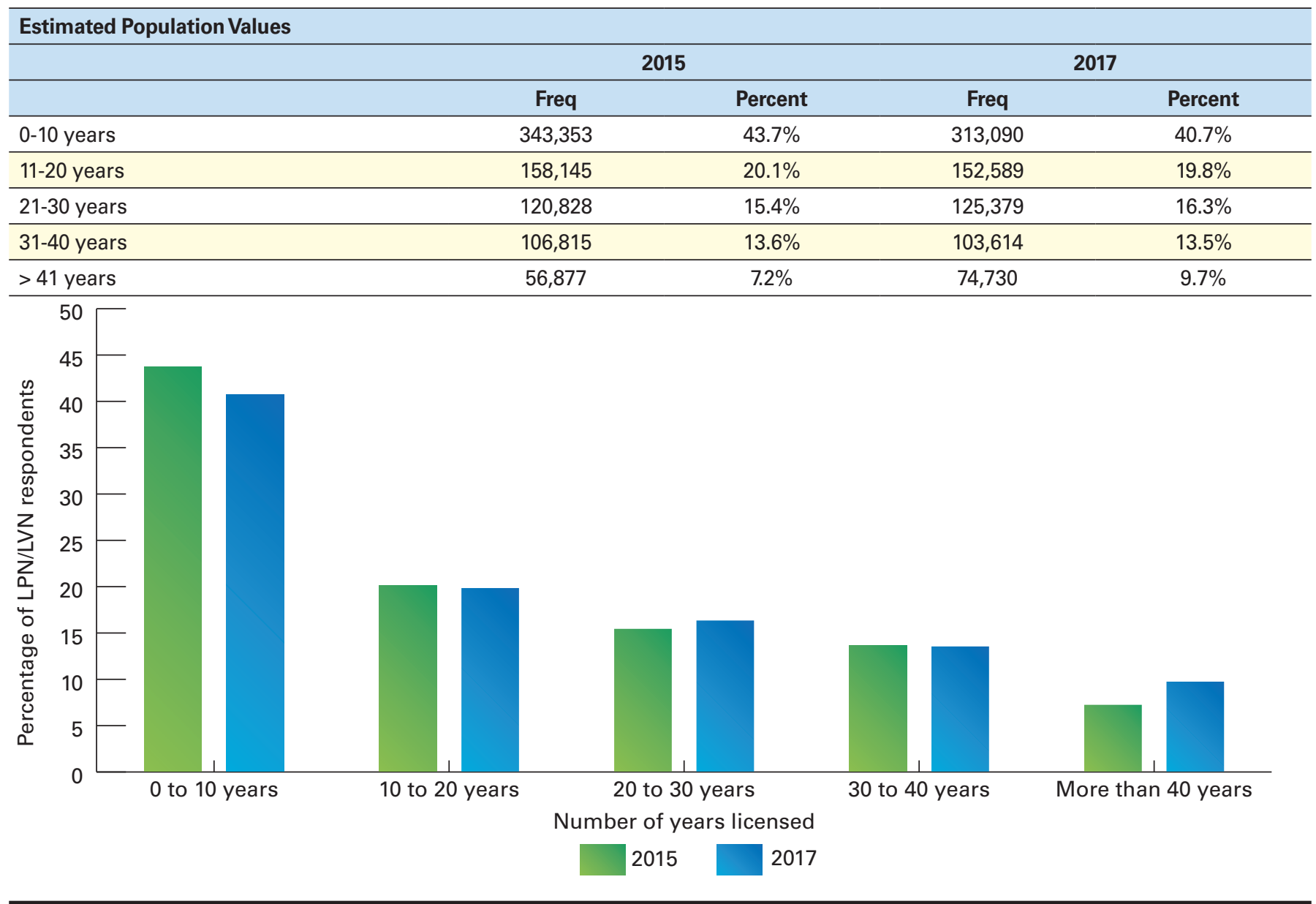

Initially licensed in the United States

In this survey, $97.5 \%$ of LPN/LVN respondents were initially licensed in the United States. This is consistent with results from 2015 (98.5\%). See Table 54.

\section{TABLE 54}

\section{Initially Licensed in the United States - LPN/LVN}

\begin{tabular}{|c|c|c|c|c|}
\hline \multicolumn{5}{|c|}{ Weighted Sample Values } \\
\hline & \multicolumn{2}{|c|}{$2015(n=30,179.4)$} & \multicolumn{2}{|c|}{$2017(n=34,598.2)$} \\
\hline & $n$ & Percent & $n$ & Percent \\
\hline Yes & $29,731.6$ & $98.5 \%$ & $33,719.3$ & $97.5 \%$ \\
\hline & Freq & Percent & Freq & Percent \\
\hline No & 13,466 & $1.5 \%$ & 20,095 & $2.5 \%$ \\
\hline Yes & 894,084 & $98.5 \%$ & 770,929 & $97.5 \%$ \\
\hline
\end{tabular}

Note. In the 2015 survey, respondents were asked “In what country were you initially licensed as an RN or LPN?" 


\section{Employment}

Employment status

Among responding LPNs/LVNs, $65.0 \%$ reported being actively employed in nursing full time, which is an increase over 2 years ago. The most notable decrease was among those who selected unemployed, seeking work as a nurse, which decreased from $5.1 \%$ in 2015 to $3.4 \%$ in 2017 . See Table 55 .

\section{TABLE 55}

\section{Employment Status-LPN/LVN}

\begin{tabular}{|c|c|c|c|c|}
\hline \multicolumn{5}{|l|}{ Weighted Sample Values } \\
\hline & \multicolumn{2}{|c|}{$2015(n=30,766.0)$} & \multicolumn{2}{|c|}{$2017(n=34,570.2)$} \\
\hline & $n$ & Percent & $n$ & Percent \\
\hline Actively employed in nursing part time & $3,714.0$ & $12.1 \%$ & $4,151.9$ & $12.0 \%$ \\
\hline Actively employed in nursing per diem & $2,179.4$ & $7.1 \%$ & $2,227.5$ & $6.4 \%$ \\
\hline Actively employed, non-nursing part time & 868.5 & $2.8 \%$ & 756.7 & $2.2 \%$ \\
\hline Actively employed, non-nursing per diem & 386.7 & $1.3 \%$ & 193.6 & $0.6 \%$ \\
\hline Working in nursing as a volunteer & 366.1 & $1.2 \%$ & 408.9 & $1.2 \%$ \\
\hline Unemployed, seeking work as a nurse & $1,558.7$ & $5.1 \%$ & $1,162.0$ & $3.4 \%$ \\
\hline Unemployed, not seeking work as a nurse & $1,588.9$ & $5.2 \%$ & $1,595.0$ & $4.6 \%$ \\
\hline & \multicolumn{2}{|c|}{2015} & \multicolumn{2}{|c|}{2017} \\
\hline & Freq & Percent & Freq & Percent \\
\hline Actively employed in nursing full time & 566,053 & $61.2 \%$ & 513,884 & $65.0 \%$ \\
\hline Actively employed in nursing part time & 111,686 & $12.1 \%$ & 94,925 & $12.0 \%$ \\
\hline Actively employed in nursing per diem & 65,540 & $7.1 \%$ & 50,928 & $6.4 \%$ \\
\hline Actively employed, non-nursing full time & 45,236 & $4.9 \%$ & 29,874 & $3.8 \%$ \\
\hline Actively employed, non-nursing part time & 26,116 & $2.8 \%$ & 17,301 & $2.2 \%$ \\
\hline Actively employed, non-nursing per diem & 11,629 & $1.3 \%$ & 4,427 & $0.6 \%$ \\
\hline
\end{tabular}




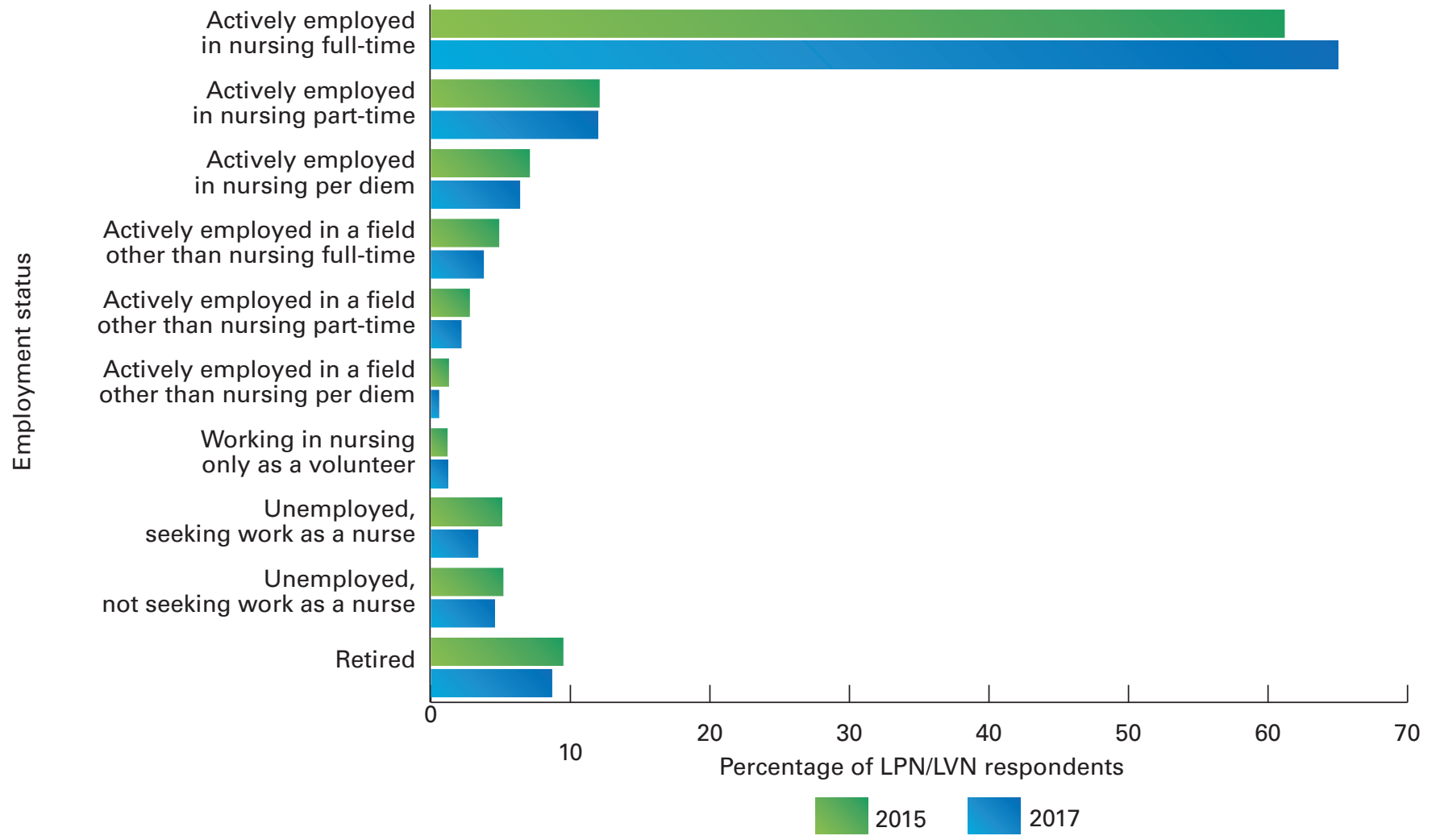

Note. Respondents were asked to select all that apply.

\section{Reasons for being unemployed}

Respondents were asked to select all the applicable reasons for being unemployed. Taking care of home and family was the most frequently selected reason for being unemployed; $41.4 \%$ of LPN/LVN respondents selected this reason in 2017 , compared to $39.1 \%$ in 2015 . The second and third most frequently selected reasons were other by $26.4 \%$ of respondents followed by disabled (21.1\%). By comparison, the second most frequently selected reason in 2015 was difficulty in finding a nursing position (23.1\%) followed by other (22.7\%). See Table 56.

\section{TABLE 56}

\section{Reasons for Being Unemployed-LPN/LVN}

\begin{tabular}{|c|c|c|c|c|}
\hline \multicolumn{5}{|l|}{ Weighted Sample Values } \\
\hline & \multicolumn{2}{|c|}{$2015(n=2,644.5)$} & \multicolumn{2}{|c|}{$2017(n=2,696.8)$} \\
\hline & $n$ & Percent & $n$ & Percent \\
\hline Disabled & 463.3 & $17.5 \%$ & 570.0 & $21.1 \%$ \\
\hline Inadequate salary & 77.9 & $3.0 \%$ & 111.5 & $4.1 \%$ \\
\hline Difficulty in finding a nursing position & 610.4 & $23.1 \%$ & 419.6 & $15.6 \%$ \\
\hline Other & 600.1 & $22.7 \%$ & 713.0 & $26.4 \%$ \\
\hline
\end{tabular}


Taking care of home and family

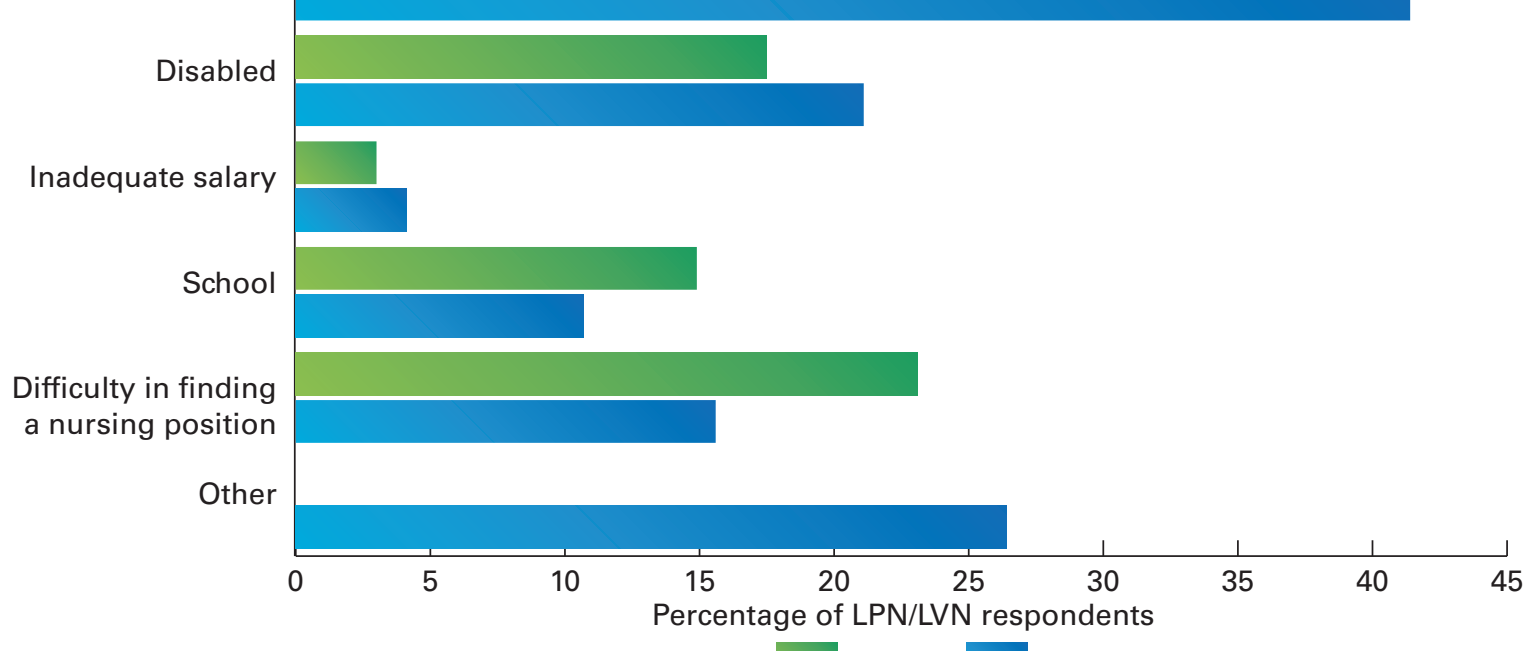

2015

2017

Note. Respondents were asked to select all that apply.

\section{Number of positions currently held}

Respondents were asked to identify the number of positions in which they were currently employed as a nurse. In $2017,82.4 \%$ of LPN/ LVN respondents indicated holding just one position as a nurse compared to $84.5 \%$ in 2015 . The percentage of nurses who reported working in two positions increased from $13.4 \%$ in 2015 to $14.9 \%$ in 2017 . The percentage of respondents who indicated that they held three or more positions in nursing also increased slightly from $2.1 \%$ in 2015 to $2.7 \%$ in 2017 . See Table 57.

\section{TABLE 57}

\section{Number of Positions Currently Held-LPN/LVN}

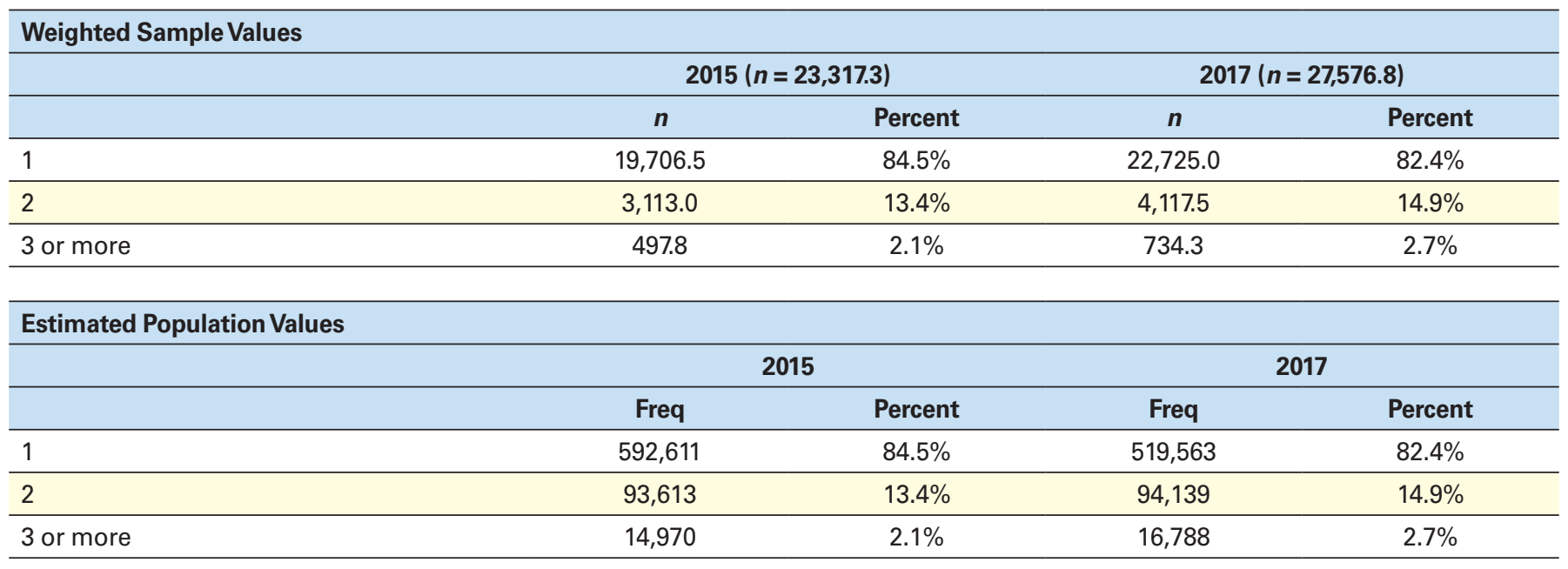

Note. Survey participants were asked to answer this question only if they were actively employed in nursing.

\section{Number of hours worked during a typical week in all nursing positions}

In this survey, $59.4 \%$ of responding LPNs/LVNs reported working 32 to 40 hours in a typical week in all positions, representing a decrease of less than one percentage point. The second most frequently reported category was 41 to 50 hours (16.0\%). This represents a slight increase from 2015 (15.2\%). See Table 58. 


\section{TABLE 58}

\section{Number of Hours Worked During a Typical Week in All Nursing Positions - LPN/LVN}

\begin{tabular}{|c|c|c|c|c|}
\hline \multicolumn{5}{|c|}{ Weighted Sample Values } \\
\hline & \multicolumn{2}{|c|}{$2015(n=22,450.6)$} & \multicolumn{2}{|c|}{$2017(n=27,505.7)$} \\
\hline & $n$ & Percent & $n$ & Percent \\
\hline $16-23$ & $1,088.9$ & $4.9 \%$ & $1,298.4$ & $4.7 \%$ \\
\hline 24-31 & $1,866.4$ & $8.3 \%$ & $2,031.1$ & $7.4 \%$ \\
\hline $41-50$ & $3,410.0$ & $15.2 \%$ & $4,412.7$ & $16.0 \%$ \\
\hline $51-60$ & 928.3 & $4.1 \%$ & $1,391.1$ & $5.1 \%$ \\
\hline 61 or more & 681.1 & $3.0 \%$ & 911.5 & $3.3 \%$ \\
\hline \multicolumn{5}{|c|}{ Estimated Population Values } \\
\hline $16-23$ & 32,744 & $4.9 \%$ & 29,686 & $4.7 \%$ \\
\hline $24-31$ & 56,126 & $8.3 \%$ & 46,437 & $7.4 \%$ \\
\hline $32-40$ & 407,859 & $60.4 \%$ & 373,314 & $59.4 \%$ \\
\hline $41-50$ & 102,546 & $15.2 \%$ & 100,888 & $16.0 \%$ \\
\hline $51-60$ & 27,916 & $4.1 \%$ & 31,805 & $5.1 \%$ \\
\hline 61 or more & 20,482 & $3.0 \%$ & 20,841 & $3.3 \%$ \\
\hline
\end{tabular}

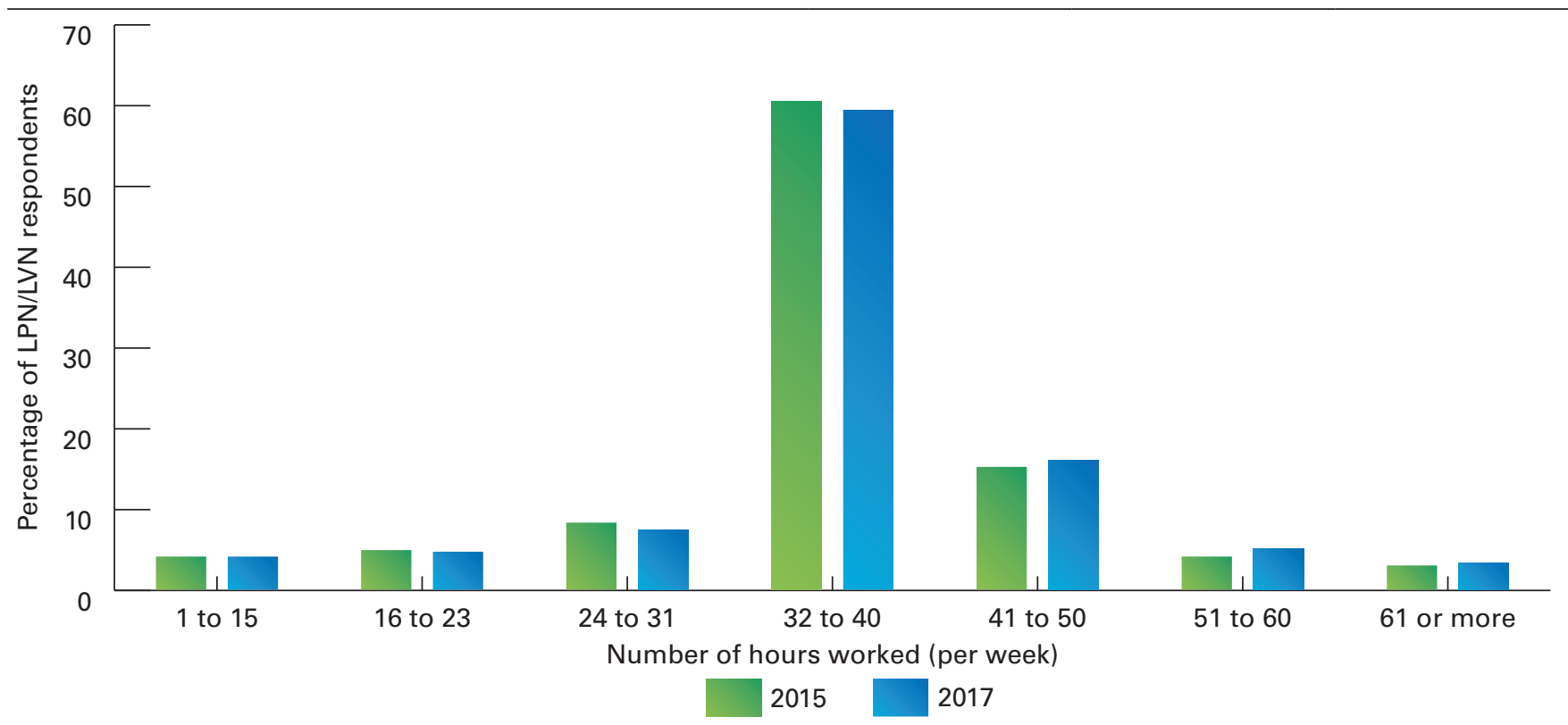

Note. Survey participants were asked to answer this question only if they were actively employed in nursing.

\section{Primary nursing practice position setting}

In the 2017 survey, response options related to primary and secondary practice setting were slightly modified. Hospice and dialysis center were added as new categories. Of those LPNs/LVNs who responded to the question, $31.7 \%$ indicated that their primary nursing practice position was in a nursing home/extended care setting. This represents an increase of 1.6 percentage points from 2015 . Home health was 
the second most frequently selected setting by $14 \%$ of LPNs/LVNs, followed by ambulatory care selected by $6.8 \%$. The percentage of respondents who selected other increased from $14.9 \%$ in 2015 to $17.4 \%$ in 2017. See Table 59.

\section{TABLE 59}

\section{Primary Nursing Practice Position Setting-LPN/LVN}

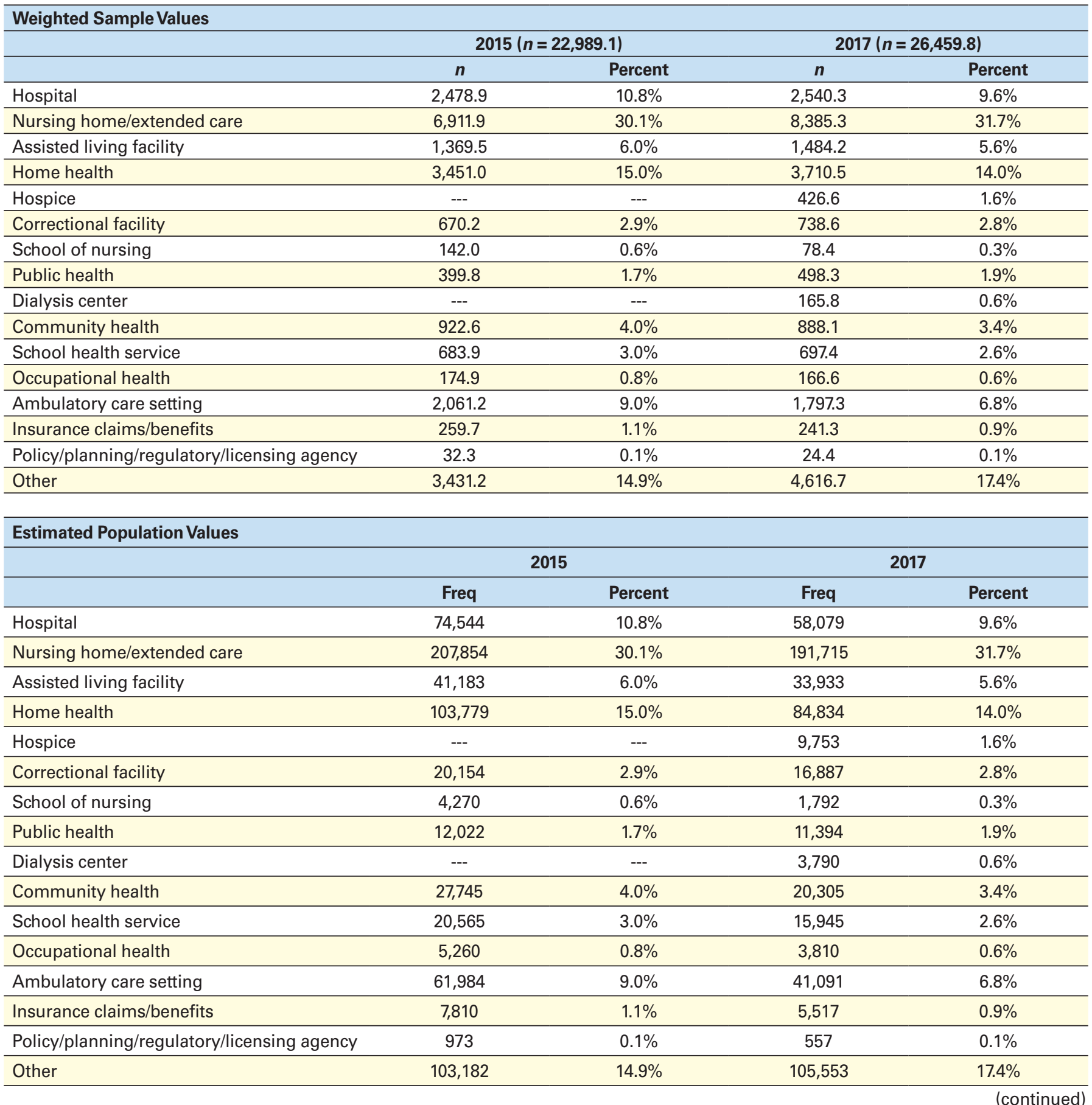




\section{Primary Nursing Practice Position Setting-LPN/LVN (continued)}

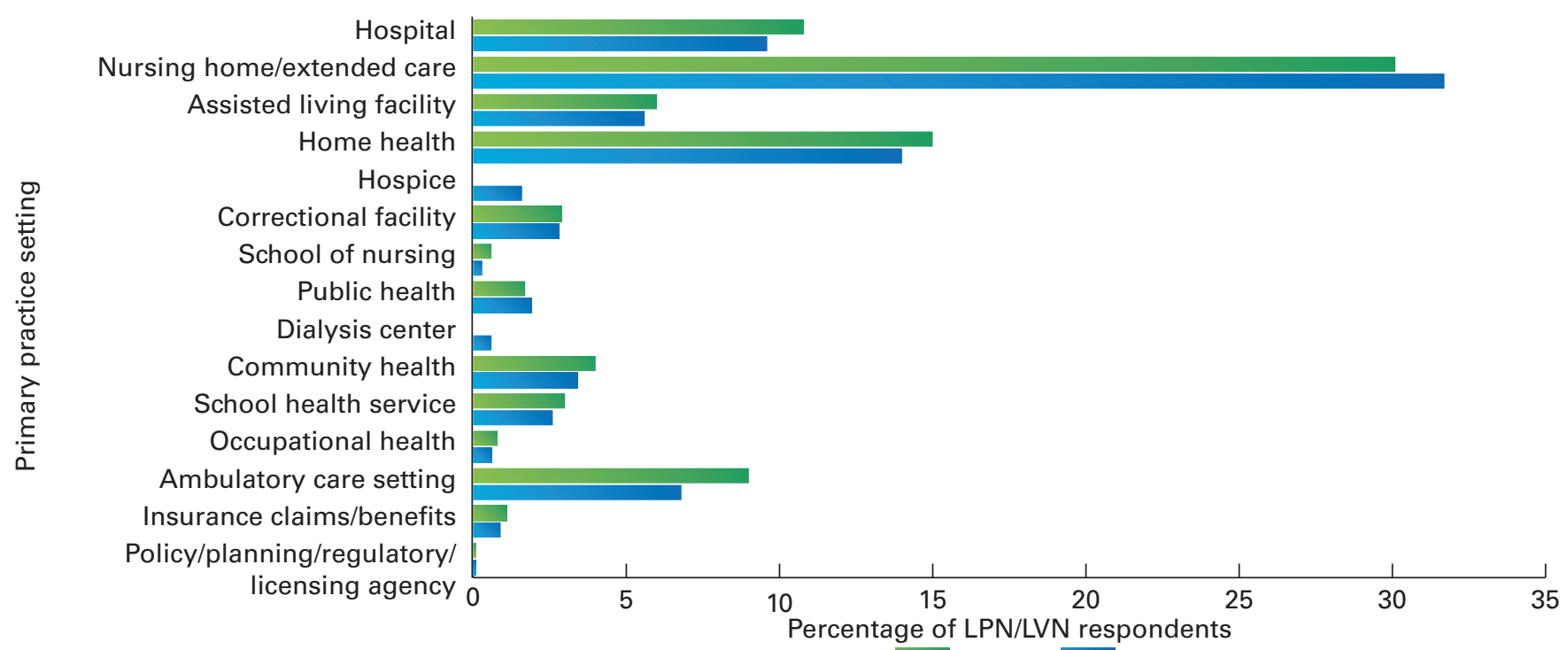

Note. Survey participants were asked to answer this question only if they were actively employed in nursing.

\section{Primary nursing position title}

In $2017,73.1 \%$ of LPN/LVN respondents indicated that the title that most closely corresponds to their primary nursing position was that of staff nurse, an increase of 4.3 percentage points from 2015. The second most frequently selected position title was other-healthrelated. The proportion of respondents who selected other-health-related increased from $14.6 \%$ in 2015 to $16.0 \%$ in 2017 . A higher proportion of LPNs/LVNs selected nurse manager in 2017 (6.2\%) than in 2015 (5.8\%). See Table 60.

TABLE 60

\section{Primary Nursing Position Title-LPN/LVN}

\begin{tabular}{|c|c|c|c|c|}
\hline \multicolumn{5}{|l|}{ Weighted Sample Values } \\
\hline & \multicolumn{2}{|c|}{$2015(n=23,567.8)$} & \multicolumn{2}{|c|}{$2017(n=26,776.9)$} \\
\hline & $n$ & Percent & $n$ & Percent \\
\hline Nurse researcher & 65.0 & $0.3 \%$ & 51.2 & $0.2 \%$ \\
\hline Nurse executive & 137.6 & $0.6 \%$ & 70.9 & $0.3 \%$ \\
\hline Nurse faculty/educator & 967.9 & $4.1 \%$ & 257.5 & $1.0 \%$ \\
\hline Advanced practice registered nurse & 401.7 & $1.7 \%$ & 9.0 & $0.0 \%$ \\
\hline Staff nurse & $16,214.1$ & $68.8 \%$ & $19,564.6$ & $73.1 \%$ \\
\hline Case manager & 595.3 & $2.5 \%$ & 561.4 & $2.1 \%$ \\
\hline Other-health related & $3,444.2$ & $14.6 \%$ & $4,275.5$ & $16.0 \%$ \\
\hline
\end{tabular}




\begin{tabular}{|c|c|c|c|c|}
\hline \multicolumn{5}{|l|}{ Estimated Population Values } \\
\hline & \multicolumn{2}{|c|}{2015} & \multicolumn{2}{|c|}{2017} \\
\hline & Freq & Percent & Freq & Percent \\
\hline Nurse researcher & 1,955 & $0.3 \%$ & 1,170 & $0.2 \%$ \\
\hline Nurse executive & 4,138 & $0.6 \%$ & 1,621 & $0.3 \%$ \\
\hline Nurse faculty/educator & 29,107 & $4.1 \%$ & 5,887 & $1.0 \%$ \\
\hline Advanced practice registered nurse & 12,079 & $1.7 \%$ & 206 & $0.0 \%$ \\
\hline Staff nurse & 487,589 & $68.8 \%$ & 447,308 & $73.1 \%$ \\
\hline Case manager & 17,902 & $2.5 \%$ & 12,835 & $2.1 \%$ \\
\hline Other-health related & 103,572 & $14.6 \%$ & 97,751 & $16.0 \%$ \\
\hline
\end{tabular}

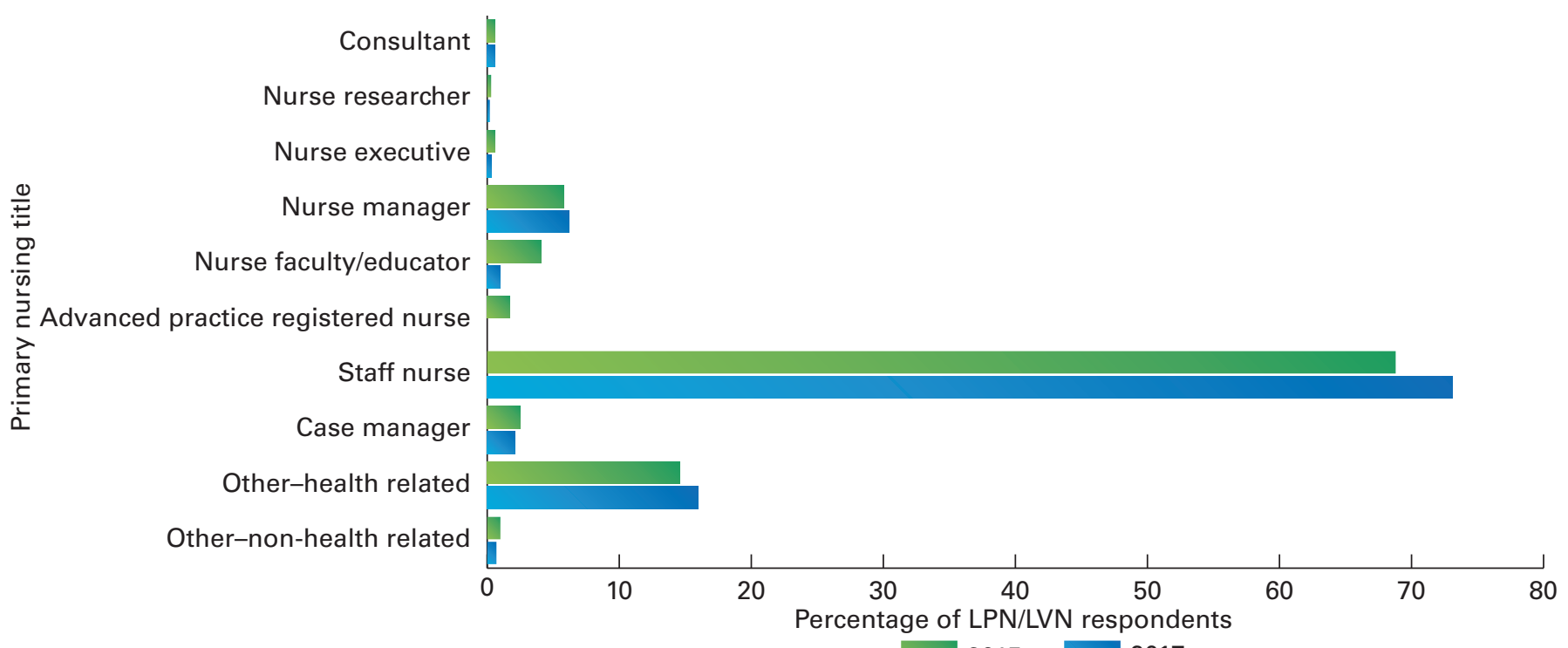

Note. Survey participants were asked to answer this question only if they were actively employed in nursing.

\section{Primary nursing position speciality}

Response options related to primary and secondary practice specialty were modified from the 2015 instrument. Adult health and family health were separated into two categories; cardiology was added as a category; and other was separated into other-clinical and other-nonclinical. Primary care, genetics, informatics, neurology/neurosurgical, orthopedic, radiology, urology, and telehealth were all removed from the list of categories.

Of LPN/LVN respondents, 30.5\% selected geriatrics as the employment specialty that most closely corresponded to their primary nursing practice position. The second most frequently selected employment specialty was home health (8.8\%) followed by adult health (7.8\%). A smaller proportion of LPNs/LVNs selected other in 2017 (12.1\%) compared to 2015 (15.5\%). The 12.1\% who comprised other in 2017 included the $10.8 \%$ of LPN/LVN respondents who indicated other-clinical specialties and $1.3 \%$ who indicated other-nonclinical specialties. See Table 61. 


\section{TABLE 61}

\section{Primary Nursing Position Specialty-LPN/LVN}

\begin{tabular}{|c|c|c|c|c|}
\hline \multicolumn{5}{|l|}{ Weighted Sample Values } \\
\hline & $2015(n=21,932.4)$ & & $2017(n=25,214.9)$ & \\
\hline & $n$ & Percent & $n$ & Percent \\
\hline Adult health & 960.6 & $4.4 \%$ & $1,968.0$ & $7.8 \%$ \\
\hline Family health & --- & --- & $1,712.4$ & $6.8 \%$ \\
\hline Cardiology & --- & --- & 250.4 & $1.0 \%$ \\
\hline Community & 262.6 & $1.2 \%$ & 216.8 & $0.9 \%$ \\
\hline Geriatric/gerontology & $6,064.1$ & $27.7 \%$ & $7,685.8$ & $30.5 \%$ \\
\hline Home health & $2,109.3$ & $9.6 \%$ & $2,228.2$ & $8.8 \%$ \\
\hline Maternal-child health/obstetrics & 120.5 & $0.6 \%$ & 225.1 & $0.9 \%$ \\
\hline Oncology & 137.1 & $0.6 \%$ & 152.9 & $0.6 \%$ \\
\hline Palliative care/hospice & 348.2 & $1.6 \%$ & 354.7 & $1.4 \%$ \\
\hline Pediatrics & $1,326.0$ & $6.1 \%$ & $1,880.2$ & $7.5 \%$ \\
\hline Neonatal & 28.2 & $0.1 \%$ & 28.8 & $0.1 \%$ \\
\hline Perioperative & 93.1 & $0.4 \%$ & 76.4 & $0.3 \%$ \\
\hline Public health & 173.1 & $0.8 \%$ & 257.6 & $1.0 \%$ \\
\hline Psychiatric/mental health/substance abuse & $1,084.8$ & $5.0 \%$ & $1,205.0$ & $4.8 \%$ \\
\hline Rehabilitation & 847.7 & $3.9 \%$ & $1,081.5$ & $4.3 \%$ \\
\hline Primary care & $1,695.5$ & $7.7 \%$ & --- & --- \\
\hline Genetics & 182.2 & $0.8 \%$ & --- & --- \\
\hline Informatics & 41.4 & $0.2 \%$ & -- & --- \\
\hline Neurology/neurosurgical & 90.6 & $0.4 \%$ & -- & --- \\
\hline Orthopedic & 185.5 & $0.9 \%$ & -- & --- \\
\hline Radiology & 24.2 & $0.1 \%$ & --- & --- \\
\hline Urology & 102.1 & $0.5 \%$ & --- & --- \\
\hline
\end{tabular}




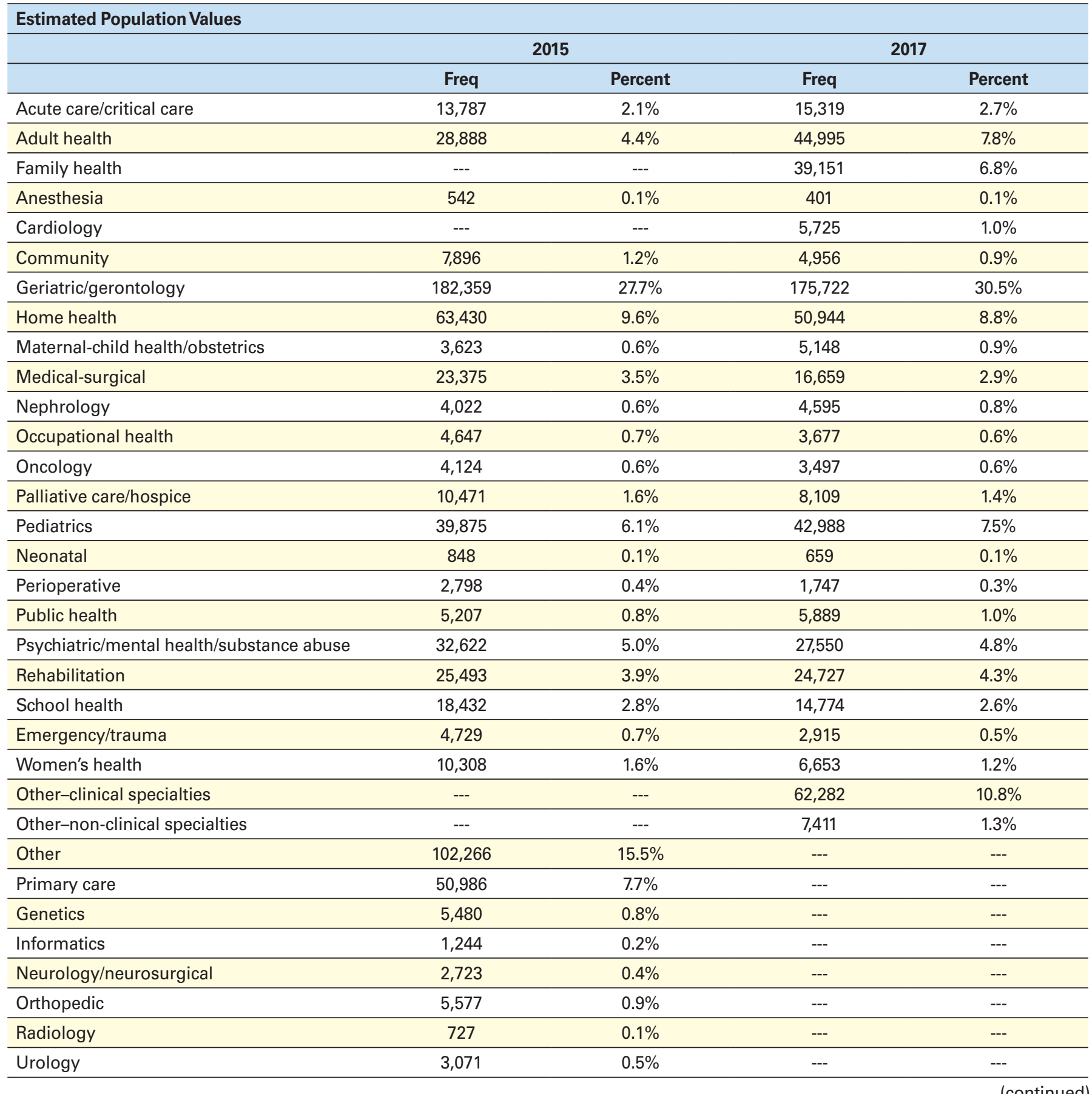




\section{Primary Nursing Position Specialty-LPN/LVN (continued)}

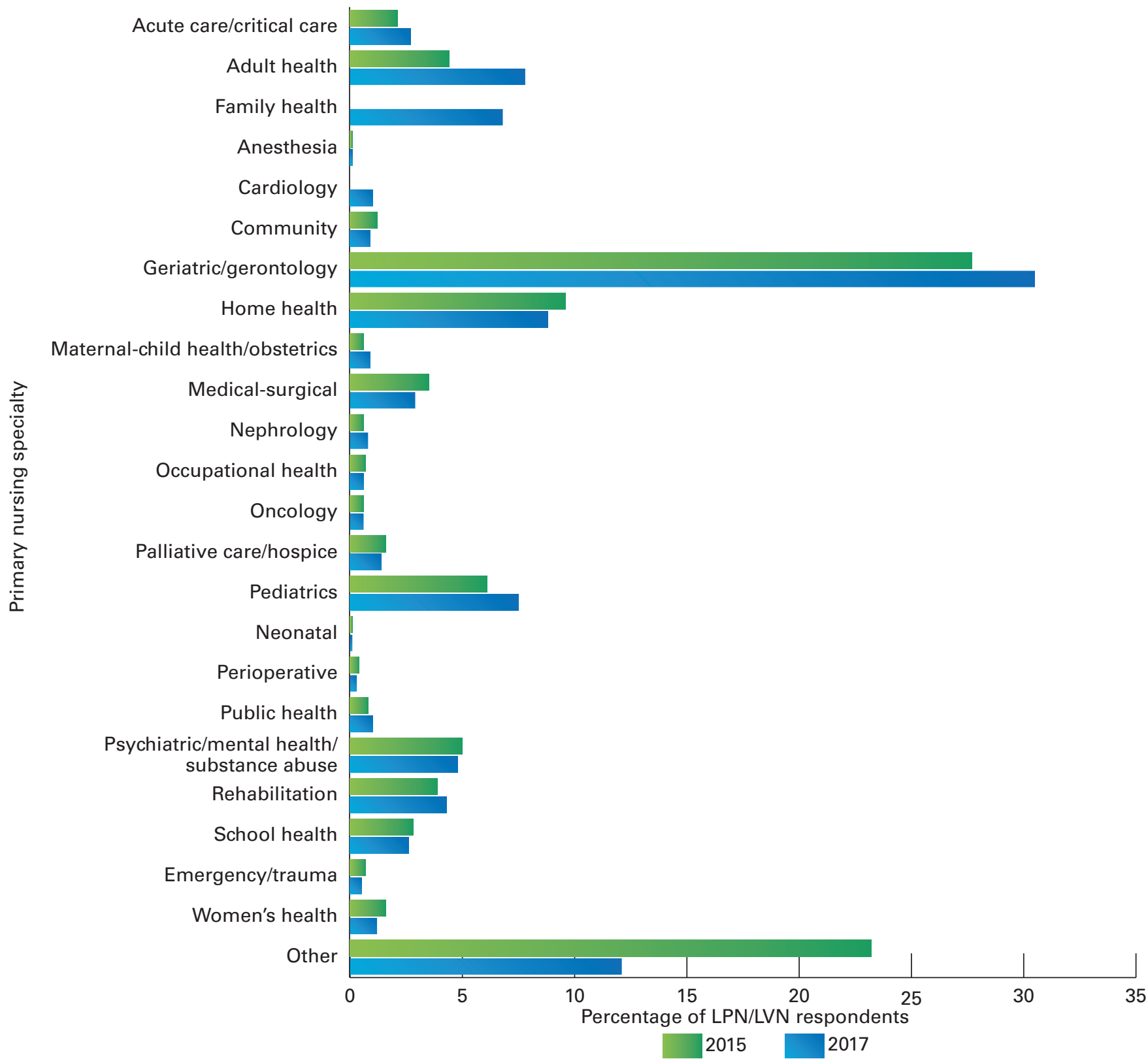

Note. Survey participants were asked to answer this question only if they were actively employed in nursing.

\section{Secondary nursing position setting}

Nursing home/extended care settings was the most frequently chosen setting by $32.5 \%$ of LPN/LVN respondents as their secondary nursing position This represents 5.6 percentage point increase from 2015. At the same time, the percentage of LPNs/LVNs who indicated their secondary nursing position was in a home health setting decreased by 5.1 percentage points from $31.9 \%$ in 2015 to $26.8 \%$ in 2017. The percentage that identified an assisted living facility to be their secondary nursing position setting saw little change between the two surveys. See Table 62. 


\section{TABLE 62}

\section{Secondary Nursing Position Setting-LPN/LVN}

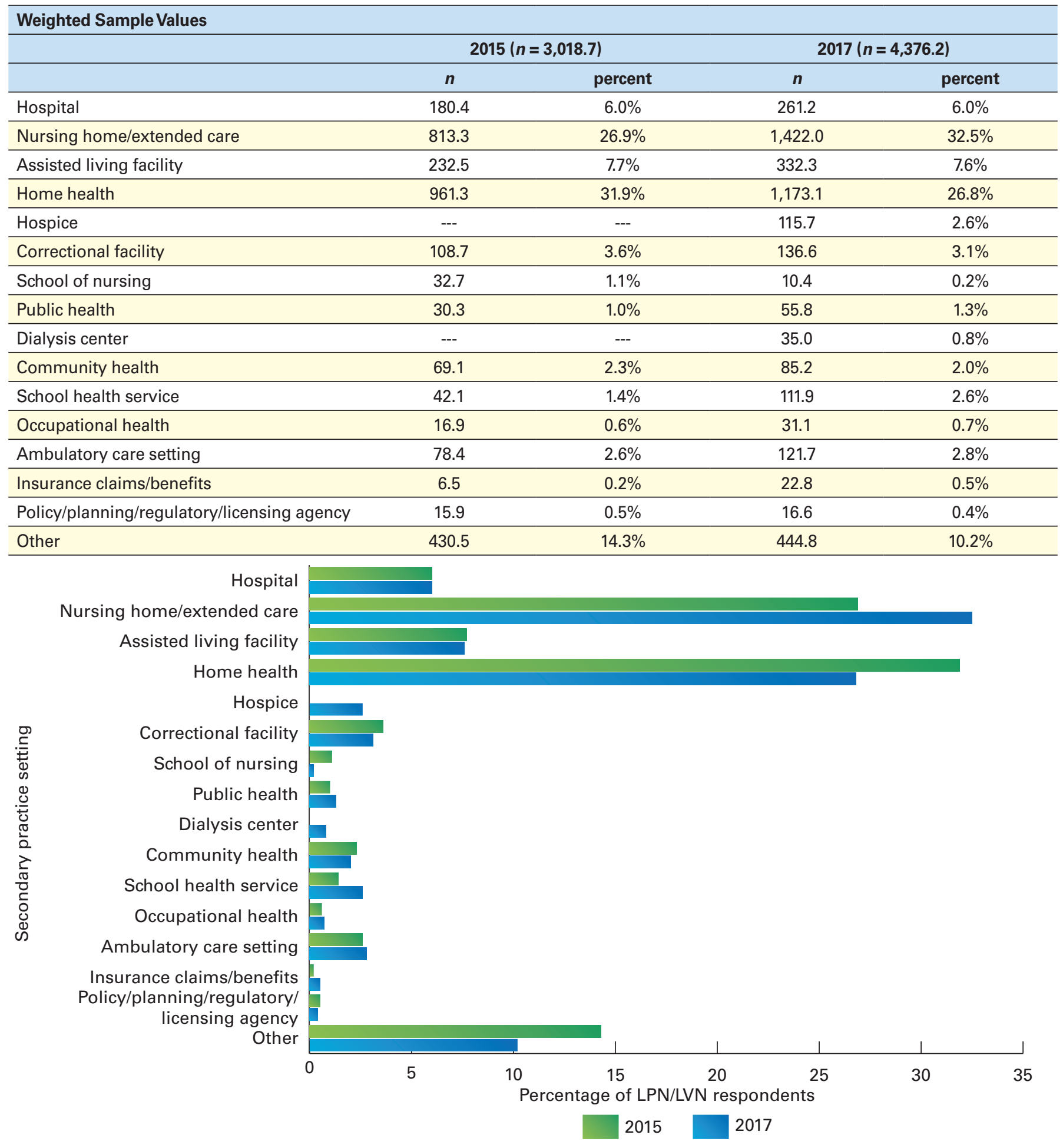




\section{Secondary nursing position title}

Over three-quarters of LPN/LVN respondents reported their secondary nursing position title as staff nurse. This represents an increase of 5.8 percentage points between 2015 and 2017. The percentage of LPNs/LVNs who reported a secondary nursing position title of nurse manager increased by 1.4 percentage points from 2015 to 2017 . The percentage of LPN/LVN respondents who were nurse faculty/educators in their secondary nursing position decreased from $4.4 \%$ in 2015 to $1.4 \%$ in 2017 . See Table 63.

\section{TABLE 63}

\section{Secondary Nursing Position Title-LPN/LVN}

\begin{tabular}{|c|c|c|c|c|}
\hline \multicolumn{5}{|l|}{ Weighted Sample Values } \\
\hline & \multicolumn{2}{|c|}{$2015(n=2,980.3)$} & \multicolumn{2}{|c|}{$2017(n=4,217.2)$} \\
\hline & $n$ & Percent & $n$ & Percent \\
\hline Nurse researcher & 7.0 & $0.2 \%$ & 1.3 & $0.0 \%$ \\
\hline Nurse executive & 10.4 & $0.4 \%$ & 10.0 & $0.2 \%$ \\
\hline Nurse faculty/educator & 132.0 & $4.4 \%$ & 58.4 & $1.4 \%$ \\
\hline Advanced practice registered nurse & 34.1 & $1.1 \%$ & 2.6 & $0.1 \%$ \\
\hline Staff nurse & $2,167.2$ & $72.7 \%$ & $3,309.2$ & $78.5 \%$ \\
\hline Case manager & 53.8 & $1.8 \%$ & 66.6 & $1.6 \%$ \\
\hline Other-health related & 440.3 & $14.8 \%$ & 528.9 & $12.5 \%$ \\
\hline
\end{tabular}

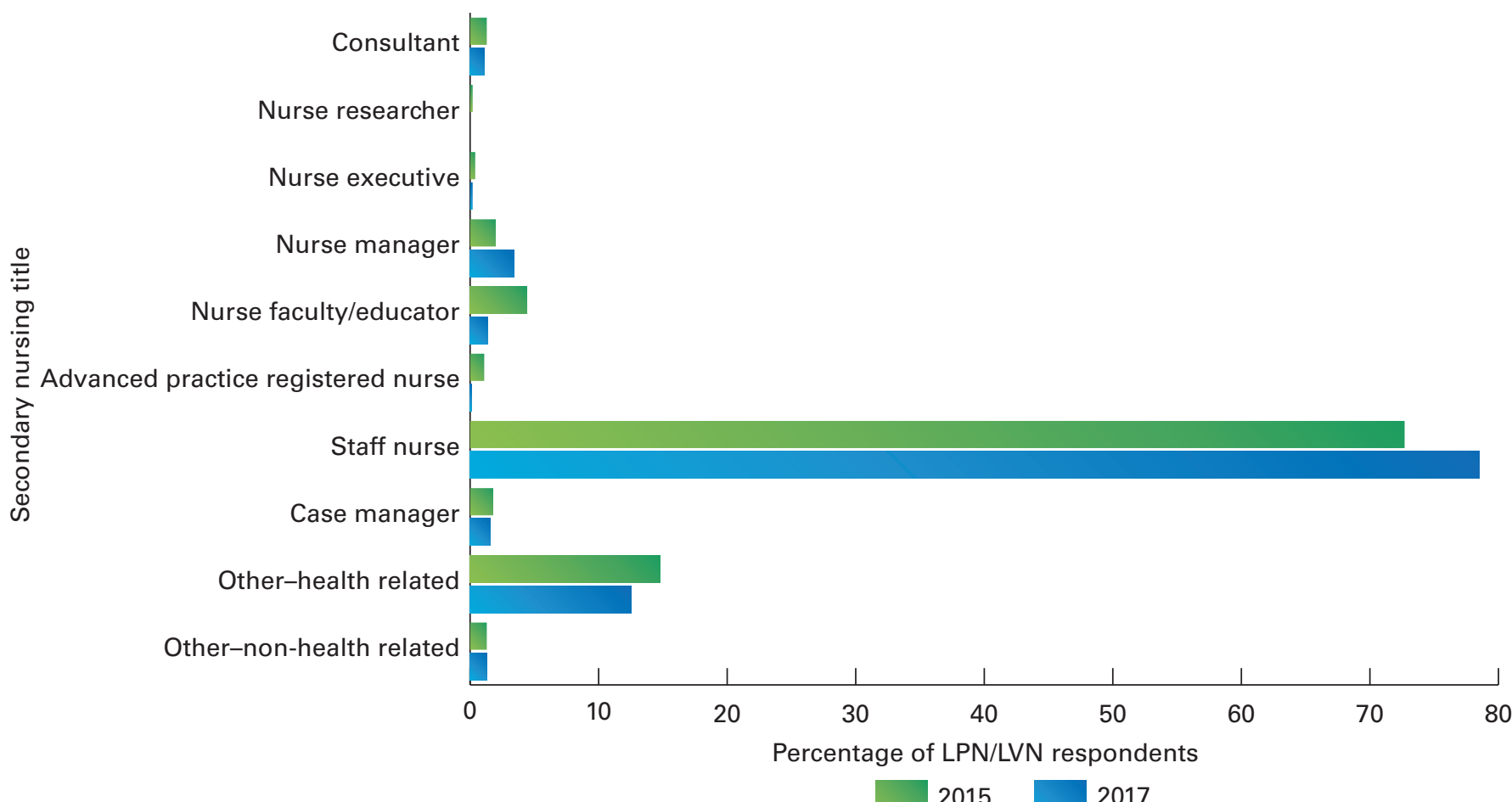

Note. Survey participants were asked to answer this question only if they were actively employed in nursing. 
Over one-third of LPNs/LVNs (34.5\%) reported their secondary nursing position specialty was geriatric/gerontology, an increase of 4.8 percentage points from 2015. Home health was the second most frequently selected specialty in both 2015 and 2017 , though there was a decrease of 3.3 percentage points. See Table 64.

\section{TABLE 64}

\section{Secondary Nursing Position Specialty－LPN/LVN}

\begin{tabular}{|c|c|c|c|c|}
\hline \multicolumn{5}{|l|}{ Weighted Sample Values } \\
\hline & \multicolumn{2}{|c|}{$2015(n=2,809.3)$} & \multicolumn{2}{|c|}{$2017(n=4,024.3)$} \\
\hline & $n$ & Percent & $n$ & Percent \\
\hline Adult health & 63.8 & $2.3 \%$ & 236.6 & $5.9 \%$ \\
\hline Family health & --- & --- & 88.6 & $2.2 \%$ \\
\hline Cardiology & --- & --- & 13.1 & $0.3 \%$ \\
\hline Community & 37.3 & $1.3 \%$ & 72.3 & $1.8 \%$ \\
\hline Geriatric/gerontology & 834.0 & $29.7 \%$ & $1,388.2$ & $34.5 \%$ \\
\hline Home health & 568.1 & $20.2 \%$ & 679.5 & $16.9 \%$ \\
\hline Occupational health & 17.2 & $0.6 \%$ & 27.3 & $0.7 \%$ \\
\hline Oncology & 1.6 & $0.1 \%$ & 9.0 & $0.2 \%$ \\
\hline Palliative care/hospice & 77.8 & $2.8 \%$ & 58.0 & $1.4 \%$ \\
\hline Pediatrics & 181.7 & $6.5 \%$ & 378.6 & $9.4 \%$ \\
\hline Neonatal & 8.4 & $0.3 \%$ & 1.7 & $0.0 \%$ \\
\hline Perioperative & 0.5 & $<0.1 \%$ & 2.9 & $0.1 \%$ \\
\hline Public health & 21.6 & $0.8 \%$ & 57.6 & $1.4 \%$ \\
\hline Psychiatric/mental health/substance abuse & 111.8 & $4.0 \%$ & 159.5 & $4.0 \%$ \\
\hline Other-Non-clinical specialties & --- & --- & 48.3 & $1.2 \%$ \\
\hline Other & 420.1 & $15.0 \%$ & -- & --- \\
\hline Primary care & 76.9 & $2.7 \%$ & --- & --- \\
\hline Genetics & 29.9 & $1.1 \%$ & -- & -- \\
\hline Informatics & 3.6 & $0.1 \%$ & --- & --- \\
\hline Neurology/neurosurgical & 3.4 & $0.1 \%$ & -- & -- \\
\hline Orthopedic & 6.7 & $0.2 \%$ & --- & --- \\
\hline Radiology & 1.8 & $0.1 \%$ & -- & -- \\
\hline Urology & 1.8 & $0.1 \%$ & --- & --- \\
\hline
\end{tabular}




\section{Secondary Nursing Position Specialty-LPN/LVN (continued)}

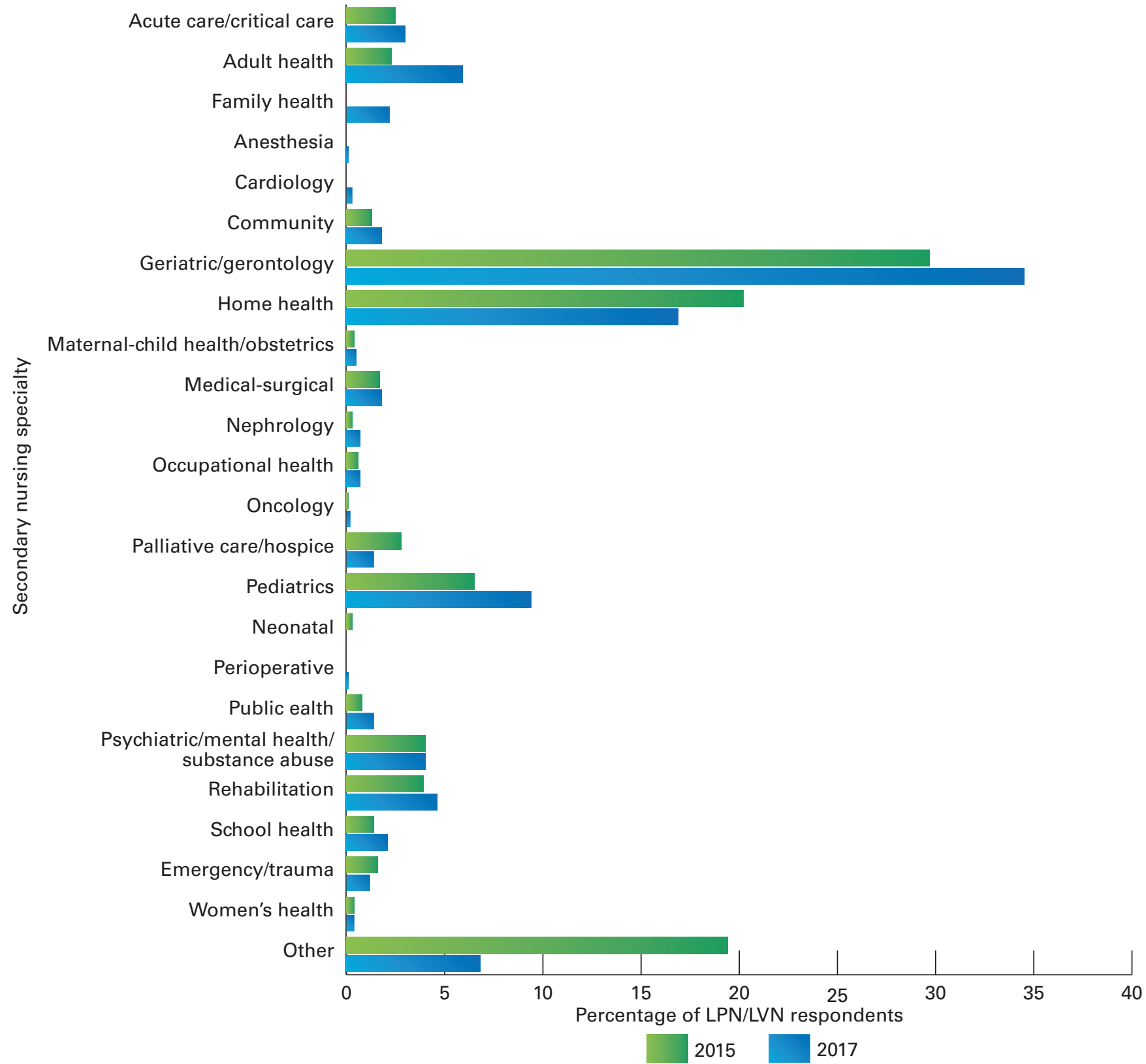

Note. Survey participants were asked to answer this question only if they were actively employed in nursing.

\section{Salary/Earnings}

2016 pre-tax annual earnings from primary nursing position

The median pre-tax annual earnings for responding LPNs/LVNs increased from $\$ 38,000$ in 2015 to $\$ 40,000$ in 2017. Categorically, the percentage of respondents earning less than $\$ 40,000$ annually decreased by 6.6 percentage points, while all other earnings categories increased. The percentage of respondents making between $\$ 40,000$ and $\$ 60,000$ increased by 3.6 percentage points; those making between $\$ 60,000$ and $\$ 80,000$ increased by 1.9 percentage points; and those making more than $\$ 80,000$ increased by 0.9 percentage points. See Table 65. 


\section{TABLE 65}

\section{Annual Earnings - LPN/LVN}

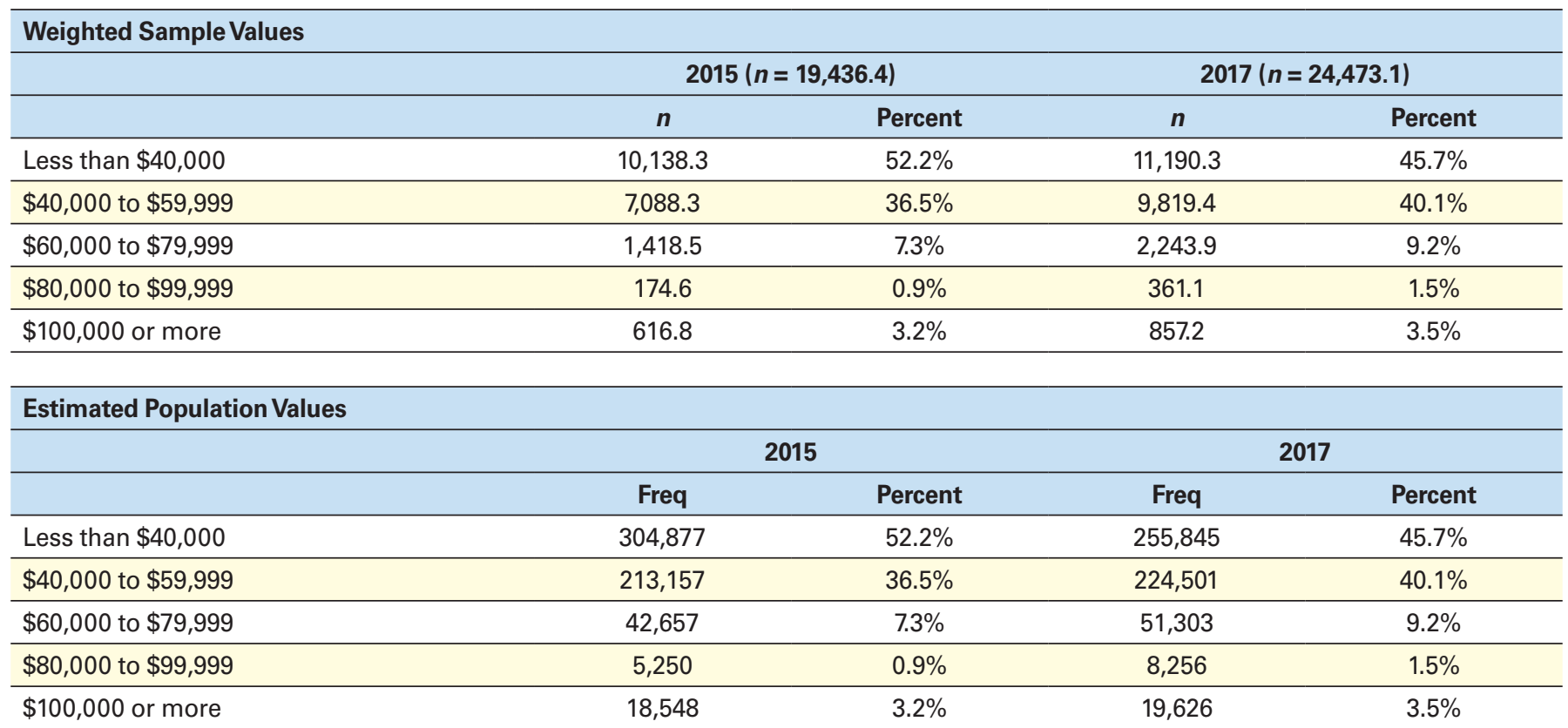

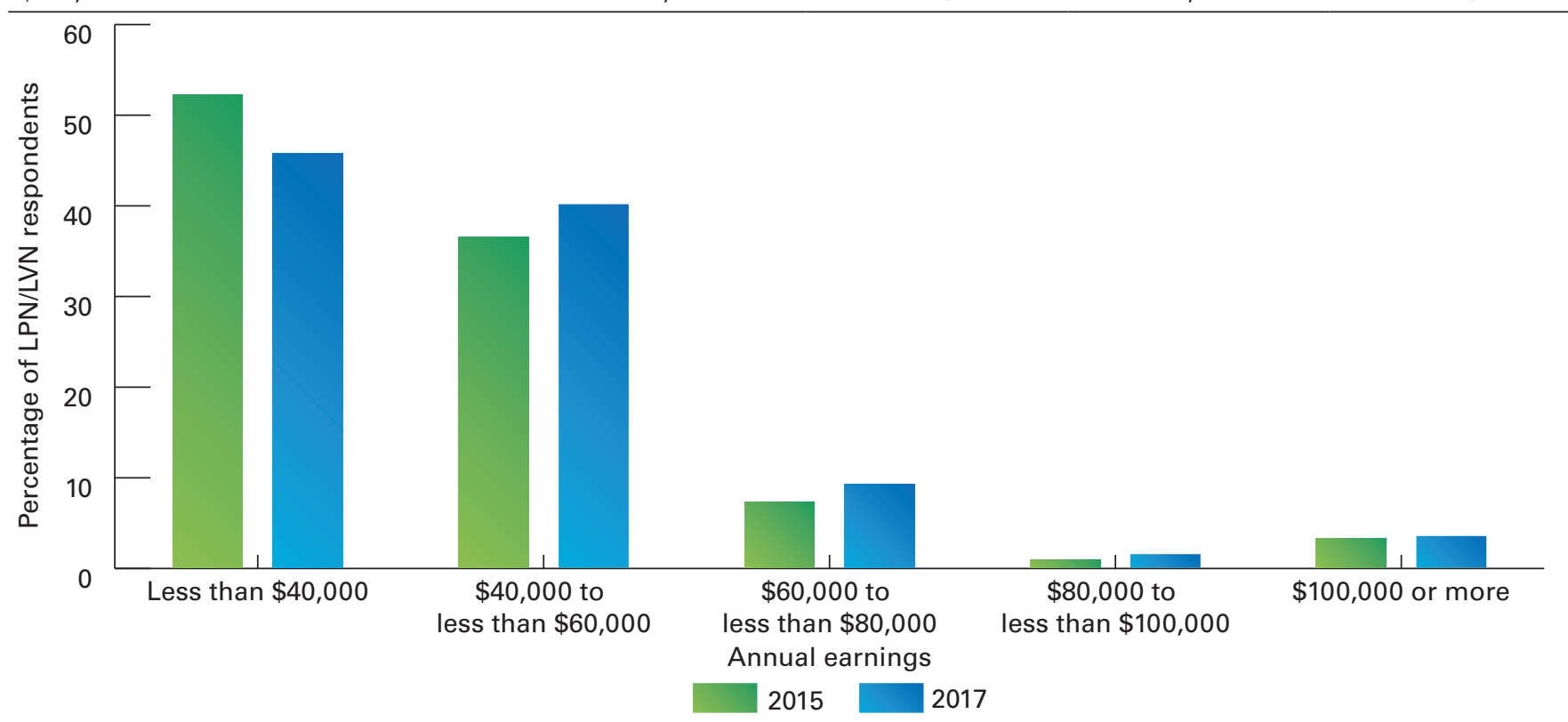

Note. Survey participants were asked to answer this question only if they were actively employed in nursing. Salary includes overtime and bonuses, but does not include sign-on bonuses.

\section{Earnings by gender and specialty}

For all specialties, the median salary for males is higher than the median salary for females. LPNs/LVNs working in rehabilitation have the highest median salary overall, and LPNs/LVNs working in school health have the lowest median salary overall. See Table 66. 


\section{TABLE 66}

\section{Median Annual Earnings by Gender and Specialty-LPN/LVN}

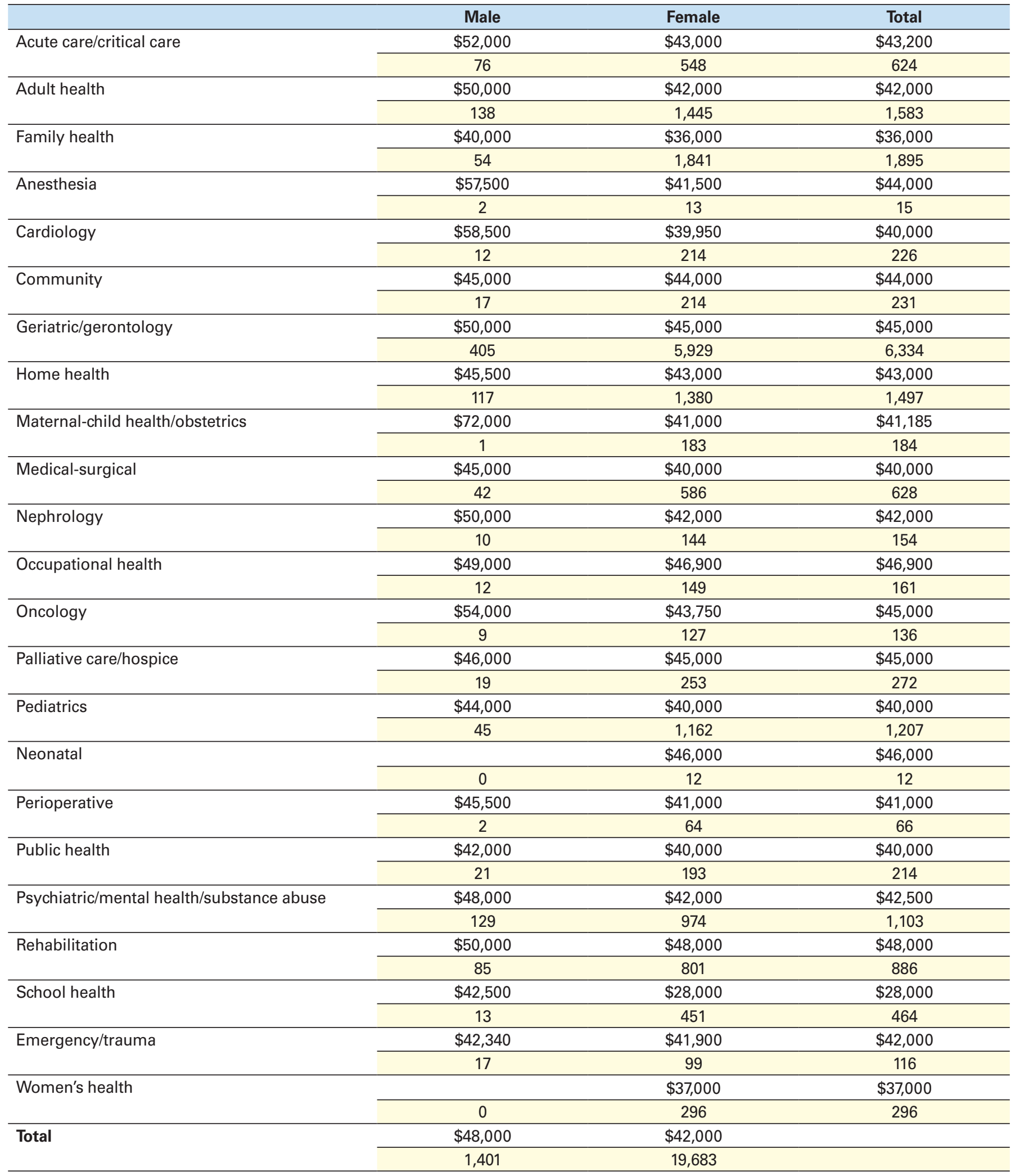

Note. Sample sizes are unweighted. Survey participants were asked to answer this question only if they were actively employed in nursing. 
LPNs/LVNs with baccalaureate and master's degrees have the highest median salaries. See Table 67

\section{TABLE 67}

\section{Median Annual Earnings by Highest Education - LPN/LVN}

\begin{tabular}{lcc}
\hline & Median Salary & $\boldsymbol{n}$ \\
\hline Vocational/practical certificate & $\$ 42,000$ & 16,750 \\
\hline Diploma & $\$ 41,600$ & 3,709 \\
\hline Associate degree & $\$ 40,000$ & 2,199 \\
\hline Baccalaureate degree & $\$ 51,619$ & 227 \\
\hline Note. Sample sizes are unweighted. Survey participants were asked to answer this question only if they were actively employed in nursing.
\end{tabular}

\section{Earnings by state}

Median earnings rose in most states. The highest median earnings were for LPNs/LVNs practicing in Alaska (\$52,000). The lowest median earnings were for LPNs/LVNs practicing in South Dakota (\$34,865), Alabama $(\$ 35,000)$, Mississippi (\$35,000), and West Virginia $(\$ 35,000)$. See Table 68 .

\section{TABLE 68}

\section{Median Annual Earnings in Primary Nursing Position by State(s) Where Currently Practicing-LPN/LVN}

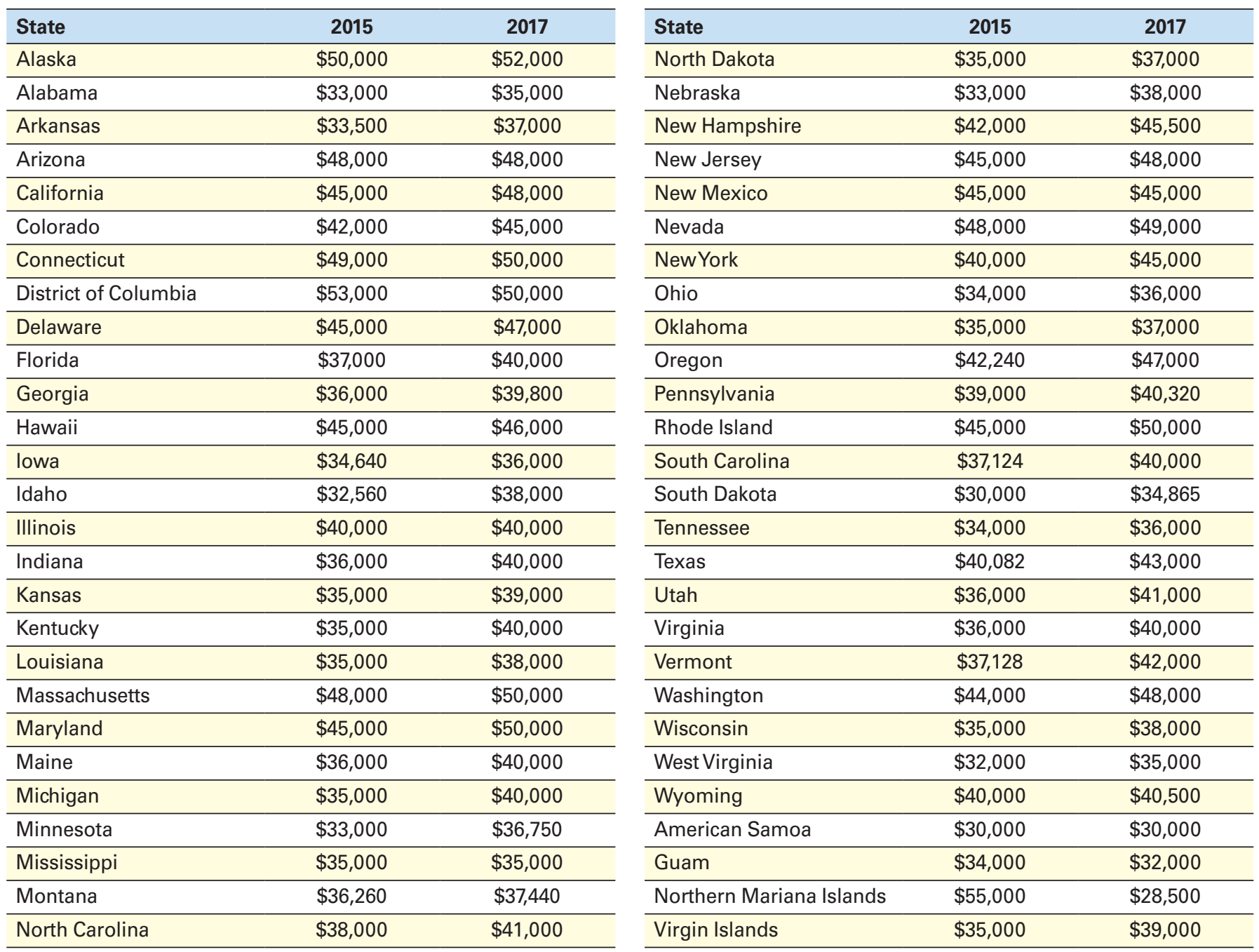

Note. Medians are unweighted. Survey participants were asked to answer this question only if they were actively employed in nursing. 
Earnings by years licensed and age

Similarly to RNs, there are steady increases in median salary as the number of years licensed increases, and little to no trend when LPNs/ LVNs increase in age. See Table 69

\section{TABLE 69}

\section{Median Annual Earnings by Years Licensed and Age-LPN/LVN}

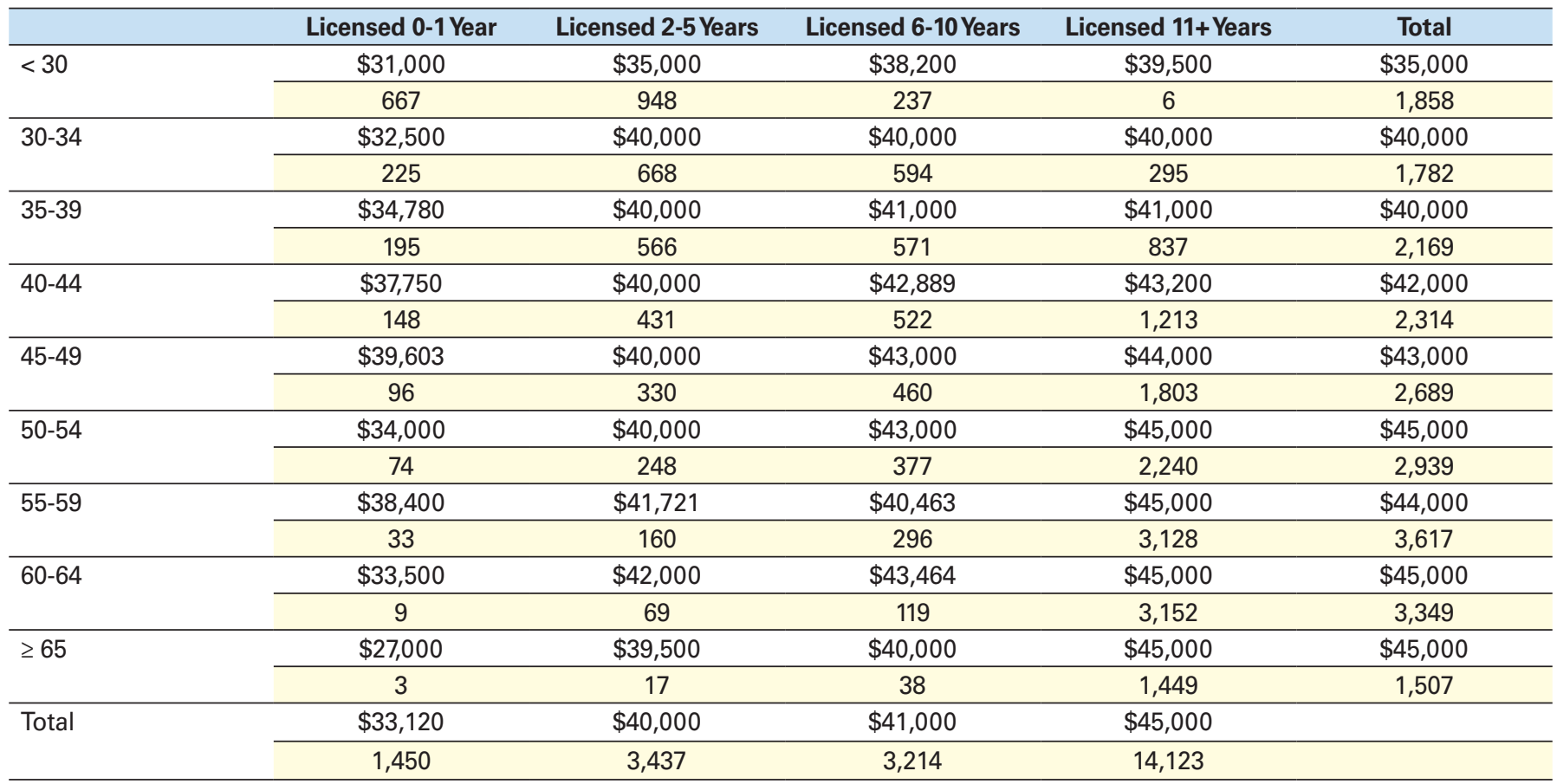

Note. Sample sizes are unweighted. Survey participants were asked to answer this question only if they were actively employed in nursing.

\section{Telehealth Utilization}

\section{Percent of time providing telehealth}

Responding LPNs/LVNs indicated that 54.2\% were providing nursing services remotely in 2017 compared to $46.1 \%$ in 2015 . Almost a quarter $(23.2 \%)$ of LPN/LVN respondents were spending up to $25 \%$ of their time providing services remotely. The largest increase occurred among those LPNs/LVNs who were providing nursing services remotely from 76 to $100 \%$ of the time, increasing from $7.2 \%$ in 2015 to $12.7 \%$ in 2017. See Table 70 .

\section{TABLE 70}

\section{Percent of Time Providing Telehealth - LPN/LVN}

\begin{tabular}{|c|c|c|c|c|}
\hline \multicolumn{5}{|c|}{ Weighted Sample Values } \\
\hline & \multicolumn{2}{|c|}{$2015(n=23,619.9)$} & \multicolumn{2}{|c|}{$2017(n=27,760.6)$} \\
\hline & $n$ & Percent & $n$ & Percent \\
\hline Never & $12,723.1$ & $53.9 \%$ & $12,715.8$ & $45.8 \%$ \\
\hline 1 to $25 \%$ & $5,496.6$ & $23.3 \%$ & $6,436.4$ & $23.2 \%$ \\
\hline 26 to $50 \%$ & $2,207.1$ & $9.3 \%$ & $2,821.3$ & $10.2 \%$ \\
\hline 51 to $75 \%$ & $1,490.6$ & $6.3 \%$ & $2,253.8$ & $8.1 \%$ \\
\hline
\end{tabular}




\begin{tabular}{|c|c|c|c|c|}
\hline \multicolumn{5}{|c|}{ Estimated Population Values } \\
\hline & \multicolumn{2}{|c|}{2015} & \multicolumn{2}{|c|}{2017} \\
\hline & Freq & Percent & Freq & Percent \\
\hline Never & 382,606 & $53.9 \%$ & 290,722 & $45.8 \%$ \\
\hline 1 to $25 \%$ & 165,294 & $23.3 \%$ & 147,157 & $23.2 \%$ \\
\hline 26 to $50 \%$ & 66,373 & $9.3 \%$ & 64,503 & $10.2 \%$ \\
\hline 51 to $75 \%$ & 44,826 & $6.3 \%$ & 51,529 & $8.1 \%$ \\
\hline
\end{tabular}

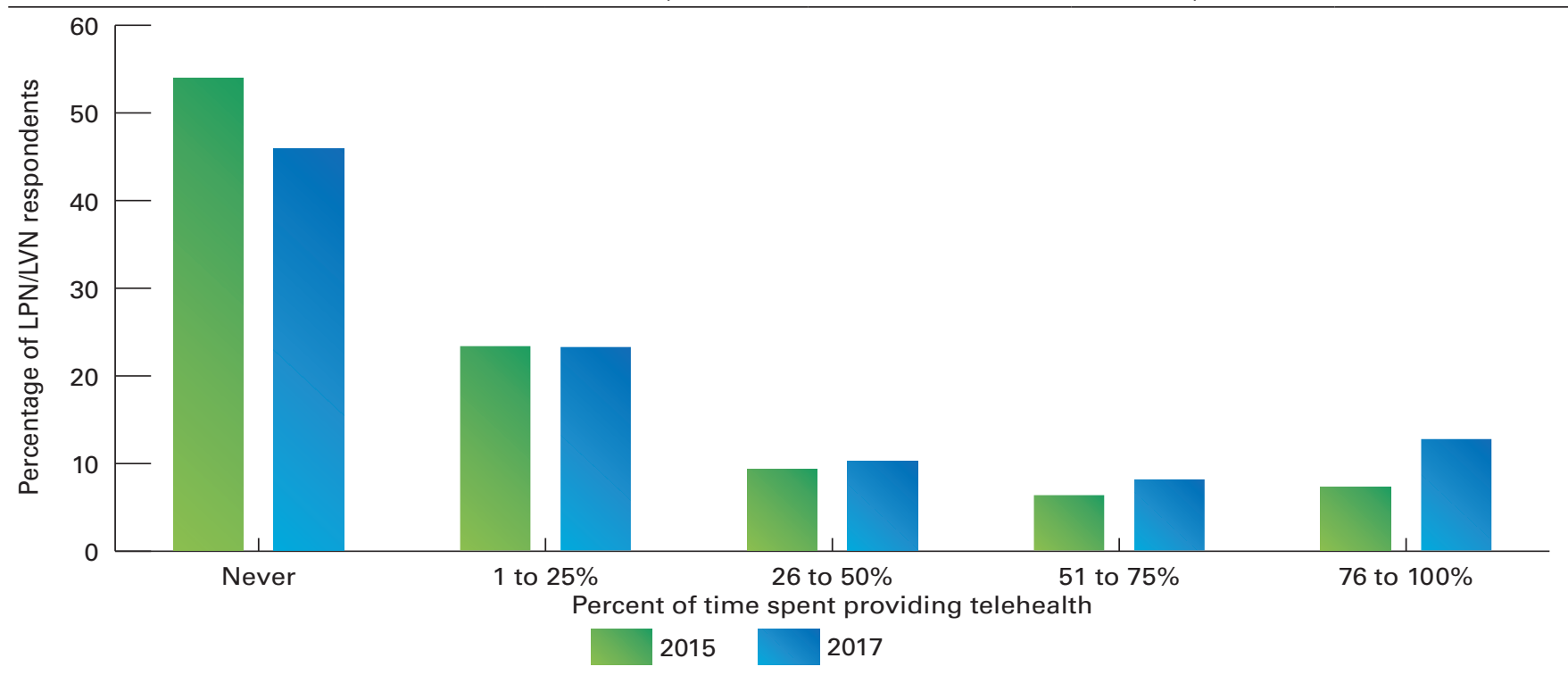

Note. Survey participants were asked to answer this question only if they were actively employed in nursing.

\section{Telehealth across state borders}

Of those providing nursing services remotely, $43.9 \%$, provided such services across state borders, compared to $33.8 \%$ in 2015 . About one-third of LPN respondents $(33.1 \%)$ provided such services to patients or clients across state borders up to $25 \%$ of the time. This represents a 5.6 percentage point increase from 2015. See Table 71.

\section{TABLE 71}

\section{Percent of Time Providing Telehealth Across State Borders-LPN/LVN}

\begin{tabular}{|c|c|c|c|c|}
\hline \multicolumn{5}{|c|}{ Weighted Sample Values } \\
\hline & \multicolumn{2}{|c|}{$2015(n=11,421.5)$} & \multicolumn{2}{|c|}{$2017(n=12,214.3)$} \\
\hline & $n$ & Percent & $n$ & Percent \\
\hline Never & $7,566.5$ & $66.2 \%$ & $6,849.9$ & $56.1 \%$ \\
\hline 1 to $25 \%$ & $3,140.4$ & $27.5 \%$ & $4,040.1$ & $33.1 \%$ \\
\hline 26 to $50 \%$ & 371.1 & $3.2 \%$ & 622.9 & $5.1 \%$ \\
\hline 51 to $75 \%$ & 180.4 & $1.6 \%$ & 372.2 & $3.0 \%$ \\
\hline
\end{tabular}




\section{Percent of Time Providing Telehealth Across State Borders - LPN/LVN (continued)}

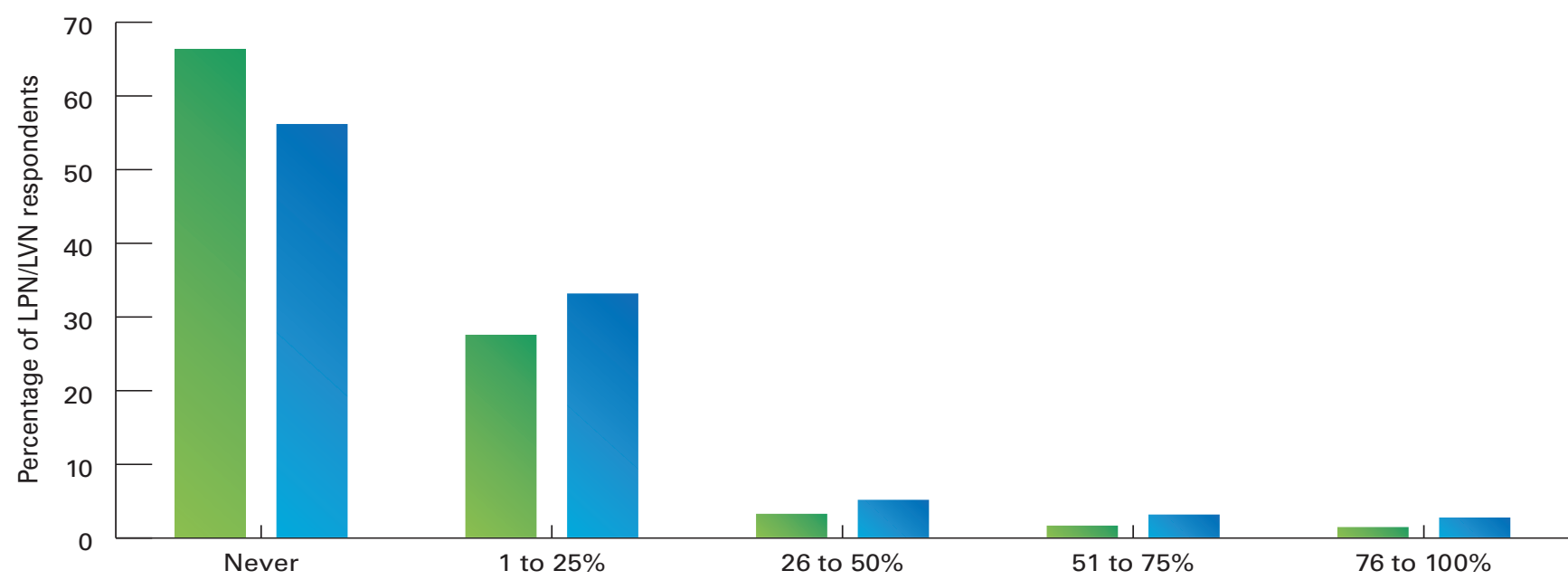

Percent of time spent providing telehealth across state borders

2015

2017

Note. Survey participants were asked to answer this question only if they were actively employed in nursing.

Telehealth across national borders

Only $11.8 \%$ of LPNs/LVNs providing remote services to patients or clients provide such services across national borders. In this survey, $7.3 \%$ of nurses reported doing so up to $25 \%$ of their time. See Table 72 .

\section{TABLE 72}

\section{Percent of Time Providing Telehealth Across National Borders-LPN/LVN}

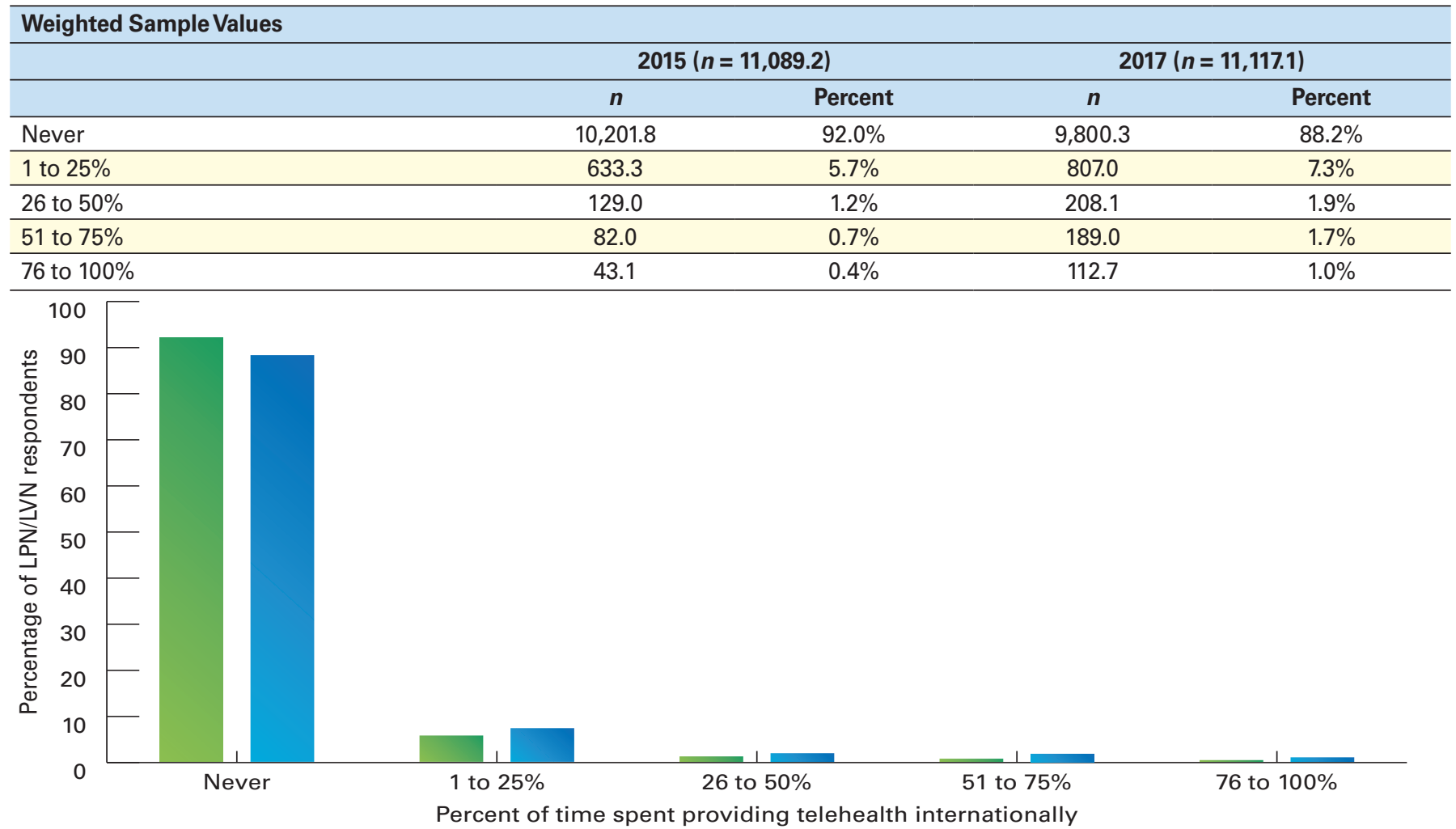

2015

2017

Note. Survey participants were asked to answer this question only if they were actively employed in nursing. 
Modes of communication used for telehealth

LPNs/LVNs providing services via phone or electronically to patients or clients were also asked to identify the modes of communication used. In this survey, 93.2\% of LPNs/LVNs reported using a telephone to communicate with patients or clients, a 2.5 percentage point decrease from 2015; 28.9\% reported using email to communicate with patients or clients, which is a decrease from the $29.5 \%$ who indicated so in 2015; and 22.5\% reported using electronic messaging for patient/client communication in 2017 compared to $21.9 \%$ in 2015 . See Table 73.

\section{TABLE 73}

\section{Modes of Communication Used for Telehealth - LPN/LVN}

\begin{tabular}{|c|c|c|c|c|}
\hline \multicolumn{5}{|c|}{ Weighted Sample Values } \\
\hline & \multicolumn{2}{|c|}{$2015(n=8,881.7)$} & \multicolumn{2}{|c|}{$2017(n=11,164.0)$} \\
\hline & $n$ & Percent & $n$ & Percent \\
\hline VolP & 187.7 & $2.1 \%$ & 349.6 & $3.1 \%$ \\
\hline Virtual ICU & 84.9 & $1.0 \%$ & 125.2 & $1.1 \%$ \\
\hline Email & $2,622.2$ & $29.5 \%$ & $3,226.2$ & $28.9 \%$ \\
\hline Video call & 207.4 & $2.3 \%$ & 260.7 & $2.3 \%$ \\
\hline Other & 883.0 & $9.9 \%$ & $1,027.2$ & $9.2 \%$ \\
\hline
\end{tabular}

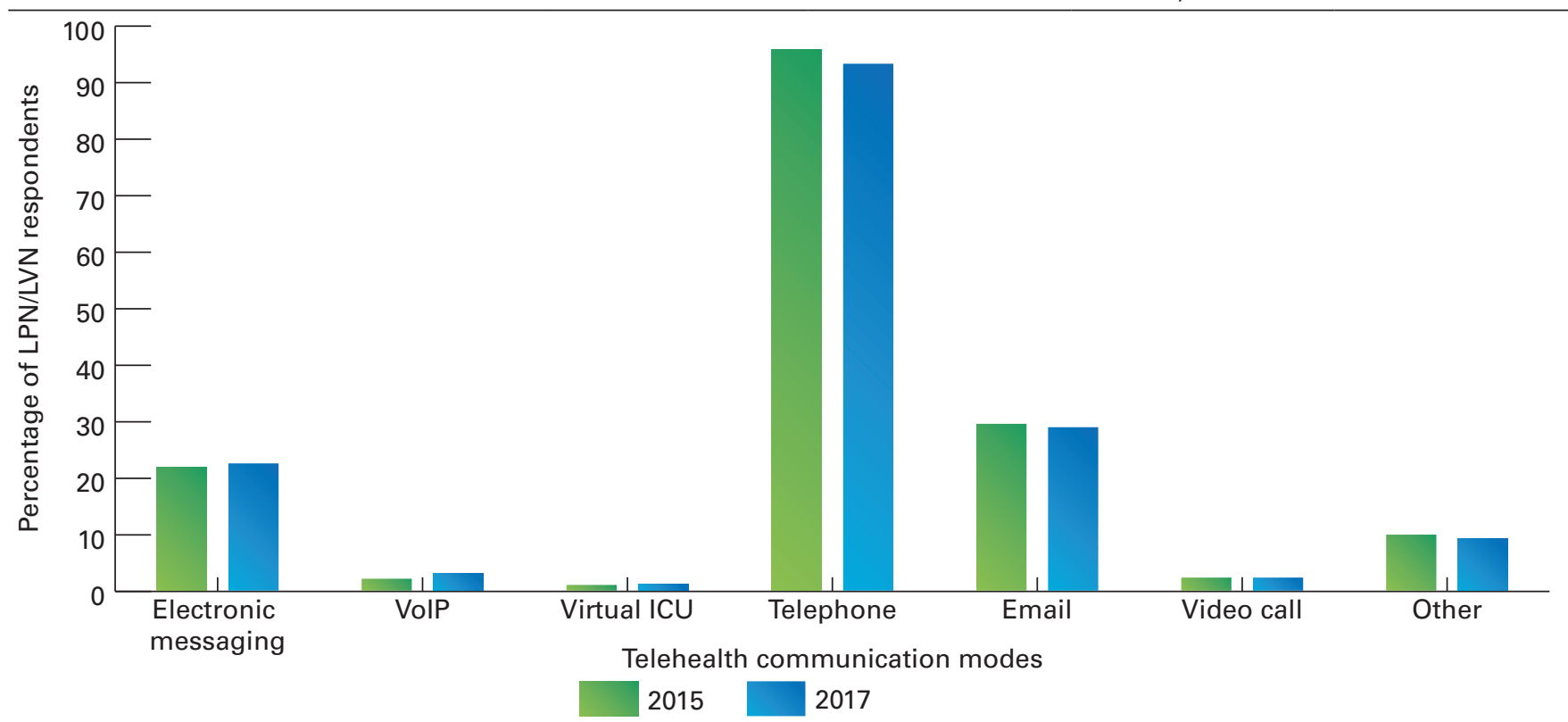

Note. Survey participants were asked to answer this question only if they were actively employed in nursing. Respondents were asked to select all that apply. VoIP = voice over Internet protocol; ICU = intensive care unit.

\section{Discussion and Implications}

Approximately 152,000 LPNs/LVNs were included in the sample; 40,272 responded (26.5\%). These data provide the most recent and detailed information on characteristics of licensed LPNs/LVNs, such as age, diversity, education, employment setting, position title, employment specialty, and salary. Data and research on the nursing workforce are often focused more on RNs and less so on LPNs/LVNs; therefore, the present study was a critical addition to nursing workforce data.

In terms of diversity, $29 \%$ of responding LPNs/LVNs identified themselves as a minority, with the largest reporting minority group being Black/African American, at 18.5\%. Of the respondents, $71 \%$ were White/Caucasian. This study's racial/ethnic diversity results are somewhat dissimilar to HRSA's (2017) nursing projections report by racial/ethnic diversity of the U.S. LPN/LVN workforce for 2014, which found a $61 \%$ White (non-Hispanic) LPN/LVN workforce and a 24\% Black LPN/LVN workforce. Coffman, Bates, Chan, and Spetz (2015) reported a 61\% White workforce, and the largest reporting minority group was Black/African American, at 24\%. Concerning 
gender diversity, $7.7 \%$ of the LPN/LVN respondents were male, which is somewhat similar to two other recent analyses of gender in the LPN/LVN workforce at 9\% (Coffman, Bates, Chan, \&, Spetz, 2015) and 7.6\% (HRSA, 2017).

While $83 \%$ of respondents indicated a vocational/practical certificate was their entry qualification for their first U.S. nursing license, three-quarters (77.8\%) of LPNs/LVNs did not receive higher degrees. This represents a slight increase of 1.8 percentage points from 2015 (76.0\%). The vast majority (97.5\%) were educated in the United States, which is consistent with results from the 2015 survey (98.5\%). This is similar to Coffman and colleagues' (2015) finding that $76 \%$ did not hold a college degree in preparation to be an LPN/LVN. Of respondents, $14.3 \%$ indicated they held a diploma in nursing and another $7.3 \%$ held an associate degree in nursing as their highest level of nursing education. Almost a quarter of responding LPNs/LVNs indicated they had a non-nursing degree. Of those respondents, $68.8 \%$ reported an associate level non-nursing degree, $25.0 \%$ reported a baccalaureate level non-nursing degree, and an additional $6.2 \%$ reported a graduate-level non-nursing degree.

The most common care setting of LPN/LVN respondents was in nursing home/extended care ( $31.7 \%$ ), followed by $14 \%$ in home health and $9.6 \%$ in hospitals. These percentages are similar to those found in the 2015 survey. Approximately $30.5 \%$ of LPNs/LVNs reported their primary practice specialty as geriatric/gerontology compared with $27.7 \%$ in the 2015 survey. These findings support a report by Coffman et al. (2015) that indicated long-term care employs more LPNs/LVNs than any other industry. In fact, they found the number of LPNs/LVNs working in long-term care increased from 258,670 in 2008 to 289,946 in 2013, an increase of 13\%, while the number of LPNs/LVNs working in hospitals, outpatient care, and other sectors decreased by 20\%. Over two-thirds (73.1\%) of LPNs/ LVNs' primary title was staff nurse. The current survey also found that $54.2 \%$ of LPNs/LVNs provided patient care services utilizing telehealth technologies, representing an increase of 8 percentage points. Of those, $43.9 \%$ were providing electronic services across state borders, compared to $33.8 \%$ in 2015 .

\section{Limitations}

Limitations of the current study include a response rate of $26.5 \%$, which was lower than the response rate for the similar sample of RNs (32.8\%). A nonresponse bias analysis was conducted and indicated a slight overrepresentation of three distinct variables: White/Caucasian, female, and age 50 and older in the responding sample. As described earlier, gender and age were used to make nonresponse weighting adjustments, along with weighting adjustments for differing nursing population sizes across states. An additional limitation was missing or incomplete data. While the large sample size partially compensated for this, certain item nonresponse bias could have impacted the results, such as salary (30.0\% did not answer): Both upper and lower limits had been set on the salary figures; however, extreme values could still have skewed mean values upward; the medians were likely the more accurate statistic versus the mean for the measure of central tendency. Data used for comparison may have quite different samples; for example, the HRSA (2017) brief on gender and racial/ethnic diversity of U.S. health occupations used data on a U.S. working-age population age 16 or older who were currently employed or who were recently employed and were seeking employment (e.g., individuals who were recently laid off), and the Coffman et al. (2015) report relied on data from the American Community Survey and the Public Use Microdata Sample. Lastly, questions may be altered slightly from survey to survey, making comparisons potentially more challenging.

\section{Conclusion}

The current LPN/LVN workforce is expected to change in terms of the ratios of racial/ethnic minority groups and of men to women, though those changes are gradual. The LPN/LVN workforce is still largely composed of White/Caucasian women. Changes in the health care environment are especially relevant for LPNs/LVNs because where they are working is changing as more and more health care is delivered outside acute-care settings. Recent trends indicate strong demand for LPNs/LVNs in long-term care and community settings. The most recent HRSA projections on the supply and demand for LPNs/LVNs indicate there will be a shortage of 151,000 nurses by 2030 , affecting all but 18 states (HRSA 2017). Of concern are the projections by Coffman et al. (2015) that indicate the LPN/LVN workforce in the United States is declining in size, from 675,918 in 2008 to 635,975 in 2013, a decrease of 6\%. This decline is happening at a time when the supply and demand of LPNs/LVNs are likely to be affected by such factors as population growth, aging of the population, preferences for care delivery, and economic conditions. 


\section{References}

American Association of Colleges of Nursing. (2017a). DNP fact sheet. Retrieved from www.aacnnursing.org/News-Information/Fact-Sheets/ DNP-Fact-Sheet

American Association of Colleges of Nursing. (2017b). Degree completion programs for registered nurses: $R N$ to masters degree and $R N$ to baccalaureate programs. Retrieved from www.aacnnursing.org/News-Information/ Fact-Sheets/Degree-Completion-Programs

American Association of Colleges of Nursing. (2017c). Nursing faculty shortages. Retrieved from http://www.aacnnursing.org/News-Information/ Fact-Sheets/Nursing-Faculty-Shortage

American Association for Public Opinion Research. (n.d.). Response ratesAn overview. Retrieved from www.aapor.org/Education-Resources/ For-Researchers/Poll-Survey-FAQ/Response-Rates-An-Overview. aspx

American Well. American Well ${ }^{\circledR} 2015$ Telehealth Survey: 64\% of Consumers Would See a Doctor Via Video. Retrieved from https://www. americanwell.com/press-release/american-well-2015-telehealth-survey-64-of-consumers-would-see-a-doctor-via-video/

Budden, J. S., Moulton, P., Harper, K. J., Brunell, M. L., \& Smiley, R. (2016). The 2015 national nursing workforce survey. Journal of Nursing Regulation, 7(1), S1-S90.

Buerhaus, P. I., Skinner, L. E., Auerbach, D. I., \& Staiger, D. O. (2017). State of the registered nurse workforce as a new era of health emerges. Nursing Economics, 35(5), 229-237.

Campaign Progress. (2017). Welcome to the future of nursing: Campaign for Action dashboard. Retrieved from https://campaignforaction.org/wpcontent/uploads/2017/07/Dashboard-Indicators_Dec17.pdf

Coffman, J. Bates, T., Chan, K., \& Spetz, J. (2015). Trends in the licensed practical nurse workforce from 2008 to 2013. The Gerontologist, 55(2), 776.

Health Resources and Services Administration. (2017, December). Nursing workforce projections by ethnicity and race: 2014-2030. Retrieved from https://bhw.hrsa.gov/sites/default/files/bhw/health-workforceanalysis/research/projections/hrsa-bhw-rn-lpn-factsheet-12-17.pdf
HIMSS Analytics. (2015). 2015 telemedicine study. https://www.himssanalytics.org/research/essentials-brief-telemedicine-study

Leiber, N. (2016). Europe bets on robots to help care for seniors. Retrieved from www.bloomberg.com/news/articles/2016-03-17/europe-bets-on-robots-tobelp-care-for-seniors

Masui, A. (2016). Development of care robots growing in aging Japan. The Japanese Times. Retrieved from www.japantimes.co.jp/news/2016/01/27/ national/social-issues/development-care-robots-growing-agingjapan/\#.W4KyOuhKhEY

National Academy of Medicine. (2010). The future of nursing: Leading change, advancing health.

National Academy of Medicine. (2015). Assessing progress on the IOM Report The Future of Nursing. Retrieved from www.nationalacademies.org/ $\mathrm{hmd} /$ Reports/2015/Assessing-Progress-on-the-IOM-Report-TheFuture-of-Nursing.aspx.

National Council of State Boards of Nursing. (2018). Enhanced Nurse Licensure Compact (eNLC) implementation. Retrieved from www.ncsbn. org/enhanced-nlc-implementation.htm

Susskind, R., \& Susskind, D. (2015). The future of the professions: How technology will transform the work of human experts. Cary, NC: Oxford University Press;.

U.S. Bureau of Labor Statistics. (2017). Occupational employment statistics, May 2016. Retrieved from www.bls.gov/oes/current/oes291141. btm

U.S. Bureau of Labor Statistics. (2018). Occupational outlook handbook. Retrieved from www.bls.gov/ooh/health care/registered-nurses.htm

U.S. Census Bureau. (2017a). National population totals tables: 2010-2016. Retrieved from www.census. gov/data/tables/2016/demo/popest/nationtotal.html

U.S. Census Bureau. (2017b). Quick facts. Retrieved from www.census. gov/quickfacts/fact/table/US/PST045217 


\section{Appendices}

\section{APPENDIX A}

\section{Registered Nurse Nonresponse Analysis and Sample Weighting}

A formal nonresponse bias analysis was conducted following the close of the survey. Although response rates are a valuable indicator of survey quality, they are not a good measure of response bias. An analysis of basic demographic data (i.e., gender, age, race/ethnicity, number of years since graduation, number of years since first licensed) for all registered nurse (RN) licensees sampled from the Nursys ${ }^{\circledR}$ database was used to compare the survey respondents and nonrespondents to determine the representativeness of the survey participants. Variables in the data file came from both the Nursys database (i.e., the frame data) and responses to the survey (i.e., survey data). The variables used in the nonresponse analysis were from the frame and include state, date of birth, gender, and ethnicity. The dependent variable in the analysis was whether or not the sampled $\mathrm{RN}$ completed the questionnaire.

\section{Descriptive statistics}

Of the 153,920 RNs in the sample frame, 48,704 responded, for a response rate of $31.6 \%$ (Table A1). Tables A2 and A3 show the frequencies for the categorical variables of gender and race/ethnicity. Table A4 shows the descriptive statistics for age. The only demographic information used for the following analyses come from Nursys, and are not the demographic data received from the survey.

\section{TABLE A1}

\section{Response Rate-RN}

\begin{tabular}{lcc}
\hline & $(\boldsymbol{n}=\mathbf{1 5 3 , 9 2 0 )}$ & Percent \\
\hline Nonresponse & 105,216 & $68.4 \%$ \\
\hline Response & 48,704 & $31.6 \%$ \\
\hline
\end{tabular}

\section{TABLE A2}

\section{Gender of Sample-RN}

\begin{tabular}{lcc}
\hline & $(\boldsymbol{n}=\mathbf{1 5 3 , 9 2 0})$ & Percent \\
\hline Female & 95,567 & $62.1 \%$ \\
\hline Male & 9,985 & $6.5 \%$ \\
\hline Restricted/unknown & 40,248 & $26.1 \%$ \\
\hline Missing & 8,120 & $5.3 \%$
\end{tabular}

This response rate corresponds to the American Association for Public Opinion Research Response Rate 1 (the minimum response rate), in which the numerator is the number of completed questionnaires and the denominator is the total sample size (American Association for Public Opinion Research, n.d.).

\section{TABLE A3}

\section{Race/Ethnicity of Sample-RN}

\begin{tabular}{lcc}
\hline & $(\boldsymbol{n}=\mathbf{1 5 3 , 9 2 0 )}$ & Percent \\
\hline White/Caucasian & 35,518 & $23.1 \%$ \\
\hline Black/African American & 2,697 & $1.8 \%$ \\
\hline Asian & 1,499 & $1.0 \%$ \\
\hline Hispanic & 1,957 & $1.3 \%$ \\
\hline Native American & 356 & $0.2 \%$ \\
\hline Pacific Islander & 47 & $<0.1 \%$ \\
\hline Other & 905 & $0.6 \%$ \\
\hline Restricted/unknown & 40,248 & $26.1 \%$ \\
\hline Missing & 70,693 & $45.9 \%$ \\
\hline
\end{tabular}

TABLE A4

\section{Age of Sample-RN}

\begin{tabular}{cccccc}
\hline & $\boldsymbol{n}$ & Mean & $\begin{array}{c}\text { Standard } \\
\text { Deviation }\end{array}$ & Minimum & Maximum \\
\hline Age & 106,402 & 46.8 & 13.6 & 16 & 109 \\
\hline
\end{tabular}

\section{Bivariate Analysis}

Tables A5 and A6 show the bivariate relationships between the demographic variables from the sample frame and whether or not the respondent completed the survey. Table A5 shows there were far fewer men in the database (9,985 compared to 95,567 women) and they were less likely to complete the survey (25.2\% compared to $34.1 \%$ women).

\section{TABLE A5}

\section{Response Rate by Gender-RN}

\begin{tabular}{lccc}
\hline \multicolumn{3}{c}{ Completed Survey } \\
\hline Female & $\boldsymbol{n}$ & No & Yes \\
\hline Male & 95,567 & $65.9 \%$ & $34.1 \%$ \\
\hline Total & 9,985 & $74.8 \%$ & $25.2 \%$ \\
\hline Note. $\chi 2(1, N=105,552)=319.5, p<.001$. & $33.2 \%$ \\
\hline
\end{tabular}




\section{TABLE A6}

\section{Response Rate by Race/Ethnicity-RN}

\begin{tabular}{lccc}
\hline & \multicolumn{3}{c}{ Completed Survey } \\
\hline Race/Ethnicity & $\boldsymbol{n}$ & No & Yes \\
\hline White/Caucasian & 35,518 & $66.3 \%$ & $33.7 \%$ \\
\hline African American & 2,697 & $77.7 \%$ & $22.3 \%$ \\
\hline Hispanic & 1,957 & $75.1 \%$ & $24.9 \%$ \\
\hline Asian & 1,499 & $73.4 \%$ & $26.6 \%$ \\
\hline Native American & 356 & $77.5 \%$ & $22.5 \%$ \\
\hline Pacific Islander & 47 & $85.1 \%$ & $14.9 \%$ \\
\hline Other & 905 & $74.3 \%$ & $25.7 \%$ \\
\hline Total & 42,979 & $67.9 \%$ & $32.1 \%$ \\
\hline
\end{tabular}

Note. c2 $(6, N=42,979)=266.0 p<.001$.

Table A7 displays the mean age of RNs of those who did and did not respond. On average, those who completed the survey were 5.4 years older than the nonrespondents. This relationship is statistically significant.

TABLE A7

\section{Age by Response and Nonresponse-RN}

\begin{tabular}{|c|c|c|}
\hline Completed Survey & & Age \\
\hline \multirow[t]{3}{*}{ No } & $n$ & 70,748 \\
\hline & Mean & 44.9 \\
\hline & Standard deviation & 13 \\
\hline \multirow[t]{3}{*}{ Yes } & $n$ & 35,654 \\
\hline & Mean & 50.4 \\
\hline & Standard deviation & 14.1 \\
\hline \multirow[t]{3}{*}{ Total } & $n$ & 106,402 \\
\hline & Mean & 46.8 \\
\hline & Standard deviation & 13.6 \\
\hline
\end{tabular}

Note. A t-test shows that the average age among those who did and did not respond is significantly different at the $<.001$ level.

\section{Weights}

In The 2015 National Nursing Workforce Survey, nonresponse adjustments were made for gender and age. For the 2017 survey, nonresponse adjustments were applied for gender and age in the jurisdictions for which data were obtained through the Nursys database. There was a high degree of missing race/ethnicity data in the sample frame (72.1\% missing/unknown). Therefore, it was deemed impractical to use race/ethnicity for nonresponse adjustment even though there were differences in response rates by the category.

In order to create the combined age and gender (Age $\times$ Gender) nonresponse weights, the survey response rates for the age variable were compared at the 5-year age-group level and neighboring cells with similar response rates were collapsed. Upon completion of this process, six age-groups were created (16 to 49,50 to 54,55 to 59 ,
60 to 64,65 or older, missing). These six age-groups were combined with the gender variable response categories (male, female, missing) to produce 18 Age $\times$ Gender categories. The survey response rate for each Age $\times$ Gender category (\# responding/\# in sample frame) was calculated and used to create each category's weight as follows:

$$
\begin{gathered}
\text { Age } \times \text { Gender Category Weight }=\frac{\text { Overall Survey Response Rate }}{\text { Age } \times \text { Gender Category Survey }} \\
\text { Response Rate }
\end{gathered}
$$

As an example of how this was calculated, there were 434 RNs in the sample frame whose gender was identified as male and whose age was missing. Out of these $434 \mathrm{RNs}, 87$ responded. The Age $\times$ Gender response rate for this category was determined to be $87 / 434=.2005$. The overall survey response rate in jurisdictions for which data was obtained through the Nursys database was $37,548 / 113,672=.3303$. So the Age $\times$ Gender weight for the age missing-gender male category was $.3303 / .2005=1.648$.

Respondents in jurisdictions for which data were not obtained through the Nursys database were not weighted for age and gender and therefore all assigned an Age $\times$ Gender weight of 1.000. When the Age $\times$ Gender weights for each respondent are totaled, the sum comes to 48,704 , the same as the total number of respondents. Table A8 displays the weights for the 18 Age $\times$ Gender categories in jurisdictions for which data were obtained through the Nursys database.

\section{TABLE A8}

\section{Age $\times$ Gender Response Rate-RN}

\begin{tabular}{lccc}
\hline & & Gender \\
\hline Age-Group & Invalid & Female & Male \\
\hline $16-49$ & 1.387 & 1.225 & 1.610 \\
\hline $50-54$ & 1.053 & 0.974 & 1.210 \\
\hline $55-59$ & 0.872 & 0.822 & 1.033 \\
\hline $60-64$ & 0.791 & 0.709 & 0.904 \\
\hline 65 or older & 0.641 & 0.605 & 0.720 \\
\hline Age missing & 1.120 & 1.315 & 1.648
\end{tabular}

In a similar manner, poststratification weights were constructed at the state level to adjust for differing sampling rates across states. These adjustments were made by comparing the number of responses to the number of licensees in that state. Analysis of the raw data, without accounting for the sample design, would lead to the overall results being too heavily influenced by states with fewer licensees.

For example, there were 427,892 RNs in California, out of which 1,020 responded. The California re-sponse per license rate was $1,020 / 427,892=.0024$. The overall response per license rate was $48,704 / 4,639,548=.0105$. So the poststratification weight for California was $.0105 / .0024=4.375$.

Overall weights combined the Age $\times$ Gender and poststratification weights and were created by multiplying the Age $\times$ Gender and poststratification weights for each individual to create an initial 
set of weights, adding the initial weights together, and slightly adjusting the weights so that they sum up to 48,704 .

The overall weights adjust the distribution across states, age, and gender, but sum to the actual number of RNs in the subset of completed responses. They can be applied when analyzing relationships between variables without the effect of artificially altering the degrees of freedom and thereby affecting significance tests. The Age $\times$ Gender weights, poststratification weights, and overall weights are summarized in Table A9.

\section{TABLE A9}

\section{Weight Summary Statistics-RN}

\begin{tabular}{lccccc}
\hline & $\boldsymbol{n}$ & Minimum & Maximum & Sum & Mean \\
\hline Age by gender & 48,704 & 0.605 & 1.648 & 48,704 & 1.000 \\
\hline Poststratification & 48,704 & 0.088 & 4.792 & 48,704 & 1.000 \\
\hline Combined & 48,704 & 0.053 & 5.932 & 48,704 & 1.000 \\
\hline
\end{tabular}

Note. Combined weight was used in reporting results. 
APPENDIX B

\section{Licensed Practical Nurse/LicensedVocational Nurse Nonresponse Analysis and Sample Weighting}

As with the RNs, a formal nonresponse bias analysis was conducted of the LPN/LVN data following the close of the survey. Variables in the data file came from both the Nursys ${ }^{\circledR}$ database (i.e., the frame data) and responses to the survey (i.e., survey data). The variables used in the nonresponse analysis were from the frame and include state, date of birth, gender, and race/ethnicity. The dependent variable in the analysis was whether or not the sampled LPN/LVN completed the questionnaire.

\section{Preliminary analysis}

Of the 158,502 LPNs/LVNs in the sample frame, 40,272 responded, for a response rate of $25.4 \%$ (Table B1). Tables B2 and B3 show the frequencies for the categorical variables. Table B4 shows the descriptive statistics for age.

\section{TABLE B1}

\section{Response Rate-LPN/LVN}

\begin{tabular}{lcc}
\hline & $(\boldsymbol{n}=\mathbf{1 5 8 , 5 0 2})$ & Percent \\
\hline No & 118,230 & $74.6 \%$ \\
\hline Yes & 40,272 & $25.4 \%$ \\
\hline
\end{tabular}

TABLE B2

\section{Gender of Sample-LPN/LVN}

\begin{tabular}{lcc}
\hline & $(\boldsymbol{n}=\mathbf{1 5 8 , 5 0 2 )}$ & Percent \\
\hline Female & 92,886 & $58.6 \%$ \\
\hline Male & 8,550 & $5.4 \%$ \\
\hline Restricted/unknown & 46,215 & $29.2 \%$ \\
\hline Missing & 10,851 & $6.8 \%$ \\
\hline
\end{tabular}

\section{TABLE B3}

\section{Race/Ethnicity of Sample-LPN/LVN}

\begin{tabular}{lcc}
\hline & $(\boldsymbol{n}=\mathbf{1 5 8 , 5 0 2 )}$ & Percent \\
\hline White/Caucasian & 33,233 & $21.0 \%$ \\
\hline Black/African American & 6,188 & $3.9 \%$ \\
\hline Asian & 724 & $0.5 \%$ \\
\hline Hispanic & 2,823 & $1.8 \%$ \\
\hline Native American & 523 & $0.3 \%$ \\
\hline Pacific Islander & 71 & $<0.1 \%$ \\
\hline Other & 847 & $0.5 \%$ \\
\hline Restricted/unknown & 46,215 & $29.2 \%$ \\
\hline Missing & 67,878 & $42.8 \%$ \\
\hline
\end{tabular}

Volume 9/Issue 3 Supplement October 2018 (updated January 2019)

\section{TABLE B4}

Age of Sample-LPN/LVN

\begin{tabular}{cccccc}
\hline & $\mathbf{n}$ & Mean & $\begin{array}{c}\text { Standard } \\
\text { Deviation }\end{array}$ & Minimum & Maximum \\
\hline Age & 102,825 & 47.3 & 13.2 & 16 & 96 \\
\hline
\end{tabular}

\section{Bivariate analysis}

Tables B5 and B6 show the bivariate relationships between the demographic variables from the sample frame and whether or not the respondent completed the survey. There were far fewer men in the database (8,550 compared to 92,886 women) and they were less likely to complete the survey ( $19.6 \%$ compared to $27.9 \%$ women).

\section{TABLE B5}

\section{Response Rate by Gender-LPN/LVN}

\begin{tabular}{lccc}
\hline \multicolumn{3}{c}{ Completed Survey } \\
\hline Female & $\boldsymbol{n}$ & No & Yes \\
\hline Male & 82,886 & $72.1 \%$ & $27.9 \%$ \\
\hline Total & 101,436 & $80.4 \%$ & $19.6 \%$ \\
\hline Note. c2 $(1, N=101,436)=275.6, p<.001$. & $72.8 \%$ & $27.2 \%$ \\
\hline
\end{tabular}

From Table B6, nurses who identified as White/Caucasian were most likely to respond, with a response rate of $29.0 \%$. Those who responded other and Pacific Islander nurses were least likely to respond, with response rates of $19.0 \%$ and $18.3 \%$.

\section{TABLE B6}

\section{Response Rate by Race/Ethnicity-LPN/LVN}

\begin{tabular}{lccc}
\hline & & \multicolumn{2}{c}{ Completed Survey } \\
\hline Race/Ethnicity & $\boldsymbol{n}$ & No & Yes \\
\hline White/Caucasian & 33,233 & $71.0 \%$ & $29.0 \%$ \\
\hline African American & 6,188 & $79.8 \%$ & $20.2 \%$ \\
\hline Asian & 724 & $75.8 \%$ & $24.2 \%$ \\
\hline Hispanic & 2,823 & $78.1 \%$ & $21.9 \%$ \\
\hline Native American & 523 & $75.5 \%$ & $24.5 \%$ \\
\hline Pacific Islander & 71 & $81.7 \%$ & $18.3 \%$ \\
\hline Other & 847 & $81.0 \%$ & $19.0 \%$ \\
\hline Total & 44,409 & $73.0 \%$ & $27.0 \%$
\end{tabular}

Note. c2 $(6, N=44,409)=287.1, p<.001$.

Table B7 displays the mean age of LPNs/LVNs by response or nonresponse. On average, those who completed the survey were 
5.6 years older than the nonrespondents. This relationship is statistically significant.

\section{TABLE B7}

\section{Age by Response and Nonresponse-LPN/ LVN}

\begin{tabular}{|c|c|c|}
\hline \multicolumn{3}{|c|}{ Completed Survey } \\
\hline \multirow[t]{3}{*}{ No } & Mean & 45.8 \\
\hline & Standard deviation & 12.8 \\
\hline & $n$ & 74,736 \\
\hline \multirow[t]{3}{*}{ Yes } & Mean & 51.4 \\
\hline & Standard deviation & 13.5 \\
\hline & $n$ & 28,089 \\
\hline \multirow[t]{3}{*}{ Total } & Mean & 47.3 \\
\hline & Standard deviation & 13.2 \\
\hline & $n$ & 102,825 \\
\hline
\end{tabular}

Note. A t-test shows that this relationship is significant at the $<.001 \mathrm{lev}$ el.

\section{Weights}

In The 2015 National Workforce Survey of Registered Nurses report, nonresponse adjustments were made for gender and age. Due to the high degree of missing race/ethnicity data in the sample frame (72.0\% missing/unknown), it was still deemed impractical to use that category for nonresponse adjustment.

In order to create the combined age and gender (Age $\times$ Gender) nonresponse weights, the survey response rates for the age variable were compared at the 5-year age-group level and neighboring cells with similar response rates were collapsed. Upon completion of this process, six age-groups were created (16 to 49,50 to 54,55 to 59 , 60 to 64,65 or older, missing). These six age-groups were combined with the gender variable response categories (male, female, missing) to produce 18 Age $\times$ Gender categories. The survey response rate for each Age $\times$ Gender category (\# responding/\# in sample frame) was calculated and used to create each category's weight as follows:

Age $\times$ Gender Category Weight $=\frac{\text { Overall Survey Response Rate }}{\text { Age } \times \text { Gender Category Survey }}$
Response Rate

An example of how this was calculated can be found in the RN nonresponse sample weighting section.

Respondents in jurisdictions for which data was not obtained through the Nursys database were not weighted for age and gender and therefore all assigned an Age $\times$ Gender weight of 1.000. When the Age $\times$ Gender weights for each respondent are totaled, the sum comes to 40,272, the same as the total number of respondents. Table B8 displays the weights for the 18 Age $\times$ Gender categories in jurisdictions for which data was obtained through the Nursys database.

\section{TABLE B8}

\section{Age $\times$ Gender Response Rate-LPN/LVN}

\begin{tabular}{lccc}
\hline & \multicolumn{3}{c}{ Gender } \\
\hline Age-Group & Missing/lnvalid & Female & Male \\
\hline $18-49$ & 1.551 & 1.289 & 1.796 \\
\hline $50-54$ & 1.004 & 0.952 & 1.268 \\
\hline $55-59$ & 0.916 & 0.788 & 1.051 \\
\hline $60-64$ & 0.754 & 0.671 & 0.901 \\
\hline 65 or older & 0.643 & 0.595 & 0.733 \\
\hline Age missing & 1.097 & 1.187 & 1.647 \\
\hline
\end{tabular}

In a similar manner, poststratification weights were constructed at the state level to adjust for differing sampling rates across states. However, these adjustments were made not by comparing the number of responses in a state's sample frame count, but rather by comparing the number of responses to the number of licensees in that state. An example of how these weights were calculated can be found in the $\mathrm{RN}$ nonresponse sample weighting section.

Overall weights that combined the AgexGender and poststratification weights were created by multiplying the Age $\times$ Gender and poststratification weights for each individual to create an initial set of weights, adding the initial weights together, and slightly adjusting the weights so that they sum up to 40,272 .

The overall weights simply adjust the distribution across states, age, and gender, but sum to the actual number of LPNs/ LVNs in the subset of completed responses. They can be applied when analyzing relationships between variables without the effect of artificially altering the degrees of freedom and thereby affecting significance tests. The Age $\times$ Gender weights, poststratification weights, and overall weights are summarized in Table B9.

\section{TABLE B9}

\section{Weight Summary Statistics-LPN/LVN}

\begin{tabular}{lccccc}
\hline & $\boldsymbol{n}$ & Minimum & Maximum & Sum & Mean \\
\hline Age by gender & 40,272 & 0.595 & 1.796 & 40,272 & 1.000 \\
\hline Poststratification & 40,272 & 0.127 & 8.916 & 40,272 & 1.000 \\
\hline Combined & 40,272 & 0.074 & 8.696 & 40,272 & 1.000 \\
\hline Note. Combined weight was used in reporting results. \\
\hline
\end{tabular}




\section{List of Tables}

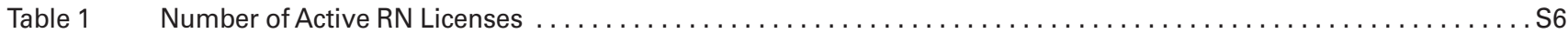

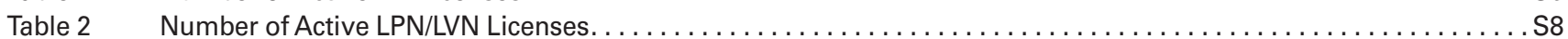

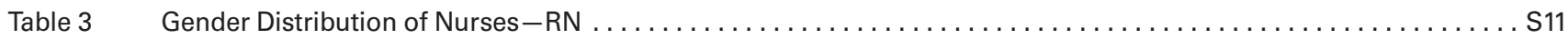

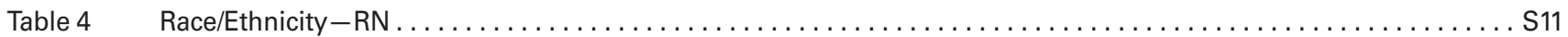

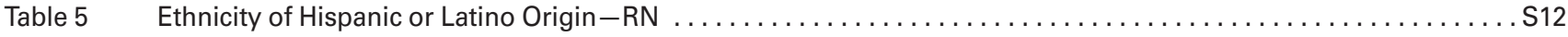

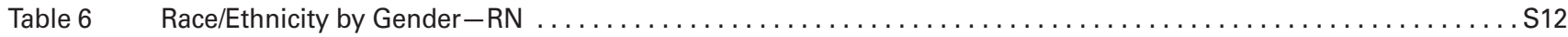

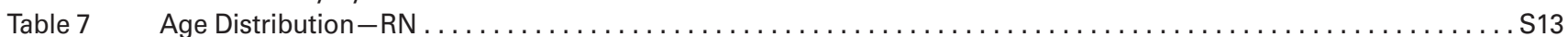

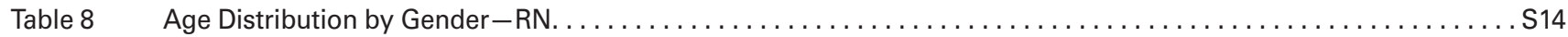

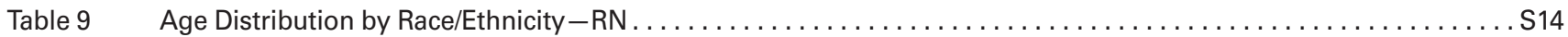

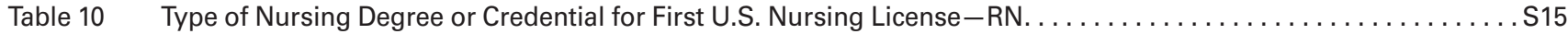

Table 11 Type of Nursing Degree or Credential for First U.S. Nursing License by Age $-\mathrm{RN} \ldots \ldots \ldots \ldots \ldots \ldots \ldots \ldots \ldots$

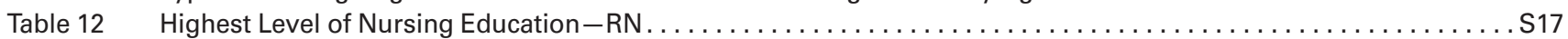

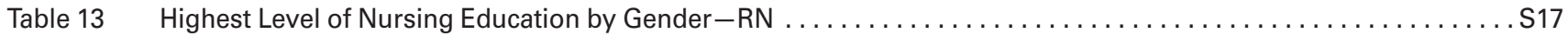

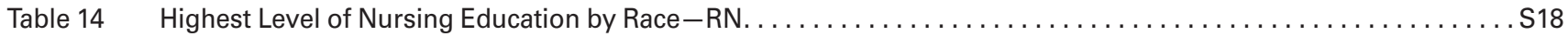

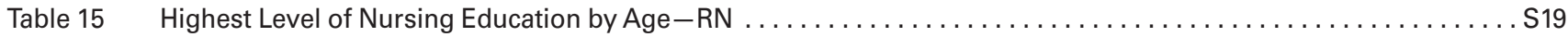

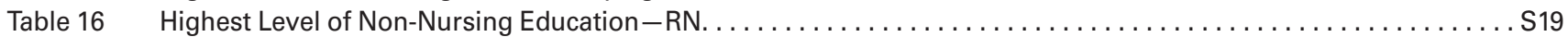

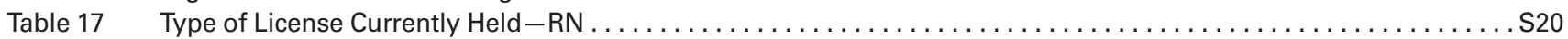

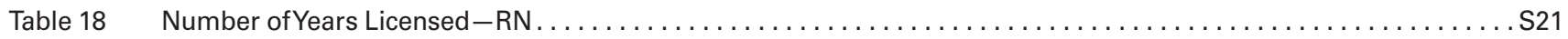

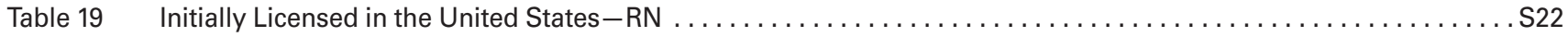

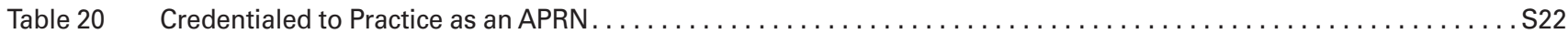

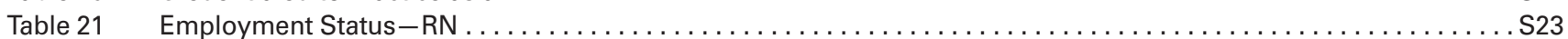

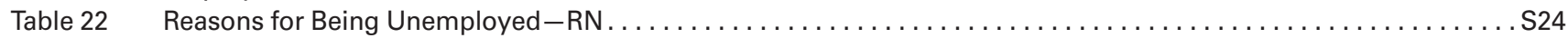

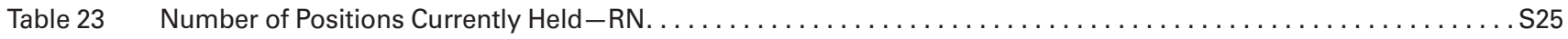

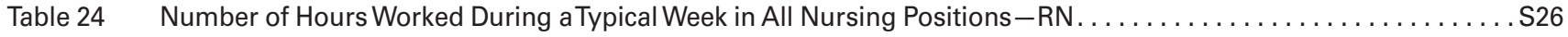

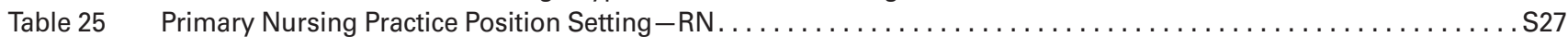

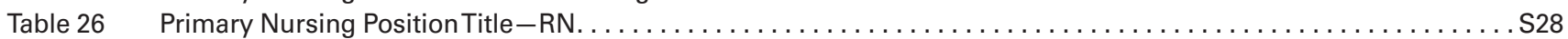

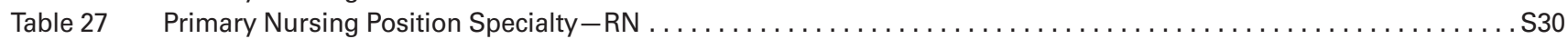

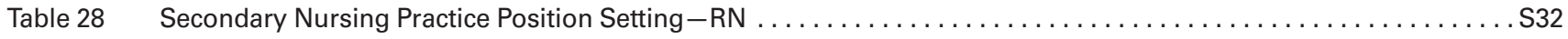

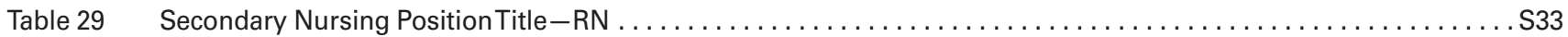

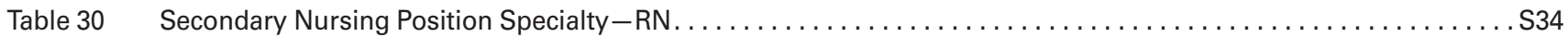

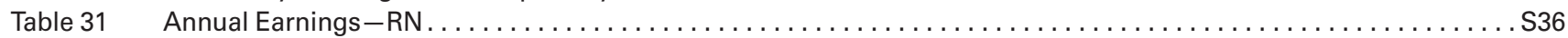

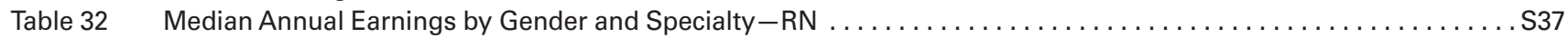

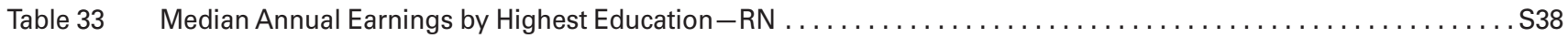

Table 34 Median Annual Earnings in Primary Nursing Position by State(s) Where Currently Practicing - RN . . . . . . . . S38

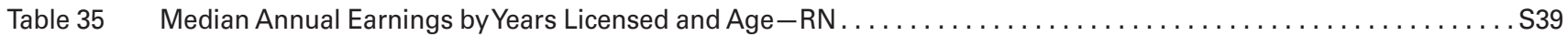

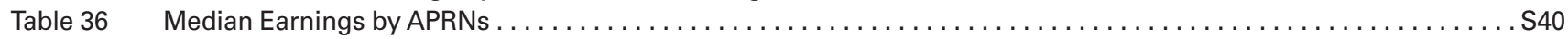

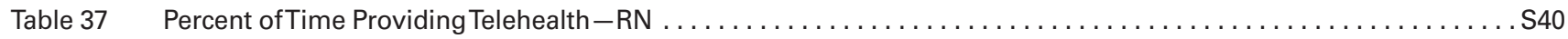

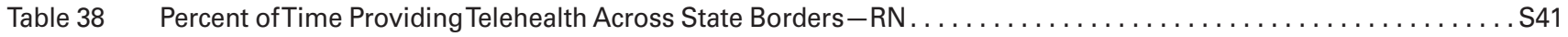

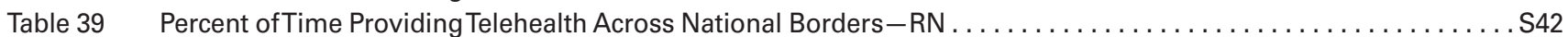

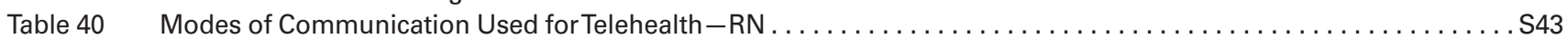

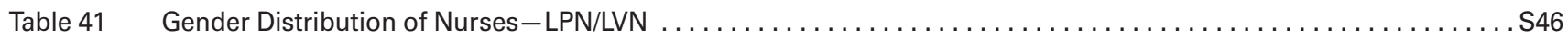

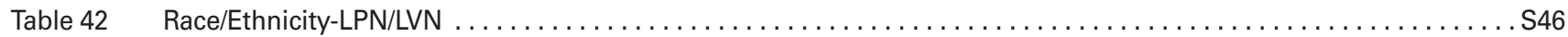

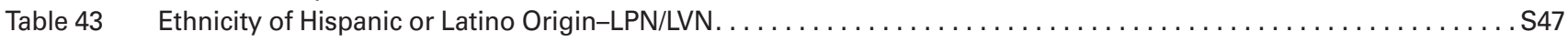

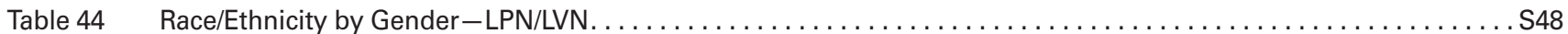

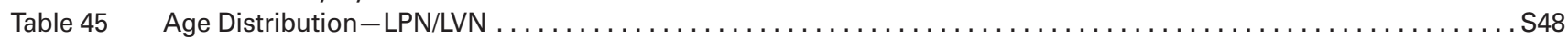

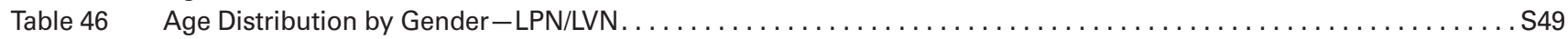

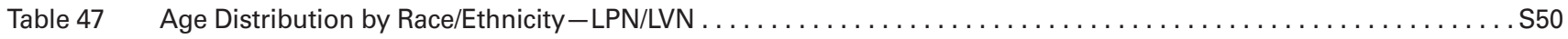

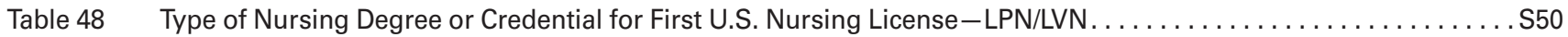

Table 49 Type of Nursing Degree or Credential for First U.S. Nursing License by Age - LPN/LVN . . . . . . . . . . . S51

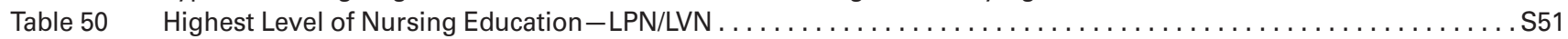

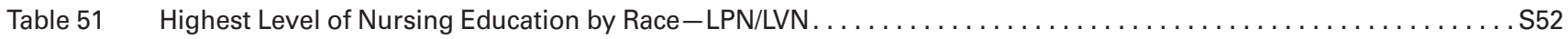

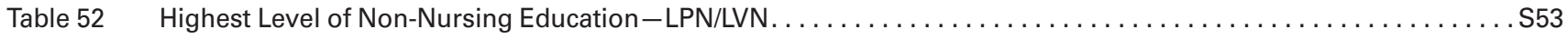

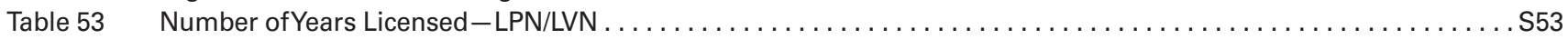

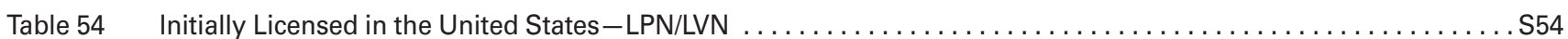

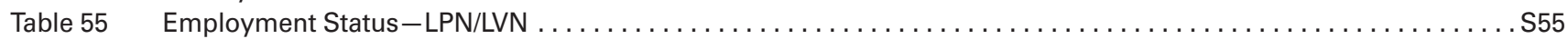

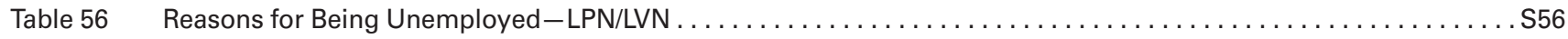

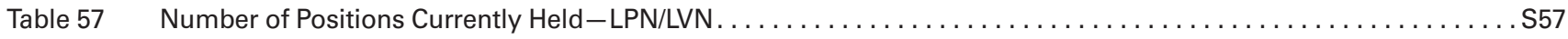

Table $58 \quad$ Number of Hours Worked During a Typical Week in All Nursing Positions - LPN/LVN $\ldots \ldots \ldots \ldots \ldots \ldots \ldots \ldots$ 
Table 59

Table 60

Table 61

Table 62

Table 63

Table 64

Table 65

Table 66

Table 67

Table 68

Table 69

Table 70

Table 71

Table 72

Table 73

Table A1

Table A2

Table A3

Table A4

Table A5

Table A6

Table A7

Table A8

Table A9

Table B1

Table B2

Table B3

Table B4

Table B5

Table B6

Table B7

Table B8

Table B9

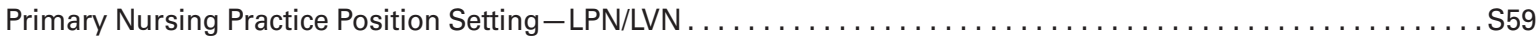

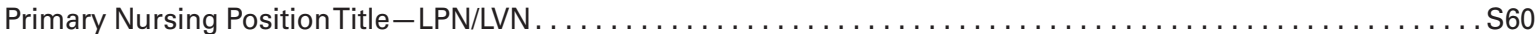

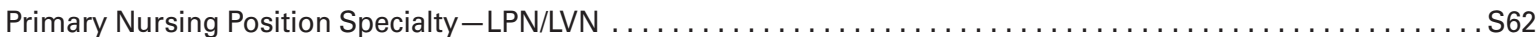

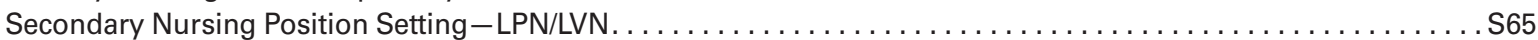

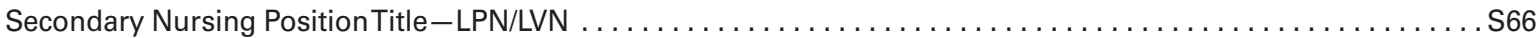

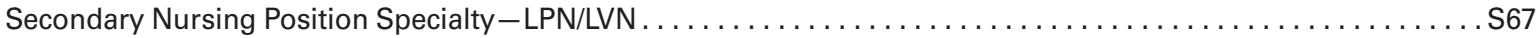

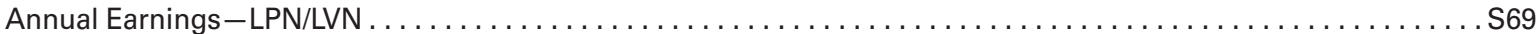

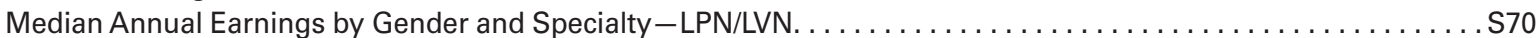

Median Annual Earnings by Highest Education-LPN/LVN $\ldots \ldots \ldots \ldots \ldots \ldots \ldots \ldots \ldots \ldots \ldots \ldots \ldots \ldots \ldots \ldots$ S71

Median Annual Earnings in Primary Nursing Position by State(s) Where Currently Practicing - LPN/LVN . . . . . . . S71

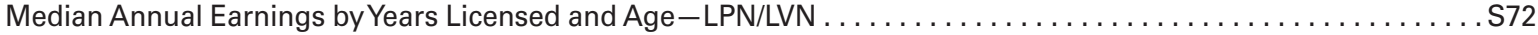

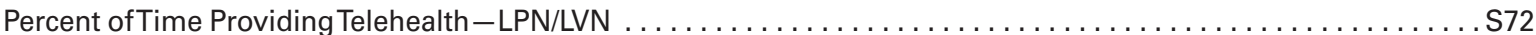

Percent ofTime Providing Telehealth Across State Borders - LPN/LVN $\ldots \ldots \ldots \ldots \ldots \ldots \ldots \ldots \ldots \ldots \ldots \ldots$. $\ldots \ldots$

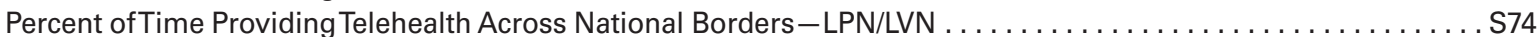

Modes of Communication Used forTelehealth - LPN/LVN $\ldots \ldots \ldots \ldots \ldots \ldots \ldots \ldots \ldots \ldots \ldots \ldots \ldots \ldots$

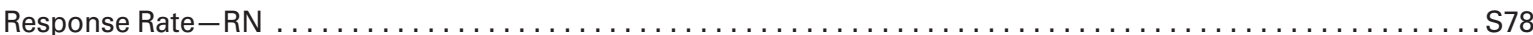

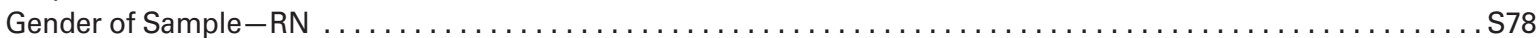

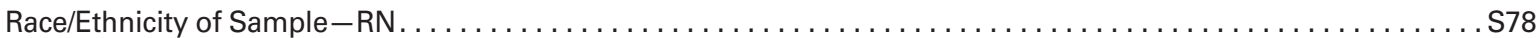

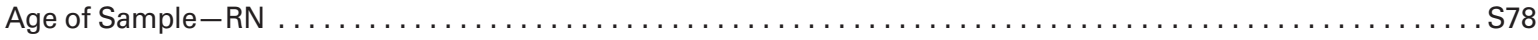

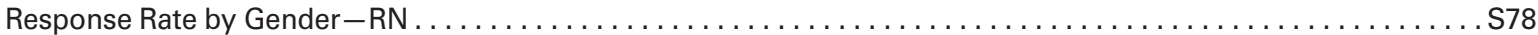

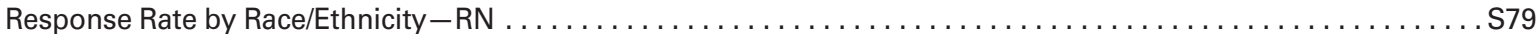

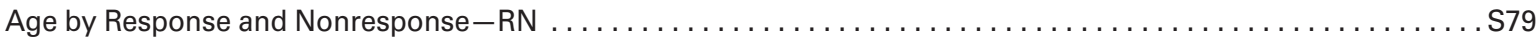

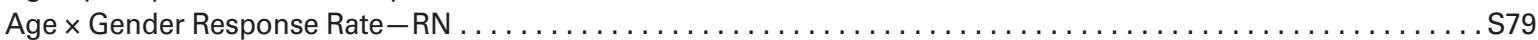

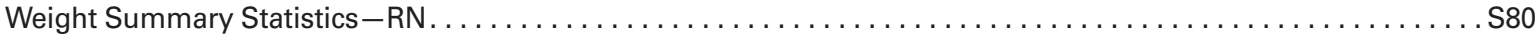

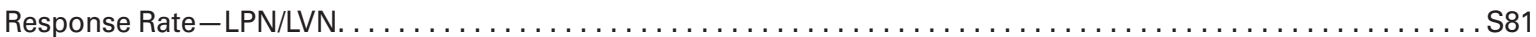

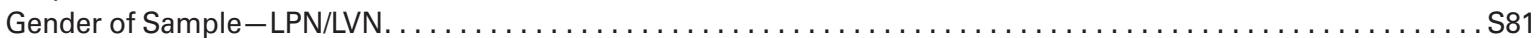

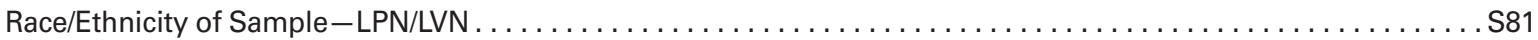

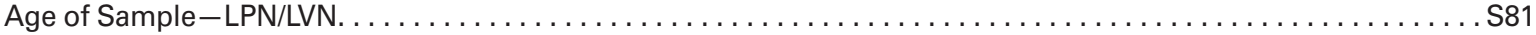

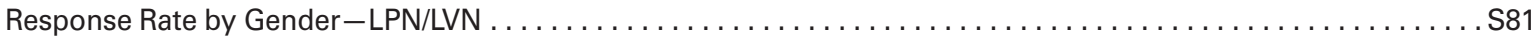

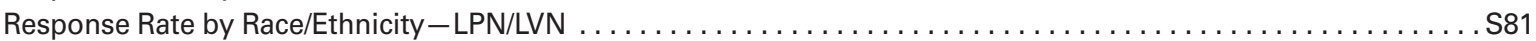

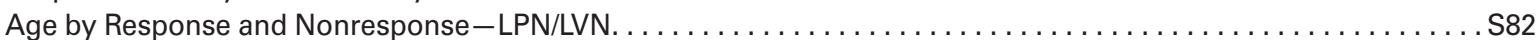

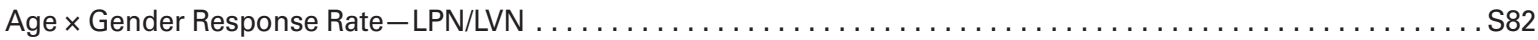

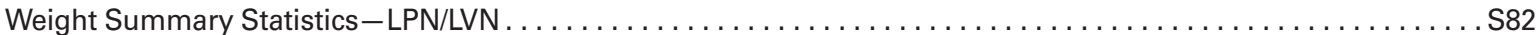




\section{National Workforce Study Questionnaire}

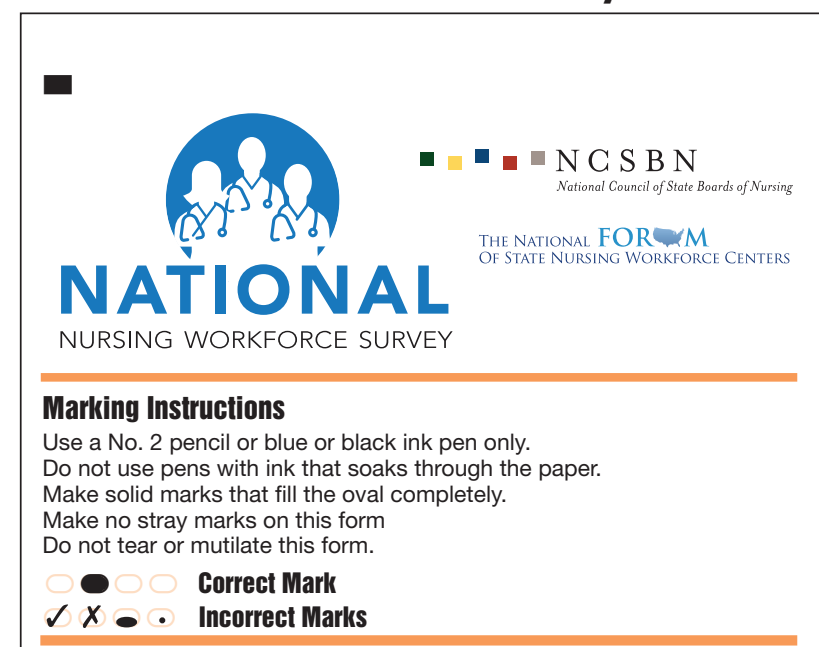

Unless indicated, select one answer per question.

\section{DEMOCT:}

1. What is your gender?

$$
\begin{aligned}
& \bigcirc \text { Male } \\
& \text { Female }
\end{aligned}
$$

2. Are you of Hispanic or Latino origin?
$\bigcirc$ Yes
$\bigcirc$ No

3. What is your race? (Select all that apply)
$\checkmark$ American Indian or Alaska Native
$\bigcirc$ Asian
Black/African American
$\checkmark$ Native Hawaiian or Other Pacific Islander
$\bigcirc$ White/Caucasian
$\bigcirc$ Other

4. In what year were you born?

\section{YEAR}

5. What type of nursing degree/credential qualified you for your first U.S. nursing license?

$\bigcirc$ Vocational/Practical certificate-nursing

Diploma-nursing

$\bigcirc$ Associate degree-nursing

Baccalaureate degree-nursing

Master's degree-nursing

$\bigcirc$ Doctoral degree-nursing $(\mathrm{PhD})$

$\bigcirc$ Doctoral degree-nursing (DNP)
6. What is your highest level of nursing education?

$\checkmark$ Vocational/Practical certificate-nursing

Diploma-nursing

Associate degree-nursing

Baccalaureate degree-nursing

Master's degree-nursing

$\bigcirc$ Doctoral degree-nursing (PhD)

Doctoral degree-nursing practice (DNP)

Doctoral degree-nursing other

7. What is your highest level of non-nursing education?

Associate degree-non-nursing

$\bigcirc$ Baccalaureate degree-non-nursing

$\bigcirc$ Master's degree-non-nursing

$\bigcirc$ Doctoral degree-non-nursing

Not applicable

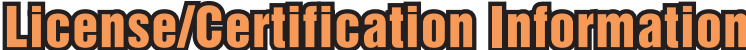

8. What type of license do you currently hold? (Select all that apply)

$\bigcirc \mathrm{RN}$

$\bigcirc$ LPN

$\bigcirc$ APRN

9. Year of Initial U.S. Licensure:

\section{YEAR}

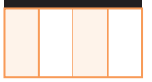

10. Were you initially licensed as an RN or LPN in the United States?

$\bigcirc$ Yes
No

11. If no, in what country were you initially licensed/ registered as an RN or LPN?

12. What is the status of the license currently held? $\bigcirc$ Active

$\bigcirc$ Inactive

13. Indicate whether you are credentialed in your state to practice as any of the following: (Select all that apply)

$\bigcirc$ Certified Nurse Practitioner

$\bigcirc$ Clinical Nurse Specialist

$\bigcirc$ Certified Registered Nurse Anesthetist

Certified Nurse Midwife

$\checkmark$ Not credentialed as any of the above 


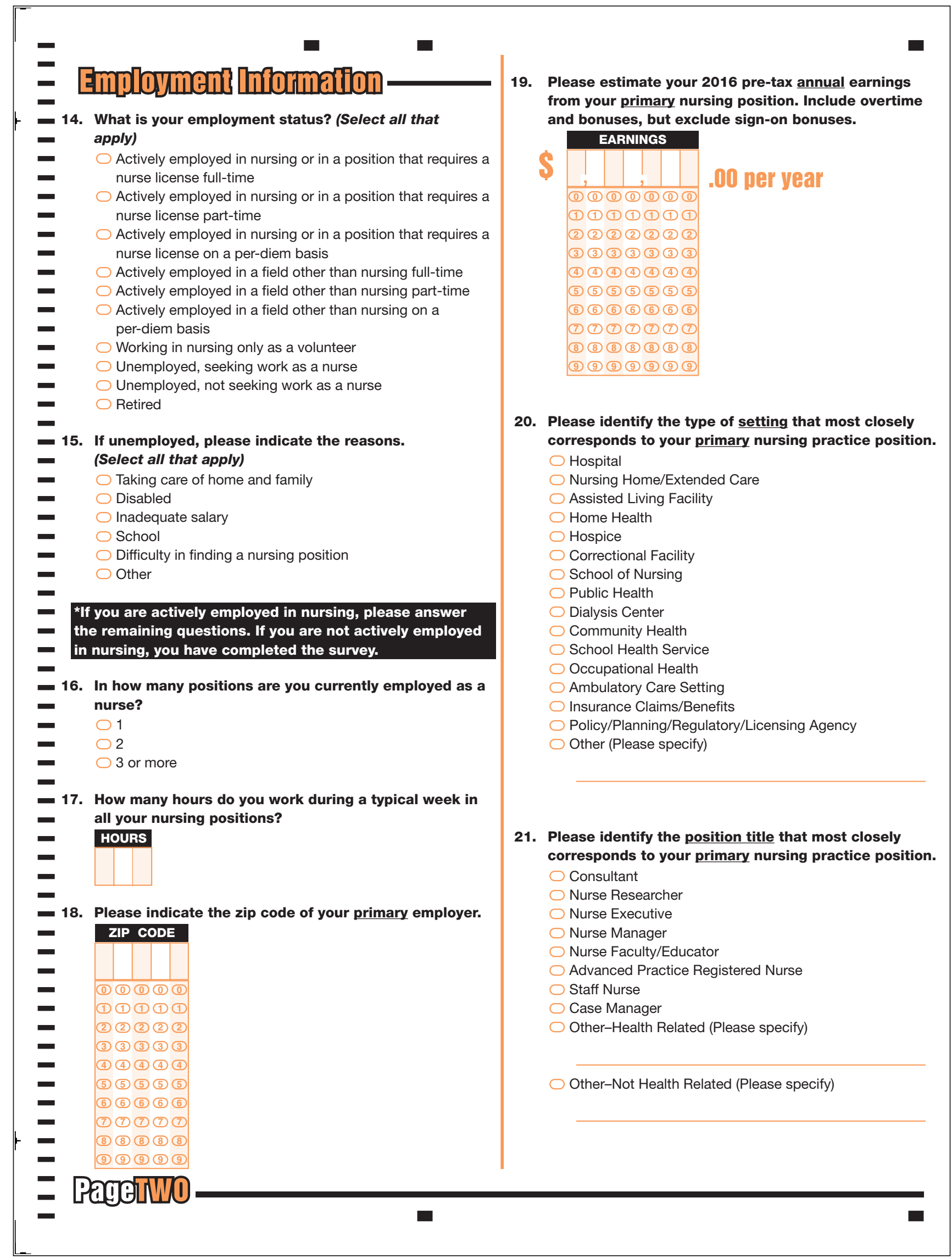


22. Please identify the employment specialty that most closely corresponds to your primary nursing practice position.
Acute Care/Critical Care
$\checkmark$ Adult Health
Family Health
Anesthesia
Cardiology
$\bigcirc$ Community
$\checkmark$ Geriatric/Gerontology
Home Health
Maternal-Child Health/Obstetrics
Medical Surgical
Nephrology
Occupational Health
Oncology
Palliative Care/Hospice
Pediatrics
Neonatal
Perioperative
Public Health
$\bigcirc$ Psychiatric/Mental Health/Substance Abuse
Rehabilitation
$\bigcirc$ School Health
$\bigcirc$ Emergency/Trauma
Women's Health
Other-Clinical specialties (Please specify)

Other-Non-clinical specialties (Please specify)

23. Please identify the type of setting that most closely corresponds to your secondary nursing practice position.

No Secondary Practice Position

Hospital

Nursing Home/Extended Care

$\bigcirc$ Assisted Living Facility

Home Health

Hospice

Correctional Facility

School of Nursing

Public Health

Dialysis Center

$\checkmark$ Community Health

$\checkmark$ School Health Service

Occupational Health

Ambulatory Care Setting

$\checkmark$ Insurance Claims/Benefits

Policy/Planning/Regulatory/Licensing Agency

$\bigcirc$ Other (Please specify)
24. Please identify the position title that most closely corresponds to your secondary nursing practice position.

No Secondary Practice Position

$\bigcirc$ Consultant

Nurse Researcher

Nurse Executive

Nurse Manager

$\bigcirc$ Nurse Faculty/Educator

Advanced Practice Registered Nurse

Staff Nurse

Case Manager

$\bigcirc$ Other-Health Related (Please specify)

Other-Not Health Related (Please specify)

25. Please identify the employment specialty that most closely corresponds to your secondary nursing practice position.

No Secondary Practice Position

Acute Care/Critical Care

Adult Health

$\checkmark$ Family Health

Anesthesia

Cardiology

Community

Geriatric/Gerontology

Home Health

Maternal-Child Health/Obstetrics

Medical Surgical

Nephrology

$\bigcirc$ Occupational Health

$\bigcirc$ Oncology

$\bigcirc$ Palliative Care/Hospice

$\checkmark$ Pediatrics

$\bigcirc$ Neonatal

$\checkmark$ Perioperative

$\checkmark$ Public Health

$\checkmark$ Psychiatric/Mental Health/Substance Abuse

Rehabilitation

School Health

$\bigcirc$ Emergency/Trauma

$\bigcirc$ Women's Health

$\bigcirc$ Other-Clinical specialties (Please specify)

Other-Non-clinical specialties (Please specify) 


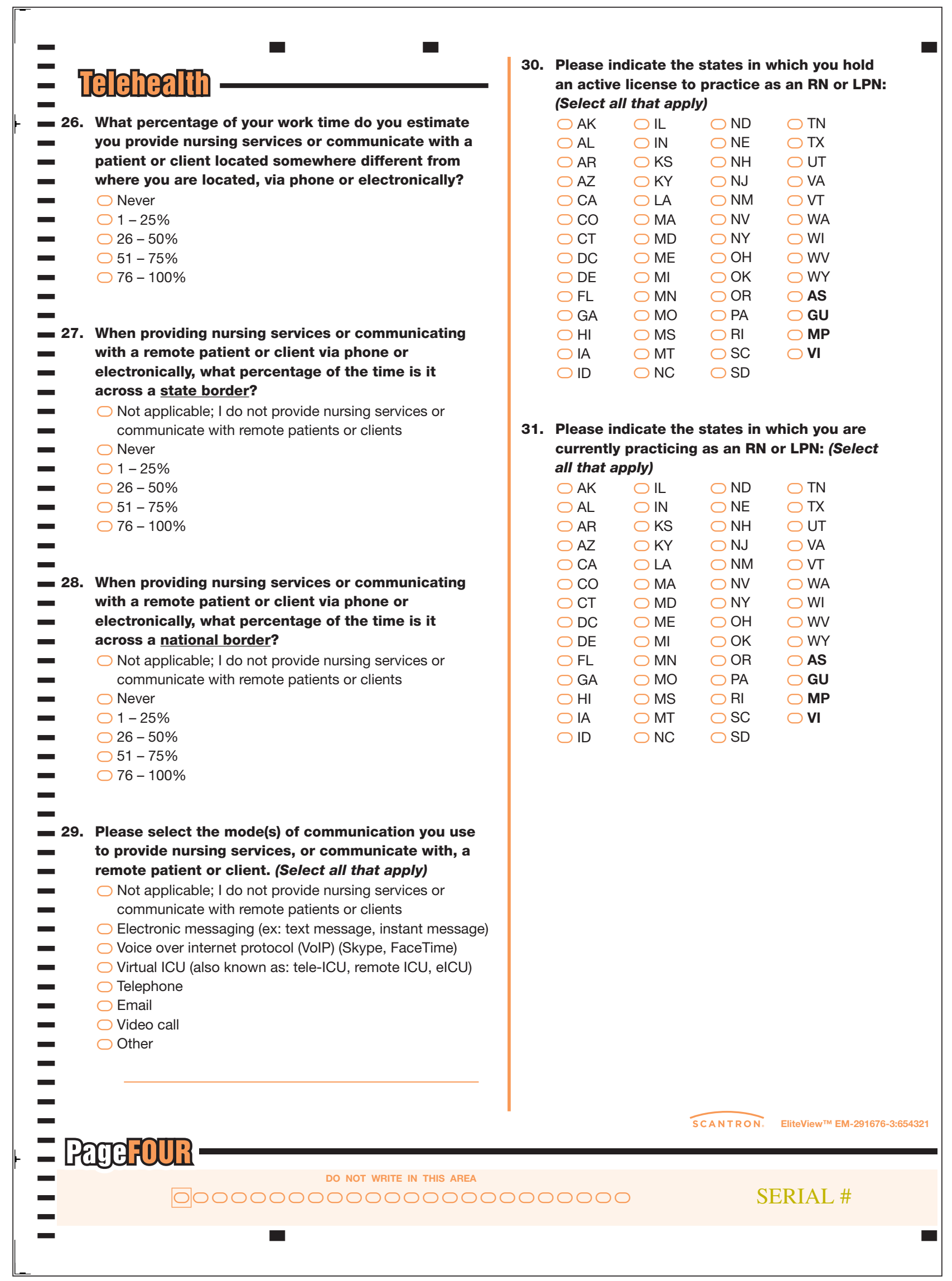

